\title{
Status Report: \\ Pretreatment Chemistry \\ Evaluation-Wash and Leach \\ Factors for the Single-shell Tank Waste Inventory
}

N. G. Colton

September 1996

Prepared for the U.S. Department of Energy under Contract DE-AC06-76RLO 1830

Pacific Northwest National Laboratory Operated for the U.S. Department of Energy by Battelle 


\title{
DISCLAIMER
}

This report was prepared as an account of work sponsored by an agency of the United States Government. Neither the United States Government nor any agency thereof, nor Battelle Memorial Institute, nor any of their employees, makes any warranty, express or implied, or assumes any legal liability or responsibility for the accuracy, completeness, or usefulness of any information, apparatus, product, or process disclosed, or represents that its use would not infringe privately owned rights. Reference herein to any specific commercial product, process, or service by trade name, trademark, manufacturer, or otherwise does not necessarily constitute or impiy its endorsement, recommendation, or favoring by the United States Government or any agency thereof, or Battelle Memorial Institute. The views and opinions of authors expressed herein do not necessarily state or reflect those of the United States Government or any agency thereof.

\author{
PACIFIC NORTHWEST NATIONAL LABORATORY \\ operated by \\ BATTEL.LE \\ for the \\ UNITED STATES DEPARTMENT OF ENERGY \\ under Coniract DE-ACO6-76RLO 1830
}

\section{Printed in the United States of Amerion}

Available to DOE and DOE contractors from the Orice of Scientific and Technical Information, P.0. Bax 62, Oak Ridge, TN 37831; prices available from (615) 576-8401.

\footnotetext{
Available to the public from the National Technical Information Service, U.S. Department of Commerce, 5285 Port Royal Rd., Springfield, VA 22161
} 
PNNL-11290

Status Report:

Pretreatment Chemistry

Evaluation-Wash and Leach

Factors for the Single-shell Tank

Waste Inventory

N. G. Colton

September 1996

Prepared for

the U.S. Department of Energy

under Contract DE-AC06-76RLO 1830

Pacific Northwest National Laboratory

Richland, Washington 99352 


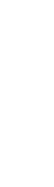




\section{Summary}

This report discusses a weighting methodology developed to depict overall wash and leach factors for the Hanford single-shell tank (SST) inventory. The factors derived from this methodology, which is based on available partitioning data, are applicable to a composite SST inventory rather than only an assumed insoluble portion. The purpose of considering the entire inventory is to provide a more representative picture of the partitioning behavior of the analytes during envisioned waste retrieval and processing activities.

The work described in this report was conducted by the Pretreatment Chemistry Evaluation task of the Tank Waste Remediation System (TWRS). Wash and leach factors, developed as part of this task, will be incorporated into the TWRS Process Technical Baseline. The wash factors will be used to partition the SST inventory into soluble and insoluble portions. The leach factors will be used to estimate the further removal of analytes, such as sodium, aluminum, phosphate, and other minor components.

Wash and leach factors are given here for 18 analytes, including chromium, phosphate, and aluminum, elements expected to drive the volume of material disposed of as high-level waste (HLW). Results from detailed evaluations using the weighting methodology are given in the form of worksheets that show the 1) distribution of a particular analyte (in metric tons) among wash and leach steps and in the residual solids and 2) analyte-specific wash factors (\% of analyte removed from initial solids) and leach factors (\% of analyte removed from washed solids).

Results from this work indicate that with the exception of chromium, analyte partitioning assumptions used in the past for planning purposes tend to be supported by the newly evaluated wash and leach factors. As a result of the newly derived chromium leach factor, there is a potential twofold increase in chromium in solids destined for disposal in HLW glass. In turn, there is a concern that the total chromium inventory may be larger than originally estimated. 



\section{Contents}

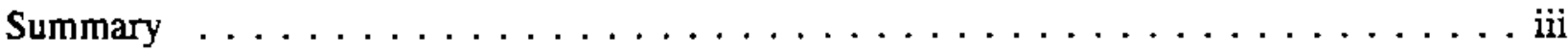

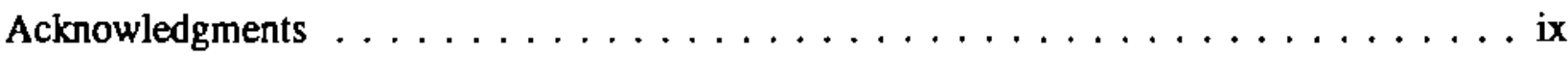

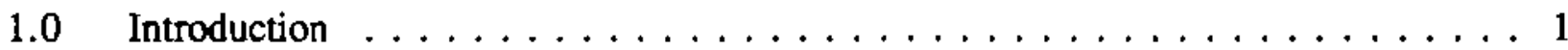

$2.0 \quad$ Methodology $\ldots \ldots \ldots \ldots \ldots \ldots \ldots \ldots \ldots \ldots \ldots \ldots \ldots \ldots \ldots$

2.1 Data Compilation and Review $\ldots \ldots \ldots \ldots \ldots \ldots$

2.2 Wash and Leach Factor Evaluation $\ldots \ldots \ldots \ldots \ldots \ldots$

3.0 Summary of Results $\ldots \ldots \ldots \ldots \ldots \ldots \ldots \ldots \ldots \ldots \ldots$

Analyte Wash and Leach Factor Evaluations

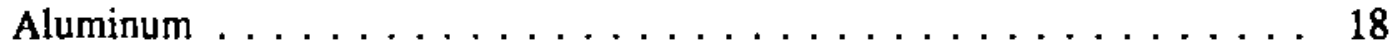

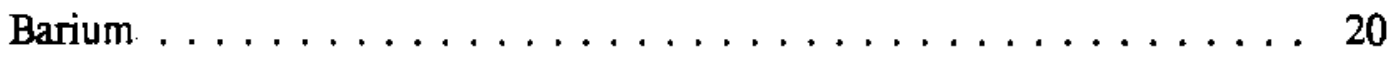

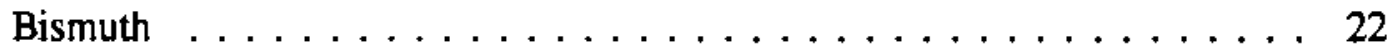

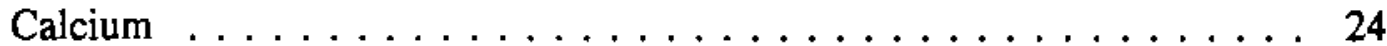

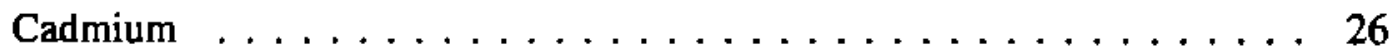

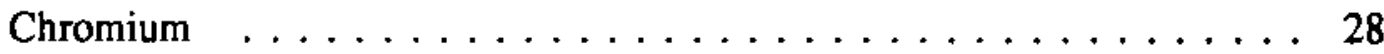

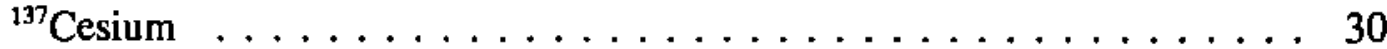

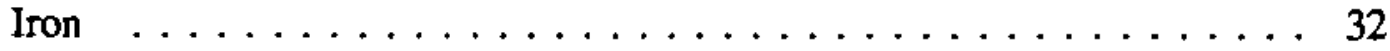

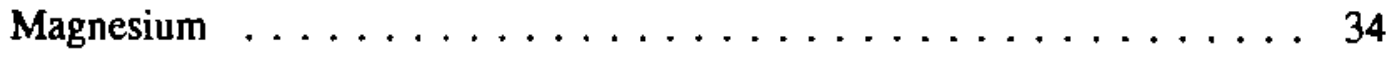

Manganese . . . . . . . . . . . . . . . . 36

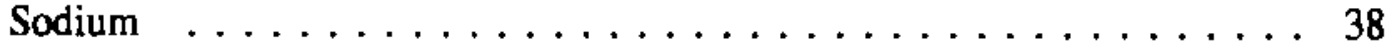

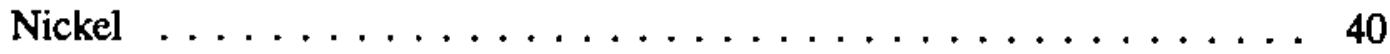

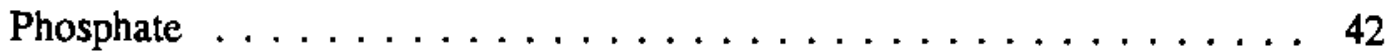

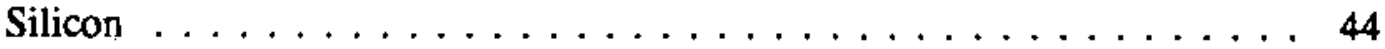

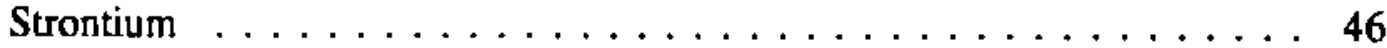

${ }^{90}$ Strontium $\ldots \ldots \ldots \ldots \ldots \ldots \ldots \ldots \ldots \ldots \ldots$

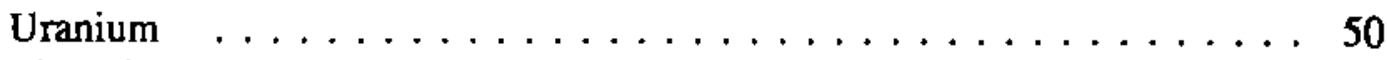

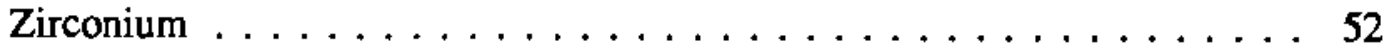

4.0 Conclusions and Recommendations $\ldots \ldots \ldots \ldots \ldots \ldots \ldots$

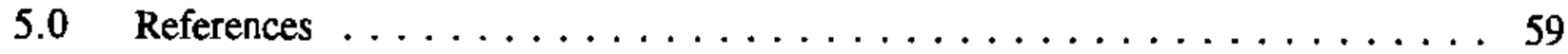




\section{Appendices}

Appendix A: Tank Waste Data Summary Worksheets

\begin{tabular}{|c|c|}
\hline-104 & \\
\hline 3-105 & $\ldots \ldots \ldots \ldots \ldots \ldots \ldots \ldots$ \\
\hline 32 & 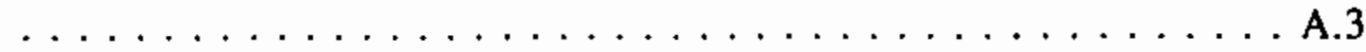 \\
\hline 109 & \\
\hline 04 & 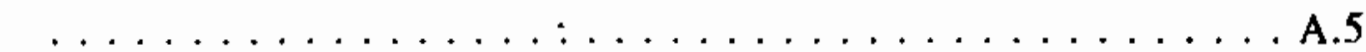 \\
\hline BY-110 & $\ldots \ldots \ldots \ldots \ldots \ldots \ldots \ldots \ldots \ldots \ldots$ \\
\hline $\mathrm{C}-107$ & 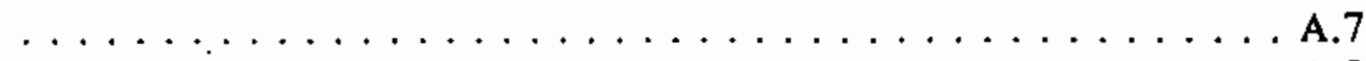 \\
\hline-108 & $\ldots \ldots \ldots \ldots \ldots \ldots \ldots$ \\
\hline-105 & $\ldots \ldots$ \\
\hline S-107 & $\ldots \ldots \ldots \ldots$ A. \\
\hline-109 & $\ldots \ldots \ldots \ldots \ldots \ldots \ldots$ A. \\
\hline 110 & $\ldots \ldots$ A. \\
\hline 102 & \\
\hline 03 & \\
\hline$T-105$ & \\
\hline 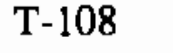 & ... \\
\hline & $\cdots \cdot \cdot$ \\
\hline & \\
\hline
\end{tabular}

Appendix B: Experimental Washing and Leaching Data Summary Worksheets

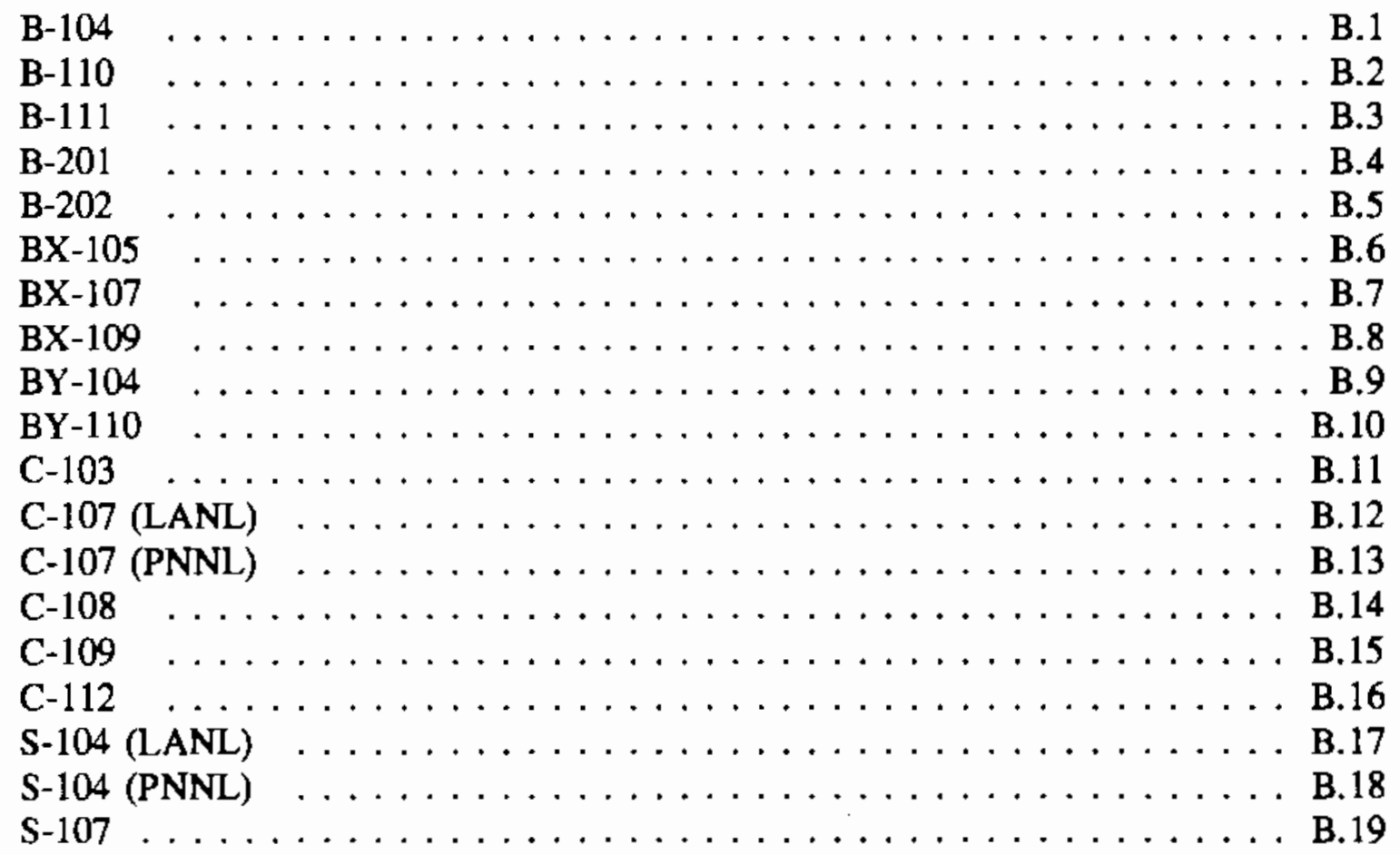




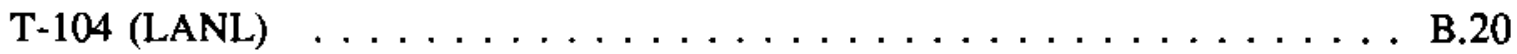

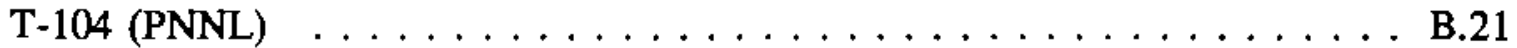

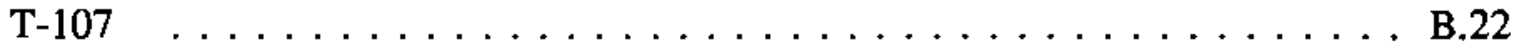

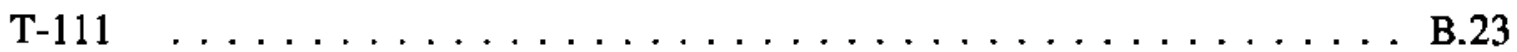

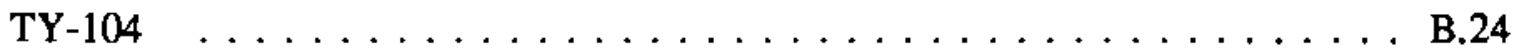

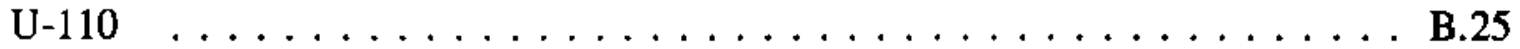

Figure

Figure 1. Pictorial Summary of Relationships Among Chemical Processes/

Campaigns, Waste Streams, Characteristic Waste-type Groups, and Single-shell Tanks $\ldots \ldots \ldots \ldots \ldots \ldots \ldots$

\section{Tables}

Table 1. Summary of Single-shell Tank Wastes Used in Washing and

Leaching Tests . . . . . . . . . . . . . . . . 2

Table 2. Reviewed Data Sources for 42 Single-shell Tanks $\ldots \ldots \ldots \ldots \ldots$

Table 3. Summary of Wash and Leach Factors for the Single-shell Tank

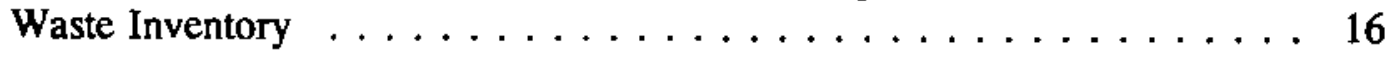

Table 4. Comparison of Total Residual Solids for SST Analytes Based on 1995 and Newly Derived Partitioning Factors . . . . . . . . . 57 


\section{"}




\section{Acknowledgments}

The author wishes to thank the following persons who provided valuable assistance and input to this task: D. J. Washenfelder, A. L. Boldt, and M. J. Kupfer (Westinghouse Hanford Company); G. J. Lumetta, B. M. Rapko, and S. R. Gano (Pacific Northwest National Laboratory); D. J. Temer (Los Alamos National Laboratory); and G. L. Borsheim (Borsheim Associates). 


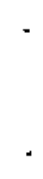




\subsection{Introduction}

After the primary mission at the Hanford Site changed from plutonium production to environmental restoration (1990), the Tank Waste Remediation System (TWRS) was established by the U.S. Department of Energy (DOE) to safely manage and dispose of highlevel wastes (HLW) stored in the underground tanks. A key element of the disposal activities under TWRS involves establishing a reference strategy for pretreating wastes prior to disposal. Pretreatment strategies include utilizing processes and equipment to separate and/or destroy waste components to minimize treated waste volumes and to produce waste fractions that are compatible with final waste forms and their disposal criteria.

The currently envisioned pretreatment strategy proposes 1) to remove radionuclides from the aqueous waste fractions to produce streams suitable for disposal as low-level waste (LLW) and 2) to use washing and selective leaching strategies to remove elements from sludges expected to drive HLW production, i.e., aluminum, phosphorus, and chromium. The effect of this pretreatment strategy on final waste volumes is evaluated in systems engineering studies, such as the TWRS Process Flowsheet (Orme 1995), by employing assumptions about the chemical and physical behavior of the wastes. Included in these assumptions are wash and leach factors, i.e., the fraction of each waste component expected to dissolve or to leach from waste streams during various processing steps.

In the past, the single-shell tank (SST) and double-shell tank (DST) waste inventories were partitioned into soluble (salt) and insoluble (sludge) portions based on the judgment of experienced personnel. Estimated sludge wash factors were employed to further separate residual soluble material from the insoluble sludge portion. These estimated sludge wash factors (Boomer et al. 1993) were derived from a very limited set of available data. Portions of this set were updated in 1994 by the TWRS Pretreatment Chemistry task by analyzing results from waste characterization studies of 27 SST sludges (Colton 1995).

Since 1994, as part of the TWRS Program to provide experimental data and a technical basis for wash and leach factors, washing and caustic leaching tests of Hanford tank sludges have been performed by Pacific Northwest National Laboratory (PNNL) ${ }^{(\infty)}$ and Los Alamos National Laboratory (LANL). Tests have been completed on $23^{(\text {b) }}$ individual SST

(a) Operated for the U.S. Department of Energy by Battelle under Contract DEAC06-76RLO 1830.

(b) While washing and leaching tests have been completed for SST SX-108, results from these tests were not available for consideration in this evaluation. 
wastes and 1 DST waste. The bulk of the total waste solids inventory (sludges, salt cake, and associated interstitial liquid) is in the SSTs. Table 1 summarizes the 23 SST wastes in terms of process origin, number of wastes tested from each process, and representative tank waste volumes and volume fractions for each process.

Table 1. Summary of Single-shell Tank Wastes Used in Washing and Leaching Tests

\begin{tabular}{||l|c|c|c||}
\hline Process Origin & $\begin{array}{c}\text { Number of } \\
\text { Tank Wastes }\end{array}$ & $\begin{array}{c}\text { Representative } \\
\text { Volume (kL) }\end{array}$ & $\begin{array}{c}\text { Volume } \\
\text { Fraction (\%) }\end{array}$ \\
\hline Bismuth Phosphate & 11 & 10200 & 55.8 \\
\hline Uranium Recovery & 6 & 1930 & 10.6 \\
\hline Redox & $3^{(a)}$ & 2860 & 15.6 \\
\hline Purex & 1 & 235 & 1.29 \\
\hline $\begin{array}{l}\text { Evaporator } \\
\text { Campaign } / \mathrm{s}^{(0)}\end{array}$ & 2 & 3050 & 16.7 \\
\hline Totals & 23 & 18275 & 100 \\
\hline
\end{tabular}

(a) Results from tests performed with one of these wastes, $\mathrm{SX}-108$, were not available for consideration in this evaluation.

(b) Predominantly salt-type wastes.

This table shows that 17 of the washing and leaching tests were performed with wastes that originated from the Bismuth Phosphate and Uranium Recovery processes. As a result, overall wash and leach factors derived solely by weighting individual test results on a tank volume basis could be unduly influenced by partitioning behaviors observed in these 17 wastes. Therefore, a modified weighting scheme was developed to estimate overall wash and leach factors that would be more representative of the entire SST solids inventory.

This report presents the modified weighting methodology, developed by the TWRS Pretreatment Chemistry Evaluation task, and the overall partitioning factors derived from it. The methodology is described in Section 2.0. The overall wash and leach factor results are given in Section 3.0 in the form of detailed evaluations by analyte, and conclusions and recommendations are offered in Section 4.0. Supporting worksheets, i.e., individual tank data summary worksheets and tank-specific, experimental washing and leaching data summary worksheets, are contained in Appendices A and B, respectively. 


\subsection{Methodology}

In FY 1996, the Pretreatment Chemistry Evaluation task was charged with updating currently assumed partitioning factors based on the washing and leaching results and other relevant data. An important point to be considered before developing a methodology to tie these individual results to the SST inventory was that the bulk of the chromium and aluminum inventories potentially reside in wastes from the Redox Process and evaporator campaigns. If a normalization method based solely on tank volumes were used, the four wastes tested to date from the Redox Process and evaporator campaigns would contribute to less than a third of the partitioning behaviors incorporated into the overall factors. Another point to be considered was how to incorporate data from the two predominantly salt-type wastes into factors that, in the past, had been applied only to insoluble sludges to remove residual soluble material. The modified weighting scheme was developed to estimate overall wash and leach factors, accounting for these two points, that would be more representative of the entire SST inventory. This methodology is described in further detail in the following sections.

\subsection{Data Compilation and Review}

The first step in deriving overall wash and leach factors was to compile and review relevant data generated over the years as part of characterization, washing and leaching, and radionuclide removal studies. Table 2 summarizes reviewed data sources for 42 SST wastes.

Table 2. Reviewed Data Sources for 42 Single-shell Tanks

\begin{tabular}{||c|c|c|c||}
\hline Tank & $\begin{array}{c}\text { Characterization Data } \\
\text { 1986 or later }\end{array}$ & $\begin{array}{c}\text { Sludge Washing/ } \\
\text { Leaching Data } \\
\text { 1994 or later }\end{array}$ & $\begin{array}{c}\text { Radionuclide } \\
\text { Removal Test Data } \\
\text { 1980 or earlier }\end{array}$ \\
\hline A-103 & $\begin{array}{c}\text { Colton (1995) [data from } \\
\text { Weiss and Schull (1988a)] }\end{array}$ & -- & - \\
\hline A-106 & $\begin{array}{c}\text { Colton (1995) [data from } \\
\text { Weiss and Schull (1988b)] }\end{array}$ & -- & - \\
\hline B-104 & Field et al. (1996a) & Temer and Villarreal (1996) & - \\
\hline B-105 & - & - & Schulz (1980) \\
\hline B-110 & $\begin{array}{c}\text { Colton (1995) [data from } \\
\text { PNNL letter report to WHC] }\end{array}$ & Lumetta and Rapko (1994) & - \\
\hline
\end{tabular}


Table 2. cont'd.

\begin{tabular}{|c|c|c|c|}
\hline Tank & $\begin{array}{c}\text { Characterization Data } \\
1986 \text { or Iater }\end{array}$ & $\begin{array}{l}\text { Sludge Washing/ } \\
\text { Leaching Data } \\
1994 \text { or later }\end{array}$ & $\begin{array}{c}\text { Radionuclide } \\
\text { Removal Test Data } \\
1980 \text { or earlier }\end{array}$ \\
\hline B-111 & $\begin{array}{l}\text { Colton (1995) [from } \\
\text { Giamberardini (1993)] }\end{array}$ & Rapko et al. (1995) & - \\
\hline B-201 & $\begin{array}{l}\text { Colton (1995) from [date } \\
\text { from WHC (1993a)] }\end{array}$ & Lumetta and Rapko (1994) & -- \\
\hline B-202 & $\begin{array}{c}\text { Worksheet provided in } \\
\text { Appendix } A\end{array}$ & Temer and Villarreal (1995) & - \\
\hline BX-104 & $\begin{array}{l}\text { Colton (1995) [data from } \\
\text { Weiss and Schull (1988c)] }\end{array}$ & $\cdots$ & - \\
\hline $\mathrm{BX}-105^{(\mathbf{n})}$ & $\begin{array}{l}\text { Colton (1995) [data from } \\
\text { Weiss and Schull (1988d)] }\end{array}$ & Temer and Villarreal (1995) & - \\
\hline BX-107 & Kristofski (1993) & Rapko et al. (1995) & -- \\
\hline BX-109 & Field et al. (1996b) & Temer and Villarreal (1996) & -- \\
\hline BY-104 & $\begin{array}{c}\text { Worksheet provided in } \\
\text { Appendix } A\end{array}$ & Lumetta et al. (1996) & -- \\
\hline BY-110 & $\begin{array}{c}\text { Worksheet provided in } \\
\text { Appendix A }\end{array}$ & Lumetts et al. (1996) & - \\
\hline C-103 & $\begin{array}{l}\text { Colton (1995) [data from } \\
\text { Weiss and Schull (1988e)] }\end{array}$ & Rapko et al. (1995) & - \\
\hline$C-104$ & $\begin{array}{l}\text { Colton (1995) [dats from } \\
\text { Weiss and Schull (1988f)] }\end{array}$ & -- & $\rightarrow$ \\
\hline$C-105$ & $\begin{array}{l}\text { Colton (1995) [data from } \\
\text { Weiss and Schull (1988g)] }\end{array}$ & -- & -- \\
\hline C-106 & $\begin{array}{l}\text { Colton (1995) [data from } \\
\text { Weiss and Schull (1988h)] }\end{array}$ & -- & - \\
\hline C-107 & $\begin{array}{l}\text { Colton (1995) [data from } \\
\text { Bell et al. (1995)] }\end{array}$ & $\begin{array}{l}\text { Temer and Villarreal (1995) } \\
\text { Lumetts and Rapko (1996) }\end{array}$ & $\cdots$ \\
\hline$C-108$ & $\begin{array}{l}\text { Worksheet provided in } \\
\text { Appendix A }\end{array}$ & Temer and Villarreal (1995) & -- \\
\hline C-109 & $\begin{array}{l}\text { Colton (1995) [data from } \\
\text { Simpson et al. (1993a)] }\end{array}$ & Lumetts and Rapko (1994) & -- \\
\hline$C-110$ & $\begin{array}{c}\text { Colton (1995) [data from } \\
\text { WHC (1993b)] }\end{array}$ & -- & - \\
\hline
\end{tabular}


Table 2. cont'd.

\begin{tabular}{|c|c|c|c|}
\hline Tank & $\begin{array}{c}\text { Characterization Data } \\
1986 \text { or later }\end{array}$ & $\begin{array}{l}\text { Sludge Washing/ } \\
\text { Leaching Data } \\
1994 \text { or later }\end{array}$ & $\begin{array}{l}\text { Radionuclide } \\
\text { Removal Test Data } \\
1980 \text { or earlier }\end{array}$ \\
\hline$C-112$ & $\begin{array}{l}\text { Colton (1995) [data from } \\
\text { Simpson et al. (1993b)] }\end{array}$ & Lumetta and Rapko (1994) & -- \\
\hline$S-104$ & $\begin{array}{l}\text { Colton (1995) from [data } \\
\text { from Kocher (1993)] }\end{array}$ & $\begin{array}{l}\text { Temer and Villarreal (1995) } \\
\text { Rapko et al. (1995) }\end{array}$ & -- \\
\hline$S-105$ & - & .- & Schulz (1980) \\
\hline S-107 & $\begin{array}{l}\text { Worksheet provided in } \\
\text { Appendix A }\end{array}$ & Lumetta et al. (1996) & - \\
\hline$S-109$ & - & - & Schulz (1980) \\
\hline$S-110$ & -- & -- & Schulz (1980) \\
\hline sX-102 & - & - & Schul2 (1980) \\
\hline SX-103 & - & -- & Schulz (1980) \\
\hline$T-102$ & $\begin{array}{l}\text { Colton (1995) [data from } \\
\text { WHC (1993c)] }\end{array}$ & -- & - \\
\hline$T-104$ & $\begin{array}{l}\text { Colton (1995) [data from } \\
\text { WHC (1993d)] }\end{array}$ & $\begin{array}{c}\text { Temer and Villarreal (1995) } \\
\text { Rapko et al. (1995) }\end{array}$ & -- \\
\hline $\mathrm{T}-105$ & $\begin{array}{l}\text { Worksheet provided in } \\
\text { Appendix A }\end{array}$ & -- & - \\
\hline$T \cdot 107$ & $\begin{array}{l}\text { Colton (1995) [data from } \\
\text { WHC (1993e)] }\end{array}$ & Temer and Villarreal (1995) & -- \\
\hline$T-108$ & Baldwin et al. (1996) & - & -- \\
\hline $\mathrm{T}-111$ & $\begin{array}{l}\text { Colton (1995) [data from } \\
\text { WHC (1993f)] }\end{array}$ & Rapko et al. (1995) & -- \\
\hline $\mathrm{TX}-116$ & -- & -- & Schulz (1980) \\
\hline TY-102 & $\begin{array}{c}\text { Worksheet provided in } \\
\text { Appendix A [data from } \\
\text { Weiss and Mauss (1988a)] }\end{array}$ & - & - \\
\hline
\end{tabular}


Table 2. cont'd.

\begin{tabular}{||c|c|c|c||}
\hline Tank & $\begin{array}{c}\text { Characterization Data } \\
\text { 1986 or later }\end{array}$ & $\begin{array}{c}\text { Sludge Washing/ } \\
\text { Leaching Data } \\
\text { 1994 or later }\end{array}$ & $\begin{array}{c}\text { Radionuclide } \\
\text { Removal Test Data } \\
\text { 1980 or earlier }\end{array}$ \\
\hline TY-104 & $\begin{array}{c}\text { Colton (1995) [data from } \\
\text { Weiss and Mauss (1988b)] }\end{array}$ & Temer and Villarreal (1995) & -- \\
\hline TY-105 & $\begin{array}{c}\text { Colton (1995) [data from } \\
\text { Weiss and Mauss (1988c)] }\end{array}$ & - & - \\
\hline TY-106 & $\begin{array}{c}\text { Colton (1995) [data from } \\
\text { Weiss and Mauss (1988d)] }\end{array}$ & - & - \\
\hline U-110 & $\begin{array}{c}\text { Colton (1995) [data from } \\
\text { Brown and Jenson (1993)] }\end{array}$ & Lumetta and Rapko (1994) & -- \\
\hline $\begin{array}{l}\text { (a) Data from this tank have not been included in this evaluation because of discrepancies in projected } \\
\text { inventories derived from core samples obtained in 1986 and auger surface samples obtained in 1994. (The } \\
\text { auger method generally was used to sample the first } 8 \text { inches of solids on the tank waste surface). These } \\
\text { samples may be representative of cladding waste, the last type of waste added to this tank. Washipg and } \\
\text { leaching tests were performed only on the auger surface sample. }\end{array}$ \\
\hline
\end{tabular}

Information important to deriving wash and leach factors included both analyte partitioning and inventory information. Some of this information was available from data summary worksheets that were completed for 27 SST wastes as part of an earlier activity to update sludge wash factors (Colton 1995). These worksheets provided a uniform format for reviewing data, as well as the means to verify whether the data set for each tank was complete. The analytical water wash data contained in these worksheets provided tankspecific information about the fraction of each component that dissolved with water, i.e., wash factor information. In addition, these worksheets provided analyte inventory values calculated from analyte concentrations, the waste density, and the tank waste volume.

Data summary worksheets were completed for an additional 18 SST wastes as part of this work (refer to Appendix A). Note that because some sample analyses were limited, some of these worksheets are not as detailed as others. Also note that data for 7 tanks containing predominantly salt-type wastes (B-105, TX-116, S-105, S-109, S-110, SX-102, SX-103) originated from radionuclide removal tests performed by Schulz (1980), and data for C-107 originated from washing and leaching tests performed by Temer et al. (1995). With the exception of the 7 salt-type wastes, concentrations were entered on the worksheets as reported, and no effort was made to ion balance results. In the case of the 7 salt-type wastes, sodium or nitrate was adjusted for ion balancing purposes, and the residual solids were adjusted to reflect those reported by Schulz (1980). Any adjustments to waste concentrations were noted at the bottom of each worksheet. 
As experimental washing and leaching data were an integral part of this activity, these data (Lumetta and Rapko 1994; Rapko et al. 1995; Temer and Villarreal 1995; Lumetta et al 1996; and Temer and Villarreal 1996) also were summarized on worksheets (refer to Appendix B). These worksheets projected wash and leach factors onto tank-specific inventories obtained from data summary worksheets contained in Appendix $A$ and in Colton (1995). The washing and leaching worksheets provide concentration values obtained by two different methods: 1) direct sample analysis and 2) summation of analytes distributed among the washing and leaching solutions and the residual solids. Note that there is considerable deviation among some of the analyte concentrations obtained by the two different methods. This deviation may indicate that one or the other (or possibly both) of these concentrations is not representative of the actual tank waste or that poor mass balances were obtained from summing different test results. These deviations could potentially be used to estimate error ranges for the experimentally determined wash and leach factors.

\subsection{Wash and Leach Factor Spreadsheet Evaluation}

The second step in deriving overall wash and leach factors entailed entering individual tank waste data into a spreadsheet, in terms of metric tons of 1) analyte inventory, 2) analyte removed with washing, and 3) analyte removed with alkaline leaching. In this spreadsheet, the analyte amounts were then weighted according to the volume contribution of their characteristic waste type. Characteristic waste type groups were assigned by using the Sort on Radioactive Waste Type (SORWT) model (Hill et al. 1995). This model categorizes tanks into groups expected to have similar physical and chemical compositions.

The relationship among individual tank wastes and characteristic waste types, according to the SORWT model, is depicted schematically in Figure 1. Tank-specific wastes that were included in this evaluation are marked in the figure as follows: wastes with washing and leaching test data are represented by dark shading; wastes with only wash information, derived from water leach tests obtained during characterization studies, are represented by light shading; and salt-type wastes with dissolution data obtained from radionuclide removal tests are represented by a double box. Note in Figure 1 that not all characteristic waste-type groups have corresponding tanks with available waste data.

For this evaluation, the following assumptions were made:

- Redox sludges were removed from Redox/Evaporator Bottom (SORWT Group I), Redox (SORWT Group IV), Evaporator Bottom/Redox (SORWT Group IX), and Evaporator Bottom/Cladding Waste (SORWT Group VI) characteristic waste groups and repartitioned as follows: 
- $\quad$ Redox boiling sludges $(4290 \mathrm{~kL}): \mathrm{S}-104, \mathrm{SX}-107, \mathrm{SX}-108, \mathrm{SX}-109, \mathrm{SX}-111$, SX-112, SX-114.

- $\quad$ Redox nonboiling sludges (4750 kL): SX-110, SX-115, U-101 and $5440 \mathrm{~kL}$ sludge from Redox/Evaporator Bottom (SORWT Group I) (including S-107), $481 \mathrm{~kL}$ sludge from Evaporator Bottom/Redox (SORWT Group IX), and 1190 $\mathrm{kL}$ sludge from Evaporator Bottom/Cladding Waste (SORWT Group VI). Sludge volumes for individual tanks are based on Hanlon (1995).

- Characteristic waste-type groups with no available individual tank waste data were assigned representative tanks that are believed to have similar waste compositions, e.g., first-cycle bismuth phosphate waste (1C) and first-cycle bismuth phosphate waste with additional salts added to the waste from evaporator campaigns (1C/EB).

- Mass of analyte in a particular characteristic waste type $\mathbf{M}_{\mathrm{A(c)}}$ is given by:

$$
\mathbf{M}_{\mathrm{A}(\mathrm{c})}=\sum \mathrm{m}_{\mathrm{A}(\mathrm{i})}\left(\frac{\mathrm{V}_{\mathrm{c}}}{\sum \mathrm{V}_{\mathrm{i}}}\right)
$$

where

$$
\begin{array}{ll}
\mathbf{M}_{\mathbf{A}(\boldsymbol{c})} & =\text { mass of analyte in a particular waste type } \\
\mathbf{m}_{\boldsymbol{A}(i)} & =\text { mass of analyte in an individual waste } \\
\mathbf{V}_{\mathrm{c}} & =\text { volume of characteristic waste } \\
\mathrm{V}_{\mathrm{i}} & =\text { volume of individual waste }
\end{array}
$$

Tank volumes for wastes that contribute only wash data are not included in the summation of volumes used to weight leach data.

- For waste types originating from the Purex Process, where wash information derived from characterization analyses were available, metric tons of analyte leached were calculated using leach factors from a waste type expected to exhibit similar leaching behavior. Specific assumptions are noted at the end of each analyte worksheet.

- For wastes that were tested by both PNNL and LANL (C-107, S-104, and T-104), metric tons of washed and leached analyte were calculated by averaging the results from each analysis. In some cases, results for a minor analyte were only reported by one laboratory. 


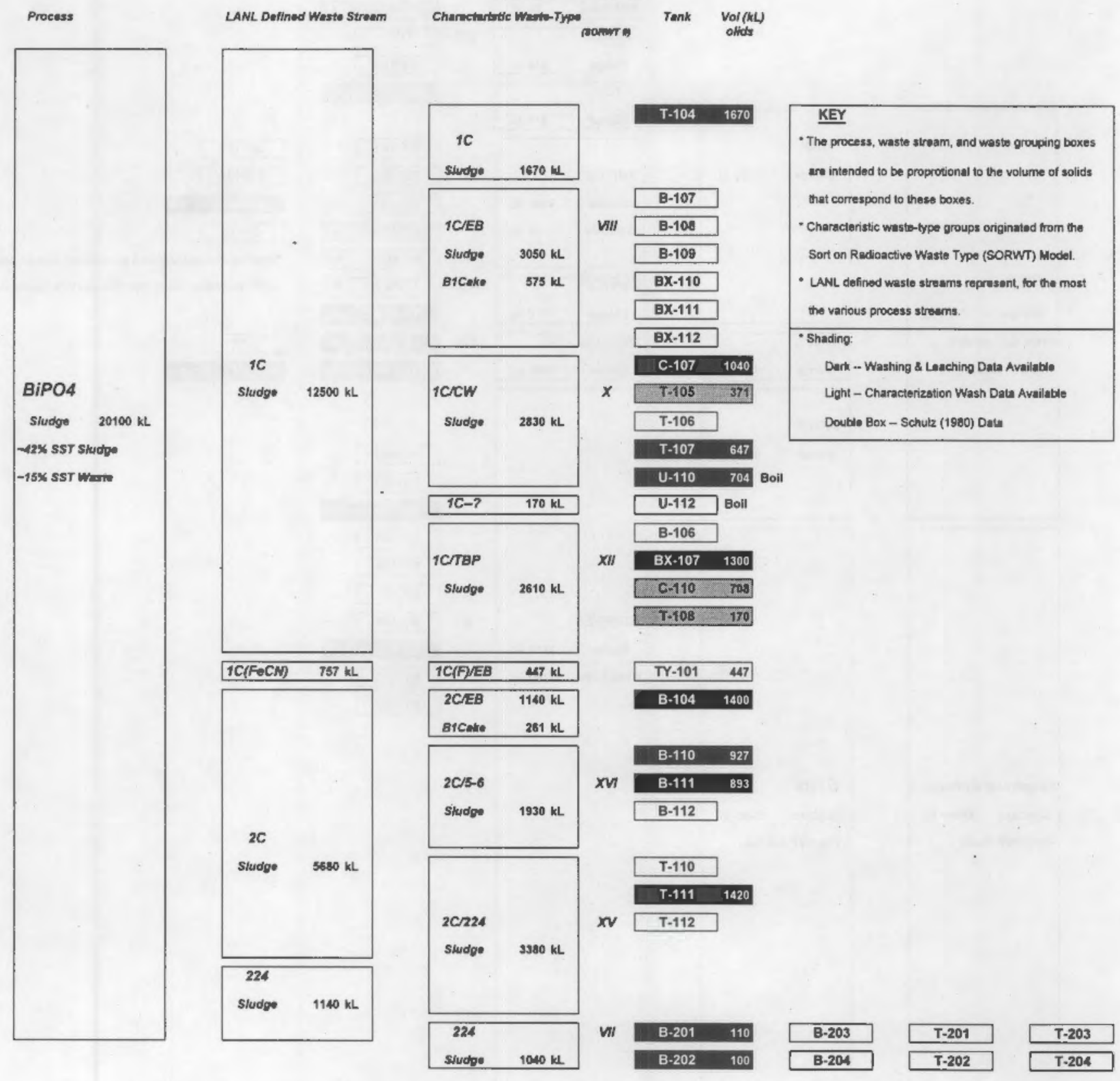

Figure 1. Pictorial Summary of Relationships Among Chemical Processes/Campaigns, Waste Streams, Characteristic Waste-type Groups, and Single-shell Tanks 
Process

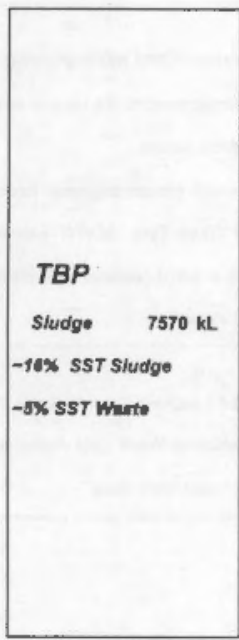

Evaporator Bottoms: Sercanke $87400 \mathrm{~kL}$ -65\% SST Wate
LANL Defined Waste Strenm
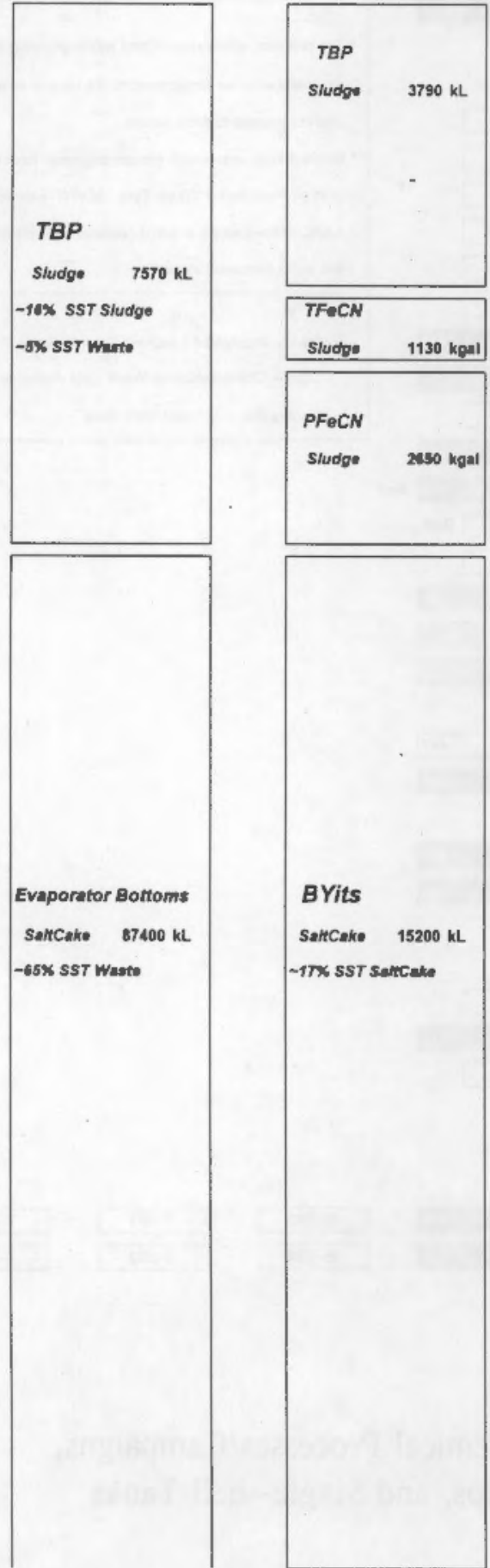

BYits

Senceke $15200 \mathrm{~kL}$

-Tr* SST Sencate

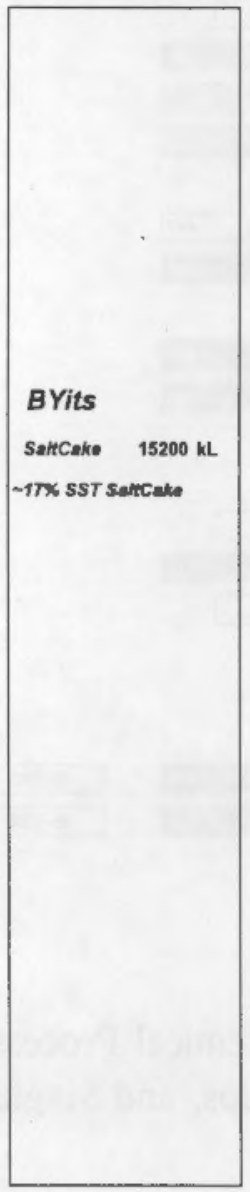

Chemeteriode Warto-Type

roment Tank Volf(ki)

\begin{tabular}{|c|c|}
\hline TEP/Sr & $570 \mathrm{~kL}$ \\
\hline TEPRDiaE & $H \mathrm{~kL}$ \\
\hline \multicolumn{2}{|l|}{ TAP/EB } \\
\hline Siudpe & $814 \mathrm{~kL}$ \\
\hline \multicolumn{2}{|l|}{$T E P$} \\
\hline Sludpe & $874 \mathrm{~kL}$ \\
\hline \multicolumn{2}{|l|}{$T B P / C W$} \\
\hline Studges & $2560 \mathrm{~kL}$ \\
\hline Sancake & $10 \mathrm{~kL}$ \\
\hline & \\
\hline
\end{tabular}

Siuctge $\quad 776 \mathrm{~kL}$

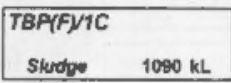

\begin{tabular}{|l|}
\hline C-105 34 \\
\hline
\end{tabular}

\begin{tabular}{|ll}
\hline$T r-100$ & 94
\end{tabular}

$x x$

\begin{tabular}{|c|}
\hline T-109 \\
\hline TX-103 \\
\hline
\end{tabular}

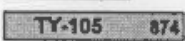

BX-101

v $\mathrm{BX}-102$

BX-103

BX-100 280

\begin{tabular}{|ll}
\hline $\mathrm{BX}-105^{*}$ & 160 \\
\hline
\end{tabular}

XXII $\quad \mathrm{TY}-103 \quad 613$

\begin{tabular}{|l|}
\hline TY.104 160 \\
\hline
\end{tabular}

XIII C-108

C-109 230

\begin{tabular}{l|}
\hline BX-106 \\
\hline BX-108 \\
\hline BX-109 \\
\hline C.101 \\
\hline
\end{tabular}

Washing and leaching lests pertormed; however, not included

in this evaluation due to discrepancies in projectied inventories.

C-111

C-112
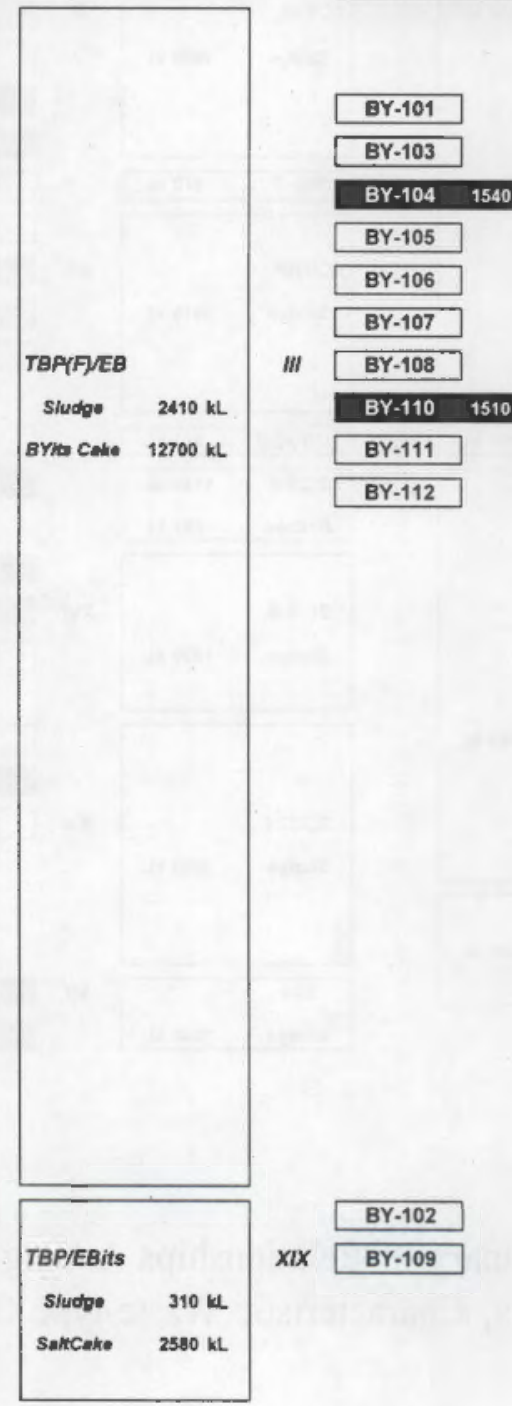

\begin{tabular}{ll} 
& BY-102 \\
\hline XIX & \\
\hline & BY-109 \\
\hline
\end{tabular} 


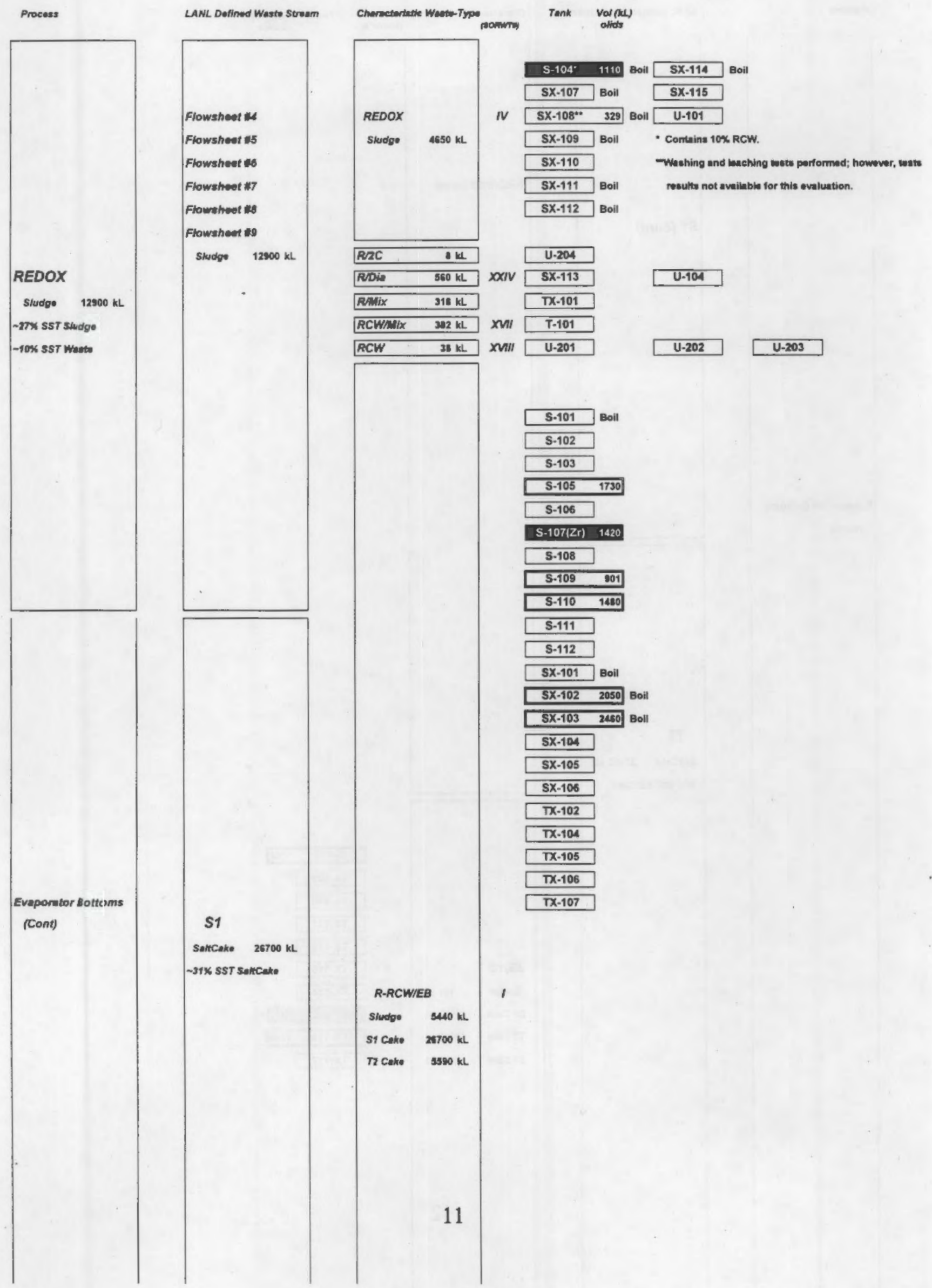


Process

LANL Defined Waste Stream

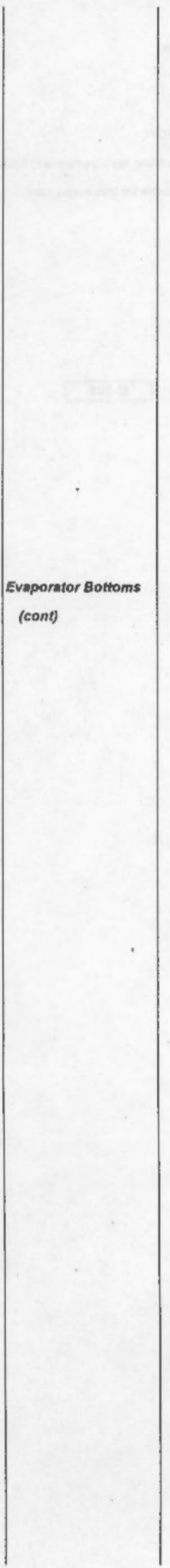

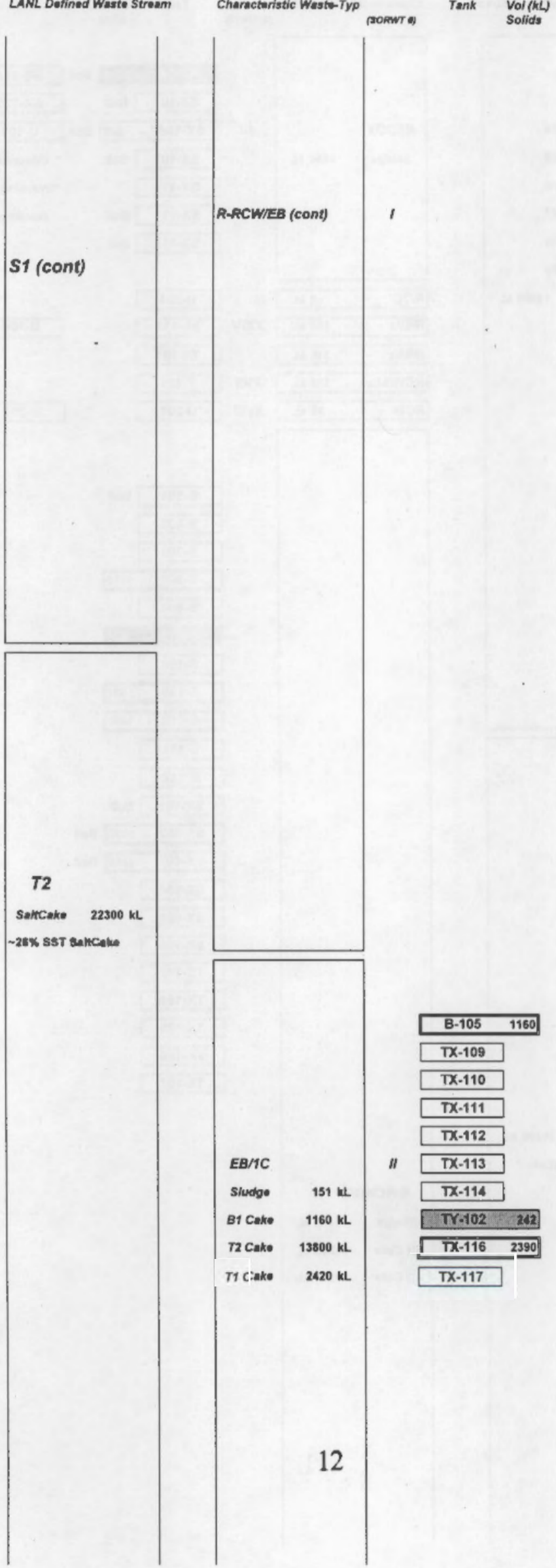




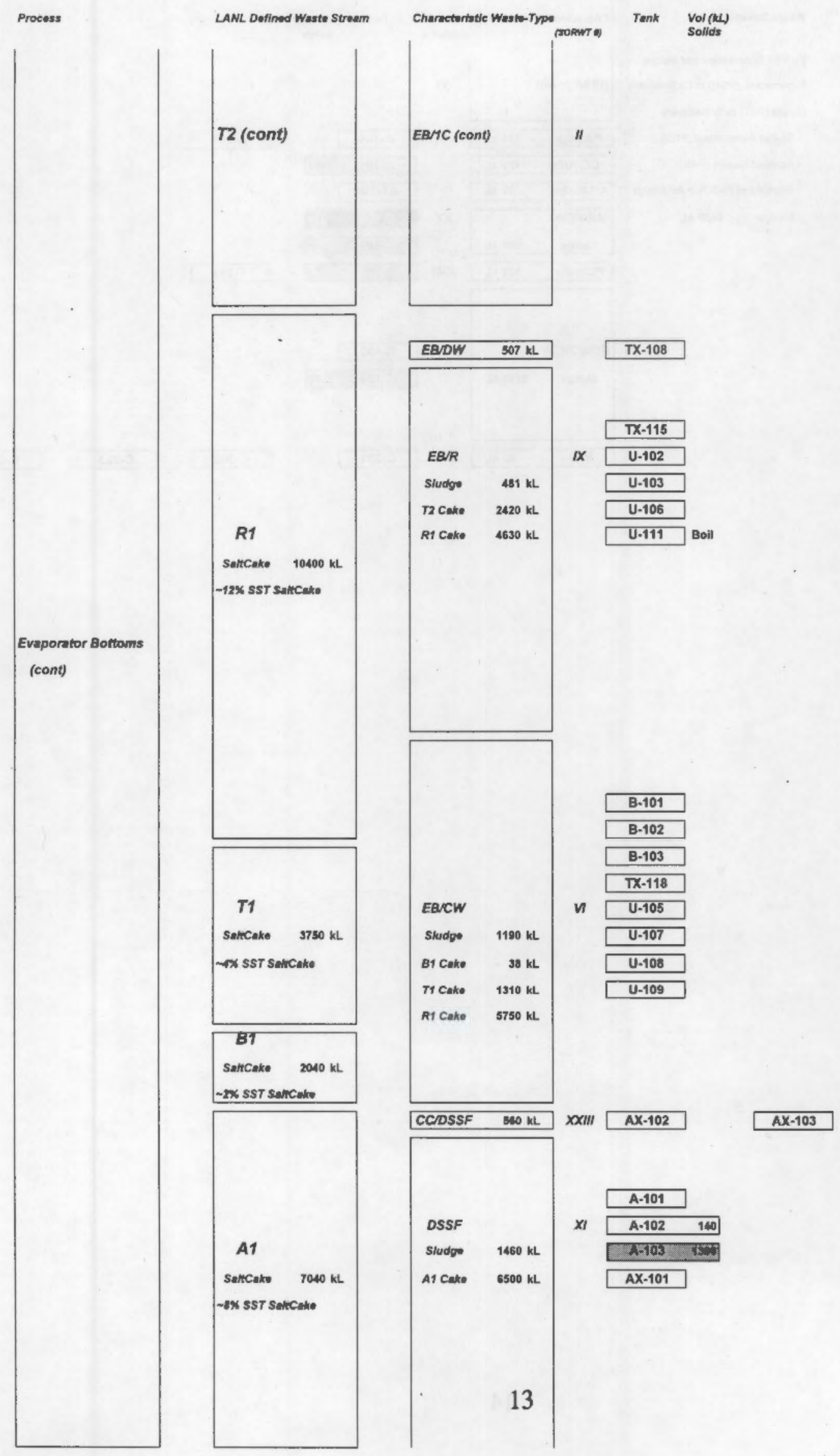



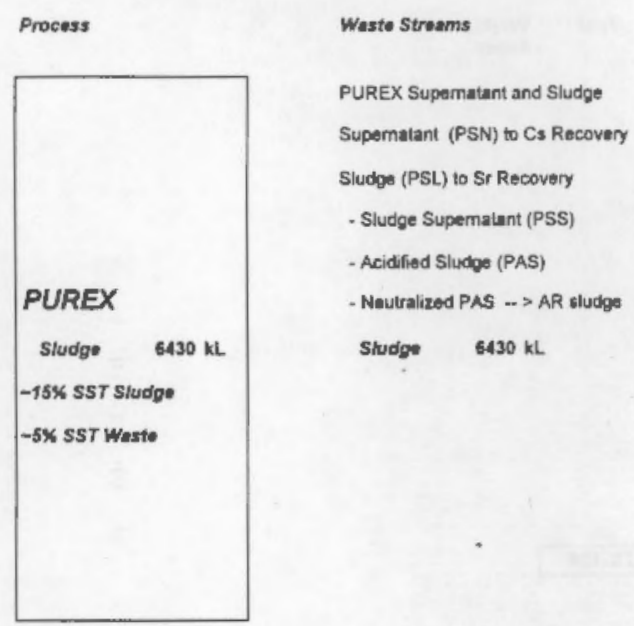

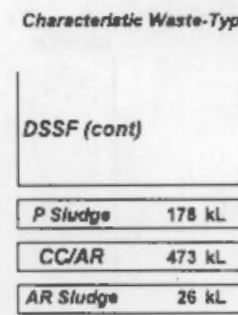

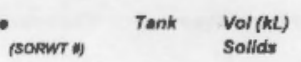

$x$

$x I$

\begin{tabular}{llllll}
$A R P C W$ & $X X \quad \mathrm{C}-103$ & 235 \\
\hline
\end{tabular}

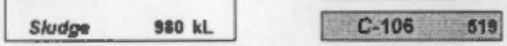

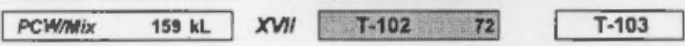

\begin{tabular}{|l|}
\hline PCW/ZrCW \\
Siudoe $2720 \mathrm{~kL}$ \\
\\
\end{tabular}

C.102

C-104 1120 


\subsection{Results}

Wash and leach factor evaluations have been completed for 16 nonradioactive analytes ( $\mathrm{Al}, \mathrm{Ba}, \mathrm{Bi}, \mathrm{Ca}, \mathrm{Cd}, \mathrm{Cr}, \mathrm{Fe}, \mathrm{Mg}, \mathrm{Mn}, \mathrm{Na}, \mathrm{Ni}, \mathrm{PO}_{4}, \mathrm{Si}, \mathrm{Sr}, \mathrm{U}$, and $\mathrm{Zr}$ ) and 2 radioactive analytes $\left({ }^{137} \mathrm{Cs}\right.$ and $\left.{ }^{90} \mathrm{Sr}\right)$. The results from these extensive evaluations are detailed in analyte worksheets at the end of this section. These worksheets show the calculated distribution of a particular analyte (in metric tons) among the wash and leach steps and in the residual solids. An analyte-specific wash factor (\% analyte removed from initial solids) and leach factor (\% analyte removed from washed solids) also have been calculated.

Examples of additional information that can be readily extracted from these analyte worksheets are listed below:

- tanks that have an unusually high inventory of a particular analyte; e.g., C-112 has a very high inventory of uranium.

- characteristic wastes groups that contain a large fraction of a particular analyte inventory; e.g., half the sodium inventory may be concentrated in 22 tanks that contain Redox/Evaporator Bottom wastes (SORWT Group I).

- characteristic waste groups that require more sampling/testing because of their significant bearing on TPA Milestone M50-03 (the 1998 decision regarding the level of pretreatment to be incorporated into the tank waste process flowsheets), e.g., Redox/Evaporator Bottom, and Double-shell Slurry Feed wastes that potentially have large concentrations of chromium remaining in the residual solids (after washing and leaching).

The wash and leach factors resulting from these evaluations are summarized in Table 3. These factors will be incorporated into the TWRS Process Technical Baseline, where the wash factors will be used to partition the SST inventory into soluble and insoluble portions, and the leach factors will be used to further remove analytes, such as sodium, aluminum, chromium, phosphate, and other minor components. 
Table 3. Summary of Wash and Leach Factors for a Composite

Single-shell Tank Waste Inventory

\begin{tabular}{|c|c|c|c|c|c|}
\hline Analyte & $\begin{array}{l}\text { Wash Factor } \\
\text { (\% analyte } \\
\text { removed from } \\
\text { initial solids) }\end{array}$ & $\begin{array}{l}\text { Leach Factor } \\
\text { ( } \% \text { analyte } \\
\text { removed from } \\
\text { washed solids) }\end{array}$ & Analyte & $\begin{array}{l}\text { Wash Factor } \\
\text { (\% analyte } \\
\text { removed from } \\
\text { initial solids) }\end{array}$ & $\begin{array}{l}\text { Leach Factor } \\
\text { (\% analyte } \\
\text { removed from } \\
\text { washed solids) }\end{array}$ \\
\hline $\mathrm{Al}$ & 31 & 60 & $\mathrm{Mn}$ & $<1$ & $\sim 0$ \\
\hline $\mathrm{Ba}$ & 1 & $\sim 0$ & $\mathrm{Na}$ & $\begin{array}{l}95^{(\boldsymbol{(})} \\
94^{(\mathrm{c})}\end{array}$ & $\begin{array}{l}45^{(a)} \\
46^{(c)}\end{array}$ \\
\hline $\mathrm{Bi}$ & 0.3 & 0.3 & $\mathrm{Ni}$ & $2-5$ & $\sim 0$ \\
\hline $\mathrm{Ca}$ & 4 & 2 & $\mathrm{PO}_{4}$ & $\begin{array}{l}76^{(())} \\
75^{(c)}\end{array}$ & $\begin{array}{l}70^{(a)} \\
70^{(c)}\end{array}$ \\
\hline Cd & 15 & $<1$ & $\mathrm{Si}$ & 12 & 13 \\
\hline $\mathrm{Cr}$ & $\begin{array}{l}61^{(\text {() }} \\
59^{(\mathrm{b})}\end{array}$ & $\begin{array}{l}10^{(a)} \\
14^{(b)}\end{array}$ & $\mathrm{Sr}$ & $<1$ & $\sim 0$ \\
\hline${ }^{137} \mathrm{Cs}$ & $\begin{array}{l}60^{(\mathrm{a})} \\
59^{(\mathrm{c})}\end{array}$ & $\begin{array}{l}60^{(a)} \\
61^{(c)}\end{array}$ & ${ }^{90} \mathrm{Sr}$ & $<1$ & 0.1 \\
\hline $\mathrm{Fe}$ & 0.4 & 0.2 & $\mathrm{U}$ & 2 & 2 \\
\hline $\mathrm{Mg}$ & 1 & 0.6 & $\mathrm{Zr}$ & 0.3 & $\sim 0$ \\
\hline
\end{tabular}

(a) Factors presented in June 30, 1996 draft report.

(b) As a result of technical review comments, chromium was reevaluated based on partitioning the Uranium Recovery/Evaporator Bottom waste group into a high chromium concentration waste group, i.e., BY-104 and BY-110 tank wastes, and a low chromium concentration waste group. Also, the density of salt cake was assumed to be $1.7 \mathrm{~g} / \mathrm{mL}$ rather than $1.9 \mathrm{~g} / \mathrm{mL}$.

(c) As a result of technical review comments, the density of salt cake was assumed to be 1.7 $\mathrm{g} / \mathrm{mL}$ rather than $1.9 \mathrm{~g} / \mathrm{mL}$. This change in density affected some partitioning factors. 


\section{Analyte Wash and Leach Factor Evaluations}


Analyte Wash and Leach Factor Evaluations 
Element: $A I$

BiPO4

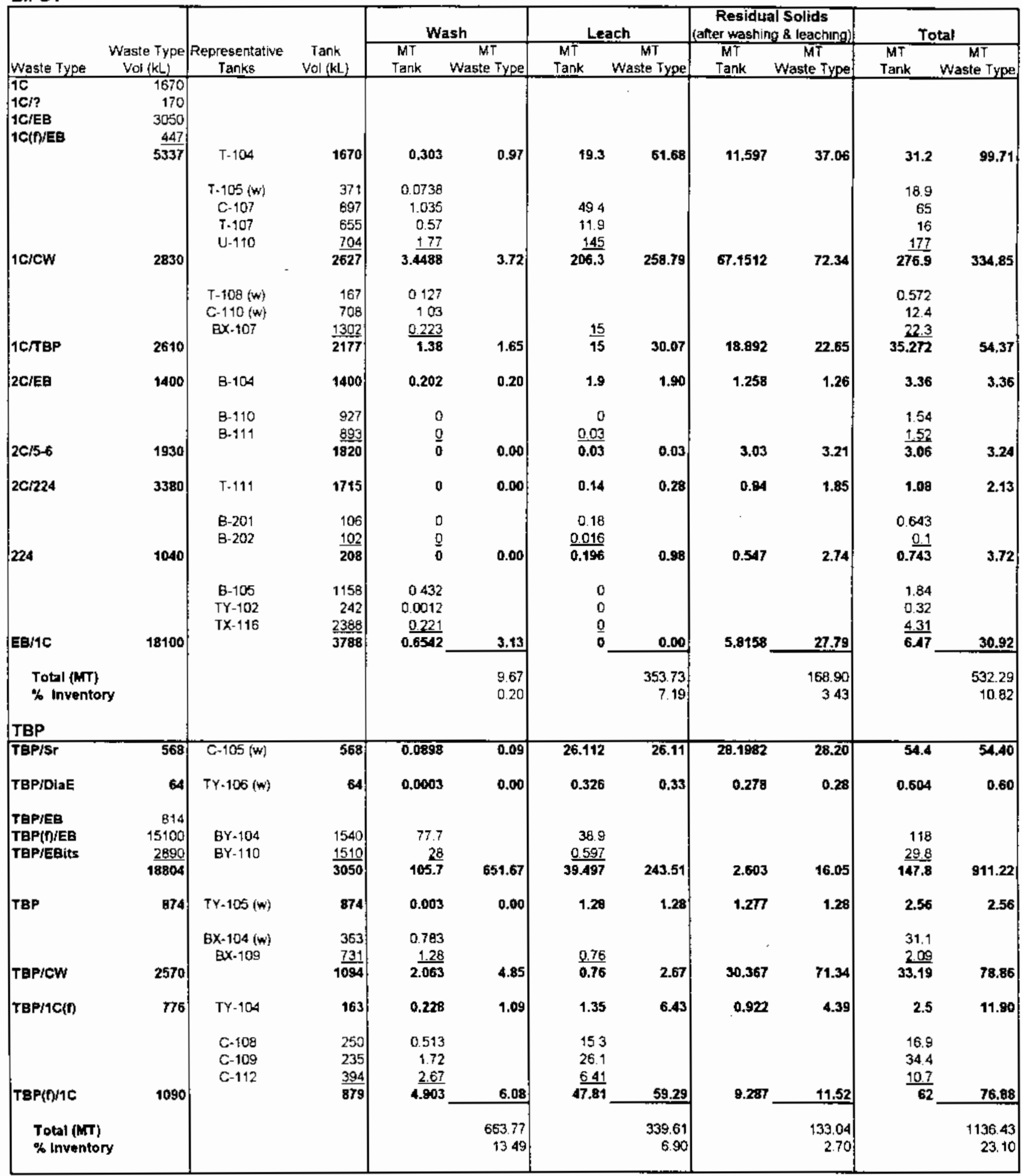


Element: Al (Continued)

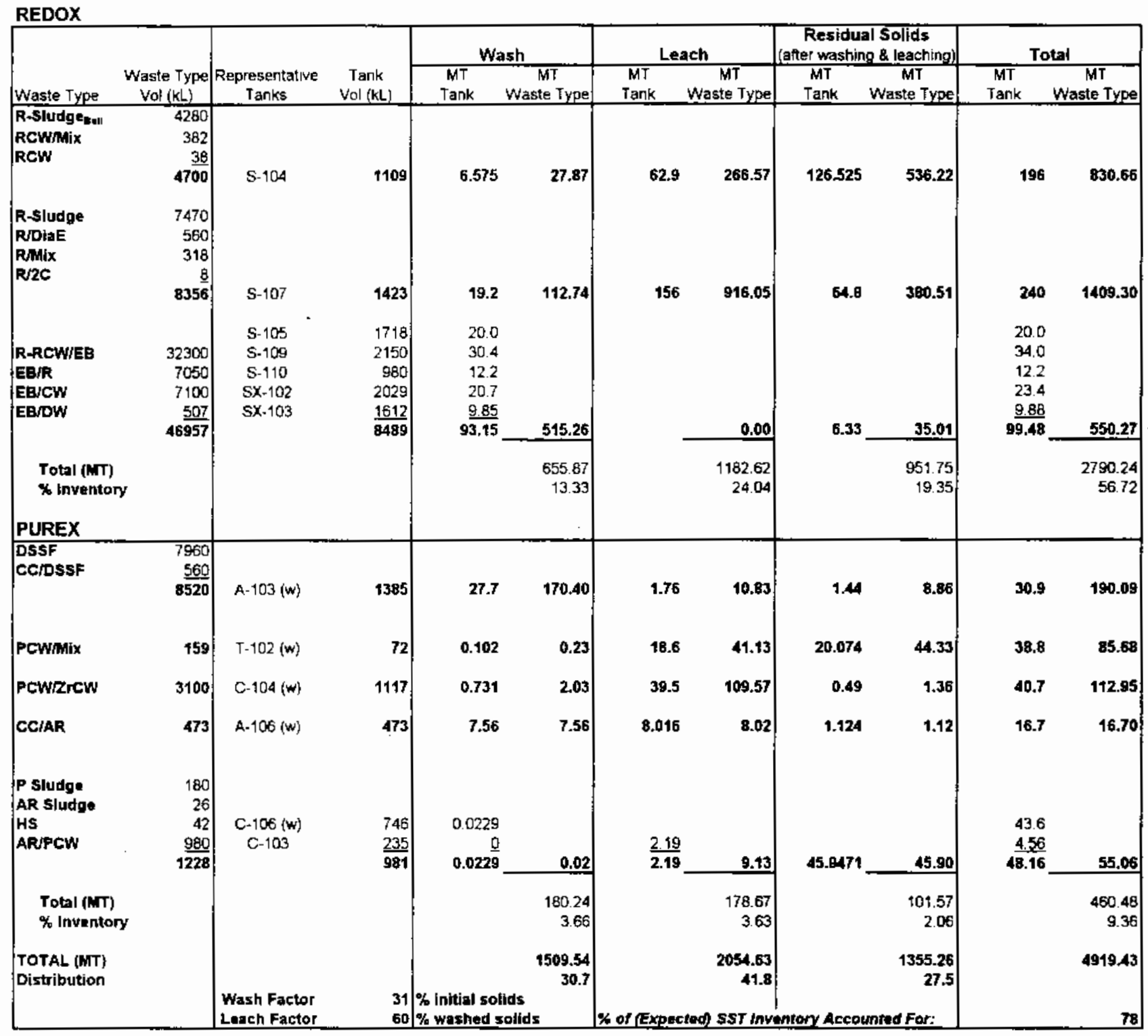

Leach factor from tests performed wth ARJPCW (Purnx) waste (C-103) used for DSSF, CCJDSSF, PCWMAlx, PCWR CW, and CCIAR (Purex) wartes.

\begin{tabular}{|c|c|}
\hline $\begin{array}{l}\text { "Provisional" Best-basis Tatal } \\
\text { Invertory (on-going work at WHC) } \\
\text { less TWRS QST inventory }\end{array}$ & $\begin{array}{c}\text { (MT) } \\
7850 \\
\text {-1570 }^{*}\end{array}$ \\
\hline $\begin{array}{l}\text { Expected } \$ S T \text { inventory } \\
\text { This Evaluation }\end{array}$ & $\begin{array}{l}6280 \\
4920\end{array}$ \\
\hline Expected Inventory Accounted for & $78 \%$ \\
\hline \multicolumn{2}{|c|}{$\begin{array}{l}\text { "LANL estimates } 2190 \text { MT In DSTS. Polsntially more than expacted } \\
\text { in DSTs or m/ssing representatlye tanks for AI in thils analysis. }\end{array}$} \\
\hline
\end{tabular}


Element: Ba

BiPO4

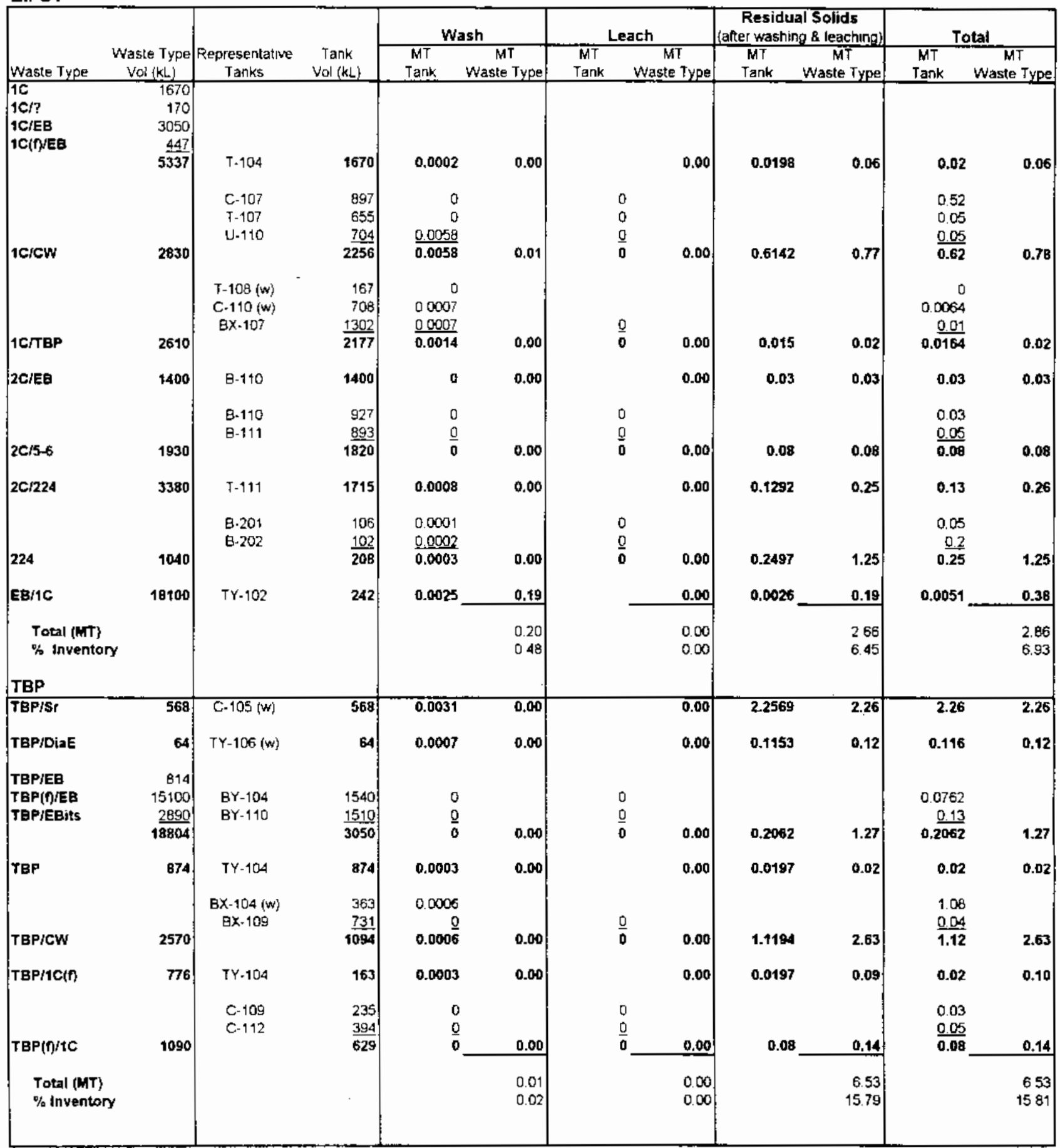


Element: Ba (Continued)

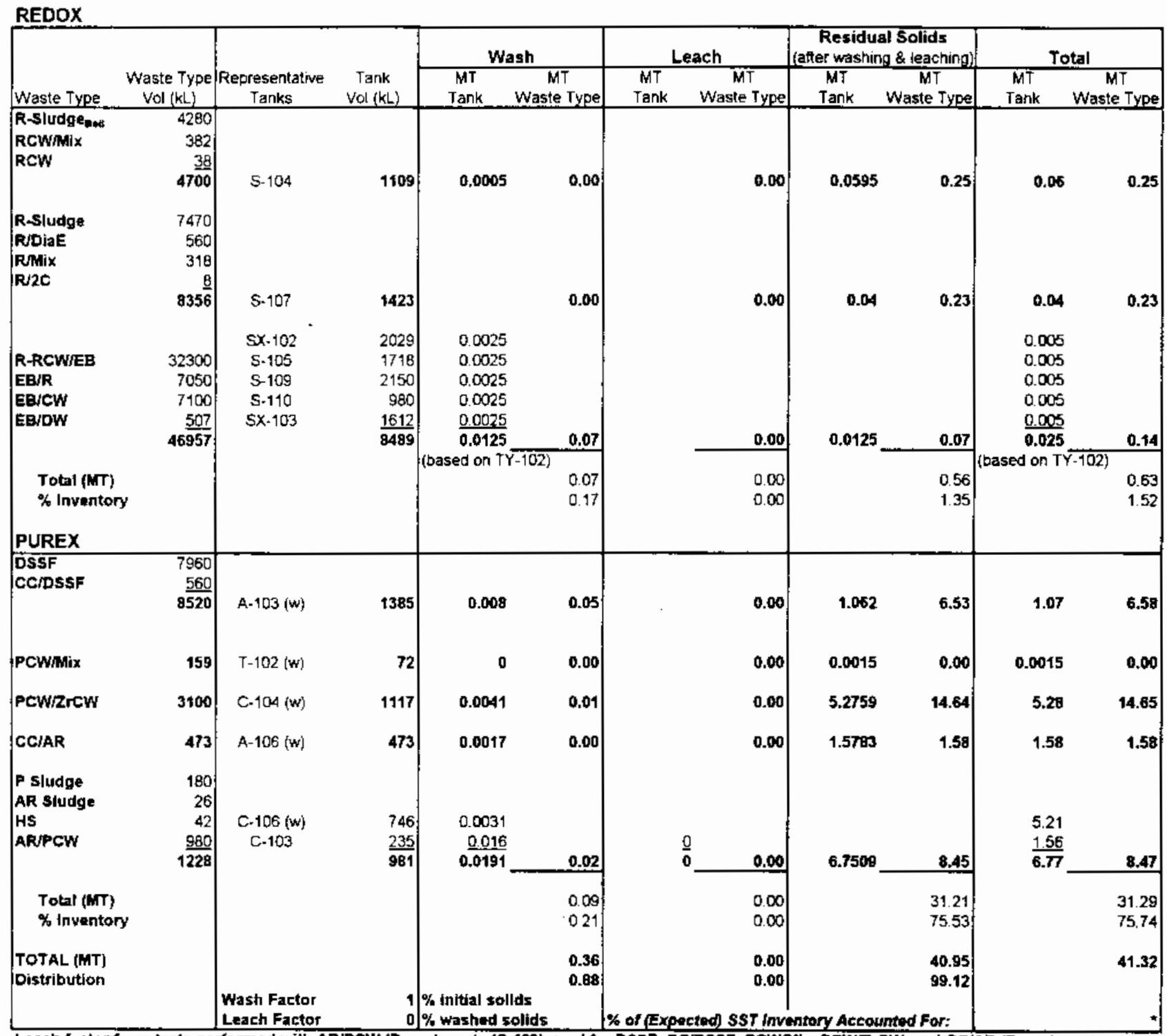

Leach factor from tests performed with AR/PCW (Purex) waste (C-103) used for DSSF, CCJDSSF, PCWMix, PCW/zrCW, and CCIAR (Purex) wastes.

*Ba inventory value not available. 
Element: $B i$

BiPO4

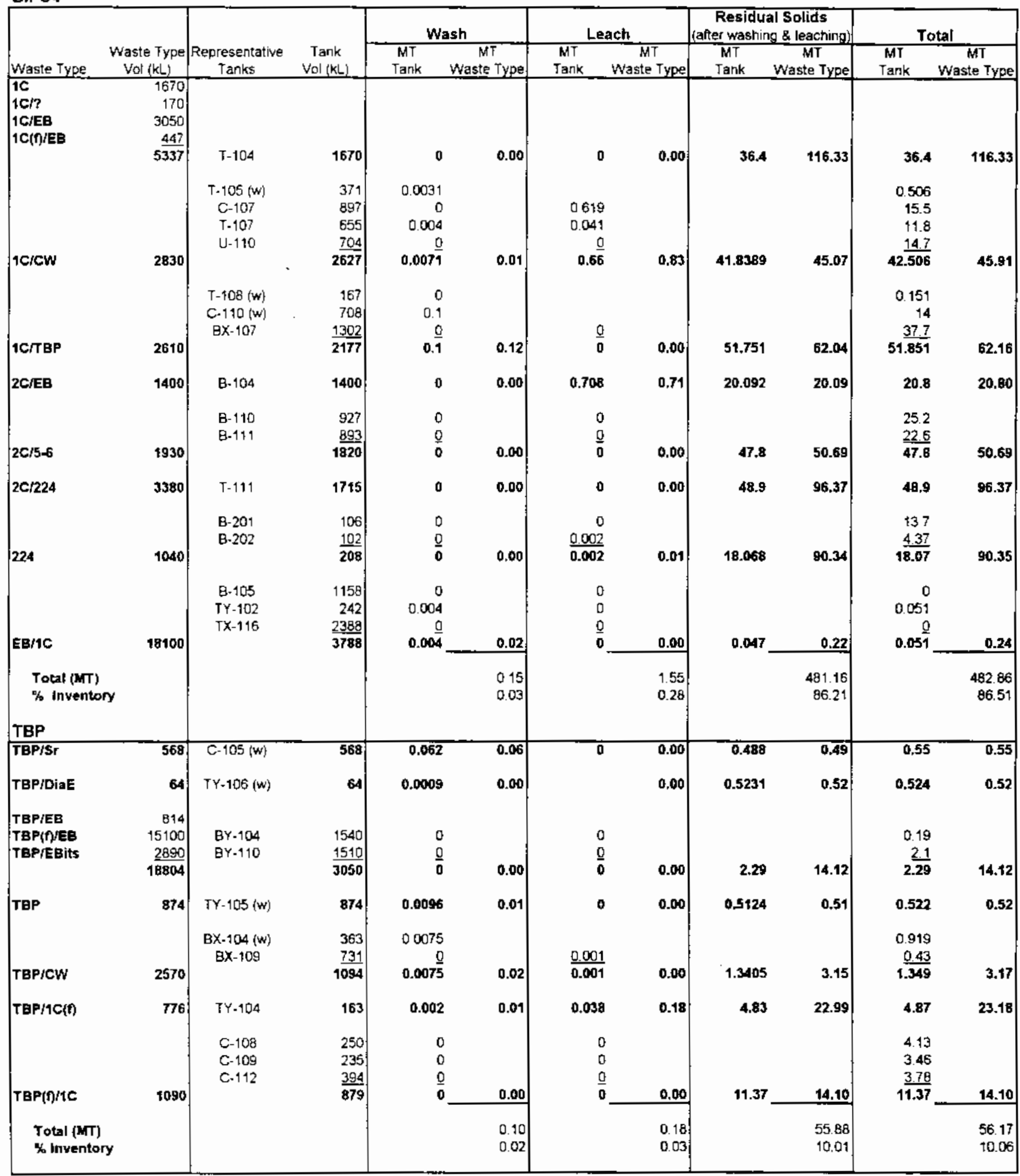


Element: Bi (Continued)

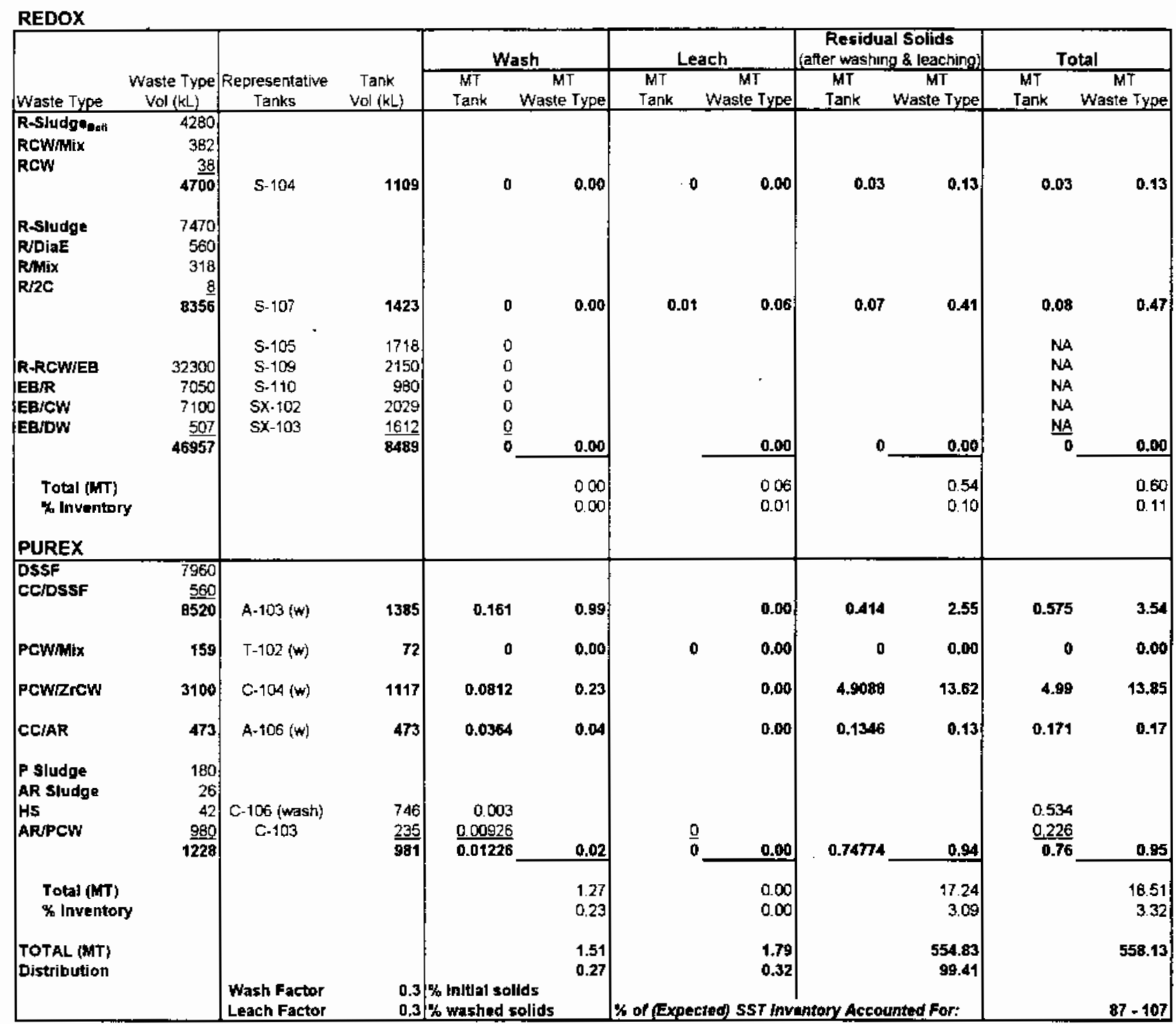

\begin{tabular}{|ll|}
\hline -Provisional" Best-basis Total & (MT) \\
Inventory (on-going work at WHC) & $580+1-60$ \\
less TWRS DST Inventory & $\underline{-2.2}$ \\
Expected SST Inventory & $578+1-60$ \\
This Eyaluation & 558 \\
Expected Inventory Accounted for & $97 \%$ \\
\hline
\end{tabular}


Eloment: $\mathrm{Ca}$

BiPO4

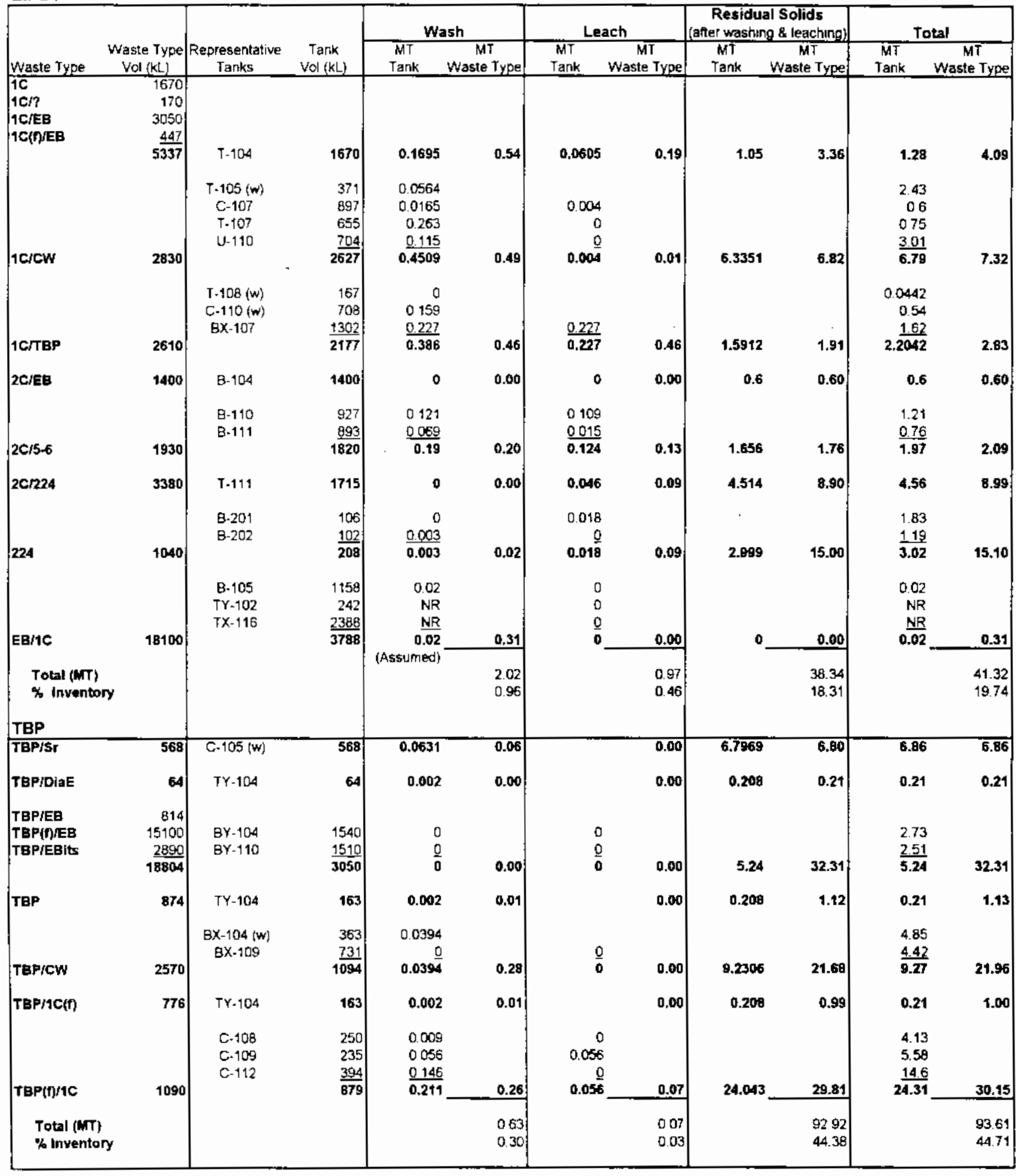




\section{Element: $\mathrm{Ca}$ (Continued)}

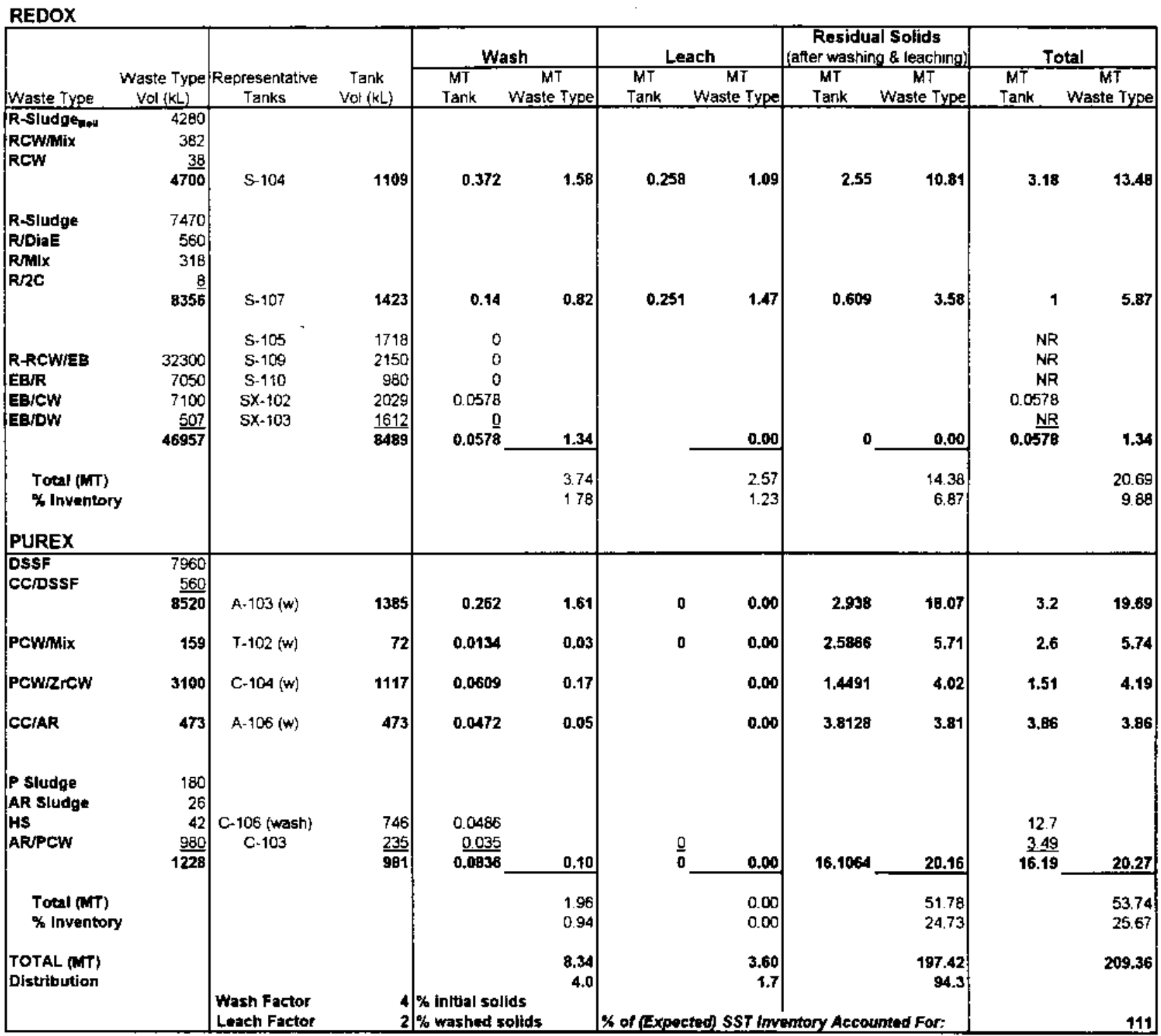

\begin{tabular}{|lc|}
\hline "Provisional" Eert-basis Total & (MT) \\
Inventory (on-going wort at WHC) & 214 \\
less TWRS DST Inventory & $-25,7$ \\
& 189 \\
Expected SST Inventory & 209 \\
Thls Evaluation & $111 \%$ \\
\hline
\end{tabular}


Element: Co

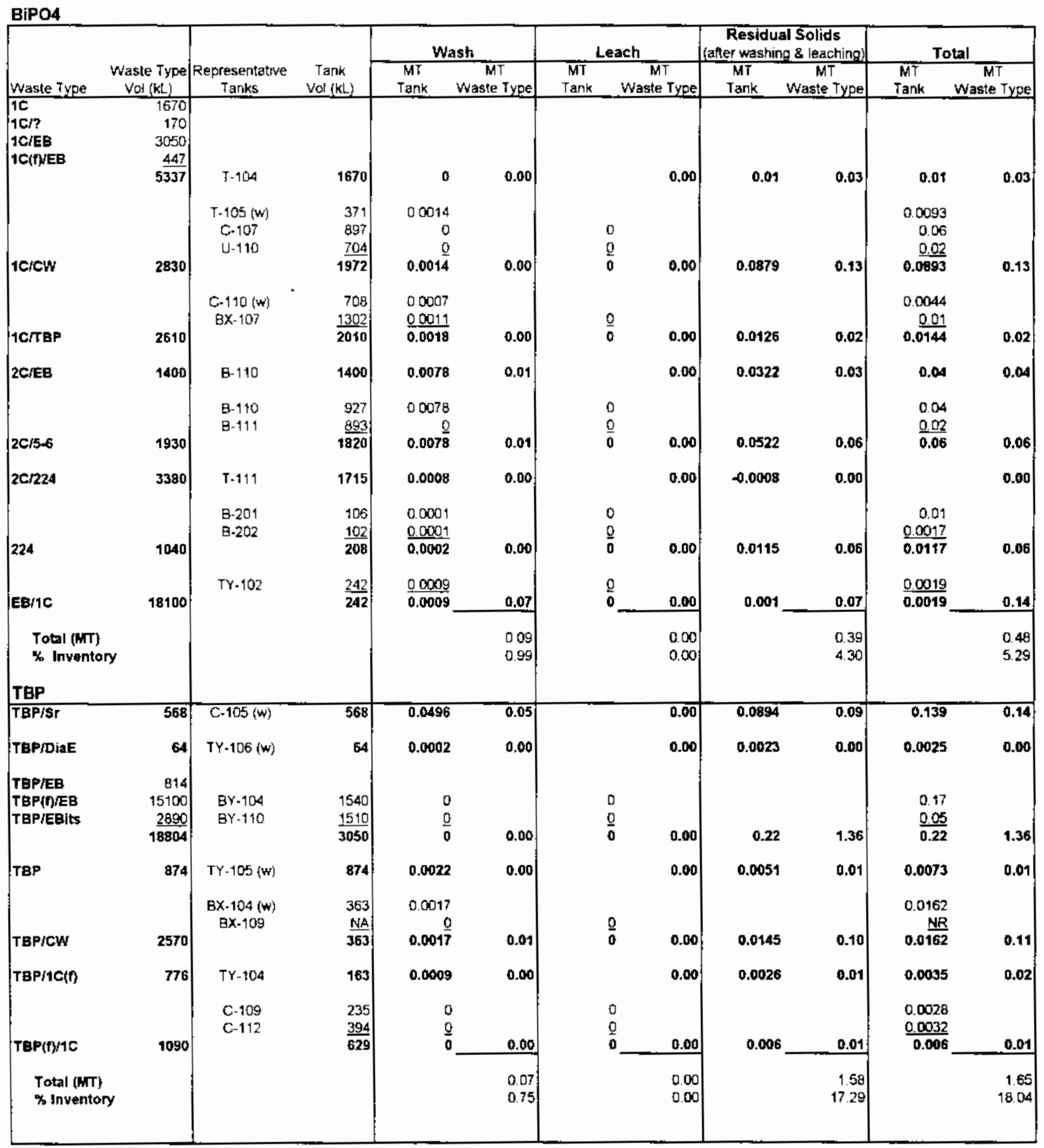


Element: Cd (Continued)

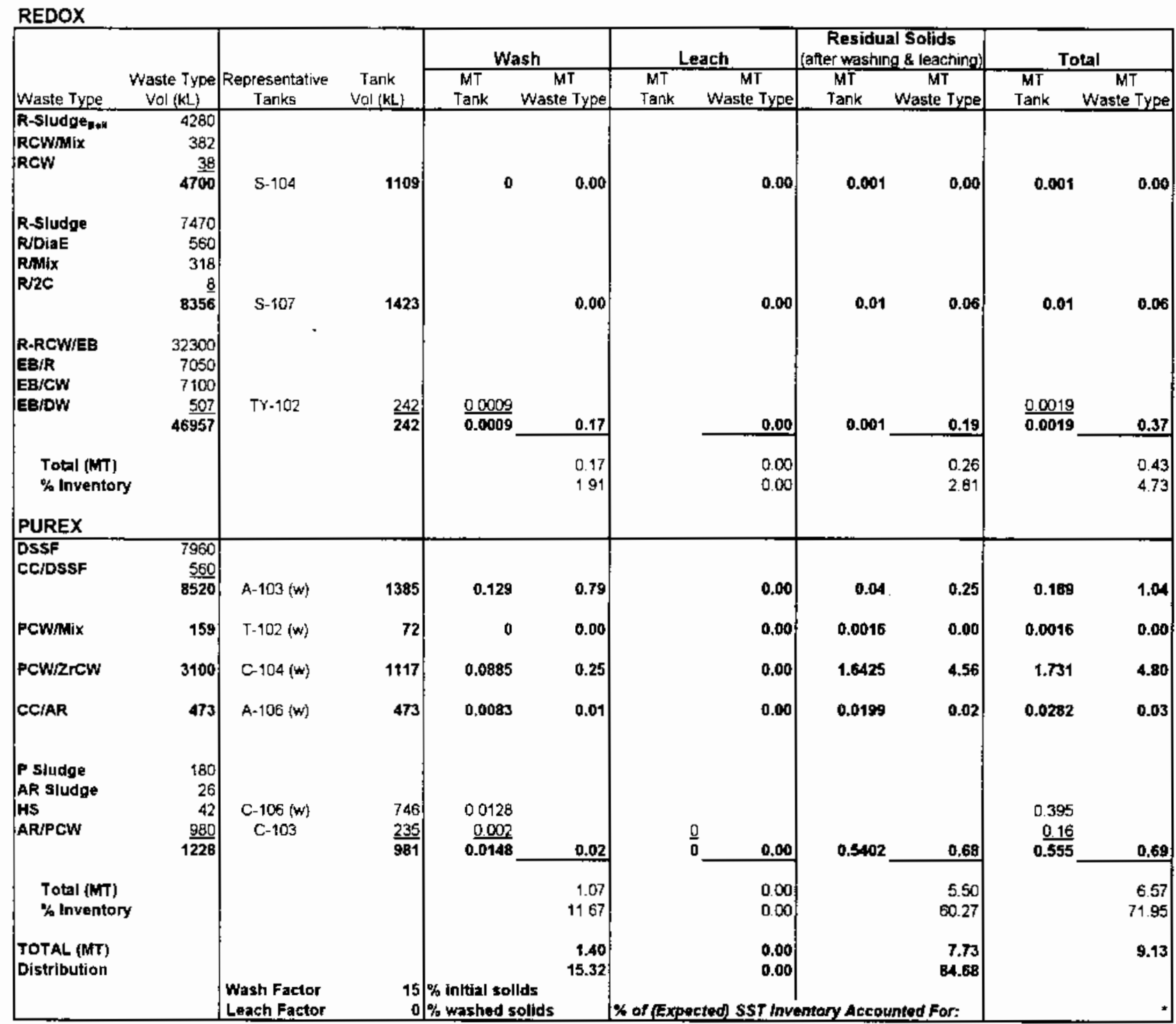


Element: $\mathrm{Cr}$

BiPO4

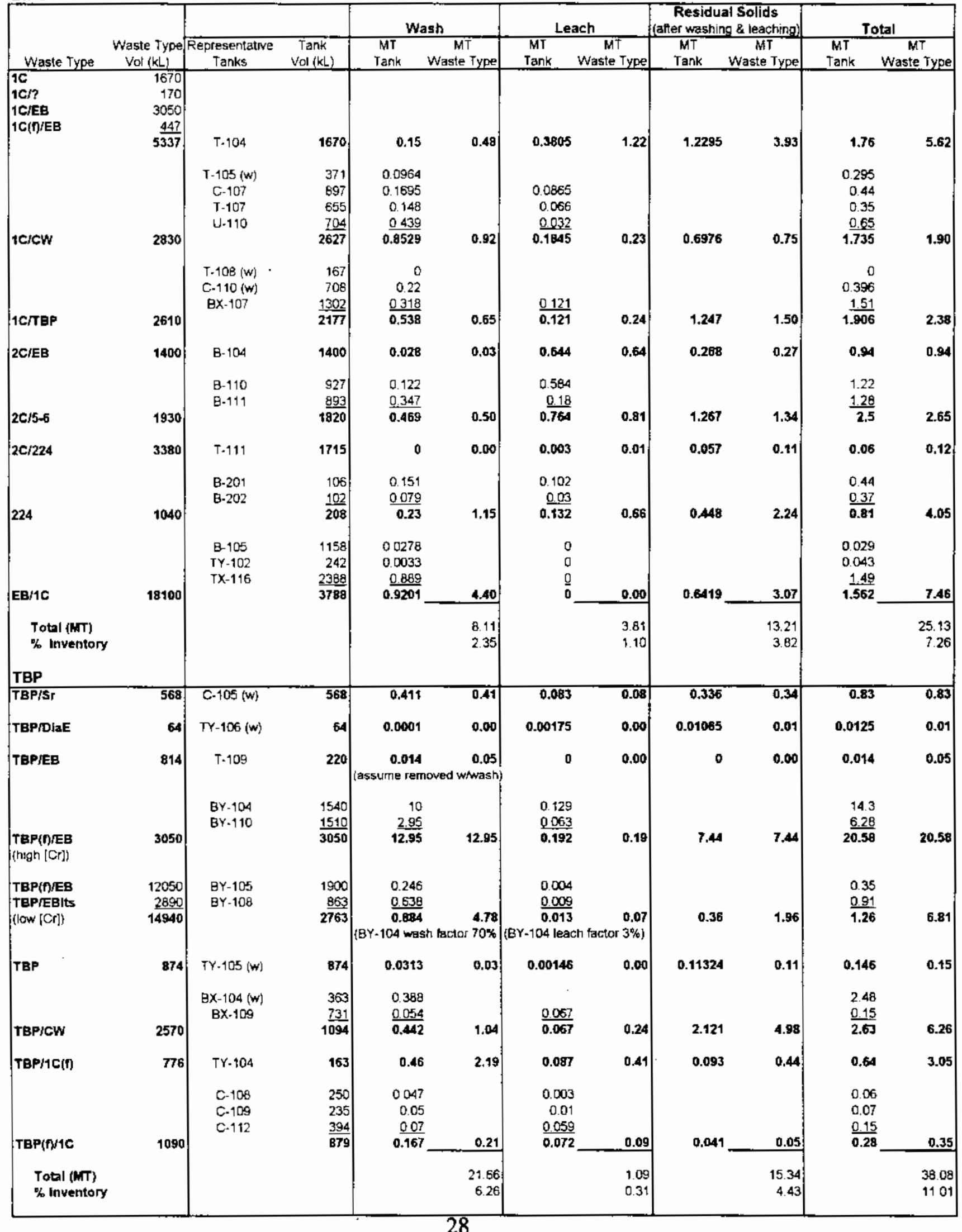


Element: $\operatorname{Cr}$ (Continued)

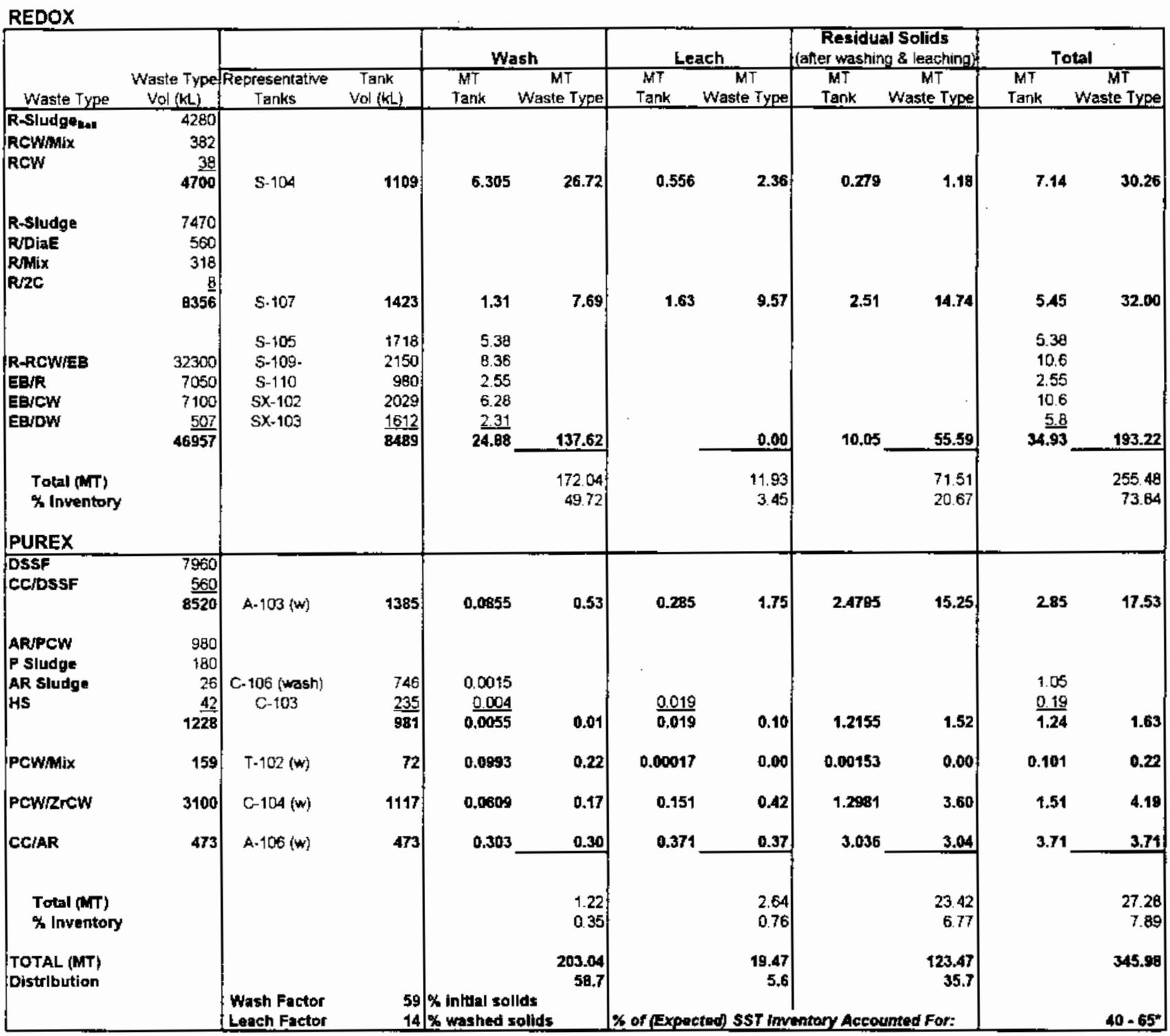

Leach factor hom torts performed wth ARPCW (Purux) wasto (C-103) used for DSSF, CCDSSF, PCWhaix, PCW/ZrCW, and CCIAR (PurnX) waster.

\begin{tabular}{|c|c|}
\hline \multirow[b]{2}{*}{$\begin{array}{l}\text { "Provisional" Best-basis Total } \\
\text { Inventory (on-going work at WHC) } \\
\text { less TWRS DST Inventory }\end{array}$} & (MT) \\
\hline & $\begin{array}{l}785+1-170^{\circ} \\
\text {-85 }\end{array}$ \\
\hline $\begin{array}{l}\text { Expected SST inventory } \\
\text { This Evaluation }\end{array}$ & $\begin{array}{l}700+1-170 \% \\
346\end{array}$ \\
\hline Expected Inventory Accoumlad for & $49 \%$ \\
\hline $\begin{array}{l}\text { " Uncerbinty In timing of changes to } \\
170 \text { MT uncertainty in the calculated }\end{array}$ & ults $\ln a$ \\
\hline
\end{tabular}


Element: ${ }^{137} \mathrm{Cs}$

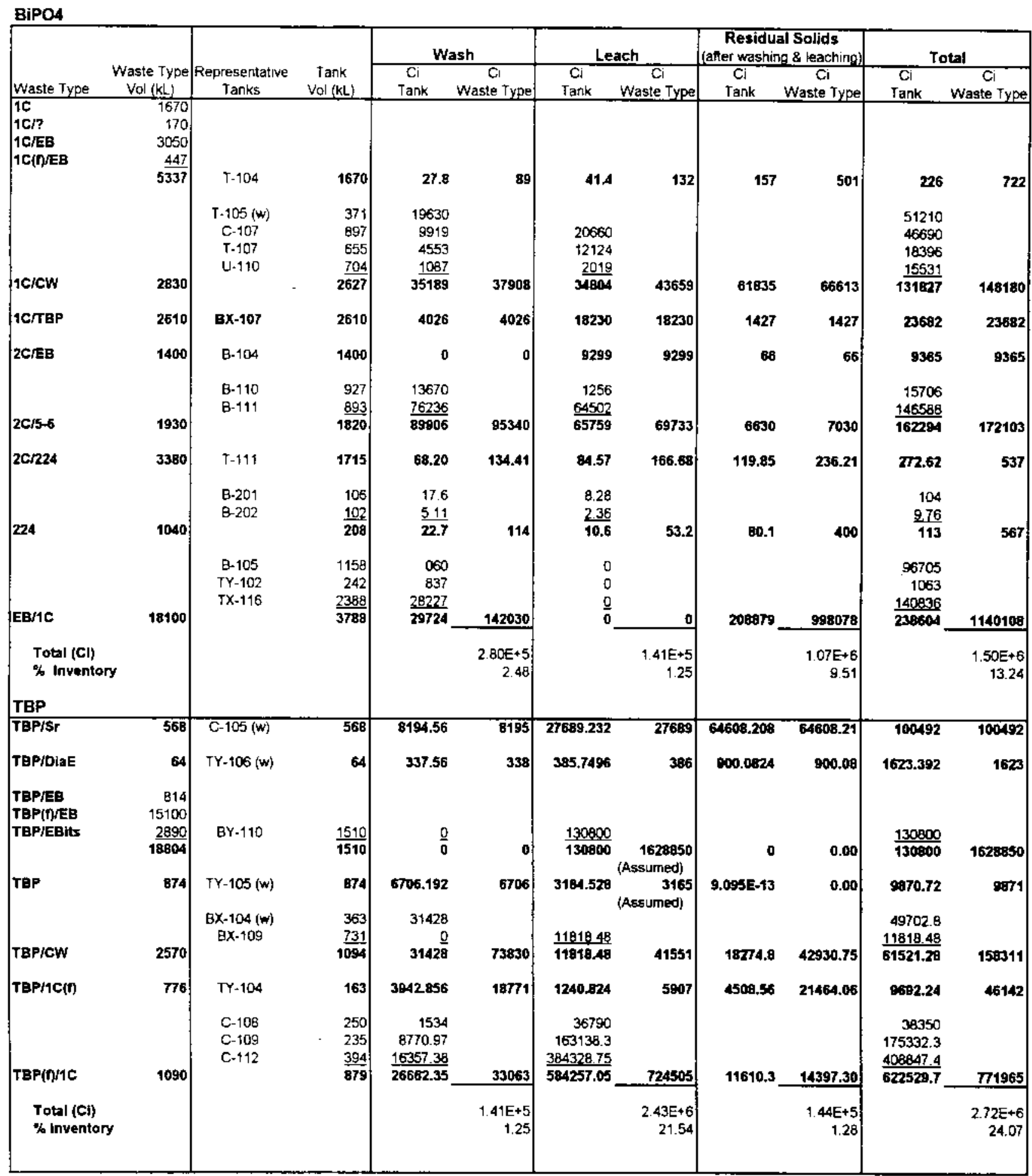


Element: ${ }^{47 /} \mathrm{Cs}$

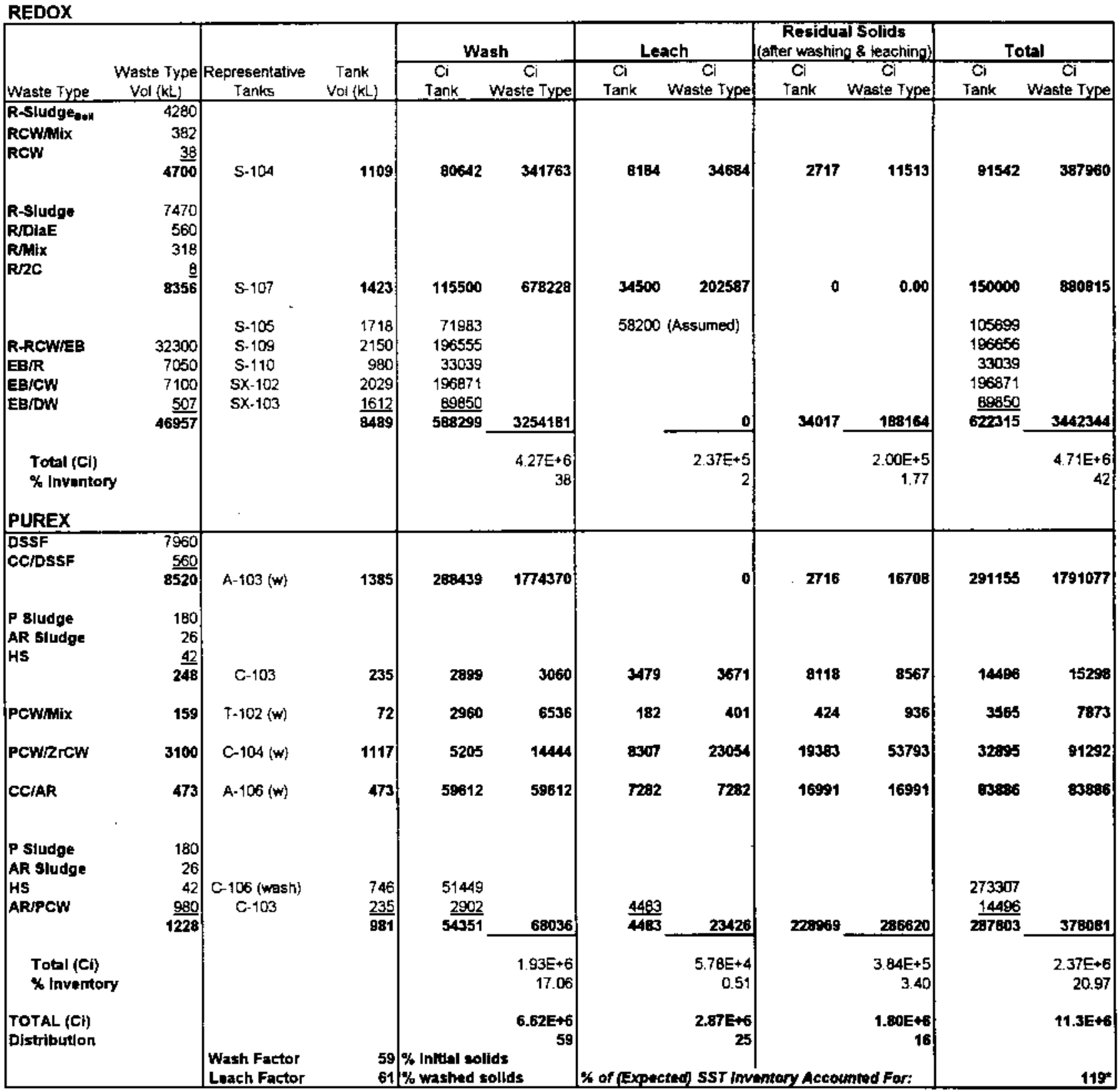

Leach factor trom tests pertormed wth ARPCW (Purnx) warte (C-103) used for DSSF, CCDSSF, PCWMIx, PCWRICW, and CCIAR (Purex) wastes.

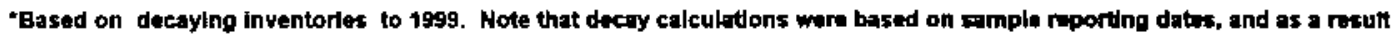
Inventorles are approximations.

\begin{tabular}{|lc|}
\hline "Provisional" Best-basis Total & (Ci) \\
Inventory (on-golng work at WHC) & $34.9 E+6$ \\
less TWRS DST Inventory & $\underline{-25.4 E+6}$ \\
& $09.5 \mathrm{E}+6$ \\
Expected \$ST Inventory & $11.3 \mathrm{E}+6$ \\
This Evaluation & $119 \%$ \\
\hline
\end{tabular}


Element: Fe

BiPO4

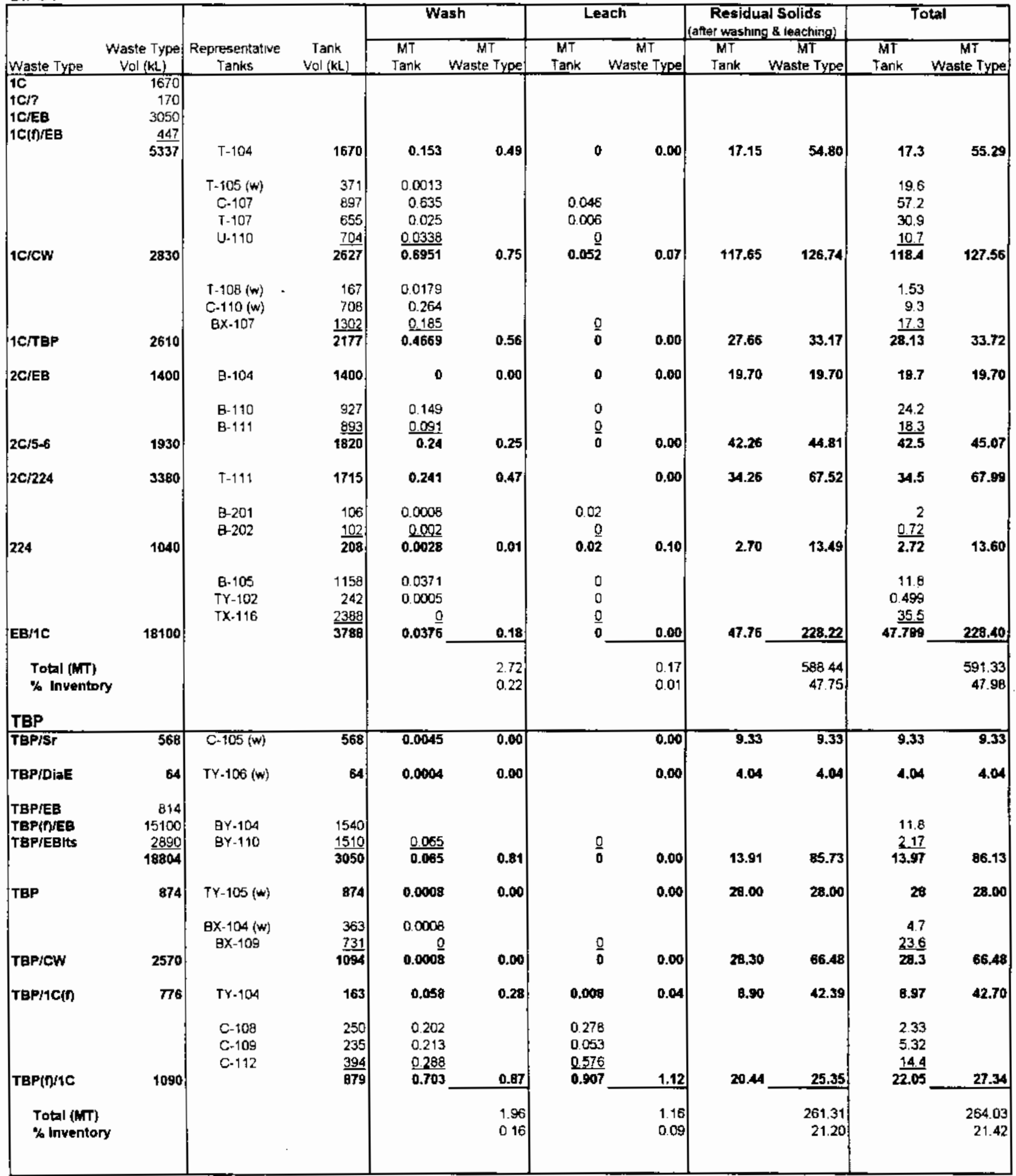


Element: Fe (Continued)

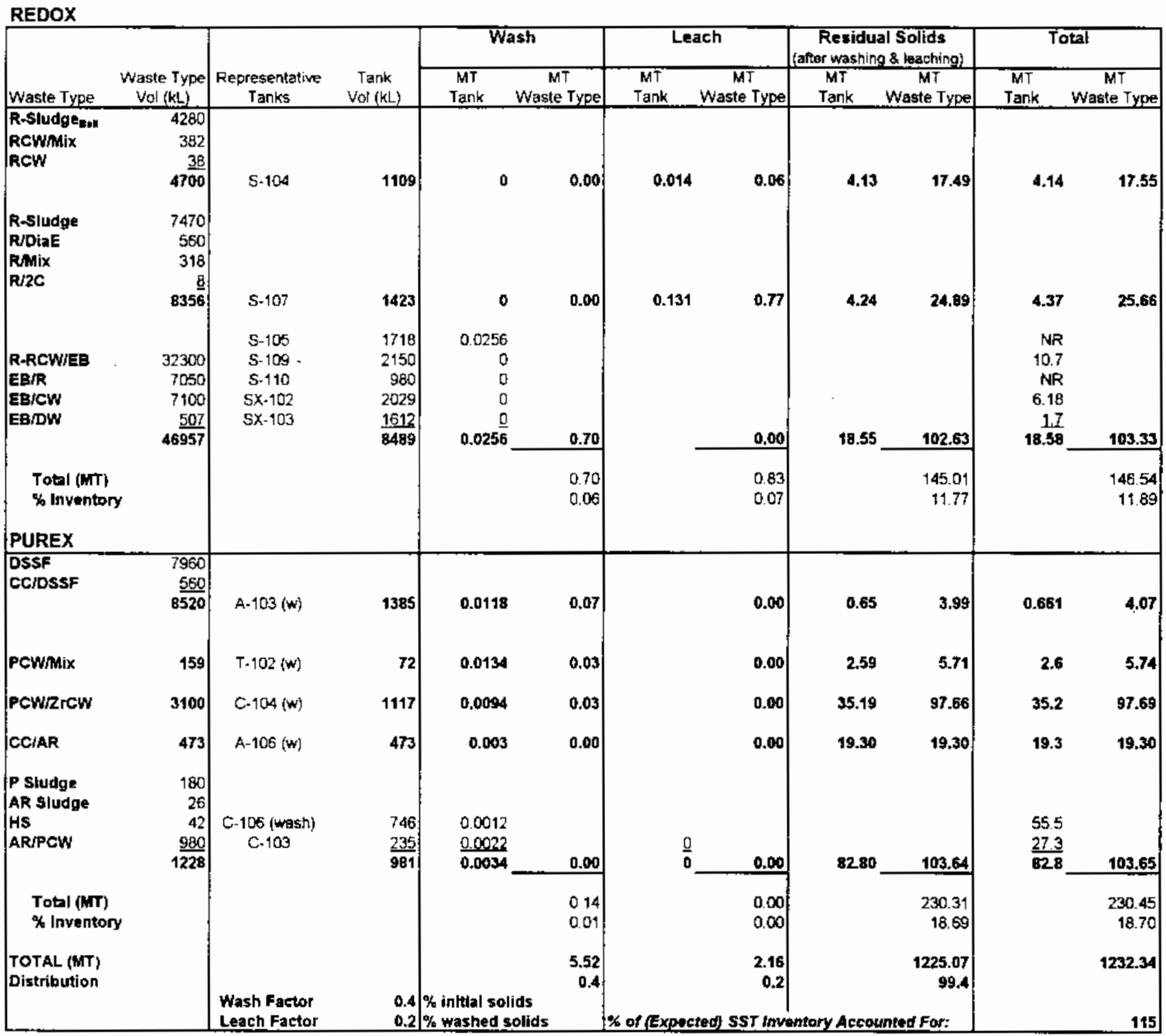

Leach factor from tests performed wh ARTPW (Purex) waste (C-103) used for DSSF, CCDSSF, PCWhilx, PCWZrCW, and CC/AR (Purex) wastes.

\begin{tabular}{|ll|}
\hline Provisional" Best-basis Total & (MT) \\
Invemory (on-going work at WHC) & 1230 \\
less TWRS DST Inventory & -162 \\
& 1068 \\
Expected SST Inventory & 1230 \\
This Evaluation & $115 \%$ \\
Expected Inventory Accountod for & \\
\hline
\end{tabular}


Element: $M g$

BiPO4

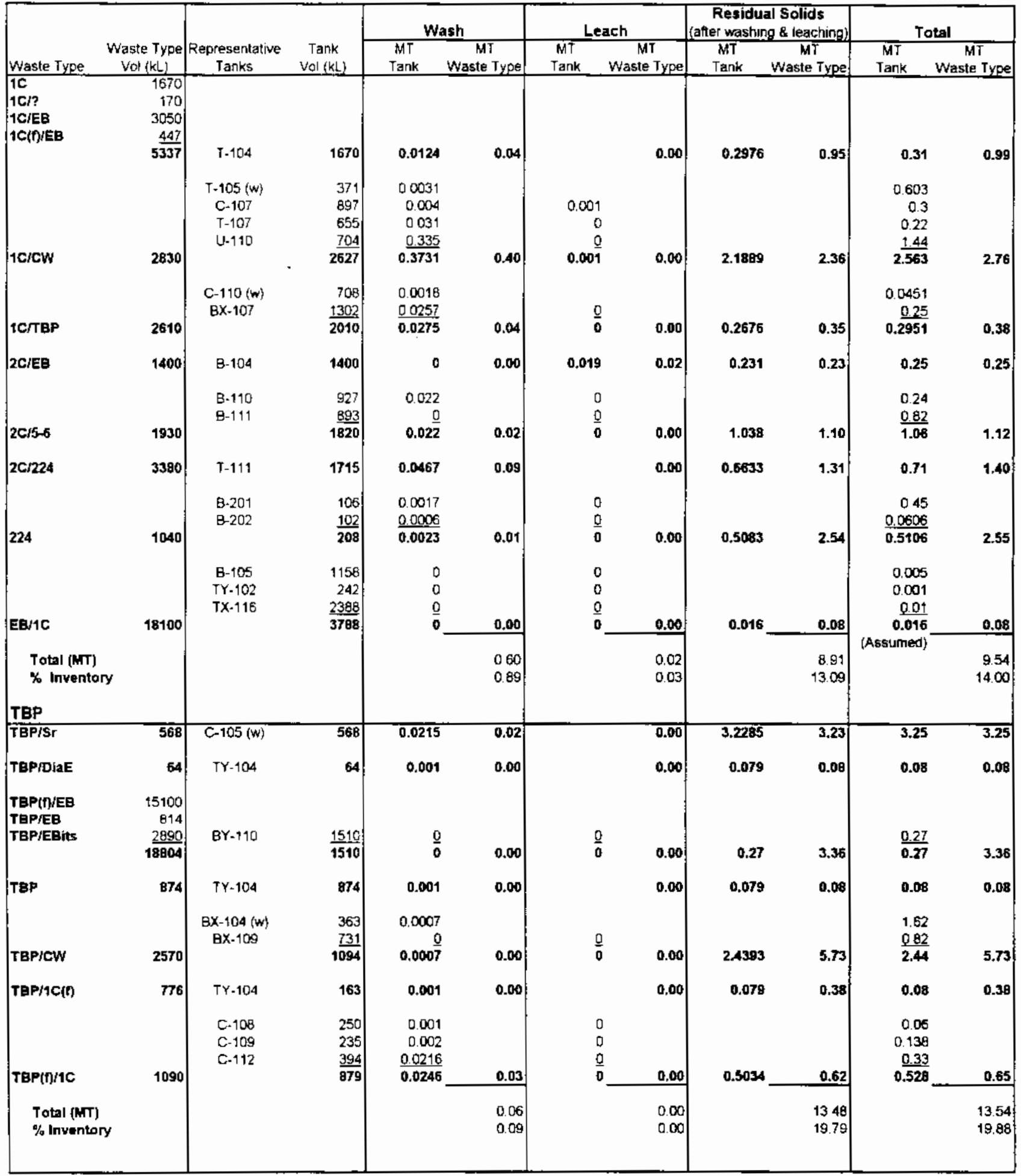


Element: $M g$ (Continued)

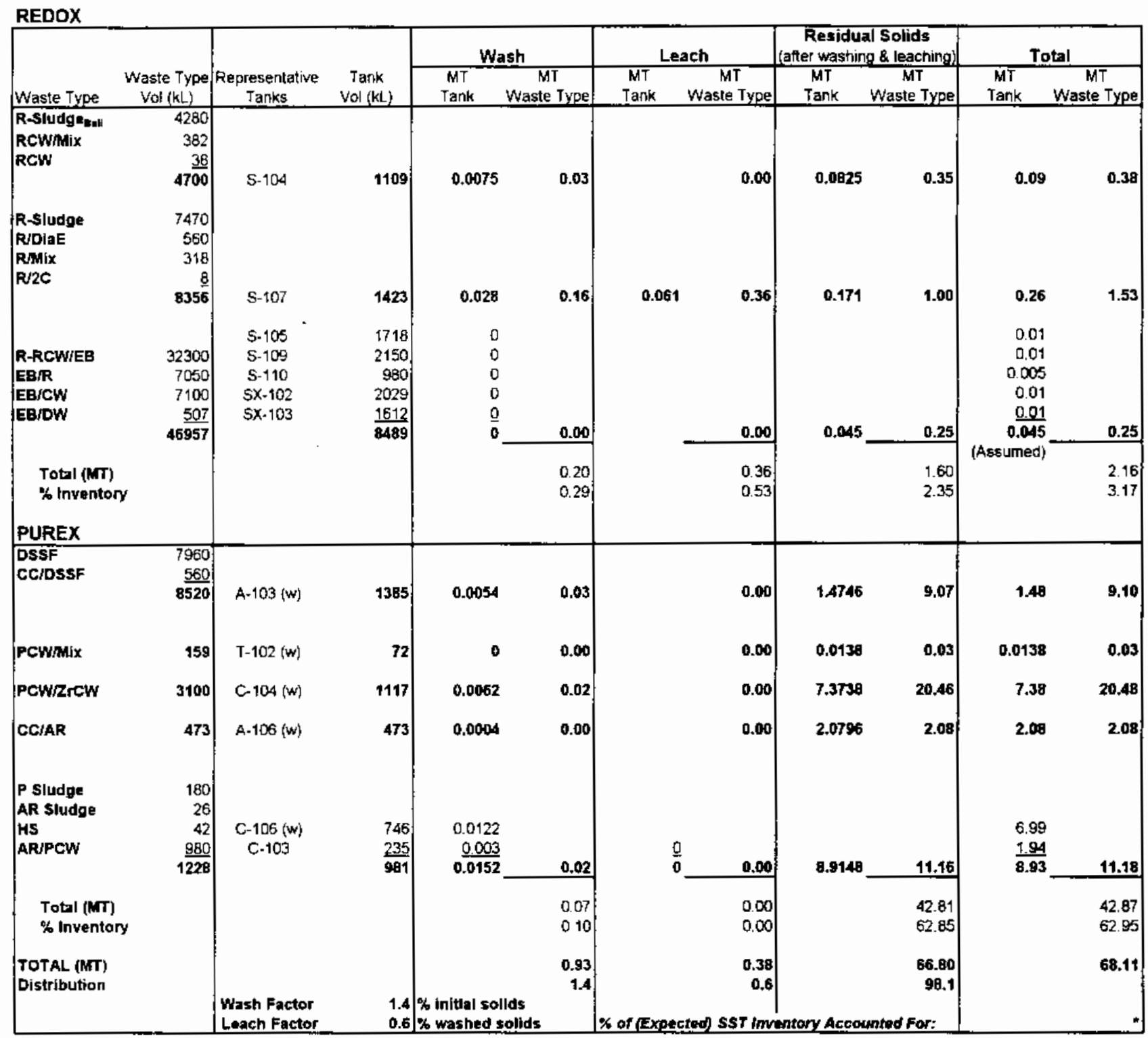

*Mg Inventory value unavaitable. 
Element: $M n$

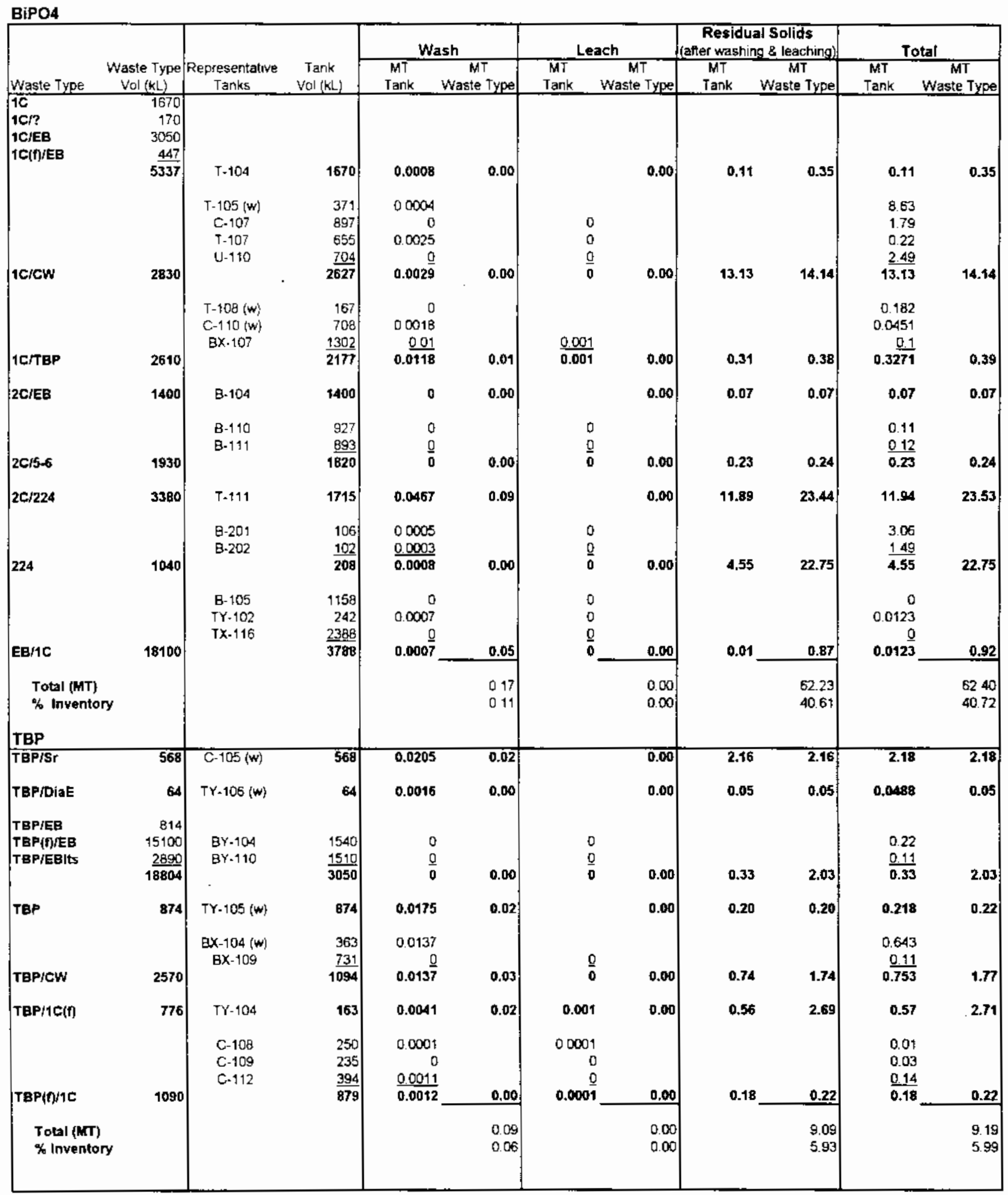


Element: Mn (Continued)

REDOX

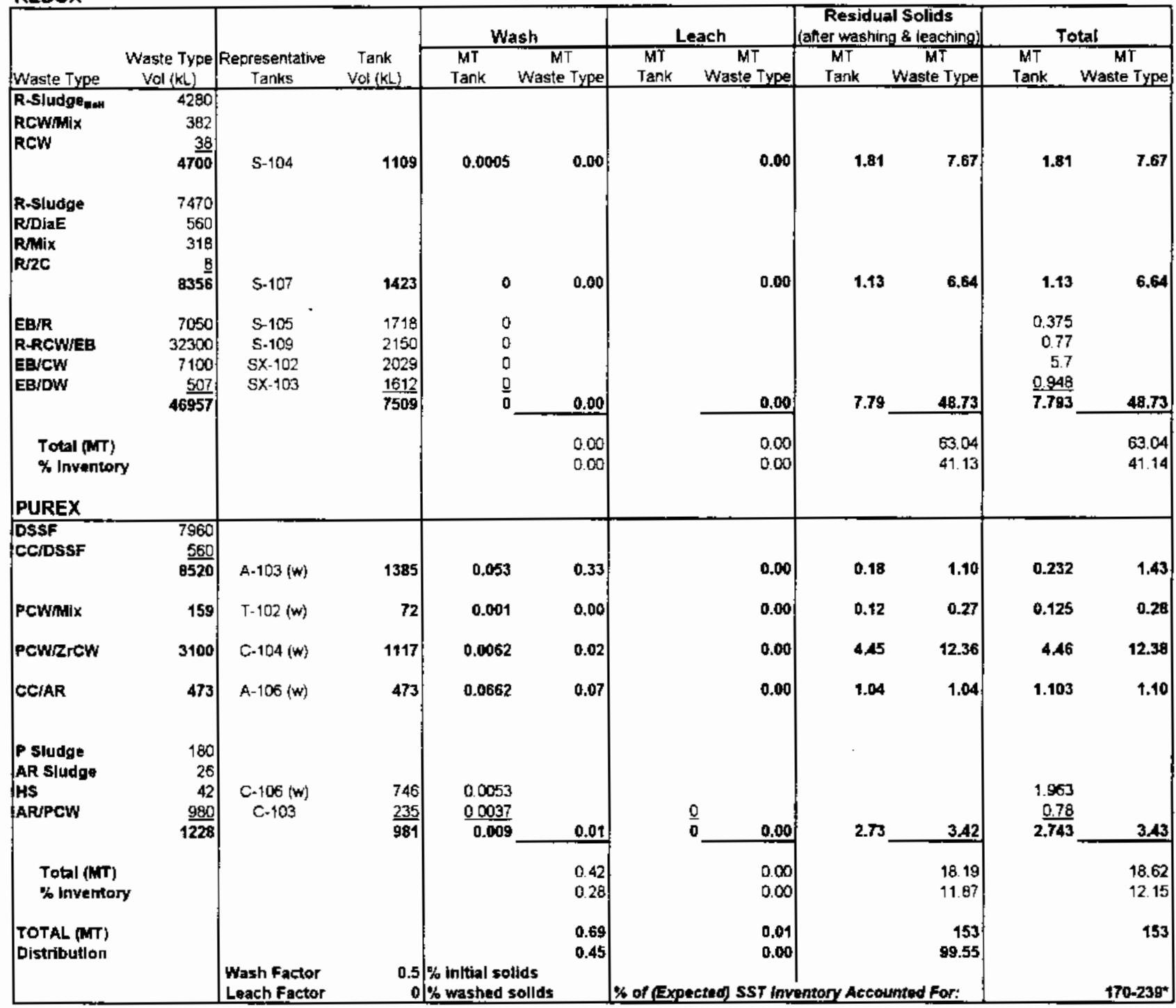

\begin{tabular}{|lc|}
\hline "Provisional" Bost-basis Total & \\
Inventory (on-going work at WHC) & $105+/-13$ \\
less TWRS DST Inventory & $-28.3{ }^{*}$ \\
Expected SST Inventory & $76.7+l-13$ \\
This Evaluation & 153 \\
& $199 \%$ \\
Expected Inventory Accounted for & \\
& \\
Range reflects 36. 7 - 50 MT Mn estimated from REDOX \\
Process. Potentially less than 28.3 MT Mn in DSTs.
\end{tabular}


Element: $\mathrm{Na}$

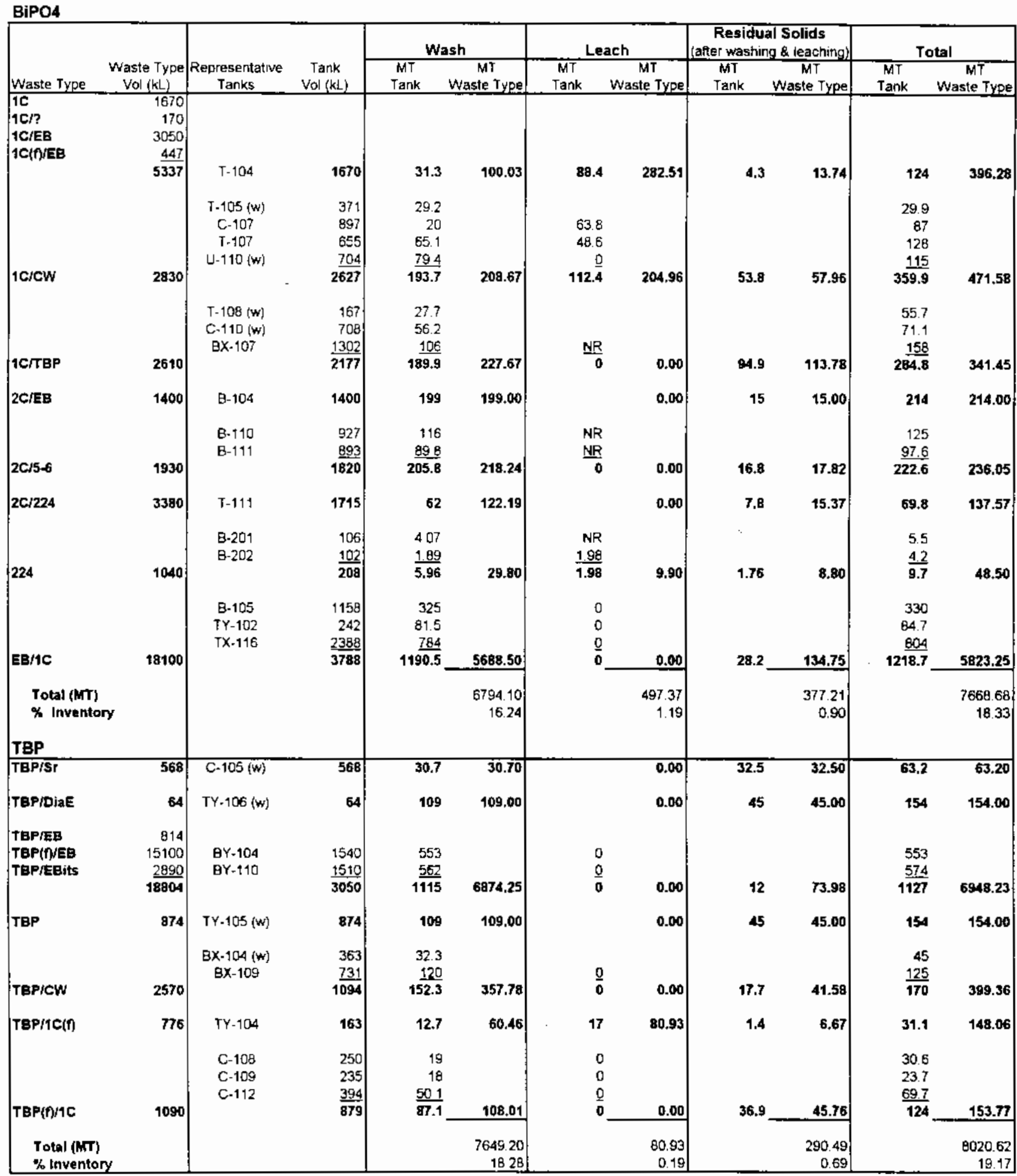


Element: $\mathrm{Na}$ (Continued)

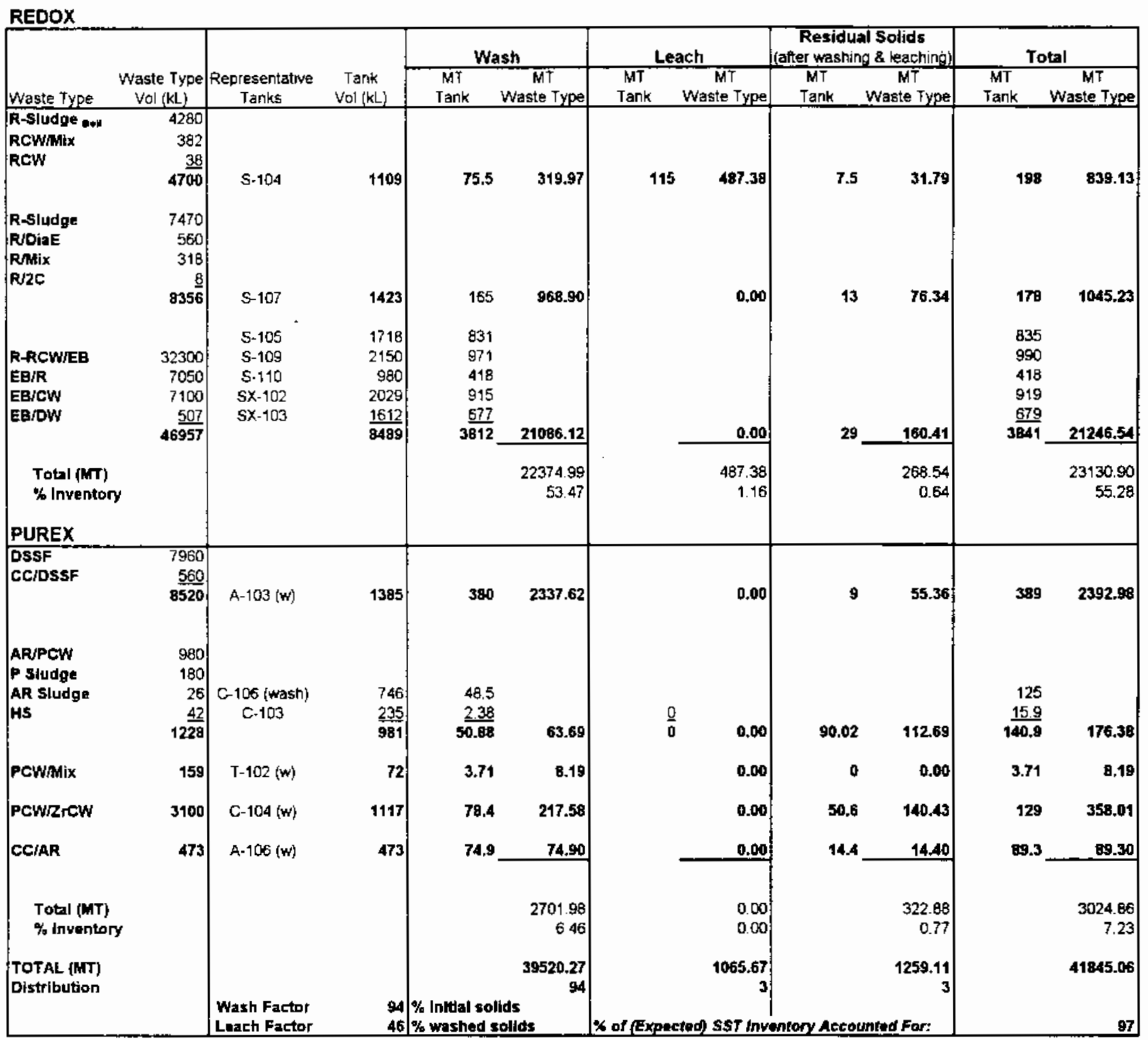

Leach factor from tests performed w/th ARPCW (Purex) wasto (C-103) used for DSSF, CC/OSSF, PCWhlix, PCW/ZrCW, and CC/AR (Purnx) waxtes.

\begin{tabular}{|lr|}
\hline Provisional" Best-basis Total & (MT) \\
Inventory (On-going work at WHC) & 54200 \\
less TWRS DSI Inventory & -11200 \\
& 43000 \\
Expected SsT Inventory & 41800 \\
This Evaluation & $87 \%$ \\
Expected Inventory Accounted for & \\
\hline
\end{tabular}


Element: Ni

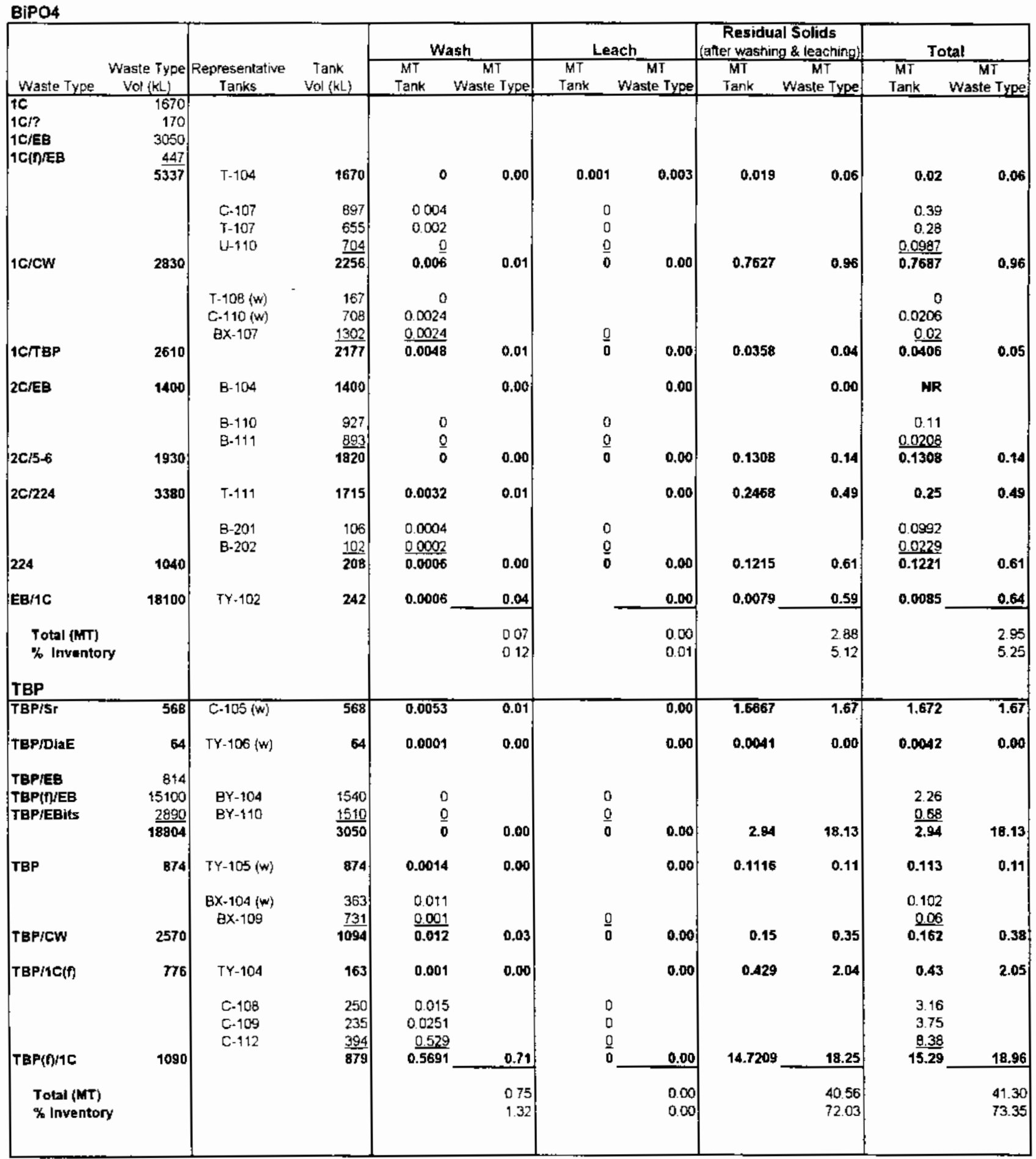


Element: Ni (Continued)

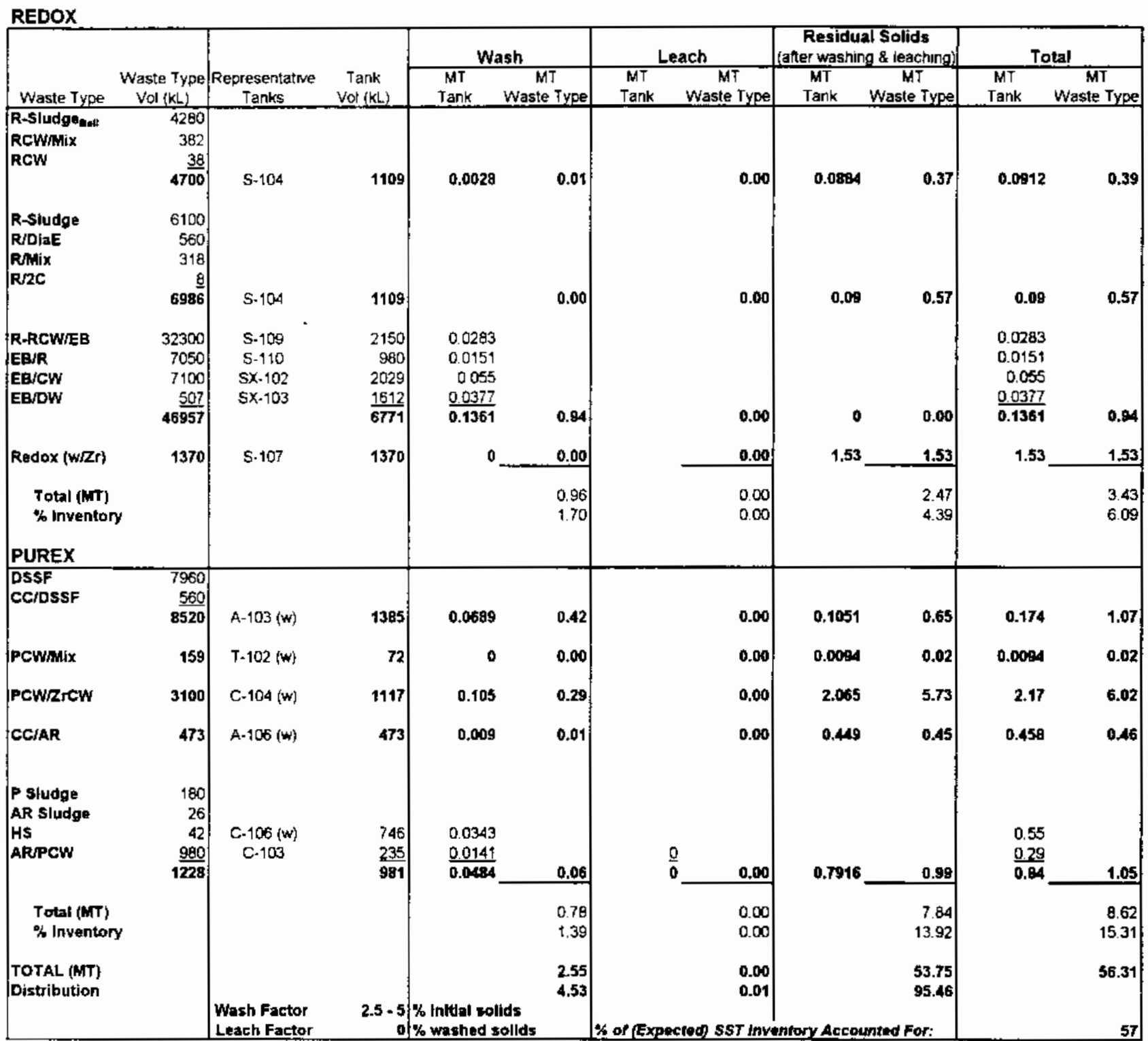

\begin{tabular}{|lc|}
\hline "Provlsionat" Best-basis Total & \\
Inventory (on-going work at WHC) & 110 \\
less TWRS DST Inventory & -10.9 \\
& \\
& $\$ 9.1$ \\
Expected \$\$T inventory & $\$ 6.3$ Refer notes \\
Exis Evaluation & $\$ 7 \%$ \\
\hline
\end{tabular}

1. Sludges are fused for analysis in nickel eruclbles; thentore, tonk-apecinc Ni bnventorles are basted on recults reportad for acid diseolution. As a result, tank-specific Inventories above may be incomplets.

2. Ho Ni analyses wefe performed on residual solids for RACW/EB mastes (Schulz 1980): therefore, this wasto type may contaln more Ni than reported above.

3. TWRS assumes -10 MT Ni in double-shall tanks, while LANL extimatec 38 MT Ni in DSTs. Using the LANL figure tor DSTs, the \% accounted for value becomes $79-124 \%$.

4. Also note that in addtlon to wastes that were scavenged wth nlckel ferrocyanide, nickel conctertraptlons appear high in $\mathrm{Zreladding} \mathrm{wastas.}$ 
Element: PO,

BiPO4

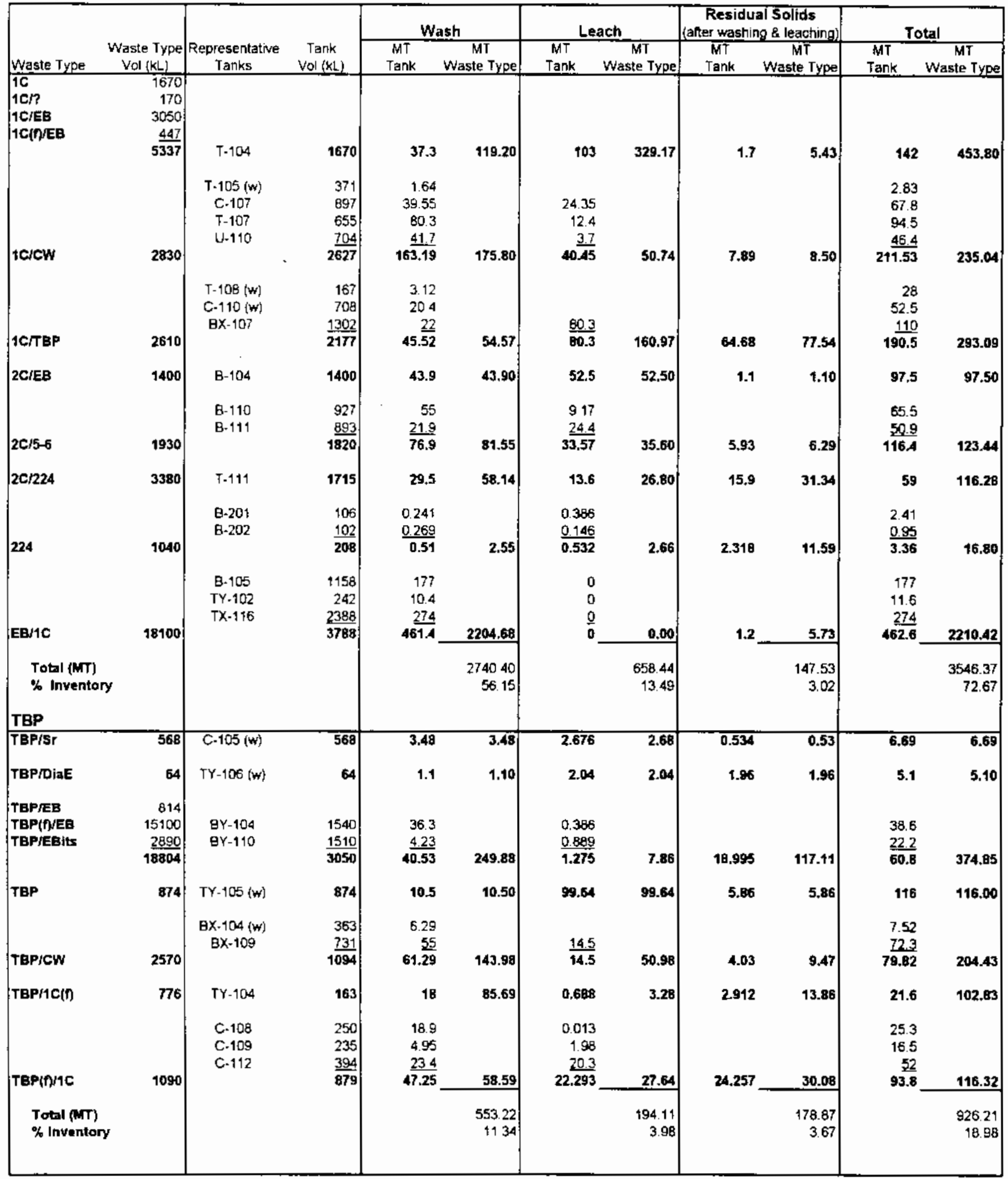


Element: $\mathrm{PO}_{4}$ (Continued)

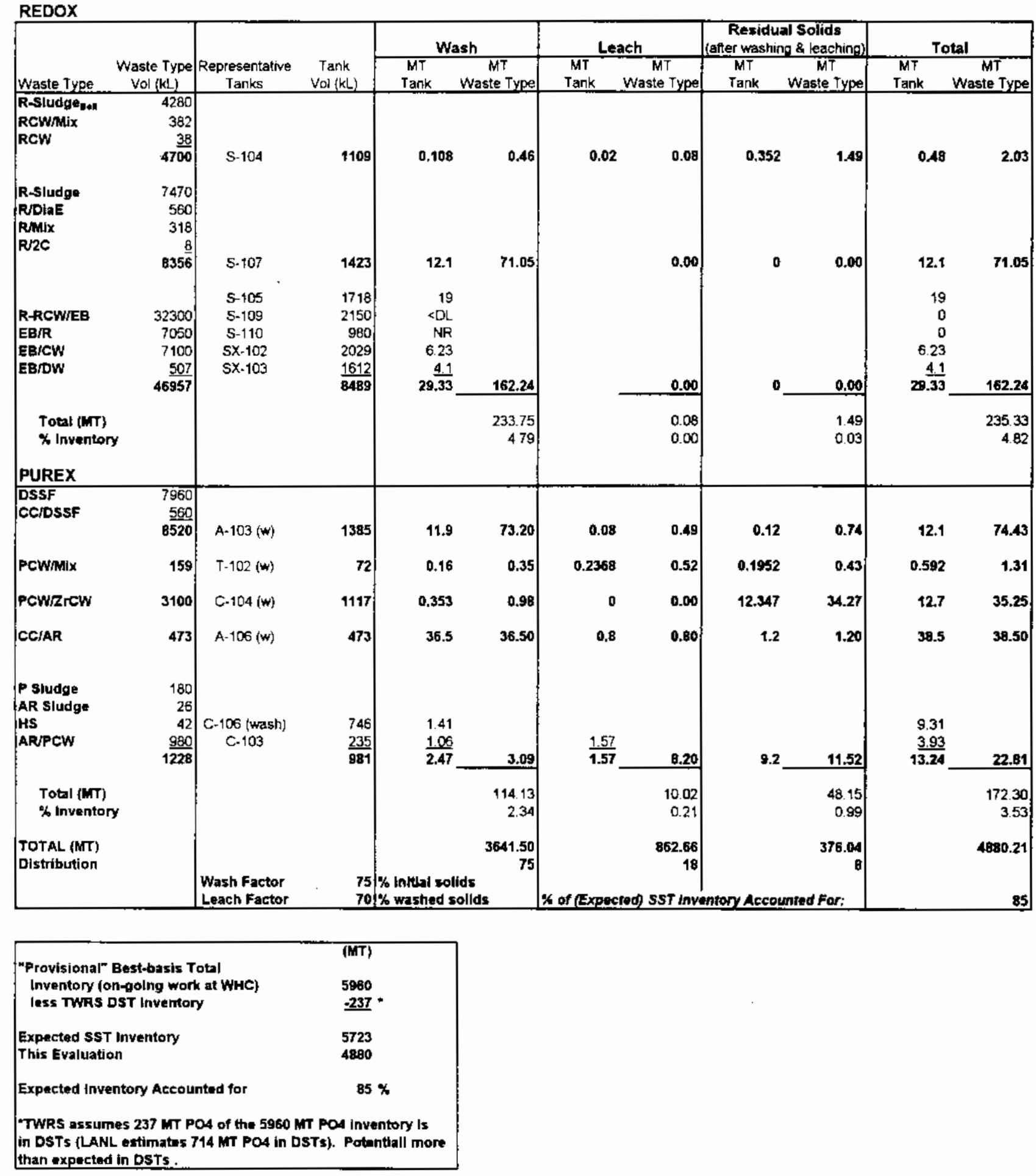


Element: Si

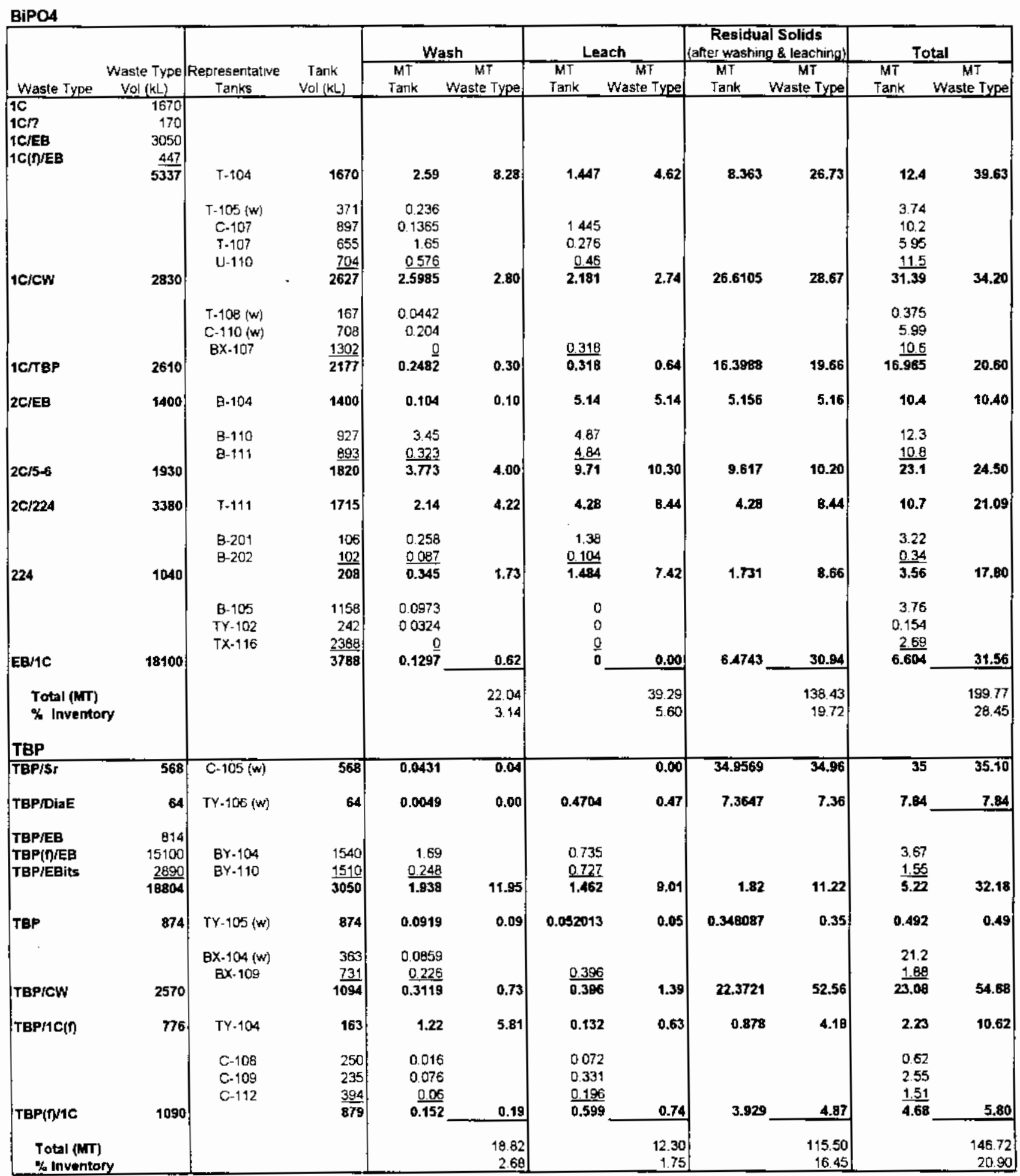


Element: Si (Continued)

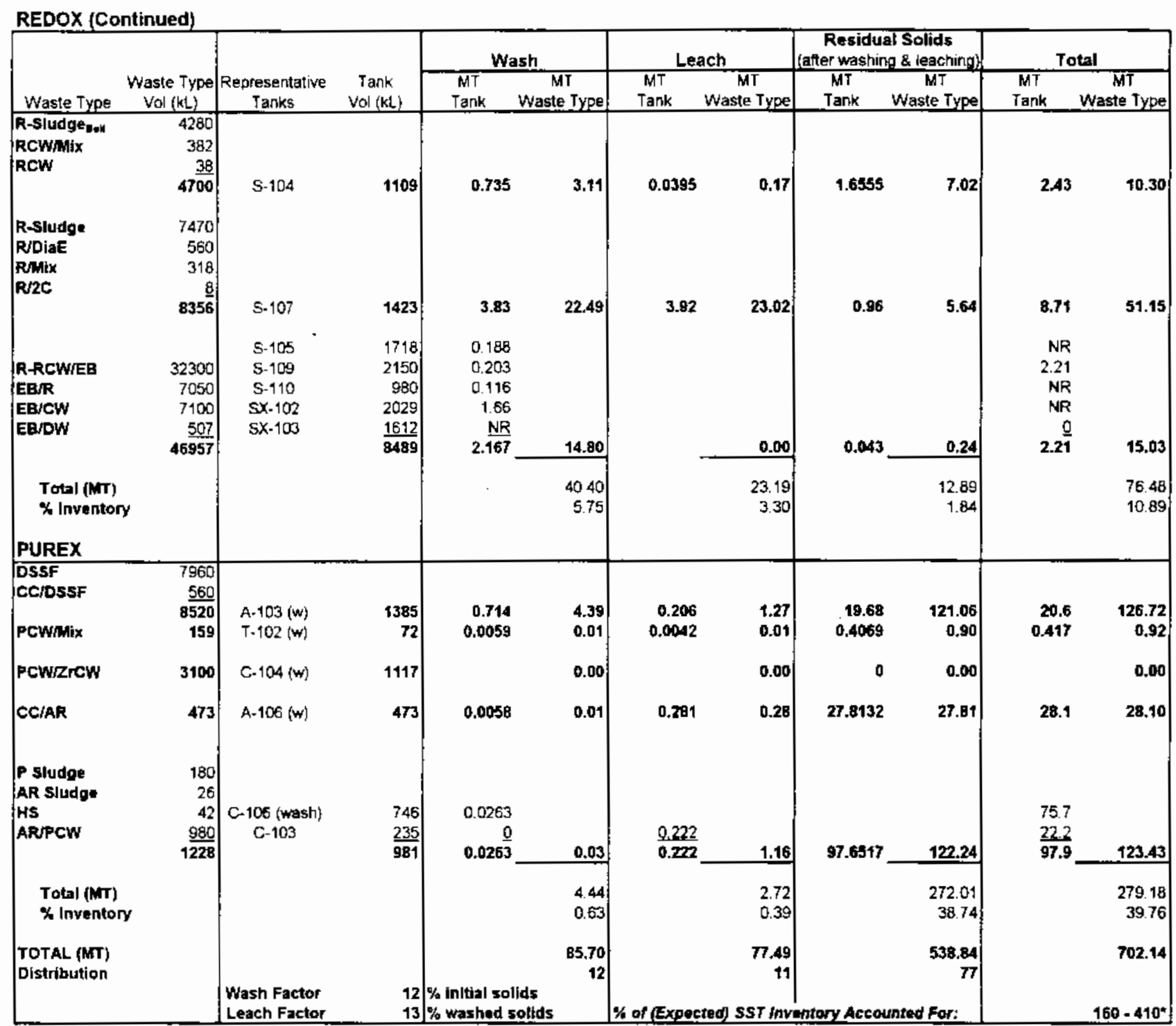

Woth that Si concentrations are very hlgh in PUREX wastes.

\begin{tabular}{|ll|}
\hline -Provisional" Best-basis Total & (MT) \\
Inventory (on-golng work at WHC) & $540+1-135$ \\
less TWRS DST Inventory & $\underline{-234}$ \\
& 306 \\
Expected SST Inventory & 702 \\
This Evaluation & $229 \%$ \\
\hline
\end{tabular}


Element: Sr

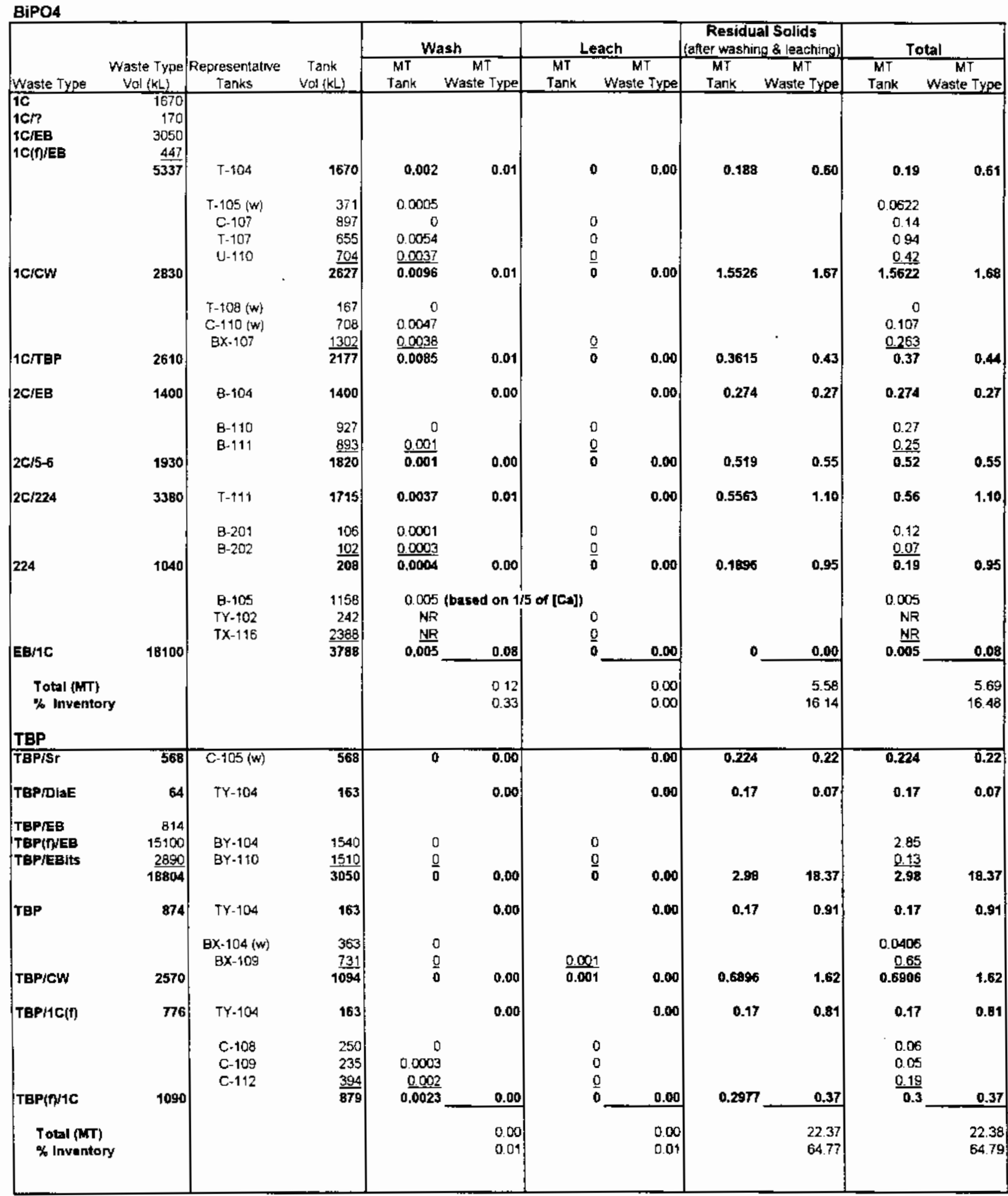


Element: Sr (Continued)

REDOX

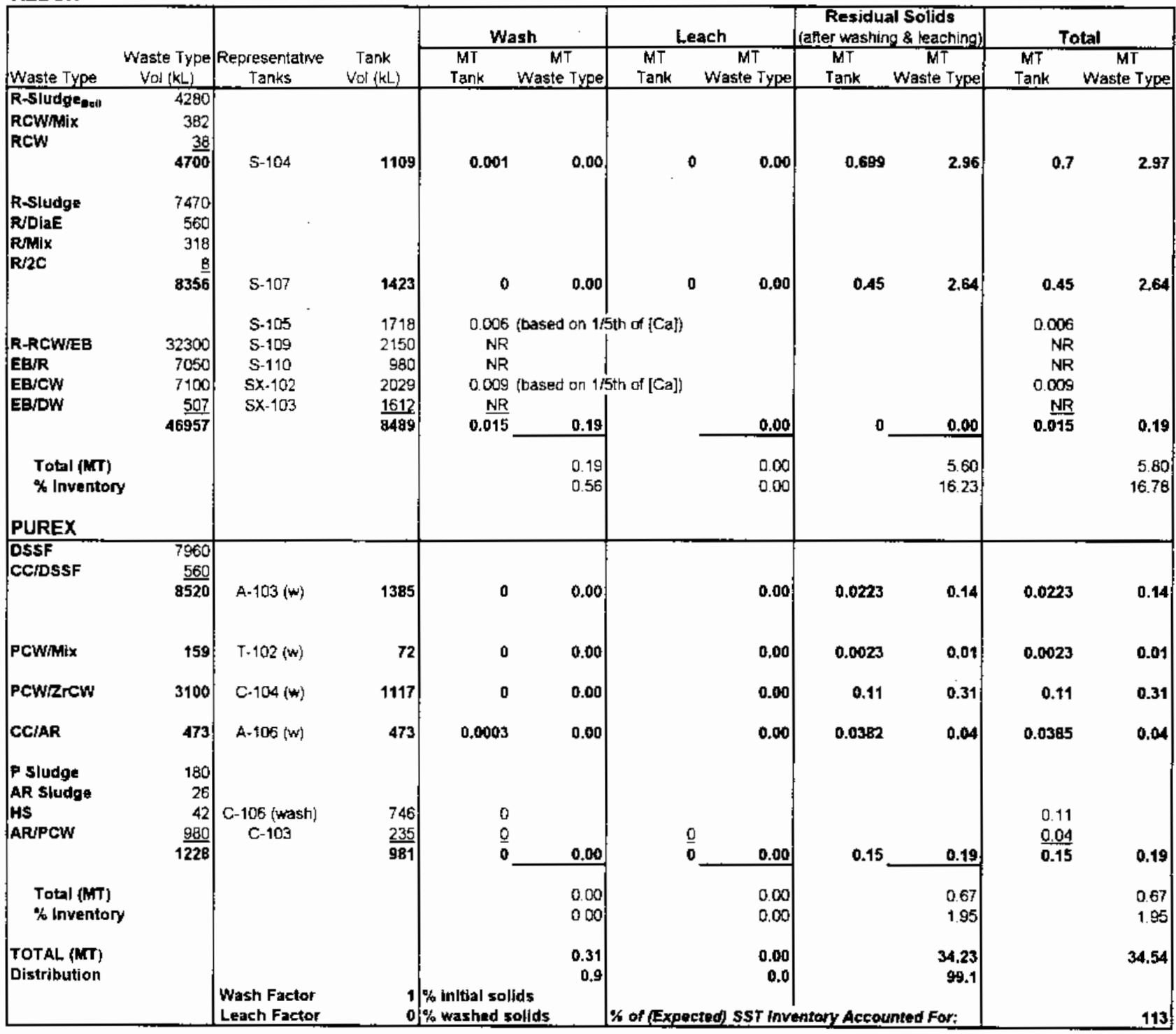

\begin{tabular}{|lc|}
\hline "Provisional" Best-basis Total & (MT) \\
Inventory (on-going work at WHC) & $\mathbf{3 1 . 3}$ \\
less TWRS DST Inventory & $-\mathbf{- 0 . 6 2}$ \\
Expected SST Inventory & $\mathbf{3 0 . 7}$ \\
This Evaluation & $\mathbf{3 4 . 5}$ \\
Expected Inventory Accounted for & $113 \%$ \\
\hline
\end{tabular}


Element: ${ }^{90} \mathrm{Sr}$

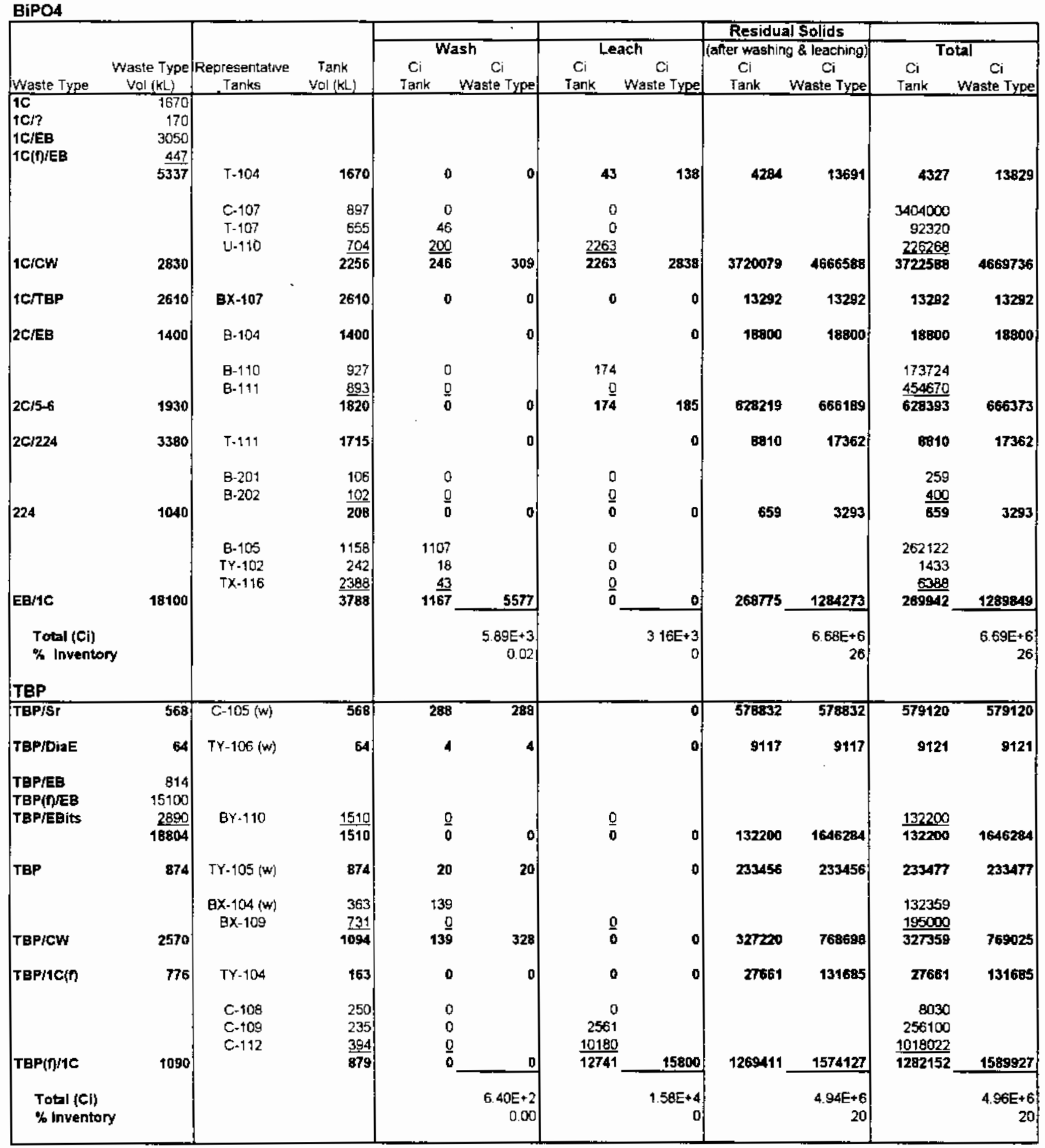


Element: ${ }^{90} \mathrm{Sr}$ (Continued)

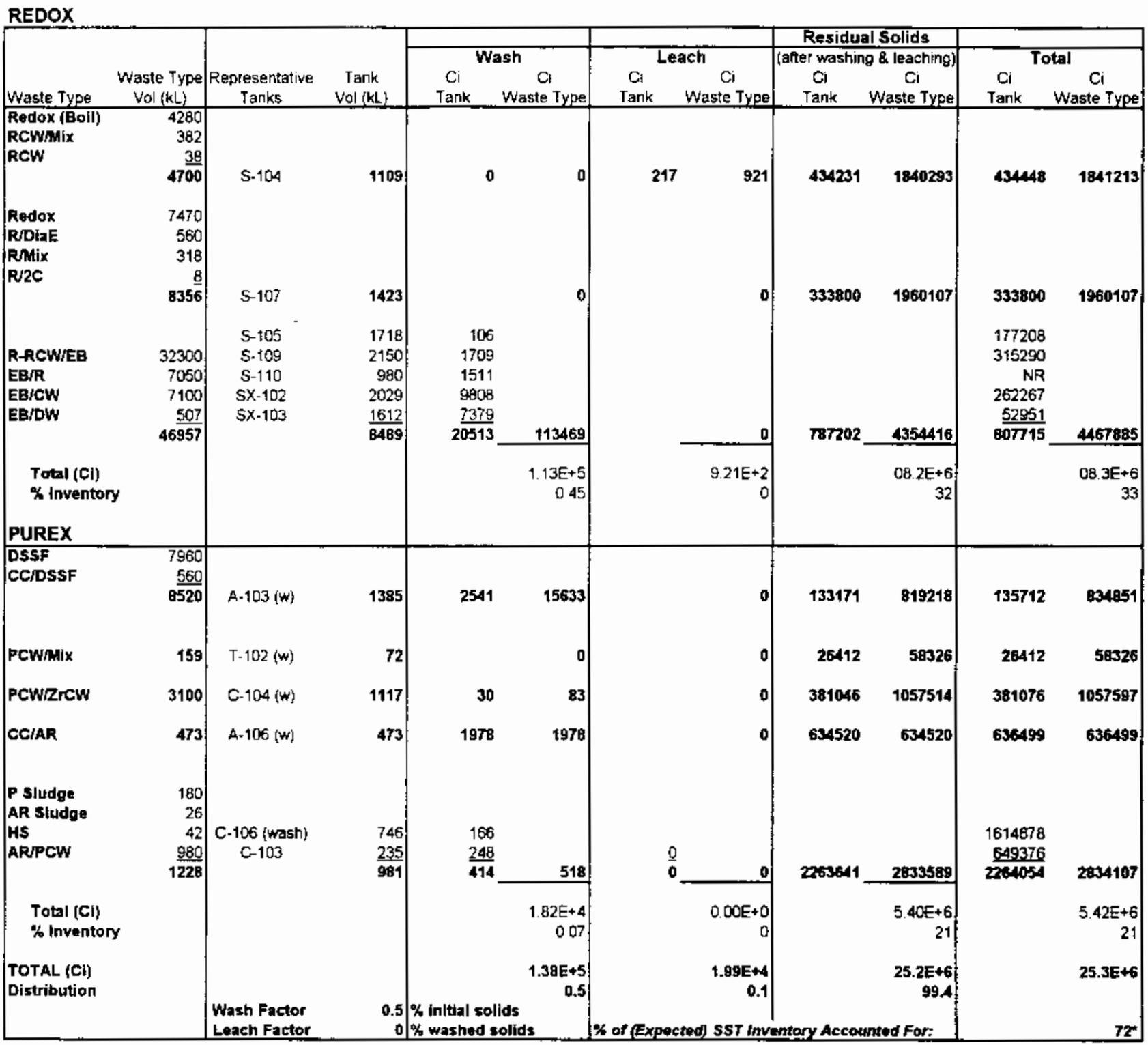

"Bosed an decaying inventories to 1999. Note that decay calculations were based on fample reporting dates, and as a result inventorles are approximations.

\begin{tabular}{|lc|}
\hline "Provisional" Best-basis Total & (Ci) \\
Inventory (on-going work at WHC) & $46.6 E+6$ \\
Iess TWRS DST Inventory & $\underline{-11,4 E+6}$ \\
& \\
Expected SST Inventory & $35.2 E+6$ \\
Thls Evaluation & $25.3 E+6$ \\
Expected Inventory Accounted for & $72 \%$ \\
\hline
\end{tabular}


Element: $U$

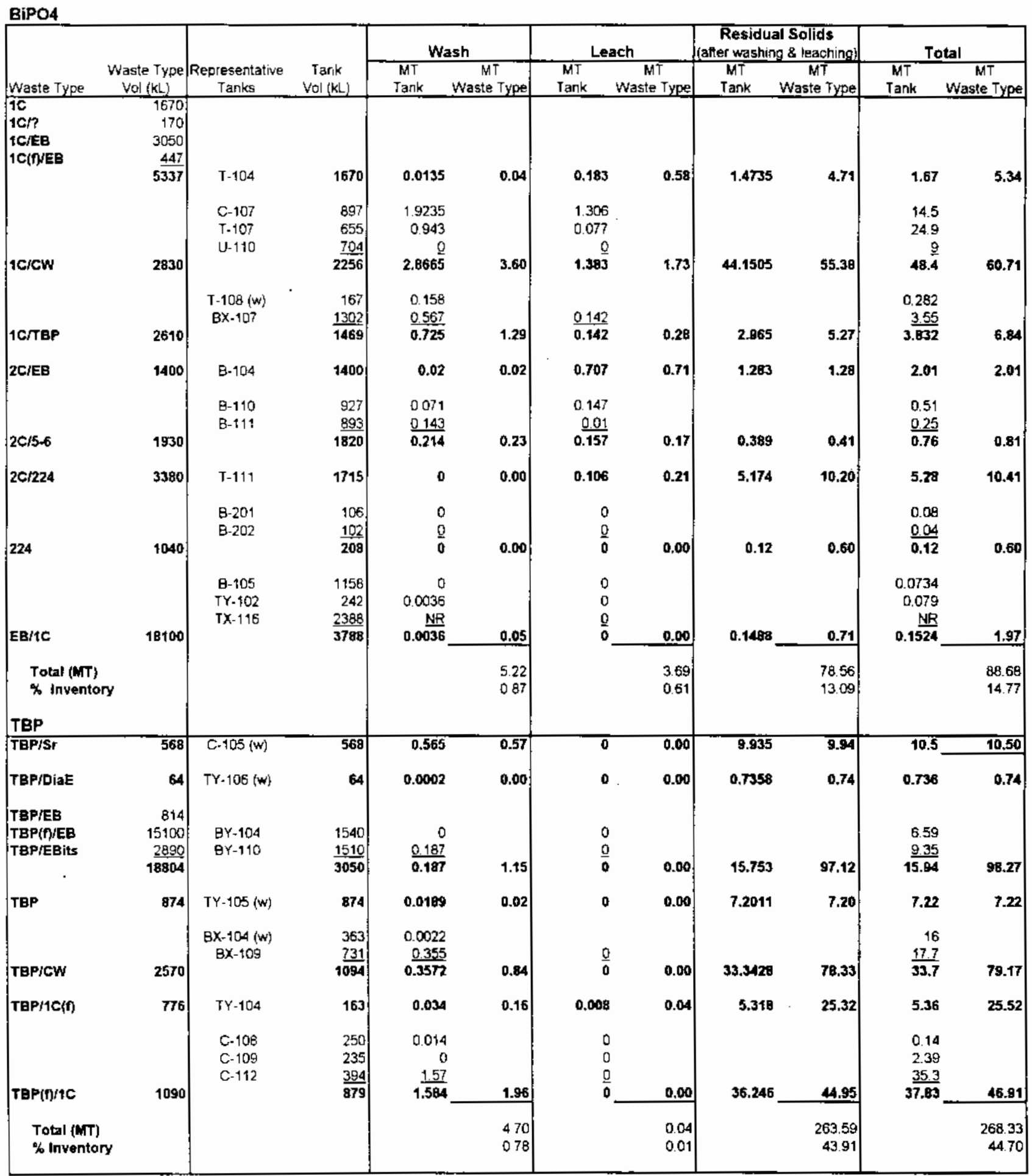


Element: $U$ (Continued)

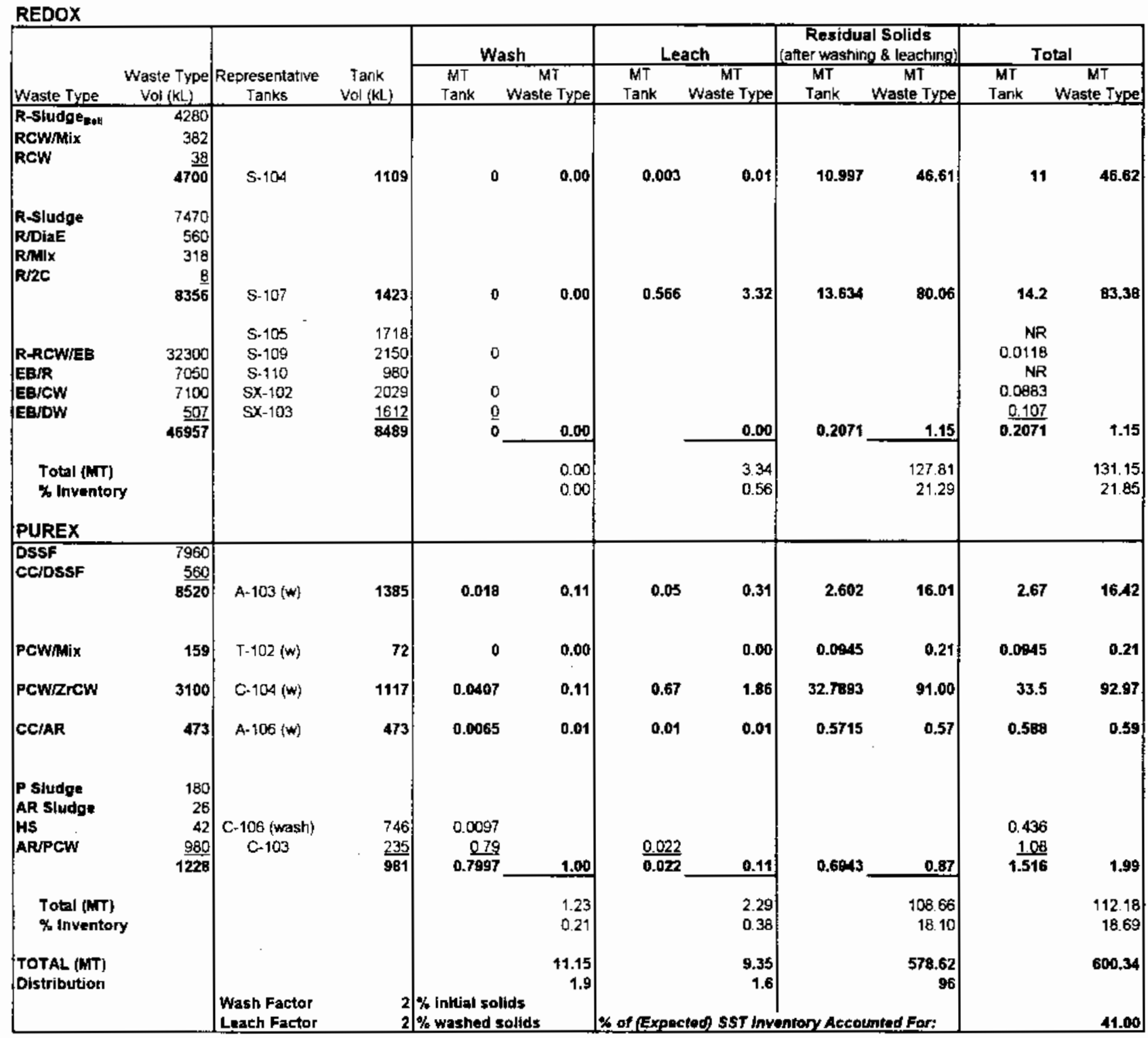

TWRS extmates $U$ inventory to be $1460 \mathrm{MT}$. The $600 \mathrm{MT} U$ determined by this anabyis suggests that:

1) retriaval of MW from the Uranium Recovery Process was vory successtut ;

2) there may be large concentratlons of $U$ in a few select tonks (e.g., C-112) that do not appar in this analysis; andior 3) there may be mw heels remaining in the bink.

\begin{tabular}{|lc|}
\hline & (MT) \\
TWRS Inventory & 1460 \\
less TWRS DST Inventory & $-\mathbf{4 . 4 9}$ \\
Expected SST Inventory & 1456 \\
This Evaluation & 600 \\
Expected Inventory Accounted for & $41 \%$ \\
\hline
\end{tabular}


Element: $\mathbf{Z r}$

BiPO4

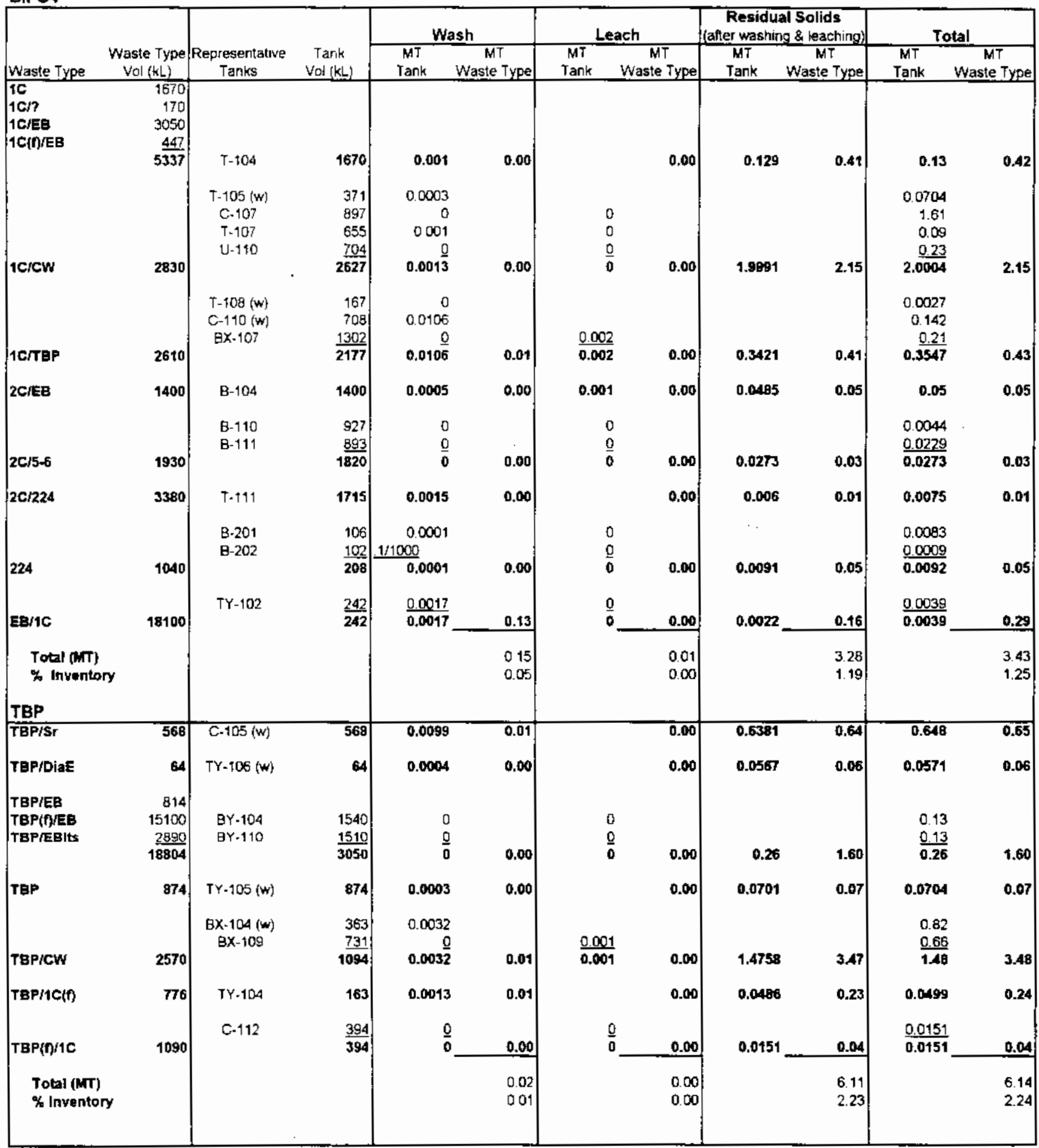


Element: $\operatorname{Zr}$ (Continued)

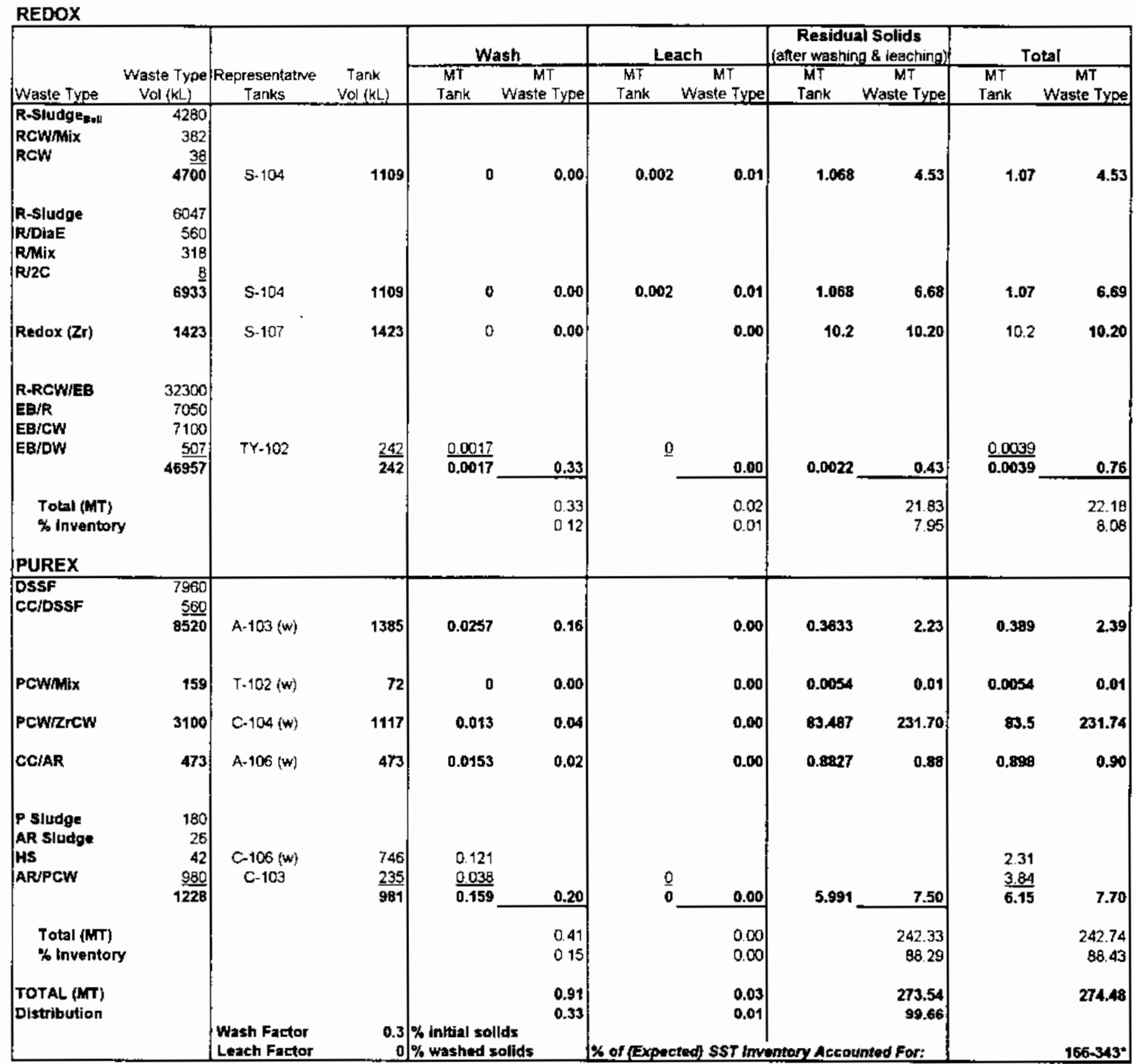

" Range reflects $45 \mathrm{MT}$ uncertainty in provisional inventory estlmate. Note that there may be potentially less than 305 mT Zr expocted in DSTs.

\begin{tabular}{|lc|}
\hline "Provisional" Best-basis Tatal & (MT) \\
Inventory (on-going work at WHC) & $430+/-45$ \\
Iess TWRS DST Inventory & $\underline{-305}$ \\
& $125.0+/-45$ \\
Expected SST Inventory & 274,0 \\
This Evaluation & $219 \%$ \\
\hline
\end{tabular}




\subsection{Conclusions and Recommendations}

The overall wash and leach factors developed by this task incorporate dissolution data from tests performed with tank wastes, which, in actuality, are mixtures of insoluble and soluble materials. As a result, these overall factors apply to a composite SST inventory and provide a technical basis for partitioning assumptions that previously were based on judgment and limited data. Table 4 compares the total residual solids for SST analytes that result from applying 1) 1995 partitioning factors based on past assumptions, and 2) new partitioning factors based on the methodology developed in this task. This table also includes a comparative ratio (new: 1995) that shows, for a particular analyte, whether residual solids (solids remaining after washing and leaching) have increased or decreased as a result of the new factors.

With the exception of chromium, analyte partitioning assumptions used in the past for planning purposes tend to be supported by the newly evaluated wash and leach factors. Because of its important role in HLW glass production, the potential two-fold increase in chromium in solids destined for disposal in HLW glass is cause for concem. The potential increase is based on the newly derived leach factor, which is dramatically less than the 60 percent factor used in past planning activities. An additional cause for concern is that the total chromium inventory may be twice as large as originally estimated [ongoing work at Westinghouse Hanford Company (WHC) to estimate best-basis total analyte inventory values].

Based on the detailed washing and leaching evaluation provided in Section 3.0, the following conclusions and recommendations are made concerning chromium:

Relatively little chromium was leached from BY-104 and BY-110 wastes. Analysis of BY-110 residual solids indicated $\mathrm{CrOOH}$, i.e., chromium (III), was present (Lumetta 1996). Assuming the chromium in these particular wastes came from evaporator bottoms, discovering what caused the reduction of chromium (VI) in alkaline supernatants sent to the evaporators may help determine if other evaporator bottom-type wastes will exhibit similar chromium leaching behavior.

- A substantial portion of the chromium inventory appeared in Redox/Evaporator Bottom salt-type wastes. Because of the poor leaching behavior exhibited by BY-104 and BY-110 wastes, no chromium leach factors were assumed for the Redox/Evaporator Bottom wastes. Data are need to determine chromium leaching behaviors in predominantly salt-type wastes. 
- The bulk of the chromium inventory in Double-shell Slurry Feed wastes appeared in residual solids because the leach factor was derived from C-103 test results; i.e., Purex solids that precipitated in Double-shell Slurry Feed wastes were assumed to behave similarly to Purex solids precipitated in C-103 waste. Actual leach data are needed to determine chromium leaching behavior in Double-shell Slurry Feed wastes. 
Table 4. Comparison of Total Residual Solids for SST Analytes Based on 1995 and Newly Derived Partitioning Factors

\begin{tabular}{|c|c|c|c|c|c|c|c|c|c|c|c|c|c|}
\hline & \multirow{3}{*}{$\begin{array}{c}\text { Current } \\
\text { TWRS } \\
\text { SST Inv } \\
\text { (MT) }\end{array}$} & \multicolumn{7}{|c|}{1995 Partitioning Factors } & \multicolumn{4}{|c|}{ New Partitioning Factors } & \multirow{3}{*}{$\begin{array}{c}\text { Comparative } \\
\text { Ratio } \\
\text { New: } 1995\end{array}$} \\
\hline & & \multicolumn{2}{|c|}{ Assumed Partitioning } & \multicolumn{2}{|c|}{ Wash Faclor } & \multirow{2}{*}{$\begin{array}{l}\text { Washed } \\
\text { Solids } \\
\text { (MT) }\end{array}$} & \multirow{2}{*}{$\begin{array}{l}\text { Eeach } \\
\text { Factor } \\
(\%)\end{array}$} & \multirow{2}{*}{$\begin{array}{l}\text { Residual } \\
\text { Sollds } \\
\text { (MT) }\end{array}$} & \multirow{2}{*}{$\begin{array}{l}\text { Wash } \\
\text { Factor } \\
(\%) \\
\end{array}$} & \multirow{2}{*}{$\begin{array}{l}\text { Washed } \\
\text { Solids } \\
\text { (MI) }\end{array}$} & \multirow{2}{*}{$\begin{array}{l}\text { Leach } \\
\text { Factor } \\
\text { (\%) }\end{array}$} & \multirow{2}{*}{$\begin{array}{l}\text { Residual } \\
\text { Sollds } \\
\text { (MT) }\end{array}$} & \\
\hline & & $\begin{array}{l}\text { Sludge } \\
\text { (MT) }\end{array}$ & $\begin{array}{c}\text { Salt Cake } \\
\text { (MT) }\end{array}$ & $\begin{array}{c}\text { Sludige } \\
(\%)\end{array}$ & $\begin{array}{c}\text { Sali Cake } \\
\text { (\%) }\end{array}$ & & & & & & & & \\
\hline $\mathrm{Ag+}$ & 0 & & & 2.0 & 99 & 0 & 0 & 0 & & & & & \\
\hline$A i+3$ & 2960 & 2220 & 739 & 8.0 & 99 & 2050 & 67 & 676 & 31 & 2345 & 60 & 938 & 0.85 \\
\hline $\mathrm{Al}+3$ (t)ed to Si) & 438 & 428 & 9.58 & 0.0 & 99 & 428 & 0 & 428 & \multicolumn{4}{|c|}{ (factors applied to combined A inventory $3398 \mathrm{MT}$ ) } & \\
\hline$B+3$ & 0 & & & 59 & 99 & 0 & 0 & 0 & & & & & \\
\hline $\mathrm{Ba}+2$ & 0.589 & 0.589 & & 0.2 & 99 & 0.588 & 0 & 0.588 & 0.9 & 0.584 & 0 & 0.584 & 0.99 \\
\hline$B i+3$ & 262 & 252 & 9.58 & 0.7 & 99 & 250 & 0 & 250 & 0.3 & 264 & 0.3 & 280 & 1.04 \\
\hline $\mathrm{Ca}+2$ & 128 & 122 & 6.42 & 3.0 & 99 & 118 & 0 & 118 & 4.0 & 123 & 2 & 121 & 1.02 \\
\hline$c d+2$ & 3.84 & 3.84 & & 11 & 99 & 3.42 & 0 & 3.42 & 15 & 3 & 0 & 3 & 0.96 \\
\hline $\mathrm{Co}+3$ & 0 & & & 13 & 99 & 0 & 0 & 0 & & & & & \\
\hline \multirow[t]{2}{*}{$\mathrm{Cr}+3 /+6$} & 268 & 121 & 147 & 18 & 99 & 101 & 60 & 40.3 & 59 & 110 & 14 & 94 & $2.34^{(a)}$ \\
\hline & & & & & & & & & $61^{(b)}$ & 105 & $10^{(\mathrm{b})}$ & 94 & $2.34^{(\mathrm{a})}$ \\
\hline $\mathrm{Cu}+2$ & 812 & 26.2 & $7 B 6$ & 3.7 & 99 & 33.1 & 0 & 33.1 & & & & & \\
\hline $\mathrm{Fe}+3$ & 631 & 607 & 23.7 & 0.67 & 99 & 603 & 0 & 603 & 0.4 & 628 & 0.2 & 627 & 1.04 \\
\hline $\mathrm{K}+$ & 55.3 & 2.43 & 52.9 & 64 & 99 & 1.40 & 0 & 1.40 & & & & & \\
\hline $\mathrm{La}+3$ & 28 & 28 & & 0.24 & 99 & 27.9 & 0 & 27.9 & & & & & \\
\hline $\mathrm{Mg}+2$ & 0 & & & 1.7 & 99 & 0 & 0 & 0 & 1 & 0 & 0.6 & 0 & \\
\hline$M n+4$ & 120 & 118 & 2.41 & 0.92 & 99 & 117 & 0 & 117 & 0.5 & 120 & 0 & 120 & 1.02 \\
\hline $\mathrm{Na}+$ & 57342 & 542 & 56800 & 96 & 99 & 590 & 25 & 442 & 94 & 3462 & 46 & 1870 & $2.07^{(\mathrm{c})}$ \\
\hline \multirow[t]{2}{*}{$\mathrm{Na}+($ W/AVSI) } & 460 & 460 & 0 & 0 & 99 & 460 & 0 & 460 & $95^{(t)}$ & 2885 & $45^{(b)}$ & 1587 & $1.76^{(t)}$ \\
\hline & & & & & & & & & \multicolumn{4}{|c|}{ (factors applied to combined $\mathrm{Na}$ invertory $57707 \mathrm{MT}$ ) } & \\
\hline $\mathrm{Ni}+2$ & 203 & 199 & 4.06 & 2.2 & 99 & 195 & 0 & 195 & 2.5 & 198 & 0 & 198 & 1.02 \\
\hline $\mathrm{Pb}+2$ & 28.3 & 25 & 3.29 & 6.8 & 99 & 23.3 & 0 & 23.3 & & & & & \\
\hline PO4(-3) & 4730 & 1830 & 2900 & 40 & 99 & 1127 & 74 & 293 & 75 & 1183 & 70 & 355 & 1.21 \\
\hline$S i+4$ & 14.5 & 14.1 & 0.406 & 2.5 & 99 & 13.8 & 0 & 13.8 & 12 & 404 & 13 & 351 & 0.77 \\
\hline Si+4 (tad ban) & 444 & 444 & 0 & 0 & 99 & 444 & 0 & 444 & \multicolumn{4}{|c|}{ (factors applied to combined Si inventory $459 \mathrm{MT}$ ) } & \\
\hline $\mathrm{Sr}+2$ & 36.1 & 35.7 & 0.36 & 0.62 & 99 & 35.5 & 0 & 35.5 & 1.0 & 36 & 0 & 36 & 1.01 \\
\hline$u+6$ & 1407 & 1322 & 85.2 & 2.1 & 99 & 1295 & 0 & 1295 & 2.0 & 1379 & 2 & 1352 & 1.04 \\
\hline $\mathrm{Zn+2}$ & 0 & & & 3.2 & 99 & 0 & 0 & 0 & & & & & \\
\hline$Z r+4$ & 382 & 378 & 3.82 & 0.26 & 99 & 377 & $D$ & 377 & 0.3 & 381 & 0 & 381 & 1.01 \\
\hline
\end{tabular}

(a) This ratio is based on the Cr SST inventory estimaled as of 1995. Ongoing work at WHC indicates this inventory may be twice as farge as originally estimated. (b) Factors presented in June 30, 1996 drafl report.

(c) This ratio is based on the Na SST inventory estimated as of 1995. Ongoing work al WHC indicates this inventory is smaller than originally estimaled.

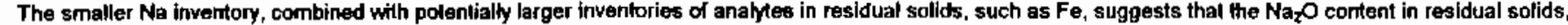
on an oxide basis, may be similar to the $14 \%$ used in past planning activities. 


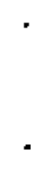




\subsection{References}

Baldwin, J. H., L. C. Amato, B. J. Morris. 1996. Tank Characterization Report for SingleShell Tank 241-T-108, WHC-SD-WM-ER-554, Rev. 0, Westinghouse Hanford Company, Richland, Washington.

Boomer, K. D., et al. 1993. Tank Waste Technical Options Report, Appendix G. WHCEP-06110, Rev. 0, Westinghouse Hanford Company, Richland, Washington.

Brown, T. M. and L. Jensen. 1993. Tank Characterization Report for Single-Shell Tank 241-U-110, WHC-EP-0643, Rev. 1, Westinghouse Hanford Company, Richland, Washington.

Colton, N. G. 1995. Sludge Pretreatment Chemistry Evaluation: Enhanced Sludge Washing Separation Factors, PNL-10512, Pacific Northwest National Laboratory, Richland, Washington.

Field, J. G., J. Jo, and T. T. Tran. 1996a. Tank Characterizarion Report for Single-Shell Tank 241-B-104, WHC-SD-WM-ER-552, Rev. 0, Westinghouse Hanford Company, Richland, Washington.

Field, J. G., R. D. Schreiber, and B. C. Simpson. 1996b. Tank Characterization Report for Single-Shell Tank 241-BX-109, WHC-SD-WM-ER-572, Rev. 0, Westinghouse Hanford Company, Richland, Washington.

Giamberardini, K. K. 1993. PNL 325 Laboratory Single-Shell Tank Waste Characterization, Tank B-111, Cores 29 and 30, WHC-SD-WM-DP-041, Westinghouse Hanford Company, Richland, Washington.

Hanlon, B. M. 1996. Waste Tank Summary Report for Month Ending November 30, 1995, WHC-EP-0182-92, Westinghouse Hanford Company, Richland, Washington.

Hill, J. G., G. S. Anderson, and B. C. Simpson. 1995. The Sort on Radioactive Waste Type Model: A Method to Sort Single-Shell Tanks into Characteristic Groups, PNL9814 Rev 2, Pacific Northwest National Laboratory, Richland, Washington. 
Kocher, K. L. 1993. Single-Shell Tank Waste Characterization Tank 241-S-104 Data Package, "222-S Laboratory Single-Shell Tank Waste Characterization, Tank S-104 Cores 42, 43 and 44 Validation Summary," WHC-SD-WM-DP-031, Addendum 2 Rev. 0, Westinghouse Hanford Company, Richland, Washington.

Kristofski, J. G. 1993. Single-Shell Tank Waste Characterization 241-BX-107 Cores 40 and 41, "Sample Data Summary," WHC-SD-WM-DP-028, Addendum 2, Rev. 0, Westinghouse Hanford Company, Richland, Washington.

Lumetta, G. J. and B. M. Rapko. 1994. Washing and Alkaline Leaching of Hanford Tank Sludges: A Status Report, PNL-10078, Pacific Northwest National Laboratory, Richland, Washington.

Lumetta, G. J., B. M. Rapko, M. J. Wagner, and J. Liu. 1996. Washing and Caustic Leaching of Hanford Tank Sludges: Results of FY 1996 Studies, PNNL-11278, Rev. 1, Pacific Northwest National Laboratory, Richland, Washington.

Orme, R. M. 1995. TWRS Process Flowsheet, WHC-SD-WM-TI-613, Rev. 1, Westinghouse Hanford Company, Richland, Washington.

Rapko, B. M., G. J. Lumetta, and M. J. Wagner. 1995. Washing and Caustic Leaching of Hanford Tank Sludges: Results of FY 1995 Studies, PNL-10712, Pacific Northwest National Laboratory, Richland, Washington.

Schulz, W. W. 1980. Removal of Radionuclides from Hanford Defense Waste Solutions, RHO-SA-51, Rockwell Hanford Operations, Richland, Washington.

Simpson, B. C., G. L. Borsheim, L. Jensen. 1993a. Tank Characterization Report: Tank 24I-C-109, WHC-EP-0668, Westinghouse Hanford Company, Richland, Washington.

Simpson, B. C., G. L. Borsheim, L. Jensen. 1993b. Tank Characterization Data Report: Tank 241-C-112, WHC-EP-0640, Westinghouse Hanford Company, Richland, Washington.

Temer, D. J. and R. Villarreal. 1995 Sludge Washing and Alkaline Leaching Tests on Actual Hanford Tank Sludge: A Starus Report, LAUR 95-2070, Lost Alamos National Laboratory, Los Alamos, New Mexico. 
Temer, D. J. and R. Villarreal. 1996. Sludge Washing and Alkaline Leaching Tests on Actual Hanford Tank Sludge: FY 1996 Results, LAUR 96-2839, Los Alamos National Laboratory, Los Alamos New Mexico.

Weiss, R. L. and B. M. Mauss. 1987a. Data Transmittal Package for 241-TY-102 Waste Tank Characterization, SD-RE-TI-183, Rev. 0, Rockwell Hanford Operations, Richland, Washington.

Weiss, R. L. and B. M. Mauss. 1987b. Data Transmittal Package for 241-TY-104 Waste Tank Characterization, SD-RE-TI-182, Rev. 0, Rockwell Hanford Operations, Richland, Washington.

Weiss, R. L. and B. M. Mauss. 1987c. Data Transmittal Package for 241-TY-105 Waste Tank Characterization, SD-RE-TI-186, Rev. 0, Rockwell Hanford Operations, Richland, Washington.

Weiss, R. L. and B. M. Mauss. 1987d. Data Transmittal Package for 241-TY-106 Waste Tank Characterization, SD-RE-TI-181, Rev, 0, Rockwell Hanford Operations, Richland, Washington.

Weiss, R. L. and K. E. Schull. 1988a. Data Transmittal Package for 241-A-103 Waste Tank Characterization, SD-RE-TI-198, Rev. 0, Rockwell Hanford Operations, Richland, Washington.

Weiss, R. L. and K. E. Schull. 1988b. Data Transmittal Package for 241-A-106 Waste Tank Characterization, SD-RE-TI-200, Rev. 0, Rockwell Hanford Operations, Richland, Washington.

Weiss, R. L. and K. E. Schull. 1988c. Data Transmittal Package for 241-BX-104 Waste Tank Characterization, SD-RE-TI-206, Rev. 0, Rockwell Hanford Operations, Richland, Washington.

Weiss, R. L. and K. E. Schull. 1988d. Data Transmittal Package for 241-BX-105 Waste Tank Characterization, SD-RE-TI-202, Rev. 0, Rockwell Hanford Operations, Richland, Washington.

Weiss, R. L. and K. E. Schull. 1988e. Data Transmittal Package for 241-C-103 Waste Tank Characterization, SD-RE-TI-203, Rev. 0, Rockwell Hanford Operations, Richland, Washington. 
Weiss, R. L. and K. E. Schull. 1988f. Data Transmittal Package for 241-C-104 Waste Tank Characterization, SD-RE-TI-199, Rev. 0, Rockwell Hanford Operations, Richland, Washington.

Weiss, R. L. and K. E. Schull. 1988g. Data Transmittal Package for 24I-C-105 Waste Tank Characterization, SD-RE-TI-204, Rev. 0, Rockwell Hanford Operations, Richland, Washington.

Weiss, R. L. and K. E. Schull. 1988h. Data Transmittal Package for 24I-C-106 Waste Tank Characterization, SD-RE-TI-205, Rev. 0, Rockwell Hanford Operations, Richland, Washington.

WHC. 1993a. PNL 325 Laboratory Single-Shell Tank Waste Characterization Tank 24I-B201, Cores 26 and 27 Data Package, WHC-SD-WM-DP-037, Rev. 0, Westinghouse Hanford Company, Richland, Washington.

WHC. 1993b. Westinghouse 222-S Analytical Laboratory Single-Shell Tank Waste Characterization Tank C-110 Cores 37, 38 and 39, WHC-SD-WM-DP-027, Addendum 1, Rev. 0, Westinghouse Hanford Company, Richland, Washington.

WHC. 1993c. PNL 325 Laboratory Single-Shell Tank Waste Characterization Tank T-102, Cores 55 and 56 Validation Summary, WHC-SD-WM-DP-052, Addendum 1, Rev. 0, Westinghouse Hanford Company, Richland, Washington.

WHC. 1993d. Westinghouse 222-S Laboratory Single-Shell Tank Waste Characterization, Tank 241-T-104 Cores 45 and 46 Validation Summary, WHC-SD-WM-DP-032, Addendum 1, Rev. 0, Westinghouse Hanford Company, Richland, Washington.

WHC. 1993e. Westinghouse 222-S Laboratory Single-Shell Tank Waste Characterization, Tank T-107 Cores 50, 51, and 52 Validation Summary, WHC-SD-WM-042, Addendum 1A, Rev. 1, Westinghouse Hanford Company, Richland, Washington.

WHC. 1993f. Westinghouse 222-S Laboratory Single-Shell Tank Waste Characterization, Tank 241-T-111 Cores 31 and 33 Validation Report, WHC-SD-WM-DP-024, Addendum 1, Rev. 0, Westinghouse Hanford Company, Richland, Washington. 


\section{Appendix A}

\section{Tank Waste Data Summary Worksheets}




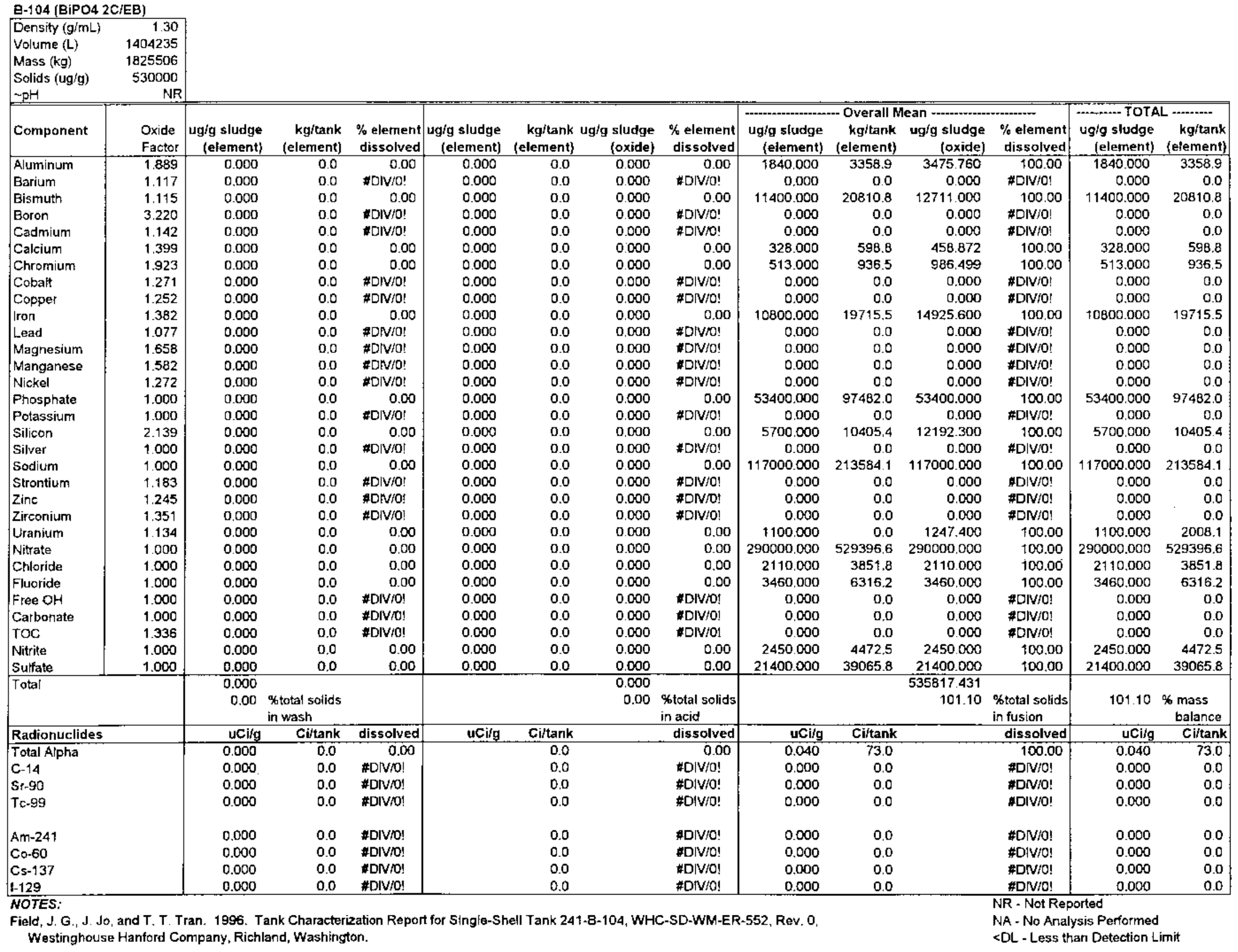


$\mathrm{B}-105$ (EB, 1C BiPO4)

\begin{tabular}{|c|c|c|c|c|c|c|c|c|c|c|c|c|c|c|}
\hline \begin{tabular}{|l|} 
Density $(\mathrm{g} / \mathrm{mL})$ \\
Volume $(\mathrm{L})$ \\
Mass $(\mathrm{kg})$ \\
Solids $(\mathrm{ug} / \mathrm{g})$ \\
$\sim \mathrm{pH}$ \\
\end{tabular} & $\begin{array}{r}1.70 \\
1006810 \\
1711577 \\
580000 \\
13.0 \\
\end{array}$ & & & & & & & & & & & & & \\
\hline Component & $\begin{array}{l}\text { Oxide } \\
\text { Factor }\end{array}$ & $\begin{array}{r}\text { ug/g sludge } \\
\text { (element) }\end{array}$ & $\begin{array}{l}\text { WATER :- } \\
\text { kg/tank } \\
\text { (element) }\end{array}$ & $\begin{array}{l}\text { \% element } \\
\text { dissolved }\end{array}$ & $\begin{array}{r}\text { ug/g sludge } \\
\text { (element) }\end{array}$ & 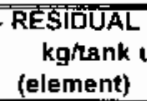 & $\begin{array}{l}\text { SOLIDS --- } \\
\text { ug/g sludge } \\
\text { (oxide) }\end{array}$ & $\begin{array}{l}\text { \% element } \\
\text { dissolved }\end{array}$ & $\begin{array}{r}\text { ug/g sludge } \\
\text { (element) }\end{array}$ & $\begin{array}{r}\text { kg/tank } \\
\text { (element) }\end{array}$ & $\begin{array}{r}g / g \text { sludge } \\
\text { (oxide) }\end{array}$ & $\begin{array}{l}\% \text { element } \\
\text { dissolved }\end{array}$ & $\begin{array}{c}\text { ug/g sludge } \\
\text { (element) }\end{array}$ & 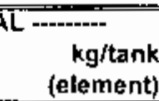 \\
\hline Aluminum & 1.889 & 252.882 & 432.8 & 23.46 & 825.000 & 1412.1 & 1558.425 & 76.54 & 0.000 & 0.0 & 0.000 & 0.00 & 1077.882 & 1844.9 \\
\hline Barium & 1.117 & 0.000 & 0.0 & \#DIV/o! & 0.000 & 0.0 & 0.000 & \#DIV/o! & 0.000 & 0.0 & 0.000 & \&DIV/ol & 0.000 & 0.0 \\
\hline Bismuth & 1.145 & 0.000 & 0.0 & \#DIV/O! & 0.000 & 0.0 & 0.000 & \#DIV/0! & 0.000 & 0.0 & 0.000 & \#DIV/O? & 0.000 & 0.0 \\
\hline Boron & 3.220 & 0.000 & 0.0 & \#DIV/O! & 0.000 & 0.0 & 0.000 & \#DIVIO! & 0.000 & 0.0 & 0.000 & $\#$ \#IV/O! & 0.000 & 0.0 \\
\hline Cadmium & 1.142 & 0.000 & 0.0 & \#DN/O! & 0.000 & 0.0 & 0.000 & \#DIV/O! & 0.000 & 0.0 & 0.000 & \#DIV/o! & 0.000 & 0.0 \\
\hline Calcium & 1.399 & 0.000 & 00 & \#DIV/o! & 0.000 & 0.0 & 0.000 & \#DIVIo! & 0.000 & 0.0 & 0.000 & \#DIVIO! & 0.000 & 0.0 \\
\hline Chromium & 1.923 & 16.234 & 27.8 & 17.62 & 75.900 & 129.9 & 145.956 & 82.38 & 0.000 & 0.0 & 0.000 & 0.00 & 92.134 & 157.7 \\
\hline Cobalt & 1.271 & 0.000 & 0.0 & \#DIVIo! & 0.000 & 0.0 & 0.000 & \#DIV/O! & 0.000 & 0.0 & 0000 & \#DFio! & 0.000 & 0.0 \\
\hline Copper & 1.252 & 0.000 & 0.0 & \#DIV/o! & 0.000 & 0.0 & 0.000 & \#DIV/OI & 0.000 & 0.0 & 0.000 & \#DIV/o! & 0.000 & 0.0 \\
\hline Iron & 1.382 & 21.652 & 37.1 & 0.31 & 6864.000 & 11748.3 & 9486.048 & 99.69 & 0.000 & 0.0 & 0.000 & 0.00 & 6885.652 & 11785.3 \\
\hline Lead & 1.077 & 0.000 & 0.0 & \#DIV/O! & 0.000 & 0.0 & 0.000 & \#DIV/O! & 0.000 & 0.0 & 0.000 & \#DIVIO! & 0.000 & 00 \\
\hline Magnesium & 1.658 & 0.000 & 0.0 & \#DIV/0! & 0.000 & 0.0 & 0.000 & $\# D I V / O !$ & 0.000 & 0.0 & 0.000 & \#DIV/O! & 0.000 & 0.0 \\
\hline Manganese & 1.582 & 0.000 & 0.0 & \#DIVIor & 0.000 & 0.0 & 0.000 & HOIVID! & 0.000 & 0.0 & 0.000 & \#DIV/O| & 0.000 & 0.0 \\
\hline Nickel & 1.272 & 0.000 & 0.0 & \#DIV/o! & 0.000 & 0.0 & 0.000 & \#DIVIO! & 0.000 & 0.0 & 0.000 & \#DIVIO! & 0.000 & 0.0 \\
\hline Phosphate & 1.000 & 103594.650 & 177310.2 & 100.00 & 0.000 & 0.0 & 0.000 & 0.00 & 0.000 & 0.0 & 0.000 & 0.00 & 103594.650 & 177310.2 \\
\hline Potassium & 1.000 & 0.000 & 0.0 & \#DIV/0! & 0.000 & 0.0 & 0.000 & \#DIVIOI & 0.000 & 0.0 & 0.000 & \#DIVIO! & 0.000 & 0.0 \\
\hline Silicon & 2.139 & 56.820 & 97.3 & 2.59 & 2138.400 & 3660.0 & 4574.038 & 97.41 & 0.000 & 0.0 & 0.000 & 0.00 & 2195.220 & 3757.3 \\
\hline $\mathrm{H} 2 \mathrm{O}$ (hydration) & 1.000 & & 0.0 & \#DIV/OI & 0.000 & 0.0 & 0.000 & \#DIV/O! & 0.000 & 0.0 & 0.000 & \#DIV/0! & 0.000 & 0.0 \\
\hline Sodium & 1.000 & 190000.000 & 325199.6 & 98.65 & 2600.400 & 4450.8 & 2600.400 & 1.35 & 0.000 & 0.0 & 0.000 & 0.00 & 192600.400 & 329650.4 \\
\hline Strontium & 1.183 & 0.000 & 0.0 & \#DIV/o! & 0.000 & 0.0 & 0.000 & \#DIV/O! & 0.000 & 0.0 & 0000 & \#DIV/O! & 0.000 & 0.0 \\
\hline Zinc & 1.245 & 7.730 & 13.2 & 100,00 & 0.000 & 0.0 & 0.000 & 0.00 & 0.000 & 0.0 & 0.000 & 0.00 & 7.730 & 13.2 \\
\hline Zirconium & 1.351 & 0.000 & 0.0 & \#DIV/O! & 0.000 & 0.0 & 0.000 & \#DIV/O! & 0.000 & 0.0 & 0.000 & \#DIVIo! & 0.000 & 0.0 \\
\hline Uranium & 1.134 & 0.000 & 0.0 & 0.00 & 42.900 & 73.4 & 48.649 & 100.00 & 0.000 & 0.0 & 0.000 & 0.00 & 42.900 & 73.4 \\
\hline Nitrate & 1.000 & 125540.080 & 214871.5 & 100.00 & 0.000 & 0.0 & 0.000 & 0.00 & 0.000 & 0.0 & 0.000 & 0.00 & 125540.080 & 214871.5 \\
\hline Chloride & 1.000 & 0.000 & 0.0 & \#DIV/0! & 0.000 & 0.0 & 0.000 & \#DIVFor & 0.000 & 0.0 & 0.000 & \#DIVio! & 0.000 & 0.0 \\
\hline Fluoride & 1.000 & 0.000 & 0.0 & \#DrV/o! & 0.000 & 0.0 & 0.000 & \#OIVIO! & 0.000 & 0.0 & 0.000 & \#DIV/o! & 0.000 & 0.0 \\
\hline Free OH & 1.000 & 0.311 & 0.5 & 100.00 & 0.000 & 0.0 & 0.000 & 0.00 & 0.000 & 0.0 & 0.000 & 0.00 & $0.3 \uparrow 1$ & 0.5 \\
\hline Carbonate & 1.000 & 1338.000 & 2290.1 & 100.00 & 0.000 & 0.0 & 0.000 & 0.00 & 0.000 & 0.0 & 0.000 & 0.00 & 1338.000 & 2290.1 \\
\hline TOC & 1.336 & 0.000 & 0.0 & \#DIV/o! & 0.000 & 0.0 & 0.000 & $\#$ DIVI0! & 0.000 & 0.0 & 0.000 & $\#$ \#IV/O! & 0.000 & 0.0 \\
\hline Nitrite & 1.000 & 122070.200 & 208932.5 & 100.00 & 0.000 & 0.0 & 0.000 & 0.00 & 0.000 & 0.0 & 0.000 & 0.00 & 122070.200 & 208932.5 \\
\hline Sulfate & 1.000 & 15400.000 & 26358.3 & 100.00 & 0.000 & 0.0 & 0.000 & 0.00 & 0.000 & 0.0 & 0.000 & 0.00 & 15400.000 & 26358.3 \\
\hline Total & & $\begin{array}{r}558298.559 \\
96.26\end{array}$ & $\begin{array}{l}\% \text { total solids } \\
\text { in wash }\end{array}$ & & & & $\begin{array}{r}18413.515 \\
3.17\end{array}$ & $\begin{array}{l}\text { \% residual } \\
\text { solids }\end{array}$ & & & $\begin{array}{r}0.000 \\
0.00\end{array}$ & & 99.43 & $\begin{array}{l}\% \text { mass } \\
\text { balance }\end{array}$ \\
\hline Radionuclides & & $\mathrm{uCi} / \mathrm{g}$ & Ci/tank & dissolved & UCi/g & Ci/tank & & $\%$ dissolved & $\mathrm{uCl} / \mathrm{g}$ & Ci/tank & & $\%$ dissolved & $\mathrm{uCV} / \mathrm{g}$ & $\overline{\mathrm{C}} \mathrm{i} / \mathrm{tank}$ \\
\hline Pu-239,240 & & 0.000 & 0.0 & 000 & $6 . \overline{300}$ & 10782.9 & & 100.00 & 0.000 & 0.0 & & 0.00 & 6.300 & 10782.9 \\
\hline C-14 & & 0.000 & 0.0 & \#DIViol & NA & 0.0 & & HDIV/o! & 0.000 & 0.0 & & \#DIV/0! & 0.000 & 0.0 \\
\hline Sr.90 & & 1.035 & 1771.0 & 0.42 & 244.000 & $4\{7624.8$ & & 98.58 & 0.000 & 0.0 & & 0.00 & 245.035 & 419395.8 \\
\hline Tc-99 & & 0.000 & 0.0 & 0.00 & 0.009 & 14.7 & & 100.00 & 0.000 & 0.0 & & 0.00 & 0.009 & 14.7 \\
\hline Am-241 & & 0.000 & 0.0 & \#DIVIO! & NA & 0.0 & & \#DIVfo! & 0.000 & 0.0 & & \#OIVIo! & 0.000 & 0.0 \\
\hline Co-60 & & 0.000 & 0.0 & \#DIV/o! & NA & 0.0 & & \#DIV/O! & 0.000 & 0.0 & & HDIV/o! & 0.000 & 0.0 \\
\hline $\mathrm{Cs}-137$ & & 0.598 & 10229 & 0.68 & 97.000 & 148907.2 & & 99.32 & 0.000 & 0.0 & & 0.00 & 87.598 & 149930.1 \\
\hline $1-129$ & & 0.000 & 0.0 & \#DIV/O! & NA & 0.0 & & \#DIV/OI & 0.000 & 0.0 & & \#DIV/O! & 0.000 & 0.0 \\
\hline
\end{tabular}

NOTES:

Sulfate concentration derived trom $6 / 76$ sample analysis.

NR - Not Reported

Sodium has been adjusted from 5.48M to $3.84 \mathrm{M}$ for ion balance. 


\begin{tabular}{|c|c|c|c|c|c|c|c|c|c|c|c|c|c|c|}
\hline $\begin{array}{l}\left.\text { Density ( } \mathrm{g}^{\prime} \mathrm{m} \mathrm{L}\right) \\
\text { Volume }(\mathrm{L}) \\
\text { Mass (kg) } \\
\text { Solids (ug/g) } \\
\text { - pH }\end{array}$ & \begin{tabular}{r|}
1.10 \\
102195 \\
112415 \\
241000 \\
12.3 \\
\end{tabular} & & & & & & & & & & & & & \\
\hline Component & $\begin{array}{l}\text { Oxide } \\
\text { Factor }\end{array}$ & $\begin{array}{r}\text { ug/g sludge } \\
\text { (element) }\end{array}$ & $\begin{array}{r}\text { WaTER } \\
\text { (element) }\end{array}$ & $\begin{array}{c}\% \text { element } \\
\text { dissolved }\end{array}$ & $\begin{array}{l}\text { ug/g studge } \\
\text { (element) }\end{array}$ & $\begin{array}{r}\mathrm{kg} / \text { tank } \\
\text { (element) }\end{array}$ & $\begin{array}{l}\text { ug/g sludge } \\
\text { (oxide) }\end{array}$ & $\begin{array}{c}\text { \% element } \\
\text { dissolved }\end{array}$ & $\begin{array}{l}\text { ug/g sludge } \\
\text { (element) }\end{array}$ & $\begin{array}{c}\text { kg/tank } \\
\text { (element) }\end{array}$ & $\begin{array}{l}\text { g/g sludge } \\
\text { (oxide) }\end{array}$ & $\begin{array}{r}\text { \% element } \\
\text { dissolved }\end{array}$ & $\begin{array}{l}\text { ug/g sludge } \\
\text { (element) }\end{array}$ & $\begin{array}{r}\text { kg/tank } \\
\text { (element) }\end{array}$ \\
\hline Aluminum & $1.88 \dot{9}$ & 16.600 & 19 & 1.89 & 457.400 & 514 & 864.029 & 52.21 & 402.000 & 45.2 & 759.378 & 45.89 & 876.000 & 98.5 \\
\hline Barium & $\uparrow 117$ & 1.610 & 0.2 & 0.09 & 1778.390 & 199.9 & 1986.462 & 99.91 & 0.000 & 0.0 & 0.000 & 0.00 & 1780.000 & 200.1 \\
\hline Bismuth & 1.115 & 14.900 & 1.7 & 0.04 & 38885.100 & 4371.2 & 43356.887 & 99.96 & 0.000 & 0.0 & 0.000 & 0.00 & 38900.000 & 4372.9 \\
\hline Boron & 3.220 & 4720 & 05 & 3.47 & 131.280 & 14.8 & 422.722 & 96.53 & 0.000 & 0.0 & 0.000 & 0.00 & 136.000 & 15.3 \\
\hline Cadmium & 1.142 & 0.472 & 0.1 & 3.15 & 14.528 & 1.6 & 16.591 & 96.85 & 0.000 & 0.0 & 0.000 & 0.00 & 15.000 & 1.7 \\
\hline Calcium & 1.399 & 99.000 & 111 & 0.93 & 1541.000 & 173.2 & 2155.859 & 14.54 & 8960.000 & 1007.2 & 12535.040 & 84.53 & 10600.000 & 1191.6 \\
\hline Chromium & 1.923 & 707.000 & 79.5 & 21.30 & 2613.000 & 293.7 & 5024.799 & 78.70 & 0.000 & 00 & 0.000 & 0.00 & 3320.000 & 373.2 \\
\hline Cobalt & 1.271 & 0.840 & 0.1 & 6.94 & 2.260 & 0.3 & 2.872 & $\uparrow 8.68$ & 9.000 & 1.0 & 11.439 & 74.38 & 12.100 & 1.4 \\
\hline Copper & 1.252 & 0.522 & 0.1 & 1.28 & 13.778 & 15 & 17.250 & 33.69 & 26.600 & 3.0 & 33.303 & 65.04 & 40.900 & 4.6 \\
\hline Iron & 1.382 & 17.500 & 2.0 & 0.27 & 5372.500 & 603.9 & 7424.795 & 84.21 & 990.000 & 131.3 & 1368.180 & 15.52 & 6380.000 & 717.2 \\
\hline Lead & 1.077 & 9.650 & 1.1 & $\uparrow .57$ & 561.350 & 63.1 & 604.574 & 91.28 & 44.000 & 4.9 & 47.388 & 7.15 & 615.000 & 69.1 \\
\hline Magnesium & 1.658 & 5.210 & 0.6 & 0.97 & 252.790 & 28.4 & 419.126 & 46.90 & 281.000 & 31.6 & 465.898 & 52.13 & 539.000 & 60.6 \\
\hline Manganese & 1.582 & 2.680 & 0.3 & 0.02 & 13297.320 & 1494.8 & 21036.360 & 99.98 & 0.000 & 0.0 & 0.000 & 0.00 & 13300.000 & 1495.1 \\
\hline Nickel & 1.272 & 1.640 & 0.2 & 0.79 & 203.390 & 22.9 & 258.712 & 99.21 & 0.000 & 0.0 & 0.000 & 0.00 & 205.000 & 23.0 \\
\hline Phosphate & 1.000 & 2080.000 & 233.8 & 24.67 & 5930.000 & 666.6 & 5930.000 & 70.34 & 420.000 & 47.2 & 420.000 & 4.98 & 8430.000 & 947.7 \\
\hline Potassium & 1.000 & 6630.000 & 745.3 & 99.40 & 40.000 & 4.5 & 40.000 & 0.60 & 0.000 & 0.0 & 0.000 & 0.00 & 6670.000 & 749.8 \\
\hline Silicon & 2.139 & 795.000 & 89.4 & 26.59 & 0.000 & 0.0 & 0.000 & 0.00 & 2195.000 & 246.7 & 4695.105 & 73.41 & 2990.000 & 336.1 \\
\hline Lanthanum & 1.430 & 28.800 & 3.2 & 0.20 & 14271.200 & 1604.3 & 20407.816 & 99.80 & 0.000 & 0.0 & 0.000 & 0.00 & 14300.000 & 1607.5 \\
\hline Sodium & 1.000 & 36200.000 & 4069.4 & 96.79 & 1200.000 & 134.9 & 1200.000 & 3.21 & 0.000 & 0.0 & 0.000 & 0.00 & 37400.000 & 4204.3 \\
\hline Strontium & 1.193 & 2.280 & 0.3 & 0.38 & 592.720 & 66.6 & 701.188 & 99.62 & 0.000 & 0.0 & 0.000 & 0.00 & 595.000 & 66.9 \\
\hline Zinc & 1.245 & 9.230 & 1.0 & 2.06 & 33.170 & 3.7 & 41.297 & 7.39 & 406.600 & 45.7 & 506.217 & 90.56 & 449.000 & 50.5 \\
\hline Zirconium & 1.351 & 0.799 & 0.1 & 10.43 & 0.088 & 0.0 & 0.119 & 1.15 & 6.773 & 0.8 & 9.150 & 88.42 & 7.660 & 0.9 \\
\hline Uranium & 1.134 & 0.000 & 0.0 & 0.00 & 0.000 & 0.0 & 0.000 & 0.00 & 323.000 & 0.0 & 366.282 & 100.00 & 323.000 & 36.3 \\
\hline Nitrate & 1.000 & 61500.000 & 6913.5 & 100.00 & 0.000 & 0.0 & 0.000 & 0.00 & 0.000 & 0.0 & 0.000 & 0.00 & 61500.000 & 6913.5 \\
\hline Chloride & 1.000 & 864.000 & 97.1 & 100.00 & 0.000 & 0.0 & 0.000 & 0.00 & 0.000 & 0.0 & 0.000 & 0.00 & 864.000 & 97.1 \\
\hline Fluoride & 1.000 & 6240.000 & 701.5 & 100.00 & 0.000 & 0.0 & 0.000 & 0.00 & 0.000 & 0.0 & 0.000 & 0.00 & 6240.000 & 701.5 \\
\hline Free $\mathrm{OH}$ & 1.000 & 0.000 & 0.0 & \#D+V/O! & 0.000 & 0.0 & 0.000 & \#DIVIO! & 0.000 & 0.0 & 0000 & \#DIV/O? & 0.000 & 00 \\
\hline Carbonate & 1.000 & 1960.000 & 220.3 & 100.00 & 0.000 & 0.0 & 0.000 & 0.00 & 0.000 & 0.0 & 0.000 & 0.00 & 1960.000 & 220.3 \\
\hline TOC & 1.336 & 9770.000 & 737.9 & 100.00 & 0.000 & 0.0 & 0.000 & 0.00 & 0.000 & 0.0 & 0.000 & 0.00 & 6564.371 & 737.9 \\
\hline Nitrite & 1.000 & 1090.000 & 122.5 & 100.00 & 0.000 & 0.0 & 0.000 & 0.00 & 0.000 & 0.0 & 0.000 & 0.00 & 1090.000 & 122.5 \\
\hline Sulfate & 1.000 & 1530.000 & 172.0 & 74.78 & 0.000 & 0.0 & 0.000 & 0.00 & 516.000 & 58.0 & 516.000 & 25.22 & 2046.000 & 230.0 \\
\hline \multicolumn{2}{|l|}{ Total } & $\begin{array}{r}128582.423 \\
53.35 \\
\end{array}$ & \multicolumn{2}{|l|}{$\begin{array}{l}\text { \%total solids } \\
\text { in wash }\end{array}$} & \multicolumn{4}{|c|}{$\begin{array}{r}111911.456 \\
46.44\end{array}$} & & & $\begin{array}{r}21733.381 \\
9.02\end{array}$ & $\begin{array}{l}\text { \%total solids } \\
\text { in fusion }\end{array}$ & \multicolumn{2}{|l|}{108.81} \\
\hline \multicolumn{2}{|l|}{ Radionuclides } & uCi/g & Ci/tank & dissolved & $\mathrm{uCi} / \mathrm{g}$ & Ci/tank & & dissolved & $\mathrm{uCi} / \mathrm{g}$ & Ciltank & & dissolved & uCi/g & Ci/tank \\
\hline \multicolumn{2}{|l|}{$P y-239,240$} & NA & 0.0 & 0.00 & & 0.0 & & 0.00 & 0.153 & 77.2 & & 100.00 & 0.153 & 17.2 \\
\hline \multicolumn{2}{|l|}{$c-14$} & 0.0004 & 0.0 & 100.00 & & 0.0 & & 0.00 & 0.000 & 0.0 & & 0.00 & 0.000 & 0.0 \\
\hline \multicolumn{2}{|l|}{ Sr-90 } & NA & 0.0 & 0.00 & & 0.0 & & 0.00 & 4. 130 & 464.3 & & 100.00 & 4.130 & 464.3 \\
\hline \multicolumn{2}{|l|}{ Tc-99 } & NA & 0.0 & 0.00 & & 0.0 & & 0.00 & 0.005 & 0.6 & & 100.00 & 0.005 & 0.6 \\
\hline \multicolumn{2}{|l|}{ Am-241 } & NA & 0.0 & 0.00 & & 0.0 & & 100.00 & 0.068 & 7.7 & & 100.00 & 0.068 & 7.7 \\
\hline \multicolumn{2}{|l|}{ Co-60 } & NA & 0.0 & 0.00 & & 0.0 & & 0.00 & 0.010 & 1.1 & & 100.00 & 0.010 & 1.1 \\
\hline \multicolumn{2}{|l|}{ Cs-137 } & NA & 0.0 & 0.00 & & 0.0 & & 0.00 & 0.100 & 11.2 & & 100.00 & 0.100 & 11.2 \\
\hline \multicolumn{2}{|l|}{$1-129$} & NA & 0.0 & 0.00 & & 0.0 & & 0.00 & 0.016 & 1.8 & & 100.00 & 0.016 & 1.8 \\
\hline
\end{tabular}

NOTES: 
BX-409 (TBP/CW)

\begin{tabular}{|c|c|c|c|c|c|c|c|c|c|c|c|c|c|c|}
\hline $\begin{array}{l}\text { Density }(\mathrm{g} / \mathrm{mL} \text { ) } \\
\text { Volume }(\mathrm{L}) \\
\text { Mass }(\mathrm{kg}) \\
\text { Solids }(\mathrm{ug} / \mathrm{g}) \\
\sim \mathrm{pH} \\
\end{array}$ & $\begin{array}{r}1.50 \\
730505 \\
1095758 \\
497000 \\
\text { NR }\end{array}$ & & & & & & & & & & & & & \\
\hline Component & $\begin{array}{l}\text { Oxide } \\
\text { Factor }\end{array}$ & $\begin{array}{r}\text { ug/g sludge } \\
\text { (element) }\end{array}$ & $\begin{array}{r}\mathbf{k g} / \operatorname{tank} \\
\text { (etement) }\end{array}$ & $\begin{array}{c}\% \text { element } \\
\text { dissolved }\end{array}$ & $\begin{array}{r}\text { ug/g sludge } \\
\text { (element) }\end{array}$ & $\begin{array}{c}\text { kg/tank } \\
\text { (element) }\end{array}$ & $\begin{array}{r}\text { Ig/g sludge } \\
\text { (oxide) }\end{array}$ & $\begin{array}{r}\% \text { element } \\
\text { dissolved }\end{array}$ & $\begin{array}{r}\text { ug/g sludge } \\
\text { (element) }\end{array}$ & $\begin{array}{r}\text { OVERALL } \\
\text { kgitank } \\
\text { (eiement) }\end{array}$ & $\begin{array}{r}\text { MEAN -- } \\
\text { ug/g sludge } \\
\text { (oxide) }\end{array}$ & $\begin{array}{r}\% \text { etement } \\
\text { dissolved }\end{array}$ & $\begin{array}{r}\text { ug/g sludge } \\
\text { (element) }\end{array}$ & $\begin{array}{r}\text { kg/tank } \\
\text { (etement) }\end{array}$ \\
\hline Aluminum & 1.889 & 0.000 & 0.0 & 0.00 & 0.000 & 0.0 & 0.000 & 0.00 & 1910.000 & 2092.9 & 3607.990 & 100.00 & 1910.000 & 2092.9 \\
\hline Barium & 1117 & 0.000 & 0.0 & \#DIV/0! & 0.000 & 0.0 & 0.000 & \#DIV/0! & 0.000 & 0.0 & 0.000 & HDIV/0! & 0.000 & 0.0 \\
\hline Bismuth & 1.115 & 0.000 & 0.0 & \#DIV/o! & 0.000 & 0.0 & 0.000 & \#DIVIO! & 0.000 & 0.0 & 0.000 & \#DIV/O! & 0.000 & 0.0 \\
\hline Boron & 3220 & 0.000 & 0.0 & HDIV/O! & 0.000 & 0.0 & 0.000 & 韩IVIOI & 0.000 & 0.0 & 0.000 & \#DIV/O! & 0.000 & 0.0 \\
\hline Cadmium & 1.142 & 0.000 & 0.0 & 杖IV/O! & 0.000 & 0.0 & 0.000 & HDIV/O! & 0.000 & 0.0 & 0.000 & \#DIV/o! & 0.000 & 0.0 \\
\hline Calcium & 1.399 & 0.000 & 0.0 & 0.00 & 0.000 & 0.0 & 0.000 & 0.00 & 4035.000 & 4421.4 & 5644.965 & 100.00 & 4035.000 & 4421.4 \\
\hline Chromium & 1.923 & 0.000 & 0.0 & 0.00 & 0.000 & 0.0 & 0.000 & 0.00 & 137.000 & 1501 & 263.451 & 100.00 & 137.000 & 150.1 \\
\hline Cobalt & 1.271 & 0.000 & 0.0 & \#DIV/O! & 0.000 & 0.0 & 0.000 & HDIV/O! & 0.000 & 0.0 & 0.000 & HDIVIO! & 0.000 & 0.0 \\
\hline Copper & 1.252 & 0.000 & 0.0 & HDIV/O! & 0.000 & 0.0 & 0.000 & \#DIV/OI & 0.000 & 0.0 & 0.000 & \#DIV/o! & 0.000 & 0.0 \\
\hline Iron & 1.382 & 0.000 & 0.0 & 0.00 & 0.000 & 0.0 & 0.000 & 0.00 & 21500.000 & 23558.8 & 29713.000 & 100.00 & 21500.000 & 23558.8 \\
\hline Lead & 1.077 & 0.000 & 0.0 & 0.00 & 0.000 & 0.0 & 0.000 & 0.00 & 668.000 & 732.0 & 719.436 & 100.00 & 668.000 & 732.0 \\
\hline Magnesium & 1.658 & 0.000 & 0.0 & 0.00 & 0.000 & 0.0 & 0.000 & 0.00 & 748.000 & 819.6 & 1240.184 & 100.00 & 748.000 & 819.6 \\
\hline Manganese & 1.582 & 0.000 & 0.0 & \#D:V/o! & 0.000 & 0.0 & 0.000 & $\# \mathrm{D} \mid \mathrm{V} / 0 !$ & 0.000 & 0.0 & 0.000 & HDIV/o! & 0.000 & 0.0 \\
\hline Nickel & 1.272 & 0.000 & 0.0 & HDIV/O! & 0.000 & 0.0 & 0.000 & \#DIV/0! & 0.000 & 0.0 & 0.000 & \#D|V/o! & 0.000 & 0.0 \\
\hline Phosphate & 1.000 & 0.000 & 0.0 & 0.00 & 0.000 & 0.0 & 0.000 & 0.00 & 66000.000 & 723200 & 66000.000 & 100.00 & 66000.000 & 72320.0 \\
\hline Potassium & 1.000 & 0000 & 0.0 & \#DIVIO! & 0.000 & 0.0 & 0.000 & \#DIV/o! & 0.000 & 0.0 & 0.000 & \#DIV/O! & 0.000 & 0.0 \\
\hline Silicon & 2.139 & 0.000 & 0.0 & 0.00 & 0.000 & 0.0 & 0.000 & 0.00 & 1720.000 & 1884.7 & 3679.080 & 100.00 & 1720,000 & 1884.7 \\
\hline Silver & 1.000 & 0.000 & 0.0 & \#DIV/O! & 0.000 & 0.0 & 0.000 & \#DIV/O! & 0.000 & 0.0 & 0.000 & ADIVIO! & 0.000 & 0.0 \\
\hline Sodium & 1.000 & 0.000 & 0.0 & 0.00 & 0.000 & 0.0 & 0.000 & 0.00 & 114000.000 & 124916.4 & 114000.000 & 100.00 & 114000.000 & 1249164 \\
\hline Strontium & 1.183 & 0.000 & 0.0 & 0.00 & 0.000 & 0.0 & 0.000 & 0.00 & 590.000 & 646.5 & 697.970 & 100.00 & 590.000 & 646.5 \\
\hline Zine & 1.245 & 0.000 & 0.0 & 0.00 & 0.000 & 0.0 & 0.000 & 0.00 & 624.000 & 683.8 & 776.880 & 100.00 & 624.000 & 683.8 \\
\hline Zirconium & 1.351 & 0.000 & 0.0 & ADN/OI & 0.000 & 0.0 & 0.000 & \#DIVIOI & 0.000 & 0.0 & 0.000 & \#DIVIO! & 0.000 & 0.0 \\
\hline Uranium & 1.134 & 0.000 & 0.0 & 0.00 & 0.000 & 0.0 & 0.000 & 0.00 & 16200.000 & 0.0 & 18370.800 & 100.00 & 16200.000 & 17751.3 \\
\hline Nitrate & 1.000 & 0.000 & 0.0 & 0.00 & 0.000 & 0.0 & 0.000 & 0.00 & 213000.000 & 233396.3 & 213000.000 & 100.00 & 213000.000 & 233396.3 \\
\hline Chloride & 1.000 & 0.000 & 0.0 & 0.00 & 0.000 & 0.0 & 0.000 & 0.00 & 1200.000 & 1314.9 & 1200.000 & 100.00 & 1200.000 & 1314.9 \\
\hline Fluoride & 1.000 & 0.000 & 0.0 & HDIVIO! & 0.000 & 0.0 & 0.000 & HDIVIO1 & $<500$ & 0.0 & 0.000 & HDIVIO! & 0.000 & 0.0 \\
\hline Free OH & 1.000 & 0.000 & 0.0 & \#DIVo! & 0.000 & 0.0 & 0.000 & HDIV/O! & 0.000 & 0.0 & 0.000 & \#DIV/OI & 0.000 & 0.0 \\
\hline Carbonate & 1.000 & 0.000 & 0.0 & ADIV/O! & 0.000 & 0.0 & 0.000 & \#DIV/0! & 0.000 & 0.0 & 0.000 & HDIV/O! & 0.000 & 0.0 \\
\hline TOC & 1.336 & 0.000 & 0.0 & HDIV/O! & 0.000 & 0.0 & 0.000 & tolvio! & 0.000 & 0.0 & 0.000 & $\# \mathrm{DIV} / 0$ ! & 0.000 & 0.0 \\
\hline Nitrite & 1.000 & 0.000 & 0.0 & 0.00 & 0.000 & 0.0 & 0.000 & 0.00 & 19100.000 & 20929.0 & 19100.000 & 100.00 & 19100.000 & 20929.0 \\
\hline Sulfate & 1.000 & 0.000 & 0.0 & 0.00 & 0.000 & 0.0 & 0.000 & 0.00 & 18800.000 & 20600.2 & 48800.000 & 100.00 & 18800000 & 20600.2 \\
\hline Total & & $\begin{array}{r}0.000 \\
0.00\end{array}$ & $\begin{array}{l}\text { \%ototal solids } \\
\text { in wash }\end{array}$ & & & & $\begin{array}{r}0.000 \\
0.00\end{array}$ & $\begin{array}{l}\text { \%ototal solids } \\
\text { in acid }\end{array}$ & & & $\begin{array}{r}496813.756 \\
99.96\end{array}$ & $\begin{array}{l}\text { \%total solids } \\
\text { in fussion }\end{array}$ & 99.96 & $\begin{array}{l}\% \text { mass } \\
\text { balance }\end{array}$ \\
\hline Radionuclides & & uCilg & Ci/tank & dissolved & $\mathrm{uCl} / \mathrm{g}$ & Ci/tank & & dissolved & uCi/g & Cî́tank & & dissolved & uCiig & Ci/tank \\
\hline Total Alpha & & 0.000 & 0.0 & \#DIV/o! & & 0.0 & & \#DIVIo! & $<0.05$ & 0.0 & & \#DIV/어 & 0.000 & 0.0 \\
\hline C-14 & & 0.000 & 0.0 & HDIV/O1 & & 0.0 & & HDIVIO! & 0.000 & 0.0 & & $\#$ DIV/01 & 0.000 & 0.0 \\
\hline Sr-90 & & 0.000 & 0.0 & 0.00 & & 0.0 & & 0.00 & 178.000 & 195044.8 & & 100.00 & 178.000 & 195044.8 \\
\hline TC-99 & & 0.000 & 0.0 & \#D|V/o! & & 0.0 & & $\# \mathrm{D} \mid V / 0 !$ & 0.000 & 0.0 & & \#DIVIO! & 0.000 & 0.0 \\
\hline Am-241 & & 0.000 & 0.0 & 杖1V/o! & & 0.0 & & HDIVIO! & $<1.26$ & 0.0 & & HDIV/0! & 0.000 & 0.0 \\
\hline Co-60 & & 0.000 & 0.0 & HDIVIOI & & 0.0 & & \#DIV/0! & $<0.13$ & 0.0 & & ADIV/O! & 0.000 & 0.0 \\
\hline Cs-137 & & 0.000 & 0.0 & 0.00 & & 0.0 & & 0.00 & 13.900 & 15231.0 & & 100.00 & 13.900 & 15231.0 \\
\hline $1-129$ & & 0.000 & 0.0 & \#DIV/OI & & 0.0 & & \#DIVIO! & 0.000 & 0.0 & & \#DIVIOl & 0.000 & 0.0 \\
\hline
\end{tabular}

NOTES: Field, J. G., R. D. Schreiber, B. C. Simpson. 1996. Tank Characterization Report for Single-shell Tank 241-BX-109, NR - Not Reparted
WHC-SD-WM-ER-572, Rev. 0. Westinghouse Hanford Company, Richland, Washington. 


\begin{tabular}{|c|c|c|c|c|c|c|c|c|c|c|c|c|c|c|}
\hline \begin{tabular}{|l} 
Density $(\mathrm{g} / \mathrm{mL})$ \\
Volume $(\mathrm{L})$ \\
Mass $(\mathrm{kg})$ \\
Solids (ug/g) \\
$-\mathrm{pH}$ \\
\end{tabular} & $\begin{array}{r}1.60 \\
1536710 \\
2458736 \\
770000 \\
\mathrm{NR} \\
\end{array}$ & & & & & & & & & & & & & \\
\hline & & & WATER & 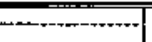 & & $A C$ & . & & & Füs| & ON -.... & "ב-.--. & - & AL -...-..- \\
\hline Comporrent & $\begin{array}{l}\text { Oxide } \\
\text { Factor }\end{array}$ & $\begin{array}{r}\text { ug/g sludge } \\
\text { (element) }\end{array}$ & $\begin{array}{r}\mathrm{kg} / \tan \mathrm{k} \\
\text { (element) }\end{array}$ & $\begin{array}{r}\% \text { element } \\
\text { dissolved }\end{array}$ & $\begin{array}{r}\text { ug/g sludge } \\
\text { (element) }\end{array}$ & $\begin{array}{c}\text { kg/tank } \\
\text { (element) }\end{array}$ & $\begin{array}{r}\text { g sludge } \\
\text { (oxide) }\end{array}$ & $\begin{array}{r}\% \text { element } \\
\text { dissolved }\end{array}$ & $\begin{array}{r}\text { ug/g sludge } \\
\text { (element) }\end{array}$ & $\begin{array}{r}\mathrm{kg} / \operatorname{tank} \\
\text { (element) }\end{array}$ & $\begin{array}{r}\text { ug/g sludge } \\
\text { (oxide) }\end{array}$ & $\begin{array}{r}\% \text { element } \\
\text { dissolved }\end{array}$ & $\begin{array}{r}\text { ug/g sludge } \\
\text { (element) }\end{array}$ & $\begin{array}{r}\mathrm{kg} / \text { tank } \\
\text { (element) }\end{array}$ \\
\hline Aluminum & 1.889 & 0.000 & 0.0 & 0.00 & 0.000 & 0.0 & 0.000 & 0.00 & 47950.000 & 117896.4 & 90577.550 & 100.00 & 47950.000 & 1177896.4 \\
\hline Barium & 1.117 & 0.000 & 0.0 & 0.00 & 0.000 & 0.0 & 0.000 & 0.00 & 31.000 & 76.2 & 34.627 & 100.00 & 31.000 & 76.2 \\
\hline Bismuth & 1.115 & 0.000 & 0.0 & \#DN/O! & 0.000 & 00 & 0.000 & HDIV/O! & NR & 0.0 & 0.000 & \#DIV/0! & 0.000 & 0.0 \\
\hline Boron & 3.220 & 0.000 & 0.0 & \#DIV/o! & 0.000 & 0.0 & 0.000 & \#DIV/0! & 0.000 & 0.0 & 0.000 & \#DIV/O! & 0.000 & 0.0 \\
\hline Cadmium & 1.142 & 0.000 & 0.0 & 0.00 & 0.000 & 0.0 & 0.000 & 0.00 & 69.500 & 170.9 & 79.369 & 100.00 & 69.500 & 170.9 \\
\hline Calcium & 1.399 & 0.000 & 0.0 & 0.00 & 0.000 & 0.0 & 0.000 & 0.00 & 1112.500 & 2735.3 & 1556.388 & 100.00 & 1112.500 & 2735.3 \\
\hline Chromium & 1.923 & 0.000 & 0.0 & 0.00 & 0.000 & 0.0 & 0.000 & 0.00 & 5800.000 & 142607 & 11153.400 & 100.00 & 5800.000 & 14260.7 \\
\hline Cobalt & $1.27 t$ & 0.000 & 0.0 & 0.00 & 0.000 & 0.0 & 0.000 & 0.00 & 36.500 & 89.7 & 46.392 & 100.00 & 36.500 & 89.7 \\
\hline Copper & 1.252 & 0.000 & 0.0 & \#DIV/0! & 0.000 & 0.0 & 0.000 & \#DIV/0! & 0.000 & 0.0 & 0.000 & HDIVI0! & 0.000 & 0.0 \\
\hline Iron & 1.382 & 0.000 & 0.0 & 0.00 & 0.000 & 0.0 & 0.000 & 0.00 & 4790.000 & 11777.3 & 6619.780 & 100.00 & 4790.000 & 11777.3 \\
\hline Lead & 1.077 & 0.000 & 0.0 & \#DIVfol & 0.000 & 0.0 & 0.000 & HDIV/o! & 0.000 & 0.0 & 0.000 & ADIV/O? & 0.000 & 0.0 \\
\hline Magnesium & 1.658 & 0.000 & 0.0 & \#DIV/O! & 0.000 & 0.0 & 0.000 & \#DIVI0! & 0.000 & 0.0 & 0.000 & \#DIV/O! & 0.000 & 0.0 \\
\hline Manganese & 1.582 & 0.000 & 0.0 & \#DIV/0! & 0.000 & 0.0 & 0.000 & HOIVIO! & 0.000 & 0.0 & 0.000 & \#DIVfo! & 0.000 & 0.0 \\
\hline Nickel & 1.272 & 0.000 & 0.0 & 0.00 & 0.000 & 0.0 & 0.000 & 0.00 & 920.000 & 2262.0 & 1170.240 & 100.00 & 920.000 & 2262.0 \\
\hline Phosphate & 1.000 & 0.000 & 0.0 & 0.00 & 0.000 & 0.0 & 0.000 & 0.00 & 15735.000 & 38688.2 & 15735.000 & 100.00 & 15735.000 & 38688.2 \\
\hline Potassium & 1.000 & 0.000 & 0.0 & 0.00 & 0.000 & 0.0 & 0.000 & 0.00 & 2650.000 & 6515.7 & 2650.000 & 100.00 & 2650.000 & 6515.7 \\
\hline Silicon & 2.139 & 0.000 & 0.0 & 0.00 & 0.000 & 0.0 & 0.000 & 0.00 & 1494.500 & 3674.6 & 3196.736 & 100.00 & 1494.500 & 3674.6 \\
\hline Silver & 1.000 & 0.000 & 0.0 & \#DIV/0! & 0.000 & 0.0 & 0.000 & HDIVIO! & 0.000 & 0.0 & 0.000 & \#DIV $/ 0 !$ & 0.000 & 0.0 \\
\hline Sodium & $\uparrow .000$ & 0.000 & 0.0 & 0.00 & 0.000 & 0.0 & 0.000 & 0.00 & 225000.000 & 553215.6 & 225000.000 & 100.00 & 225000.000 & 553215.6 \\
\hline Strontium & 1.183 & 0.000 & 0.0 & 0.00 & 0.000 & 0.0 & 0.000 & 0.00 & 1157.000 & 2844.8 & 1368.731 & 100.00 & 1157.000 & 2844.8 \\
\hline Zinc & 1.245 & 0.000 & 0.0 & 0.00 & 0.000 & 0.0 & 0.000 & 0.00 & 250.000 & 614.7 & 311.250 & 100.00 & 250.000 & 614.7 \\
\hline Zirconium & 1.351 & 0.000 & 0.0 & 0.00 & 0.000 & 0.0 & 0.000 & 0.00 & 53.000 & 130.3 & 71.603 & 10000 & 53.000 & 130.3 \\
\hline Uranium & 1.134 & 0.000 & 0.0 & 0.00 & 0.000 & 0.0 & 0.000 & 0.00 & 2682.500 & 0.0 & 3041.955 & 100.00 & 2682.500 & 6595.6 \\
\hline Nitrate & 1.000 & 0.000 & 0.0 & 0.00 & 0.000 & 0.0 & 0.000 & 0.00 & 61775.000 & 151888.4 & 61775.000 & 100.00 & 61775.000 & 151888.4 \\
\hline Chloride & 1.000 & 0.000 & 0.0 & 0.00 & 0.000 & 0.0 & 0.000 & 0.00 & 1557.500 & 3829.5 & 1557.500 & 100.00 & 1557.500 & 3829.5 \\
\hline Fluoride & 1.000 & 0.000 & 0.0 & 0.00 & 0.000 & 0.0 & 0.000 & 0.00 & 9417.500 & 23155.1 & 9417.500 & 100.00 & 9417.500 & 23155.1 \\
\hline $\mathrm{Free} \mathrm{OH}$ & 1.000 & 0.000 & 0.0 & \#DIV/0! & 0.000 & 0.0 & 0.000 & \#DIVI0! & 0.000 & 0.0 & 0.000 & \#DIV/0! & 0.000 & 0.0 \\
\hline Carbonate & 1.000 & 0.000 & 0.0 & 0.00 & 0.000 & 0.0 & 0.000 & 0.00 & 191000.000 & 469618.6 & 191000.000 & 100.00 & 191000.000 & 469618.6 \\
\hline TOC & 1.336 & 0.000 & 0.0 & 0.00 & 0.000 & 0.0 & 0.000 & 0,00 & 10050.000 & 24710.3 & 43426.800 & 100.00 & 10050.000 & 24710.3 \\
\hline Nitrite & 1.000 & 0.000 & 0.0 & 0.00 & 0.000 & 0.0 & 0.000 & 0.00 & 13800.000 & 33930.6 & 13800.000 & 100.00 & 13800.000 & 33930.6 \\
\hline Sutfate & 1.000 & 0.000 & 0.0 & 0.00 & 0.000 & 0.0 & 0.000 & 0.00 & 35550.000 & 87408.1 & 35550.000 & 100.00 & 35550.000 & 87408.1 \\
\hline \multicolumn{2}{|l|}{ Totat } & $\begin{array}{r}0.000 \\
0.00 \\
\end{array}$ & \multicolumn{2}{|l|}{$\begin{array}{l}\text { \%total solids } \\
\text { in wash }\end{array}$} & & & $\begin{array}{r}0.000 \\
0.00\end{array}$ & $\begin{array}{l}\text { \%total solids } \\
\text { in acid }\end{array}$ & & & $\begin{array}{r}689139.820 \\
89.50\end{array}$ & $\begin{array}{l}\text { \%total solids } \\
\text { in fusion }\end{array}$ & 89.50 & $\begin{array}{l}\% \text { mass } \\
\text { balance }\end{array}$ \\
\hline \multicolumn{2}{|l|}{ Rađionuclides } & uCV/g & Ci/tank & dissolved & $\mathrm{uCi} / \mathrm{g}$ & $\mathrm{Cl} /$ tank & & dissolved & $\mathrm{uCi} / \mathrm{g}$ & Ciltank & & dissolved & $\mathrm{uCi} / \mathrm{g}$ & Ci/tank \\
\hline \multicolumn{2}{|l|}{ Pu-239,240 } & 0.000 & 0.0 & \#DIVio! & & 0.0 & & \#DIV/O! & 0.000 & 0.0 & & \#DIV/0! & 0.000 & 0.0 \\
\hline \multicolumn{2}{|l|}{ C-14 } & 0.000 & 0.0 & $\#$ DIVIO! & & 0.0 & & \#DIV/0! & 0.000 & 0.0 & & \#DIV/0! & 0.000 & 0.0 \\
\hline \multicolumn{2}{|l|}{ Sr.90 } & 0.000 & 0.0 & HDIVIO! & & 0.0 & & \#DIV/O! & 0.000 & 0.0 & & HDIV/O1 & 0.000 & 0.0 \\
\hline \multicolumn{2}{|l|}{ TC-99 } & 0.000 & 0.0 & HDIV/0! & & 0.0 & & \#DIV/0! & 0.000 & 0.0 & & \#DIVIO! & 0.000 & 0.0 \\
\hline \multicolumn{2}{|l|}{ Amr-24† } & 0.000 & 0.0 & HDIVIO! & & 0.0 & & \#DIV/OI & 0.000 & 0.0 & & HDIVIO! & 0.000 & 0.0 \\
\hline \multicolumn{2}{|l|}{$\mathrm{Co}-60$} & 0.000 & 0.0 & \#DIV/0! & & 0.0 & & \#DIVIO! & 0.000 & 0.0 & & HDIV/OI & 0.000 & 0.0 \\
\hline \multicolumn{2}{|l|}{ Cs-137 } & 0.000 & 0.0 & \#DIV/OI & & 0.0 & & HDIV/01 & 0.000 & 0.0 & & HDIVI0! & 0.000 & 0.0 \\
\hline \multicolumn{2}{|l|}{$1-129$} & 0.000 & 0.0 & \#DIV/0! & & 0.0 & & \#DIVIOI & 0.000 & 0.0 & & \#DIV/OI & 0.000 & 0.0 \\
\hline
\end{tabular}


BY-110 [TBP(f), EB]

\begin{tabular}{|c|c|c|c|c|c|c|c|c|c|c|c|c|c|c|}
\hline \begin{tabular}{|l} 
Density $(\mathrm{g} / \mathrm{mL})$ \\
Volume $(\mathrm{L})$ \\
Mass $(\mathrm{kg})$ \\
Solids $(\mathrm{ug} / \mathrm{g})$ \\
$\sim \mathrm{pH}$ \\
\end{tabular} & $\begin{array}{r}1.50 \\
1506430 \\
2259645 \\
819000\end{array}$ & & & & & & & & & & & & & \\
\hline Component & $\begin{array}{l}\text { Oxide } \\
\text { Factor }\end{array}$ & $\begin{array}{r}\text { ug/g sludge } \\
\text { (element) }\end{array}$ & $\begin{array}{r}\mathrm{kg} / \mathrm{tank} \\
\text { (element) }\end{array}$ & $\begin{array}{r}\% \text { eiement } \\
\text { dissolved }\end{array}$ & $\begin{array}{r}\text { ugfg sludge } \\
\text { (element) }\end{array}$ & $\begin{array}{c}\text { kg/tank ug/ } \\
\text { (element) }\end{array}$ & $\begin{array}{r}9 \text { sludge } \\
\text { (oxide) }\end{array}$ & $\begin{array}{r}\% \text { element } \\
\text { dissolved }\end{array}$ & $\begin{array}{r}\text { ug/g sludge } \\
\text { (element) }\end{array}$ & $\begin{array}{r}\text { Average Se } \\
\mathrm{kg} / \text { tank } \\
\text { (element) }\end{array}$ & $\begin{array}{r}\text { gments - } \\
\text { ug/g sludge } \\
\text { (oxide) }\end{array}$ & $\begin{array}{c}\% \text { element } \\
\text { dissolved }\end{array}$ & $\begin{array}{r}\text { ug/g sludge } \\
\text { (element) }\end{array}$ & $\begin{array}{r}\mathrm{kg} / \mathrm{tank} \\
\text { (element) }\end{array}$ \\
\hline Aluminum & 2.720 & 0.000 & 0.0 & 0.00 & 0.000 & 0.0 & 0.000 & 0.00 & 13200000 & 29827.3 & 35904.000 & 100.00 & 13200.000 & 29827.3 \\
\hline Barium & 1.117 & 0.000 & 0.0 & 0.00 & 0.000 & 0.0 & 0.000 & 0.00 & 58.800 & 132.9 & 65.680 & 100.00 & 58.800 & 132.9 \\
\hline Bismuth & 1.115 & 0.000 & 0.0 & 0.00 & 0.000 & 0.0 & 0.000 & 0.00 & 931.000 & 2103.7 & 1038.065 & 100.00 & 931.000 & 2103.7 \\
\hline Boron & 3.220 & 0.000 & 0.0 & 0.00 & 0.000 & 0.0 & 0.000 & 0.00 & 79.900 & 180.5 & 257.278 & 100.00 & 79.900 & 180.5 \\
\hline Cadmiun & 1142 & 0.000 & 0.0 & 0.00 & 0.000 & 0.0 & 0.000 & 0.00 & 22.100 & 49.9 & 25.238 & 100.00 & 22.100 & 49.9 \\
\hline Calcium & 1.399 & 0.000 & 0.0 & 0.00 & 0.000 & 0.0 & 0.000 & 000 & 1110000 & 2508.2 & 1552.890 & 100.00 & 1110.000 & 2508.2 \\
\hline Chromium & 1923 & 0.000 & 0.0 & 0.00 & 0.000 & 0.0 & 0.000 & 0.00 & 2780.000 & 6281.8 & 5345.940 & 10000 & 2780.000 & 6281.8 \\
\hline Cobalt & 1.271 & 0.000 & 0.0 & 0.00 & 0.000 & 0.0 & 0.000 & 000 & 26.600 & 60.1 & 33.809 & 100.00 & 26.600 & 601 \\
\hline Copper & 1.252 & 0.000 & 00 & 0.00 & 0.000 & 0.0 & 0.000 & 0.00 & 12.200 & 27.6 & 15.274 & 100.00 & 12.200 & 27.6 \\
\hline Iron & 1.382 & 0.000 & 0.0 & 0.00 & 0000 & 0.0 & 0.000 & 0.00 & 961.000 & 2171.5 & 1328.102 & 100.00 & 961.000 & 21715 \\
\hline Lead & 1.077 & 0.000 & 0.0 & 0.00 & 0.000 & 0.0 & 0.000 & 0.00 & 962.000 & 2173.8 & 1036.074 & 100.00 & 962.000 & 2173.8 \\
\hline Magnesium & 1.658 & 0.000 & 0.0 & 0.00 & 0.000 & 0.0 & 0.000 & 0.00 & 120.000 & 271.2 & 198.960 & 100.00 & 120.000 & 271.2 \\
\hline Manganese & 1.582 & 0.000 & 0.0 & 0.00 & 0.000 & 0.0 & 0.000 & 0.00 & 48.800 & 110.3 & 77.202 & 100.00 & 48.800 & $\$ 10.3$ \\
\hline Nickel & 1.272 & 0.000 & 0.0 & 0.00 & 0.000 & 0.0 & 0.000 & 0.00 & 301.000 & 680.2 & 382.872 & 100.00 & 301.000 & 680.2 \\
\hline Phosphate & 1.000 & 0.000 & 0.0 & 0.00 & 0.000 & 0.0 & 0.000 & 0.00 & 9840.000 & 22234.9 & 9840.000 & 100.00 & 9840.000 & 222349 \\
\hline Potassium & 1.000 & 0.000 & 0.0 & 0.00 & 0.000 & 0.0 & 0000 & 0.00 & 1820.000 & 4112.6 & 1820.000 & 100.00 & 1820.000 & 4112.6 \\
\hline Silicon & 2.139 & 0.000 & 0.0 & 0.00 & 0.000 & 0.0 & 0.000 & 0.00 & 685.000 & 1547.9 & 1465.215 & 100.00 & 685.000 & 1547.9 \\
\hline Silver & 1.000 & 0.000 & 0.0 & 0.00 & 0.000 & 0.0 & 0.000 & 0.00 & 14.400 & 32.5 & 14.400 & 100.00 & 14.400 & 32.5 \\
\hline Sodium & 1.000 & 0.000 & 0.0 & 0.00 & 0.000 & 0.0 & 0.000 & 0.00 & 254000.000 & 573949.8 & 254000.000 & 100.00 & 254000.000 & 573949.8 \\
\hline Strontium & 1.183 & 0.000 & 0.0 & 0.00 & 0.000 & 0.0 & 0.000 & 0.00 & 58.500 & 132.2 & 69.206 & 100.00 & 58.500 & 132.2 \\
\hline Zinc & 1.245 & 0.000 & 0.0 & 0.00 & 0.000 & 0.0 & 0.000 & 0,00 & 39.900 & 90.2 & 49.676 & 100.00 & 39.900 & 90.2 \\
\hline Zirconium & 1.351 & 0.000 & 0.0 & 0.00 & 0.000 & 0.0 & 0.000 & 0.00 & 15.800 & 35.7 & 21.346 & 100.00 & 15.800 & 35.7 \\
\hline Uranitum & 1.134 & 0.000 & 0.0 & 0.00 & 0.000 & 0.0 & 0.000 & 0.00 & 4140.000 & 0.0 & 4694.760 & 100.00 & $4 \uparrow 40.000$ & 9354.9 \\
\hline Nitrate & 1.000 & 0.000 & 0.0 & 0.00 & 0.000 & 0.0 & 0.000 & 0.00 & 216000.000 & 488083.3 & 216000.000 & 100.00 & 216000.000 & 488083.3 \\
\hline Chloride & 1.000 & 0.000 & 0.0 & 0.00 & 0.000 & 0.0 & 0.000 & 0.00 & 1640.000 & 3705.8 & 1640.000 & 100.00 & 1640.000 & 3705.8 \\
\hline Fluoride & 1.000 & 0.000 & 0.0 & 0.00 & 0.000 & 0.0 & 0.000 & 0.00 & 6450.000 & 14574.7 & 6450.000 & 100.00 & 6450.000 & 14574.7 \\
\hline Free OH & 1.000 & 0.000 & 0.0 & \#DNV/0! & 0.000 & 0.0 & 0.000 & HDIV/0! & 0.000 & 0.0 & 0.000 & $\# D \mid V / 0 !$ & 0.000 & 0.0 \\
\hline Carbonate & 1.000 & 0.000 & 0.0 & 0.00 & 0.000 & 0.0 & 0.000 & 0.00 & 191000.000 & 431592.2 & 191000.000 & 100.00 & 191000.000 & 431592.2 \\
\hline Oxalate & 1.000 & 0.000 & 0.0 & 0.00 & 0.000 & 0.0 & 0.000 & 0.00 & 18200.000 & 41125.5 & 18200.000 & 100.00 & 18200.000 & 41125.5 \\
\hline Nitrite & 1.000 & 0.000 & 0.0 & 0.00 & 0.000 & 0.0 & 0.000 & 0.00 & 22500.000 & 50842.0 & 22500.000 & 100.00 & 22500.000 & 50842.0 \\
\hline Sulfate & 1.000 & 0.000 & 0.0 & 0.00 & 0.000 & 0.0 & 0.000 & 0.00 & 23200.000 & 52423.8 & 23200.000 & 100.00 & 23200.000 & 52423.8 \\
\hline Total & & $\begin{array}{r}0.000 \\
0.00\end{array}$ & $\begin{array}{l}\text { \%total solids } \\
\text { in wash }\end{array}$ & & & & $\begin{array}{r}0.000 \\
0.00 \\
\end{array}$ & $\begin{array}{l}\text { \%total solids } \\
\text { in acid }\end{array}$ & & & $\begin{array}{r}798225.985 \\
97.46 \\
\end{array}$ & $\begin{array}{l}\text { \%ototal solids } \\
\text { in fusion }\end{array}$ & 97.46 & $\begin{array}{l}\% \text { mass } \\
\text { balance }\end{array}$ \\
\hline Radionuclides & & $\mathrm{uCl} / \mathrm{g}$ & Ci/tank & dissolved & uCi/g & Ci/tank & & dissolved & $\mathrm{uCi} / \mathrm{g}$ & Ci/tank & & dissolved & uCi/g & Ci/tank \\
\hline $\mathrm{Pu}-239.240$ & & 0.000 & 0.0 & 0.00 & & 0.0 & & 0.00 & 0.071 & 159.5 & & 100.00 & 0.071 & 159.5 \\
\hline C-14 & & 0.000 & 0.0 & \#DIV/O! & & 0.0 & & \#DIVIO! & 0.000 & 0.0 & & $\#$ DIV/O! & 0.000 & 0.0 \\
\hline Sr-90 & & 0.000 & 0.0 & 0.00 & & 0.0 & & 0.00 & 21.000 & 47452.5 & & 100.00 & 21.000 & 47452.5 \\
\hline TC-99 & & 0.000 & 0.0 & HDIVIO! & & 0.0 & & \#DIVIO! & 0.000 & 0.0 & & $\#$ CIV/o! & 0.000 & 0.0 \\
\hline$A m-24 \uparrow$ & & 0.000 & 0.0 & 0.00 & & 0.0 & & 0.00 & 0.409 & 924.2 & & 100.00 & 0.409 & 924.2 \\
\hline Co-60 & & 0.000 & 0.0 & 0.00 & & 0.0 & & 0.00 & 0.019 & 43.6 & & 100.00 & 0.019 & 43.6 \\
\hline Cs-137 & & 0.000 & 0.0 & 0.00 & & 0.0 & & 0.00 & 57.900 & 130833.4 & & 100.00 & 57.900 & 130833.4 \\
\hline I-129 & & 0.000 & 0.0 & \#DIVIO! & & 0.0 & & \#DIVIO! & 0.000 & 0.0 & & \#DIV/O! & 0.000 & 0.0 \\
\hline
\end{tabular}

Noticeably targe concentration of $\mathrm{CO}\left([\mathrm{TIC}]{ }^{*} 5\right)$; consistent with ion balance.

NA - No Analysis Performed 
C-107 (BiPO4 1C, CW)

\begin{tabular}{|c|c|c|c|c|c|c|c|c|c|c|c|c|c|}
\hline \begin{tabular}{|l} 
Density $(\mathrm{g} / \mathrm{mL})$ \\
Volume $(\mathrm{L})$ \\
Mass $(\mathrm{kg})$ \\
Solids $(\mathrm{ug} / \mathrm{g})$ \\
$-\mathrm{pH}$
\end{tabular} & $\begin{array}{r}1.50 \\
897045 \\
1345568 \\
1000000\end{array}$ & dried sample & $=1 \% \mathrm{H} 2 \mathrm{O}-54 \%$ & & & & & & & & & & \\
\hline Component & $\begin{array}{l}\text { Oxide } \\
\text { Factor }\end{array}$ & $\begin{array}{r}\text { ug/g sludge } \\
\text { (element) }\end{array}$ & $\begin{array}{r}\mathrm{kg} / \operatorname{tank} \\
\text { (element) }\end{array}$ & $\begin{array}{c}\text { \% element } \\
\text { dissolved }\end{array}$ & $\begin{array}{r}\text { ug/g sludge } \\
\text { (element) }\end{array}$ & $\begin{array}{c}\text { kg/lank us } \\
\text { (element) }\end{array}$ & $\begin{array}{l}g \text { sludge } \\
\text { (oxide) }\end{array}$ & $\begin{array}{l}\% \text { element } \\
\text { dissolved }\end{array}$ & $\begin{array}{r}\begin{array}{r}\text { ug/g sludge } \\
\text { (element) }\end{array} \\
\end{array}$ & $\begin{array}{r}\text { LANL Direc } \\
\text { kg/tank } \\
\text { (element) }\end{array}$ & $\begin{array}{l}\text { ug } / g \text { sludge } \\
\text { (oxide) }\end{array}$ & $\begin{array}{r}\text { ug/g sludge } \\
\text { (element) }\end{array}$ & $\begin{array}{r}\mathrm{kg} / \operatorname{tank} \\
\text { (element) }\end{array}$ \\
\hline Aluminum & 2.720 & 0.000 & 0.0 & 0.00 & 0.000 & 0.0 & 0.000 & 0.00 & 105000.000 & 941284.6 & 285600.000 & 105000.000 & 141284.6 \\
\hline Barium & 1.117 & 0.000 & 0.0 & 0.00 & 0.000 & 0.0 & 0.000 & 0.00 & 835.000 & 1123.5 & 932.695 & 835.000 & 1123.5 \\
\hline Bismuth & 1.115 & 0.000 & .0 .0 & 0.00 & 0.000 & 0.0 & 0.000 & 0.00 & 24900.000 & 33504.6 & 27763.500 & 24900.000 & 33504.6 \\
\hline Boron & 3.220 & 0.000 & 0.0 & 0.00 & 0.000 & 0.0 & 0.000 & 0.00 & 5.850 & 7.9 & 18.837 & 5.850 & 7.9 \\
\hline Cadmium & 1.142 & 0.000 & 0.0 & 0.00 & 0.000 & 0.0 & 0.000 & 0.00 & 101.000 & 135.9 & 115.342 & 101.000 & 135.9 \\
\hline Calcium & 1.399 & 0.000 & 0.0 & 0.00 & 0.000 & 0.0 & 0.000 & 0.00 & 973.000 & 1309.2 & 1361.227 & 973.000 & 1309.2 \\
\hline Chromium & 1.923 & 0.000 & 0.0 & 0.00 & 0.000 & 0.0 & 0.000 & 0.00 & 706.000 & 950.0 & 1357.638 & 706.000 & 950.0 \\
\hline Cobalt & 1.271 & 0.000 & 0.0 & 0.00 & 0.000 & 0.0 & 0.000 & 0.00 & 21.500 & 28.9 & 27.327 & 21.500 & 28.9 \\
\hline Copper & 1.252 & 0.000 & 0.0 & 0.00 & 0.000 & 0.0 & 0.000 & 0.00 & 222.000 & 298.7 & 277.944 & 222.000 & 298.7 \\
\hline |ron & 1.382 & 0.000 & 0.0 & 0.00 & 0.000 & 0.0 & 0.000 & 0.00 & 92400.000 & 124330.4 & 127696.800 & 92400.000 & 124330.4 \\
\hline Lead & 1.077 & 0.000 & 0.0 & 0.00 & 0.000 & 0.0 & 0.000 & 0.00 & 5040.000 & 6781.7 & 5428.080 & 5040.000 & 6781.7 \\
\hline Magnesium & 1.658 & 0.000 & 0.0 & 0.00 & 0.000 & 0.0 & 0.000 & 000 & 486.000 & 653.9 & 805.788 & 486.000 & 653.9 \\
\hline Manganese & 1.582 & 0.000 & 00 & 0.00 & 0.000 & 0.0 & 0.000 & 0.00 & 2900.000 & 3902.1 & 4587.800 & 2900.000 & 3902.1 \\
\hline Nickel & 1.272 & 0.000 & 0.0 & 0.00 & 0.000 & 0.0 & 0.000 & 0.00 & 5200.000 & 6997.0 & 6614.400 & 5200.000 & 6997.0 \\
\hline Phosphate & 1.000 & 0.000 & 0.0 & 0.00 & 0.000 & 0.0 & 0.000 & 0.00 & 109500.000 & 147339.6 & 109500.000 & 109500.000 & 147339.6 \\
\hline Polassium & 1.000 & 0.000 & 0.0 & 0.00 & 0.000 & 0.0 & 0.000 & 0.00 & 730.000 & 982.3 & 730.000 & 730.000 & 982.3 \\
\hline Silicon & 2.139 & 0.000 & 0.0 & 0.00 & 0.000 & 0.0 & 0.000 & 0.00 & 18100.000 & 24354.8 & 38715.900 & 18100.000 & 24354.8 \\
\hline Silver & 1.000 & 0.000 & 0.0 & 0.00 & 0.000 & 0.0 & 0.000 & 0.00 & 581.000 & 781.8 & 581.000 & 581.000 & 781.8 \\
\hline Sodium & 1.000 & 0.000 & 0.0 & 0.00 & 0.000 & 0.0 & 0.000 & 0.00 & 141000.000 & 189725.0 & 141000.000 & 141000.000 & 1897250 \\
\hline Strontium & 1.183 & 0.000 & 0.0 & 0.00 & 0.000 & 0.0 & 0.000 & 0.00 & 232.000 & 312.2 & 274.456 & 232.000 & 312.2 \\
\hline Zinc & 1.245 & 0.000 & 0.0 & 0.00 & 0.000 & 0.0 & 0,000 & 0.00 & 375.000 & 504.6 & 466.875 & 375.000 & 504.6 \\
\hline Zirconium & 1.351 & 0.000 & 0.0 & 0.00 & 0.000 & 0.0 & 0000 & 0.00 & 2600.000 & 3498.5 & 3512.600 & 2600.000 & 3498.5 \\
\hline Uranium & $\$ .134$ & 0.000 & 0.0 & 0.00 & 0.000 & 0.0 & 0.000 & 0.00 & 23500.000 & 0.0 & 26649.000 & 23500000 & 31620.8 \\
\hline Nitrate & 1.000 & 0.000 & 0.0 & 0.00 & 0.000 & 0.0 & 0.000 & 0.00 & 116870.000 & 157256.5 & 116870.000 & 116870.000 & 157256.5 \\
\hline Chloride & 1.000 & 0.000 & 0.0 & \#DN/O! & 0.000 & 0.0 & 0.000 & $\# \mathrm{D} \mid \mathrm{V} / \mathrm{l} !$ & 0.000 & 0.0 & 0.000 & 0.000 & 0.0 \\
\hline Fluoride & 1.000 & 0.000 & 0.0 & 0.00 & 0.000 & 0.0 & 0.000 & 0.00 & 9800.000 & 13186.6 & 9800.000 & 9800.000 & 13186.6 \\
\hline Free $\mathrm{OH}$ & 1.000 & 0.000 & 0.0 & HDIV/O! & 0.000 & 0.0 & 0.000 & HDIVfo! & 0.000 & 0.0 & 0.000 & 0.000 & 0.0 \\
\hline Carbonate & 1.000 & 0.000 & 0.0 & \#DIVIo! & 0.000 & 0.0 & 0.000 & \#DIVIo! & 0.000 & 0.0 & 0.000 & 0.000 & 0.0 \\
\hline toc & 1.336 & 0.000 & 0.0 & \#DIV/O! & 0.000 & 0.0 & 0.000 & HIVIo! & 0.000 & 0.0 & 0.000 & 0.000 & 0.0 \\
\hline Nitrite & 1.000 & 0.000 & 0.0 & $\#$ DIV/V! & 0.000 & 0.0 & 0.000 & \#DIV/O! & 0.000 & 0.0 & 0.000 & 0.000 & 0.0 \\
\hline Sulfate & 1.000 & 0.000 & 0.0 & 0.00 & 0.000 & 0.0 & 0.000 & 0.00 & 13000.000 & 17492.4 & 13000.000 & 13000.000 & 17492.4 \\
\hline \multicolumn{2}{|l|}{ Total } & $\begin{array}{r}0.000 \\
0.00 \\
\end{array}$ & \multicolumn{2}{|l|}{$\begin{array}{l}\text { \%total solids } \\
\text { in wash }\end{array}$} & & & $\begin{array}{r}0.000 \\
0.00\end{array}$ & $\begin{array}{l}\% \text { total solids } \\
\text { in acid }\end{array}$ & & & $\begin{array}{c}923687.209 \\
92.37 \begin{array}{l}\% \text { total solids } \\
\text { in fusion }\end{array}\end{array}$ & \multicolumn{2}{|r|}{$\begin{array}{l}\text { mass } \\
\text { balance }\end{array}$} \\
\hline \multicolumn{2}{|l|}{ Radionuclides } & uCilg & Ci/tank & dissolved & $\mathrm{uCV} / \mathrm{g}$ & Ci/tank & & dissolved & $\mathrm{uCi/g}$ & Ci/tank & & uCi/g & Ciltank \\
\hline \multicolumn{2}{|l|}{$\overline{\mathrm{P}} \mathrm{U}-239,240$} & 0.000 & 0.0 & 0.00 & & 0.0 & & 0.00 & 2.900 & 3902.1 & & 2.900 & 3902.1 \\
\hline \multicolumn{2}{|l|}{ C.14 } & 0.000 & 0.0 & \#DIVIO! & & 0.0 & & HDIVIOI & 0.000 & 0.0 & & 0.000 & 0.0 \\
\hline \multicolumn{2}{|l|}{ Sr-90 } & 0.000 & 0.0 & 0.00 & & 0.0 & & 0.00 & 5500.000 & 7400621.3 & & 5500.000 & 7400621.3 \\
\hline \multicolumn{2}{|l|}{ TC-99 } & 0.000 & 0.0 & \#D|V/O! & & 0.0 & & \#DIV/O! & $<0.54$ & 0.0 & & 0.000 & 0.0 \\
\hline \multicolumn{2}{|l|}{$A m-241$} & 0.000 & 0.0 & 0.00 & & 0.0 & & 0.00 & 1.140 & 1533.9 & & $\$ .140$ & 1533.9 \\
\hline \multicolumn{2}{|l|}{ Co-60 } & 0.000 & 0.0 & \#DIVIO! & & 0.0 & & \#DIV/O! & 0.000 & 0.0 & & 0.000 & 0.0 \\
\hline \multicolumn{2}{|l|}{ Cs-137 } & 0.000 & 0.0 & 0.00 & & 0.0 & & 0.00 & 75.400 & 101455.8 & & 75.400 & 101455.8 \\
\hline \multicolumn{2}{|l|}{$I-129$} & 0.000 & 0.0 & HDIVIO! & & 0.0 & & \#DIVIO! & 0.000 & 0.0 & & 0.000 & 0.0 \\
\hline
\end{tabular}




\section{C-108 [TBP(f), 1C]}

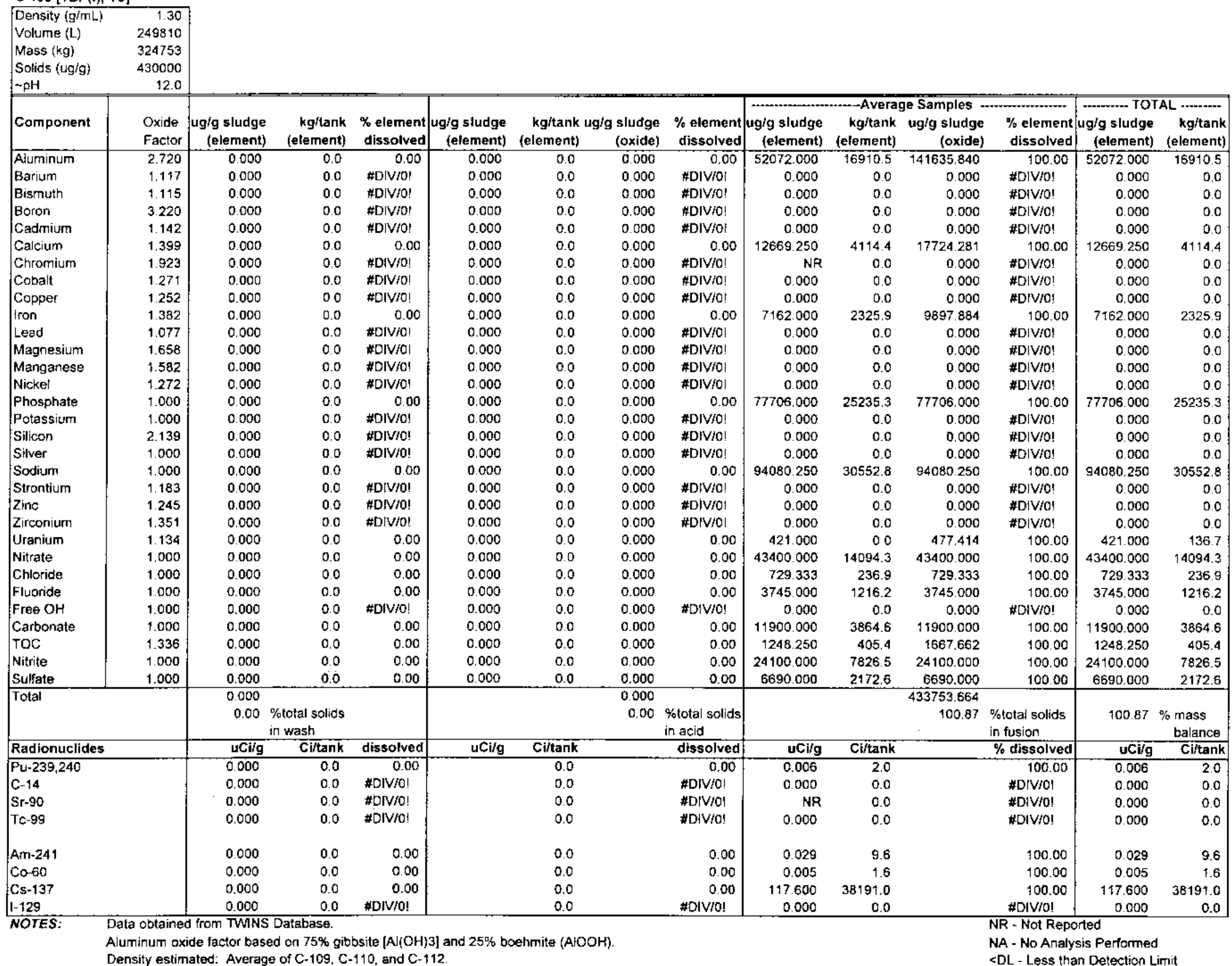




\begin{tabular}{|c|c|c|c|c|c|c|c|c|c|c|c|c|c|c|}
\hline \multicolumn{2}{|c|}{ S-10S (REDOX, EB) } & & & & & & & & & & & & & \\
\hline Density $(\mathrm{g} / \mathrm{mL})$ & 170 & & & & & & & & & & & & & \\
\hline Volume (L) & 1718390 & & & & & & & & & & & & & \\
\hline Mass $(\mathrm{kg})$ & 2921263 & & & & & & & & & & & & & \\
\hline Solids (ug/g) & 966000 & & & & & & & & & & & & & \\
\hline$-\mathrm{pH}$ & 13.0 & & & & & & & & & & & & & \\
\hline & & |-..............-.... & --- WATER .... & 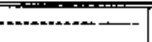 & 7 & - RESIDUALL & SOtIDS .... & (2+........ & & & & & - TOTA & $\overline{A L} \ldots$ \\
\hline Component & $\begin{array}{l}\text { Oxide } \\
\text { Factor }\end{array}$ & $\begin{array}{r}\text { ug/g sludge } \\
\text { (element) }\end{array}$ & $\begin{array}{r}k g / \tan k \\
(\text { (element) }\end{array}$ & $\begin{array}{c}\% \text { element } \\
\text { dissolved }\end{array}$ & $\begin{array}{r}\text { ogig sludge } \\
\text { (element) }\end{array}$ & $\begin{array}{r}\text { kg/tank } \\
\text { (element) }\end{array}$ & $\begin{array}{r}\text { ug/g sludge } \\
\text { (oxide) }\end{array}$ & $\begin{array}{l}\text { \% element } \\
\text { dissolved }\end{array}$ & $\begin{array}{r}\text { ug/g sludge } \\
\text { (element) }\end{array}$ & $\begin{array}{r}\text { kg/tank } \\
\text { (element) }\end{array}$ & $\begin{array}{r}\text { ug/g sludge } \\
\text { (oxide) }\end{array}$ & $\begin{array}{r}\% \text { element } \\
\text { dissolved }\end{array}$ & $\begin{array}{r}\text { ug/g sludge } \\
\text { (element) }\end{array}$ & $\begin{array}{r}\mathrm{kg} / \operatorname{tank} \\
(\mathrm{elem} \text { ent }\end{array}$ \\
\hline Aluminum & 1.889 & 6694.380 & 19556.0 & 97.71 & 156.650 & 457.6 & 295.912 & 2.29 & 0.000 & 0.0 & 0.000 & 0.00 & 6851.030 & 20013.7 \\
\hline Barium & 1.117 & 0.000 & 0.0 & \#DIVIo! & 0.000 & 0.0 & 0.000 & \#DIVIo! & 0.000 & 00 & 0.000 & \#DIV/O! & 0.000 & 0.0 \\
\hline Bismuth & 1.115 & 0.000 & 0.0 & \#DIVIO! & 0.000 & 0.0 & 0000 & \#DIVIO! & 0.000 & 0.0 & 0.000 & \#DIVI0! & 0.000 & 0.0 \\
\hline Boron & 3.220 & 0.000 & 0.0 & \#DIV/OI & 0.000 & 0.0 & 0.000 & \#DIVIo! & 0.000 & 0.0 & 0.000 & \#DIVIo! & 0.000 & 0.0 \\
\hline Cadmium & 1.142 & 0.000 & 0.0 & \#DIVIo! & 0.000 & 0.0 & 0.000 & \#DIV/o! & 0.000 & 0.0 & 0.000 & \#DIV/O! & 0.000 & 0.0 \\
\hline Calcium & 1.399 & 0.000 & 0.0 & \#DIVIOI & 0.000 & 0.0 & 0.000 & \#DIVIO! & 0.000 & 0.0 & 0.000 & \#DIVfo! & 0.000 & 0.0 \\
\hline Chromium & 1.923 & 1841.840 & 5380.5 & 100.00 & NA & 0.0 & 0.000 & 0.00 & 0.000 & 0.0 & 0.000 & 0.00 & 1841.840 & 5380.5 \\
\hline Cobalt & 1.271 & 0.000 & 0.0 & HDIVIO! & 0.000 & 0.0 & 0.000 & \#DIVio! & 0.000 & 0.0 & 0.000 & HDIVIO! & 0.000 & 0.0 \\
\hline Copper & 1.252 & 0.000 & 0.0 & \#DIVIO! & 0.000 & 0.0 & 0.000 & \#DIVIO! & 0.000 & 0.0 & 0.000 & \#DIVfo1 & 0.000 & 0.0 \\
\hline Iron & 1.382 & 8.753 & 25.6 & 100.00 & NA & 0.0 & 0.000 & 0.00 & 0.000 & 0.0 & 0.000 & 0.00 & 8.753 & 25.6 \\
\hline Lead & 1.077 & 0.000 & 0.0 & \#DIVIO! & 0.000 & 0.0 & 0.000 & \#DIVIo! & 0.000 & 0.0 & 0.000 & \#DIVIO! & 0.000 & 0.0 \\
\hline Magnesium & 1.658 & 5.222 & 15.3 & 100.00 & 0.000 & 0.0 & 0.000 & 0.00 & 0.000 & 0.0 & 0.000 & 0.00 & 5.222 & t5.3. \\
\hline Manganese & 1.582 & 0.000 & 0.0 & 0.00 & 128.380 & 375.0 & 203.097 & 100.00 & 0.000 & 0.0 & 0.000 & 0.00 & 128.380 & 375.0 \\
\hline Nickel & $\{.272$ & 0.000 & 0.0 & HDIVIO! & 0.000 & 0.0 & 0.000 & \#DIV\%! & 0.000 & 0.0 & 0.000 & \#DIVIO! & 0.000 & 0.0 \\
\hline Phosphate & 1.000 & 6489.450 & 18957.4 & 100.00 & 0.000 & 0.0 & 0.000 & 0.00 & 0.000 & 0.0 & 0.000 & 0.00 & 6489.450 & 18957.4 \\
\hline Potassium & 1.000 & 0.000 & 00 & \#DIVIO! & 0000 & 0.0 & 0.000 & \#DIV/O! & 0.000 & 0.0 & 0.000 & \#DIV/0! & 0.000 & 0.0 \\
\hline Silicon & 2.139 & 64.464 & 188.3 & 100.00 & NA & 0.0 & 0.000 & 0.00 & 0.000 & 0.0 & 0.000 & 0.00 & 64.464 & 188.3 \\
\hline Unaccounted & 1.000 & & 0.0 & 0.00 & 7000.000 & 20448.8 & 7000.000 & 100.00 & 0.000 & 00 & 0.000 & 0.00 & 7000.000 & 20448.8 \\
\hline Sodium & 1.000 & 284549.100 & 831242.8 & 99.61 & $11 \uparrow 3.500$ & 3252.8 & 1113.500 & 0.39 & 0.000 & 0.0 & 0.000 & 0.00 & 285662600 & 834495.6 \\
\hline Strontium & 1.183 & 13.298 & 38.8 & 100.00 & 0.000 & 0.0 & 0.000 & 0.00 & 0.000 & 0.0 & 0.000 & 0.00 & 13.298 & 38.8 \\
\hline Zinc & 1.245 & 61.221 & 178.8 & 100.00 & 0.000 & 0.0 & 0.000 & 0.00 & 0.000 & 0.0 & 0.000 & 0.00 & 61.221 & 178.8 \\
\hline Zirconium & 1.351 & 0.000 & 0.0 & \#DIVIO! & 0.000 & 0.0 & 0.000 & \#DIV/O! & 0.000 & 0.0 & 0.000 & HDIVIO! & 0.000 & 0.0 \\
\hline Uranium & 1.134 & 0.000 & 0.0 & \#DIVIo! & NA & 0.0 & 0.000 & \#DIV/O! & 0.000 & 0.0 & 0.000 & \#D|V/o! & 0.000 & 0.0 \\
\hline Nitrate & 1.000 & 552891.200 & 1615140.6 & 100,00 & 0.000 & 0.0 & 0.000 & 0.00 & 0.000 & 0.0 & 0.000 & 0.00 & 552891.200 & 1615140.6 \\
\hline Chloride & 1.000 & 0.000 & 0.0 & \#DIVIOI & 0.000 & 0.0 & 0.000 & \#DIV/O! & 0.000 & 0.0 & 0.000 & \#DIVio! & 0.000 & 0.0 \\
\hline Fluoride & 1000 & 0.000 & 0.0 & \#DIVIO! & 0.000 & 0.0 & 0.000 & \#DIV/OI & 0.000 & 0.0 & 0.000 & \#DIVio! & 0.000 & 0.0 \\
\hline Free $\mathrm{OH}$ & 1.000 & 8602.000 & 25128.7 & 100.00 & 0.000 & 0.0 & 0.000 & 0.00 & 0.000 & 0.0 & 0.000 & 0.00 & 8602.000 & 25128.7 \\
\hline Carbonate & 1.000 & 57684.000 & $168510 . \uparrow$ & 100.00 & 0.000 & 0.0 & 0.000 & 0.00 & 0.000 & 0.0 & 0.000 & 0.00 & 57684.000 & 168510.1 \\
\hline TOC & 1.336 & 0.000 & 0.0 & \#DIVIO! & 0.000 & 0.0 & 0.000 & \#DIV/0! & 0.000 & 0.0 & 0.000 & HDIVIO! & 0.000 & 0.0 \\
\hline Nitrite & 1.000 & 23276.000 & 67995.3 & 100.00 & 0.000 & 0.0 & 0.000 & 0.00 & 0.000 & 0.0 & 0.000 & 0.00 & 23276000 & 67995.3 \\
\hline Sulfate & 1.000 & NR & 0.0 & \#DIVfo! & 0.000 & 0.0 & 0.000 & \#DIV/OI & 0.000 & 0.0 & 0.000 & \#DIVIO! & 0.000 & 0.0 \\
\hline Total & & $\begin{array}{r}942180.928 \\
97.53\end{array}$ & $\begin{array}{l}\% \text { total solids } \\
\text { in wash }\end{array}$ & & & & $\begin{array}{r}8612.509 \\
0.89\end{array}$ & $\begin{array}{l}\% \text { residual } \\
\text { solids }\end{array}$ & & & $\begin{array}{r}0.000 \\
0.00\end{array}$ & & 98.43 & $\begin{array}{l}\% \text { mass } \\
\text { balance }\end{array}$ \\
\hline Radionuclides & & uCi/g & Ci/tank & dissolved & uCV/g & Ci/tank & & dissolved & uCi/g & Ci/tank & & dissolved & $\mathrm{uCi} / \mathrm{g}$ & Ciltank \\
\hline$\overline{\mathrm{P} U}-239,240$ & & 0.000 & 0.0 & \#DIV/O] & NA & 0.0 & & FDIV/o! & 0.000 & 0.0 & & \#DIVIO! & 0.000 & 0.0 \\
\hline C-14 & & 0.000 & 0.0 & \#DIVIO! & NA & 0.0 & & \#DIVIO! & 0.000 & 0.0 & & \#DIVIO! & 0.000 & 0.0 \\
\hline Sr-9o & & 0.058 & 169.2 & 0.06 & 97.000 & 283362.5 & & 99.94 & 0.000 & 0.0 & & 0.00 & 97.058 & 283531.8 \\
\hline Tc-99 & & 0.024 & 68.7 & 73.23 & 0.009 & 25.1 & & 26.77 & 0.000 & 0.0 & & 0.00 & 0.032 & 93.9 \\
\hline Am-241 & & 0.000 & 0.0 & \#DIV/O! & NA & 0.0 & & \#DIV/OI & 0.000 & 0.0 & & \#DIVio! & 0.000 & 0.0 \\
\hline Co-60 & & 0.000 & 0.0 & 0.00 & 9.000 & 26291.4 & & 100.00 & 0.000 & 0.0 & & 0.00 & 9.000 & 26291.4 \\
\hline Cs-137 & & 38.203 & 111601.0 & 67.97 & 18.000 & 52582.7 & & 32.03 & 0.000 & 0.0 & & 0.00 & 56.203 & 164183.7 \\
\hline $1-129$ & & 0.000 & 0.0 & \#DIV/0! & NA & 0.0 & & HDIVIO! & 0.000 & 0.0 & & \#DIV/o! & 0.000 & 0.0 \\
\hline NOTES: & Percent wat & er based on 19 & 974 sample. & & & & & & & & & $\sqrt{R}-$ Not R & & \\
\hline & $\begin{array}{l}\text { Nitrate adjus } \\
\text { Unaccounte }\end{array}$ & $\begin{array}{l}\text { sted from } 615,0 \\
\text { d solids added }\end{array}$ & $\begin{array}{l}000 \mathrm{ug} / \mathrm{g} \text { to } 5 \\
\text { to adjust res }\end{array}$ & & unti & & & & & & & $=\mathrm{DL}-$ Les & $\begin{array}{l}\text { formed } \\
\text { ction Li }\end{array}$ & 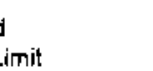 \\
\hline
\end{tabular}




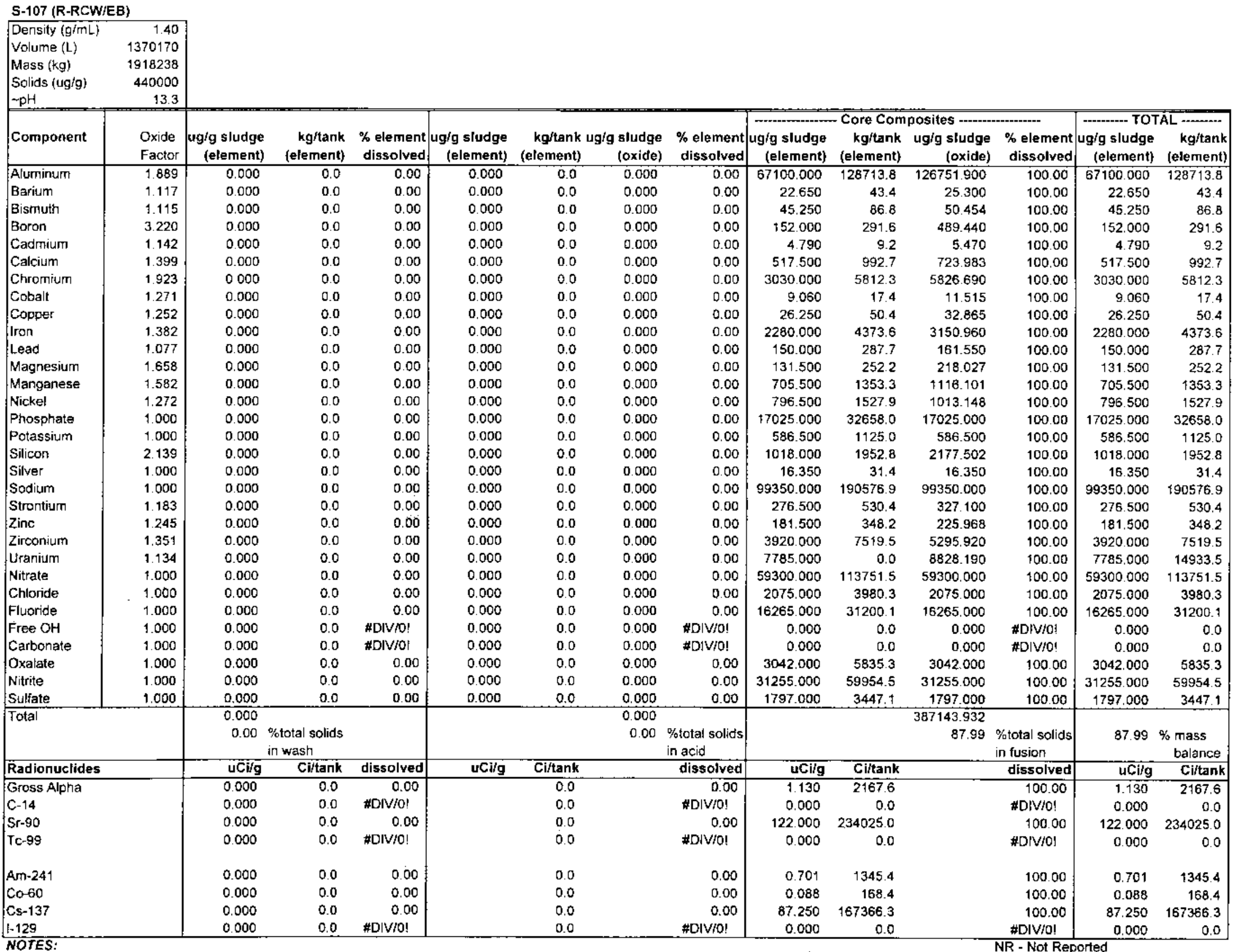

NOTES:

Analyses obtained from TWNS Database. Percent water obtained from historical samples (1980). 


\begin{tabular}{|c|c|c|c|c|c|c|c|c|c|c|c|c|c|c|}
\hline \multicolumn{15}{|c|}{ S-109 (REDOX, EB) } \\
\hline Density $(\mathrm{g} / \mathrm{mL})$ & 1.70 & & & & & & & & & & & & & \\
\hline Volume (L) & 2149880 & & & & & & & & & & & & & \\
\hline Mass (kg) & 3654796 & & & & & & & & & & & & & \\
\hline Solids (ug/g) & 989400 & & & & & & & & & & & & & \\
\hline$\sim \mathrm{pH}$ & 13.0 & & & & & & & & & & & & & \\
\hline \multirow{3}{*}{ Component } & \multirow{3}{*}{$\begin{array}{l}\text { Oxide } \\
\text { Factor }\end{array}$} & \multirow{3}{*}{$\begin{array}{c}\text { ugig sludge } \\
\text { (element) }\end{array}$} & \multirow{3}{*}{$\begin{array}{r}\text { kg/tank } \\
\text { (element) }\end{array}$} & \multirow{3}{*}{$\begin{array}{l}\text { \% element } \\
\text { dissolved }\end{array}$} & \multirow{3}{*}{$\begin{array}{r}\text { ug/g sludge } \\
\text { (element) }\end{array}$} & \multirow{3}{*}{\multicolumn{2}{|c|}{$\begin{array}{c}\text { RESIDUAL SOLIDS -- } \\
\text { kg/tank ug/g sludge } \\
\text { (element) } \\
\text { (oxide) }\end{array}$}} & \multirow{3}{*}{$\begin{array}{c}\text { \% element } \\
\text { dissolved }\end{array}$} & \multirow{3}{*}{$\begin{array}{r}u g / g \text { sludge } \\
\text { (element) }\end{array}$} & \multirow{3}{*}{\multicolumn{2}{|c|}{$\begin{array}{r}\text { kg/tank ug/g sludge } \\
\text { (element) } \quad \text { (oxide) }\end{array}$}} & \multirow{3}{*}{$\begin{array}{l}\text { \% element } \\
\text { dissolved }\end{array}$} & \multirow{3}{*}{$\begin{array}{l}\text { ug/g sludge } \\
\text { (element) }\end{array}$} & \multirow{3}{*}{$\begin{array}{r}\mathrm{kg} / \operatorname{tank} \\
\text { (element) }\end{array}$} \\
\hline & & & & & & & & & & & & & & \\
\hline & & & & & & & & & & & & & & \\
\hline Aluminum & 1.889 & 8316.000 & 30393.3 & 89.47 & 978.900 & 3577.7 & 1849.142 & 10.53 & 0.000 & 0.0 & 0.000 & 0.00 & 9294.900 & 33971.0 \\
\hline Barium & 1.117 & 0.000 & 0.0 & \#DIV/O! & 0.000 & 0.0 & 0.000 & \#DIV/o! & 0.000 & 0.0 & 0.000 & \#DIV/o! & 0.000 & 0.0 \\
\hline Bismuth & 1.115 & 0.000 & 0.0 & HDIVIO! & 0.000 & 0.0 & 0.000 & \#DIV/O! & 0.000 & 0.0 & 0.000 & HDIV/o! & 0.000 & 00 \\
\hline Boron & 3.220 & 0.000 & 0.0 & $\#$ DIV/O! & 0.000 & 0.0 & 0.000 & \#DIV/o! & 0.000 & 0.0 & 0.000 & \#DIV/o! & 0.000 & 0.0 \\
\hline Cadmium & 1.142 & 0.000 & 0.0 & HDIV/0! & 0.000 & 0.0 & 0.000 & WIVIO! & 0.000 & 0.0 & 0.000 & HOIVIo! & 0.000 & 0.0 \\
\hline Calcium & 1.399 & 0.000 & 0.0 & \#DIV/0! & 0.000 & 0.0 & 0.000 & \#DIV/o! & 0.000 & 0.0 & 0.000 & \#DIV/0! & 0.000 & 0.0 \\
\hline Chromium & 1.923 & 2288.000 & 8362.2 & 78.68 & 620.100 & 2266.3 & 1192.452 & 21.32 & 0.000 & 0.0 & 0.000 & 0.00 & 2908.100 & 10628.5 \\
\hline Cobalt & 1.271 & 0.000 & 0.0 & HDIV/0! & 0.000 & 0.0 & 0.000 & HDIV/o! & 0.000 & 0.0 & 0.000 & \#DIV/O! & 0.000 & 0.0 \\
\hline Copper & 1.252 & 0.000 & 0.0 & \#DIVIO! & 0.000 & 0.0 & 0.000 & \#DIV/o! & 0.000 & 0.0 & 0.000 & $\#$ \#IVI0! & 0.000 & 0.0 \\
\hline Iron & 1.382 & 0.000 & 0.0 & 0.00 & 2928.900 & 10704.5 & 4047.740 & 100.00 & 0.000 & 0.0 & 0.000 & 0.00 & 2928.900 & 10704.5 \\
\hline Lead & 1.077 & 0.000 & 0.0 & \#DIV/o! & 0.000 & 0.0 & 0.000 & \#DIV/o! & 0.000 & 0.0 & 0.000 & \#DIV/o! & 0.000 & 0.0 \\
\hline Magnesium & 1.658 & 0.000 & 0.0 & \#DIVIO! & 0.000 & 0.0 & 0.000 & \#DIV/o! & 0.000 & 0.0 & 0.000 & \#DIV1V! & 0.000 & 00 \\
\hline Manganese & 1.582 & 0.000 & 0.0 & 0.00 & 210.600 & 769.7 & 333.469 & 100.00 & 0.000 & 0.0 & 0.000 & 0.00 & 210.600 & 769.7 \\
\hline Nickel & 1.272 & 7.748 & 28.3 & 100.00 & 0.000 & 0.0 & 0.000 & 0.00 & 0.000 & 0.0 & 0.000 & 0.00 & 7.748 & 28.3 \\
\hline Phosphate & 1.000 & LT & 0.0 & \#DIVIO! & 0.000 & 0.0 & 0.000 & \#DIV/0! & 0.000 & 0.0 & 0.000 & $\# \mathrm{D} \mid \mathrm{V} / 0 \mathrm{~S}$ & 0.000 & 0.0 \\
\hline Potassium & 1.000 & 0.000 & 0.0 & HDIVIOF & 0.000 & 0.0 & 0.000 & \#DIV/o! & 0.000 & 0.0 & 0.000 & $\# D \mid V / O$ ! & 0.000 & 0.0 \\
\hline Silicon & 2.139 & 55.440 & 202.6 & 8.46 & 549.900 & 2009.8 & 1176.236 & 90.84 & 0.000 & 0.0 & 0.000 & 0.00 & 605.340 & 2212.4 \\
\hline Unaccounted & 1.000 & 0000 & 0.0 & 0.00 & 25000.000 & 91369.9 & 25000.000 & 100.00 & 0.000 & 0.0 & 0.000 & 0.00 & 25000.000 & 913699 \\
\hline Sodium & 1.000 & 265650.000 & 970896.6 & 98.70 & 5148.000 & 18814.9 & 5148.000 & 1.90 & 0.000 & 0.0 & 0.000 & 0.00 & 270798.000 & 989711.4 \\
\hline Strontium & 1.183 & 0.000 & 0.0 & \#DIVIo! & 0.000 & 0.0 & 0.000 & \#DIVIOI & 0.000 & 0.0 & 0.000 & $\# \mathrm{D} \mid \mathrm{V} / 0$ ! & 0.000 & 0.0 \\
\hline Zinc & 1.245 & 0.000 & 0.0 & \#DIVO! & 0.000 & 0.0 & 0.000 & \#DIV/o! & 0.000 & 0.0 & 0.000 & HDV/01 & 0.000 & 0.0 \\
\hline Zirconium & 1.351 & 0.000 & 0.0 & HDIVIOI & 0.000 & 0.0 & 0.000 & HDIV/o! & 0.000 & 0.0 & 0.000 & \#DIV/o! & 0.000 & 0.0 \\
\hline Uranium & 1.134 & 0.000 & 0.0 & 0.00 & 3.237 & 11.8 & 3.671 & 100.00 & 0.000 & 0.0 & 0.000 & 0.00 & 3.237 & 11.8 \\
\hline Nitrate & 1.000 & 549692.000 & 2009012.1 & 100.00 & 0.000 & 0.0 & 0.000 & 0.00 & 0.000 & 0.0 & 0.000 & 0.00 & 549692.000 & 2009012.1 \\
\hline Chloride & 1.000 & 0.000 & 0.0 & \#DIVIOI & 0.000 & 0.0 & 0.000 & \#DIVIo! & 0.000 & 0.0 & 0.000 & \#DIV/0! & 0.000 & 0.0 \\
\hline Fluoride & 1.000 & 0.000 & 0.0 & ADIV/0I & 0.000 & 0.0 & 0.000 & \#DIVIO! & 0.000 & 0.0 & 0.000 & \#OIV/O! & 0.000 & 0.0 \\
\hline Free OH & 9.000 & 13464.000 & 49208.2 & 100.00 & 0.000 & 0.0 & 0.000 & 0.00 & 0.000 & 0.0 & 0.000 & 0.00 & 13464.000 & 49208.2 \\
\hline Carbonate & 1.000 & 36960.000 & 135081.3 & 100.00 & 0.000 & 0.0 & 0.000 & 0.00 & 0.000 & 0.0 & 0.000 & 0.00 & 36960.000 & 135081.3 \\
\hline TOC & 1.336 & 1399.200 & 3827.7 & 100.00 & 0.000 & 0.0 & 0.000 & 0.00 & 0.000 & 0.0 & 0.000 & 0.00 & 1047.305 & 3827.7 \\
\hline Nitrite & 1.000 & LT & 0.0 & AOIVIO! & 0.000 & 0.0 & 0.000 & \#DIVIo! & 0.000 & 0.0 & 0.000 & $\#$ OIV/0! & 0.000 & 0.0 \\
\hline Sulfate & 1.000 & $10+37.600$ & 37050.9 & 100.00 & 0.000 & 0.0 & $0: 000$ & 0.00 & 0.000 & 0.0 & 0.000 & 0.00 & 10137,600 & 37050.9 \\
\hline \multicolumn{2}{|l|}{ Total } & $\begin{array}{r}887969.988 \\
89.75\end{array}$ & \multicolumn{2}{|l|}{$\begin{array}{l}\text { \%total solids } \\
\text { in wash }\end{array}$} & \multicolumn{4}{|c|}{$\begin{aligned} & 38750.410 \\
& 3.92 \% \text { total s } \\
& \text { in acid }\end{aligned}$} & & & $\begin{array}{r}0.000 \\
0.00\end{array}$ & $\begin{array}{l}\text { \%total solids } \\
\text { in fusion }\end{array}$ & 93.66 & $\begin{array}{l}\% \text { mass } \\
\text { balance }\end{array}$ \\
\hline Radionuclides & & $\mathrm{uCi} / \mathrm{g}$ & Ci/tank & dissolved & $\mathrm{uCi} / \mathrm{g}$ & Ci/tank & & dissolved & uCi/g & Ci/tank & & dissolved & $\mathrm{uCi} / \mathrm{g}$ & Ci/tank \\
\hline $\mathrm{Pu}-239,240$ & & $\overline{N R}$ & 0.0 & 0.00 & 0.039 & 142.5 & & 100.00 & 0.000 & 0.0 & & 0.00 & 0.039 & 142.5 \\
\hline$C-14$ & & NR & 0.0 & HDIVIOI & NA & 0.0 & & \#DIV/0I & 0.000 & 0.0 & & \#DIV/O! & 0.000 & 0.0 \\
\hline Sr-90 & & 0.748 & 2733.8 & 0.54 & 137.280 & 501730.4 & & 99.46 & 0.000 & 0.0 & & 0.00 & 138.028 & 504464.2 \\
\hline Tc-99 & & 0.007 & 24.1 & 5.28 & 0.118 & 432.7 & & 94.72 & 0.000 & 0.0 & & 0.00 & 0.125 & 456.9 \\
\hline$A m-24 \uparrow$ & & NR & 0.0 & 0.00 & 0.308 & 1126.0 & & 100.00 & 0.000 & 0.0 & & 0.00 & 0.308 & 1126.0 \\
\hline Co-60 & & 0.002 & 6.2 & 0.49 & 0.343 & 1254.3 & & 99.51 & 0.000 & 0.0 & & 0.00 & 0.345 & 1260.5 \\
\hline Cs-137 & & 83.380 & 304736.9 & 99.95 & 0.043 & 156.8 & & 0.05 & 0.000 & 0.0 & & 0.00 & 83.423 & 304893.7 \\
\hline-129 & & NR & 0.0 & \#DIV/0! & NA & 0.0 & & \#DIVIO! & 0.000 & 0.0 & & \#DIV/o! & 0.000 & 0.0 \\
\hline
\end{tabular}




\begin{tabular}{|c|c|c|c|c|c|c|c|c|c|c|c|c|c|c|}
\hline \multicolumn{15}{|c|}{ S-110 (REDOX, EB) } \\
\hline Density $(\mathrm{g} / \mathrm{mL})$ & 1.70 & & & & & & & & & & & & & \\
\hline Volume (L) & 980315 & & & & & & & & & & & & & \\
\hline Mass $(\mathrm{kg})$ & 1666536 & & & & & & & & & & & & & \\
\hline Solids (ug/g) & 807000 & & & & & & & & & & & & & \\
\hline$-\mathrm{pH}$ & 13.0 & & & & & & & & & & & & & \\
\hline \multirow[b]{2}{*}{ Component } & \multirow[b]{2}{*}{$\begin{array}{l}\text { Oxide } \\
\text { Factor }\end{array}$} & \multirow{2}{*}{$\begin{array}{l}\text { ug/g sludge } \\
\text { (element) }\end{array}$} & \multirow{2}{*}{$\begin{array}{r}\text { WATER } \\
\text { kg/ank } \\
\text { (element) }\end{array}$} & \multirow[b]{2}{*}{$\begin{array}{c}\% \text { element } \\
\text { dissolved }\end{array}$} & \multirow{2}{*}{$\begin{array}{r}\text { ug/g sludge } \\
\text { (element) }\end{array}$} & \multicolumn{3}{|c|}{ RESIDUAL SOLIDS } & \multirow[b]{2}{*}{$\begin{array}{r}\text { ug } / g \text { sludge } \\
\text { (element) }\end{array}$} & \multirow{2}{*}{\multicolumn{2}{|c|}{$\begin{array}{l}\mathrm{kg} / \operatorname{tank} \text { ug/g sludge } \\
\text { (element) } \quad \text { (oxide) }\end{array}$}} & \multirow[b]{2}{*}{$\begin{array}{l}\% \text { element } \\
\text { dissolved }\end{array}$} & \multicolumn{2}{|c|}{ - TOTAL } \\
\hline & & & & & & $\begin{array}{c}\mathrm{kg} / \operatorname{tank} \mathrm{u} \\
\text { (element) }\end{array}$ & $\begin{array}{r}\text { o sludge } \\
\text { (oxide) }\end{array}$ & $\begin{array}{r}\% \text { element } \\
\text { dissolved }\end{array}$ & & & & & $\begin{array}{r}\text { ug/g sludge } \\
\text { (element) }\end{array}$ & $\begin{array}{r}\text { kg/tank } \\
\text { (etement) }\end{array}$ \\
\hline Aluminum & $1 . \overline{889}$ & $7322.400^{\circ}$ & 12203.0 & 100.00 & 0.000 & 0.0 & 0.000 & 0.00 & 0.000 & 0.0 & 0.000 & 0.00 & 7322.400 & 12203.0 \\
\hline Barium & 1.117 & 0.000 & 0.0 & \#DIVIO! & 0.000 & 0.0 & 0.000 & \#DIVIO! & 0.000 & 0.0 & 0.000 & \#DIV/O! & 0.000 & 0.0 \\
\hline Bismuth & 9.115 & 0.000 & 00 & \#DIV/O! & 0.000 & 0.0 & 0.000 & \#DIV/o! & 0000 & 0.0 & 0.000 & \#DIVFo! & 0.000 & 0.0 \\
\hline Boron & 3.220 & 0.000 & 0.0 & \#DIV/o! & 0.000 & 0.0 & 0.000 & \#DIV/0! & 0.000 & 0.0 & 0.000 & \#DIV/O! & 0.000 & 0.0 \\
\hline Cadmium & 1.142 & 0.000 & 0.0 & \#DIV/o! & 0.000 & 0.0 & 0.000 & *DIV/O! & 0.000 & 0.0 & 0.000 & \#DIV/01 & 0.000 & 0.0 \\
\hline Calcium & 1.399 & 0.000 & 0.0 & \#DIV/o! & 0.000 & 0.0 & 0.000 & \#DIVIo! & 0.000 & 0.0 & 0.000 & \#DIV/0! & 0.000 & 0.0 \\
\hline Chromium & 1.923 & 1527.760 & 2546.1 & 100.00 & 0.000 & 0.0 & 0.000 & 0.00 & 0.000 & 0.0 & 0.000 & 0.00 & 1527.760 & 2546.1 \\
\hline Cobalt & 1.271 & 0.000 & 0.0 & \#DIV/0! & 0.000 & 0.0 & 0.000 & \#DIV/o! & 0.000 & 0.0 & 0.000 & \#DIV/o! & 0.000 & 0.0 \\
\hline Copper & 1.252 & 0.000 & 0.0 & \#DIVI0! & 0.000 & 0.0 & 0.000 & \#D|VIO! & 0.000 & 0.0 & 0.000 & \#OIV/o! & 0.000 & 0.0 \\
\hline Iron & 1.382 & 0.000 & 0.0 & \#DIVIO! & 0.000 & 0.0 & 0.000 & HDIV/O! & 0.000 & 0.0 & 0.000 & \#DIV/o! & 0.000 & 0.0 \\
\hline Lead & 1.077 & 0.000 & 0.0 & \#DiVio! & 0.000 & 0.0 & 0.000 & \#DIVIo! & 0.000 & 0.0 & 0.000 & \#DIVio! & 0.000 & 0.0 \\
\hline Magnesium & 1.658 & 0.000 & 0.0 & HDIVIo! & 0.000 & 0.0 & 0.000 & HOIVIO! & 0.000 & 0.0 & 0.000 & \#DIVIO! & 0.000 & 0.0 \\
\hline Manganese & 1.582 & 0.000 & 0.0 & \#DIV/o! & 0.000 & 0.0 & 0.000 & \#DIV/O! & 0.000 & 0.0 & 0.000 & \#DIVIo! & 0.000 & 0.0 \\
\hline Nickel & 1.272 & 9.045 & 15.1 & 100,00 & 0.000 & 0.0 & 0.000 & 0.00 & 0.000 & 0.0 & 0.000 & 0.00 & 9.045 & 15.1 \\
\hline Phosphate & 1.000 & 0.000 & 0.0 & \#DIVIO! & 0.000 & 0.0 & 0.000 & \#DIVIO! & 0.000 & 0.0 & 0.000 & HDIVIO! & 0.000 & 0.0 \\
\hline Potassium & 1.000 & 0.000 & 0.0 & \#DIVIO! & 0.000 & 0.0 & 0.000 & \#DIV/O! & 0.000 & 0.0 & 0.000 & \#DIVIO! & 0.000 & 0.0 \\
\hline Silicon & 2.139 & 69.608 & 116.0 & 100.00 & 0.000 & 0.0 & 0.000 & 0.00 & 0.000 & 0.0 & 0.000 & 0.00 & 69.608 & $\$ 16.0$ \\
\hline Silver & 1.000 & 0.000 & 0.0 & \#DIVIo! & 0.000 & 0.0 & 0.000 & \#DIV/0! & 0.000 & 0.0 & 0.000 & \#DIV/o! & 0.000 & 0.0 \\
\hline Sodium & 1.000 & 251068.400 & 418414.4 & 100.00 & 0.000 & 0.0 & 0.000 & 0.00 & 0.000 & 0.0 & 0.000 & 0.00 & 251068.400 & 418414.4 \\
\hline Strontium & 1.183 & 0.000 & 0.0 & aDIVIol & 0.000 & 0.0 & 0.000 & \#D|V/O! & 0.000 & 0.0 & 0.000 & \#DIV/o! & 0.000 & 0.0 \\
\hline Zinc & 1.245 & 24.973 & 41.6 & 100.00 & 0.000 & 0.0 & 0.000 & 0.00 & 0.000 & 0.0 & 0.000 & 0.00 & 24.973 & 41.6 \\
\hline Zirconium & 1.351 & 0.000 & 0.0 & ADIVIO! & 0.000 & 0.0 & 0.000 & \#DIVID! & 0.000 & 0.0 & 0.000 & \#DIVIO! & 0.000 & 0.0 \\
\hline Uranium & 1.134 & 0.000 & 0.0 & \#DIV/OI & 0.000 & 0.0 & 0.000 & \#DIVIOI & 0.000 & 0.0 & 0.000 & \#DIVfo! & 0.000 & 0.0 \\
\hline Nitrate & 1.000 & 561881.200 & 9363950 & 100.00 & 0.000 & 0.0 & 0.000 & 0.00 & 0.000 & 0.0 & 0.000 & 0.00 & 561881.200 & 936395.0 \\
\hline Chloride & 1.000 & 0.000 & 0.0 & \#DNVO! & 0,000 & 0.0 & 0.000 & \#DIV/OI & 0.000 & 0.0 & 0.000 & \#DIV/0! & 0.000 & 0.0 \\
\hline Fluoride & 1.000 & 0.000 & 0.0 & \#DIV/o! & 0.000 & 0.0 & 0.000 & \#DIV/0! & 0.000 & 0.0 & 0.000 & \#DIV/o! & 0.000 & 0.0 \\
\hline Free $\mathrm{OH}$ & 1.000 & 9605.000 & 16007.1 & 100.00 & 0.000 & 0.0 & 0.000 & 0.00 & 0.000 & 0.0 & 0.000 & 0.00 & 9605.000 & 16007.1 \\
\hline Carbonate & 1.000 & 7458.000 & 12429.0 & 100.00 & 0.000 & 0.0 & 0.000 & 0.00 & 0.000 & 0.0 & 0.000 & 0.00 & 7458.000 & 124290 \\
\hline TOC & 1.336 & 0.000 & 0.0 & ADIVIOI & 0.000 & 0.0 & 0.000 & \#DIVIO! & 0.000 & 0.0 & 0.000 & \#DIVIO! & 0.000 & 0.0 \\
\hline Nitrite & 1.000 & 31188.000 & 51975.9 & 100.00 & 0.000 & 0.0 & 0.000 & 0.00 & 0.000 & 0.0 & 0.000 & 0.00 & 31188.000 & 51975.9 \\
\hline Sulfate & 1.000 & 2625.216 & 4375.0 & 100.00 & 0.000 & 0.0 & 0.000 & 0.00 & 0.000 & 0.0 & 0.000 & 0.00 & 2625.216 & 4375.0 \\
\hline \multirow{2}{*}{\multicolumn{2}{|c|}{ Total }} & 872779.602 & \multirow{2}{*}{\multicolumn{2}{|c|}{$\begin{array}{l}\text { \%total solids } \\
\text { in wash }\end{array}$}} & \multicolumn{4}{|c|}{0.000} & \multicolumn{4}{|c|}{0.000} & \multirow{2}{*}{\multicolumn{2}{|c|}{$\begin{array}{c}108.15 \% \text { mass } \\
\text { balance }\end{array}$}} \\
\hline & & 108.15 & & & & & 0.00 & $\begin{array}{l}\text { \%total solids } \\
\text { in acid }\end{array}$ & & & 0.00 & $\begin{array}{l}\text { \%total solids } \\
\text { in fusion }\end{array}$ & & \\
\hline Radjonuclides & & uCiig & Ci/tank & dissolved & $\mathrm{uCi} / \mathrm{g}$ & Cittank & & dissolved & $\mathrm{uCE} \mathbf{g}$ & Cistank & & dissolved & uCilg & Ci/tank \\
\hline PU-239,240 & & 0.000 & 0.8 & 100.00 & & 0.0 & & 0.00 & 0.000 & 0.0 & & 0.00 & 0.000 & 0.8 \\
\hline$C-14$ & & NR & 0.0 & \#DIVIO! & & 0.0 & & \#DIVIol & 0.000 & 0.0 & & \#DIVIO! & 0.000 & 0.0 \\
\hline Sr-90 & & 1.451 & 2418.0 & 100.00 & & 0.0 & & 0.00 & 0.000 & 0.0 & & 0.00 & 1.451 & 2418.0 \\
\hline Tc-99 & & 0.030 & 49.3 & 100.00 & & 0.0 & & 0.00 & 0.000 & 0.0 & & 0.00 & 0.030 & 49.3 \\
\hline Am-241 & & 0.00002 & 0.0 & 100.00 & & 0.0 & & 0.00 & 0.000 & 0.0 & & 0.00 & 0.000 & 0.0 \\
\hline $\mathrm{Co}-60$ & & LT & 0.0 & \#DIV/OI & & 0.0 & & \#DIV/0! & 0.000 & 0.0 & & \#DIVIo! & 0.000 & 0.0 \\
\hline Cs-137 & & 30.736 & 51222.6 & 100.00 & & 0.0 & & 0.00 & 0.000 & 0.0 & & 0.00 & 30.736 & 51222.6 \\
\hline $1-129$ & & NR & 0.0 & \#DIV/o! & & 0.0 & & \#DIVIo! & 0.000 & 0.0 & & \#DIV/01 & 0.000 & 0.0 \\
\hline
\end{tabular}

NOTES: 
SX-102 (REDOX, EB)

\begin{tabular}{|c|c|c|c|c|c|c|c|c|c|c|c|c|c|c|}
\hline \begin{tabular}{|l|} 
Density (g/mL) \\
Volume (L) \\
Mass (kg) \\
Solids (ug/g) \\
$\sim-\mathrm{pH}$ \\
\end{tabular} & $\begin{array}{r}1.70 \\
2028760 \\
3448892 \\
980000 \\
13.0 \\
\end{array}$ & & & & & & & & & & & & & \\
\hline Component & $\begin{array}{l}\text { Oxide } \\
\text { Factor }\end{array}$ & $\begin{array}{r}\text { ug/g sludge } \\
\text { (element) }\end{array}$ & $\begin{array}{r}\text { kg/tank } \\
\text { (element) }\end{array}$ & $\begin{array}{r}\text { \% element } \\
\text { dissolved }\end{array}$ & $\begin{array}{r}\text { ug/g sludge } \\
\text { (element) }\end{array}$ & $\begin{array}{r}\text { RESIDUALAt s } \\
\text { (element) }\end{array}$ & $\begin{array}{l}\text { SOLIDS -- } \\
\text { ug/g sludge } \\
\text { (oxide) }\end{array}$ & $\begin{array}{c}\% \text { element } \\
\text { dissolved }\end{array}$ & $\begin{array}{r}\text { ug/g sludge } \\
\text { (element) }\end{array}$ & $\begin{array}{c}\mathbf{k g} / \operatorname{tank} \mathrm{u} \\
\text { (element) }\end{array}$ & $\begin{array}{l}9 \text { sludge } \\
\text { (oxide) }\end{array}$ & $\begin{array}{c}\text { \% element } \\
\text { dissolved }\end{array}$ & $\begin{array}{c}\text { ug/g sludge } \\
\text { (element) }\end{array}$ & $\begin{array}{r}\mathrm{kg} / \tan \mathrm{k} \\
\text { (element) }\end{array}$ \\
\hline Afuminum & 1.889 & 6007.500 & 20719.2 & 88.62 & 771.200 & 2659.8 & 1456.797 & 11.38 & 0.000 & 0.0 & 0.000 & 0.00 & 6778.700 & 23379.0 \\
\hline Barium & 1.117 & 0.000 & 0.0 & \#DIVIO! & 0.000 & 0.0 & 0.000 & HDIVIOI & 0.000 & 0.0 & 0.000 & $\#$ \#IV/O! & 0.000 & 0.0 \\
\hline Bismuth & 1.115 & 0000 & 0.0 & \&DIV/O! & 0.000 & 0.0 & 0.000 & \#DIV/O! & 0.000 & 0.0 & 0.000 & \#DIVto! & 0.000 & 0.0 \\
\hline Eoron & 3.220 & 0.000 & 0.0 & \#DIVIO! & 0.000 & 0.0 & 0.000 & HDIV/O! & 0.000 & 0.0 & 0.000 & \#DIV/O! & 0.000 & 0.0 \\
\hline Cadmium & 1.142 & 0.000 & 0.0 & \#DIV/o! & 0.000 & 0.0 & 0.000 & \#DIV/O! & 0.000 & 0.0 & 0.000 & \#DIV/o! & 0.000 & 0.0 \\
\hline Calcium & 1.399 & 15.000 & 51.7 & 100.00 & 0.000 & 0.0 & 0.000 & 0.00 & 0.000 & 0.0 & 0.000 & 0.00 & 15.000 & 51.7 \\
\hline Chromium & 1.923 & 1820.000 & 6277.0 & 59.20 & 1254.400 & 4326.3 & 2412.211 & 40.80 & 0.000 & 0.0 & 0.000 & 0.00 & 3074.400 & 10603.3 \\
\hline Cobalt & 1.271 & 0.000 & 0.0 & \#DIV/o! & 0.000 & 0.0 & 0.000 & \#DIV/O! & 0.000 & 0.0 & 0.000 & \#DIVIO! & 0.000 & 0.0 \\
\hline Copper & 1.252 & 0.000 & 0.0 & HDIV/O! & 0.000 & 0.0 & 0.000 & \#DIV/0! & 0.000 & 0.0 & 0.000 & \#DIVI0! & 0.000 & 0.0 \\
\hline Iron & 1.382 & 0.000 & 0.0 & 0.00 & 1792.000 & 6180.4 & 2476.544 & 100.00 & 0.000 & 0.0 & 0.000 & 0.00 & 1792.000 & 6180.4 \\
\hline Lead & 1.077 & 0.000 & 0.0 & \#DIVIO! & 0.000 & 0.0 & 0.000 & \#DIV/0! & 0.000 & 0.0 & 0.000 & \#DIVI0! & 0.000 & 0.0 \\
\hline Magnesium & 1.658 & 0.000 & 0.0 & HDIVIO! & 0.000 & 0.0 & 0.000 & \#DIV/O! & 0.000 & 0.0 & 0.000 & \#DIV/O! & 0.000 & 0.0 \\
\hline Manganese & 1.582 & 0.000 & 0.0 & 0.00 & 1654.000 & 5704.5 & 2616.628 & 100.00 & 0.000 & 0.0 & 0.000 & 0.00 & 1654.000 & 5704.5 \\
\hline Nickel & 1.272 & 15.950 & 55.0 & 100.00 & 0.000 & 0.0 & 0.000 & 0.00 & 0.000 & 0.0 & 0.000 & 0.00 & 15.950 & 55.0 \\
\hline Phosphate & 1.000 & 1805.000 & 6225.3 & 100.00 & 0.000 & 0.0 & 0.000 & 0.00 & 0.000 & 00 & 0.000 & 0.00 & 1805.000 & 6225.3 \\
\hline Potassium & 1.000 & 0.000 & 0.0 & \#DIVfo! & 0.000 & 0.0 & 0.000 & \#DIVIO! & 0.000 & 0.0 & 0.000 & \#D/V/O! & 0.000 & 0.0 \\
\hline Silicon & 2.139 & 430.500 & 1484.7 & 100.00 & NA & 0.0 & 0.000 & 0.00 & 0.000 & 0.0 & 0.000 & 0.00 & 430.500 & 1484.7 \\
\hline unaccounted & 1.000 & 0.000 & 0.0 & 0.00 & 21500.000 & 74151.2 & 21500.000 & 100.00 & 0.000 & 0.0 & 0.000 & 0.00 & 21500.000 & 74151.2 \\
\hline Sodium & 1.000 & 265175.000 & $9 \nmid 4559.9$ & 09.49 & 1353.600 & 4668.4 & 1353.600 & 0.51 & 0.000 & 0.0 & 0.000 & 0.00 & 268528.600 & 919228.4 \\
\hline Strontium & 1.183 & 0.000 & 0.0 & \#DIVIO! & 0.000 & 0.0 & 0.000 & \#DIVIO! & 0.000 & 0.0 & 0.000 & \#DIVIo! & 0.000 & 0.0 \\
\hline Zinc & 1.245 & 0.000 & 0.0 & HDIVIO! & 0.000 & 0.0 & 0.000 & \#DIV/O! & 0.000 & 0.0 & 0.000 & $\#$ DIVIo! & 0.000 & 0.0 \\
\hline Zirconium & 1.351 & 0.000 & 0.0 & \#DIVIo! & 0.000 & 0.0 & 0.000 & \#DIV/0! & 0.000 & 0.0 & 0.000 & $\#$ \#DIV/0! & 0.000 & 0.0 \\
\hline Uranium & 1.134 & 0.000 & 0.0 & 0.00 & 25.600 & 88.3 & 29.030 & 100.00 & 0.000 & 0.0 & 0.000 & 0.00 & 25.600 & 88.3 \\
\hline Nitrate & 1.000 & 596750.000 & 2058126.3 & 100.00 & 0.000 & 0.0 & 0.000 & 0.00 & 0.000 & 0.0 & 0.000 & 0.00 & 596750.000 & 2058126.3 \\
\hline Chloride & 1.000 & 0.000 & 0.0 & \#DIVIOI & 0.000 & 0.0 & 0.000 & \#DIV/0! & 0.000 & 0.0 & 0.000 & \#DIVfo! & 0.000 & 0.0 \\
\hline Fluoride & 1.000 & 0.000 & 0.0 & \#DIV/0! & 0.000 & 0.0 & 0.000 & HDV/OI! & 0.000 & 0.0 & 0.000 & \#DIVio! & 0.000 & 0.0 \\
\hline Free $\mathrm{OH}$ & 1.000 & 7225.000 & 24918.2 & 100.00 & 0.000 & 0.0 & 0.000 & 0.00 & 0.000 & 0.0 & 0.000 & 0.00 & 7225.000 & 24918.2 \\
\hline Carbonate & 1.000 & 21000.000 & 72426.7 & 100.00 & 0.000 & 0.0 & 0.000 & 0.00 & 0.000 & 0.0 & 0.000 & 0.00 & 21000.000 & 72426.7 \\
\hline TOC & 1.336 & 6300.000 & 16263.5 & 100.00 & 0.000 & 0.0 & 0.000 & 0.00 & 0.000 & 0.0 & 0.000 & 0.00 & 4715.569 & 16263.5 \\
\hline Nitrite & 1.000 & 17250.000 & 59493.4 & 100.00 & 0.000 & 0.0 & 0.000 & 0.00 & 0.000 & 0.0 & 0.000 & 0.00 & 17250.000 & 59493.4 \\
\hline Sulfate & 1.000 & 4800.000 & 16554.7 & 100.00 & 0.000 & 0.0 & 0.000 & 0.00 & 0.000 & 0.0 & 0.000 & 0.00 & 4800.000 & 16554.7 \\
\hline \multicolumn{2}{|l|}{ Total } & $\begin{array}{r}928593.950 \\
94.75 \\
\end{array}$ & \multicolumn{2}{|l|}{$\begin{array}{l}\text { \%total solids } \\
\text { in wash }\end{array}$} & & & $\begin{array}{r}31844.810 \\
3.25\end{array}$ & $\begin{array}{l}\text { \%total solids } \\
\text { in acid }\end{array}$ & & & $\begin{array}{r}0.000 \\
0.00 \\
i\end{array}$ & $\begin{array}{l}\text { \%total solids } \\
\text { in fusion }\end{array}$ & \multicolumn{2}{|c|}{$\begin{array}{l}98.00 \% \text { mass } \\
\text { balance }\end{array}$} \\
\hline \multicolumn{2}{|l|}{ Radionuclides } & uCilg & Ci/tank & dissolved & uCilg & Ci/tank & & dissolved & ucilg & Ci/tank & & dissolved & $\mathrm{uCi} / \mathrm{g}$ & Ci/tank \\
\hline \multicolumn{2}{|l|}{ Pu-239,240 } & 0.001 & 3.5 & 0.86 & 0.118 & 408.3 & & 99.14 & 0.000 & 0.0 & & 0.00 & 0.119 & 411.9 \\
\hline \multicolumn{2}{|l|}{ C-14 } & NR & 0.0 & \#DIV/o! & NA & 0.0 & & \#DIVIo! & 0.000 & 0.0 & & \#OIVIo! & 0.000 & 0.0 \\
\hline \multicolumn{2}{|l|}{ St -90} & 4.550 & 15692.5 & 3.74 & 117.120 & 403934.2 & & 96.26 & 0.000 & 0.0 & & 0.00 & $\{2\} .670$ & $4\{9626.7$ \\
\hline \multicolumn{2}{|l|}{ TC-99 } & 0.033 & 114.7 & 92.60 & 0.003 & 9.2 & & 7.40 & 0.000 & 0.0 & & 0.00 & 0.036 & 123.8 \\
\hline \multicolumn{2}{|l|}{ Am-241 } & 0.001 & 3.5 & 0.01 & 8.640 & 29798.4 & & 99.99 & 0.000 & 0.0 & & 0.00 & 8.641 & 29802.0 \\
\hline \multicolumn{2}{|l|}{ Co.60 } & 0.012 & 41.8 & 100.00 & NA & 0.0 & & 0.00 & 0.000 & 0.0 & & 0.00 & 0.012 & 41.8 \\
\hline \multirow{2}{*}{\multicolumn{2}{|c|}{ Cs-137 }} & 88.500 & 305226.9 & 100.00 & NA & 0.0 & & 0.00 & 0.000 & 0.0 & & 0.00 & 88.500 & 305226.9 \\
\hline & & 0.00001 & 0.0 & 100.00 & NA & 0.0 & & 0.00 & 0.000 & 0.0 & & 0.00 & 0.000 & 0.0 \\
\hline
\end{tabular}

NOTES: Unaccounted solids added to adjust residual solids to $3.2 \%(0.021 \mathrm{~m} /$ sludge/g salt cake "estimated density of sludge, 1.5$)$. 
SX-103 (REDOX, EB)

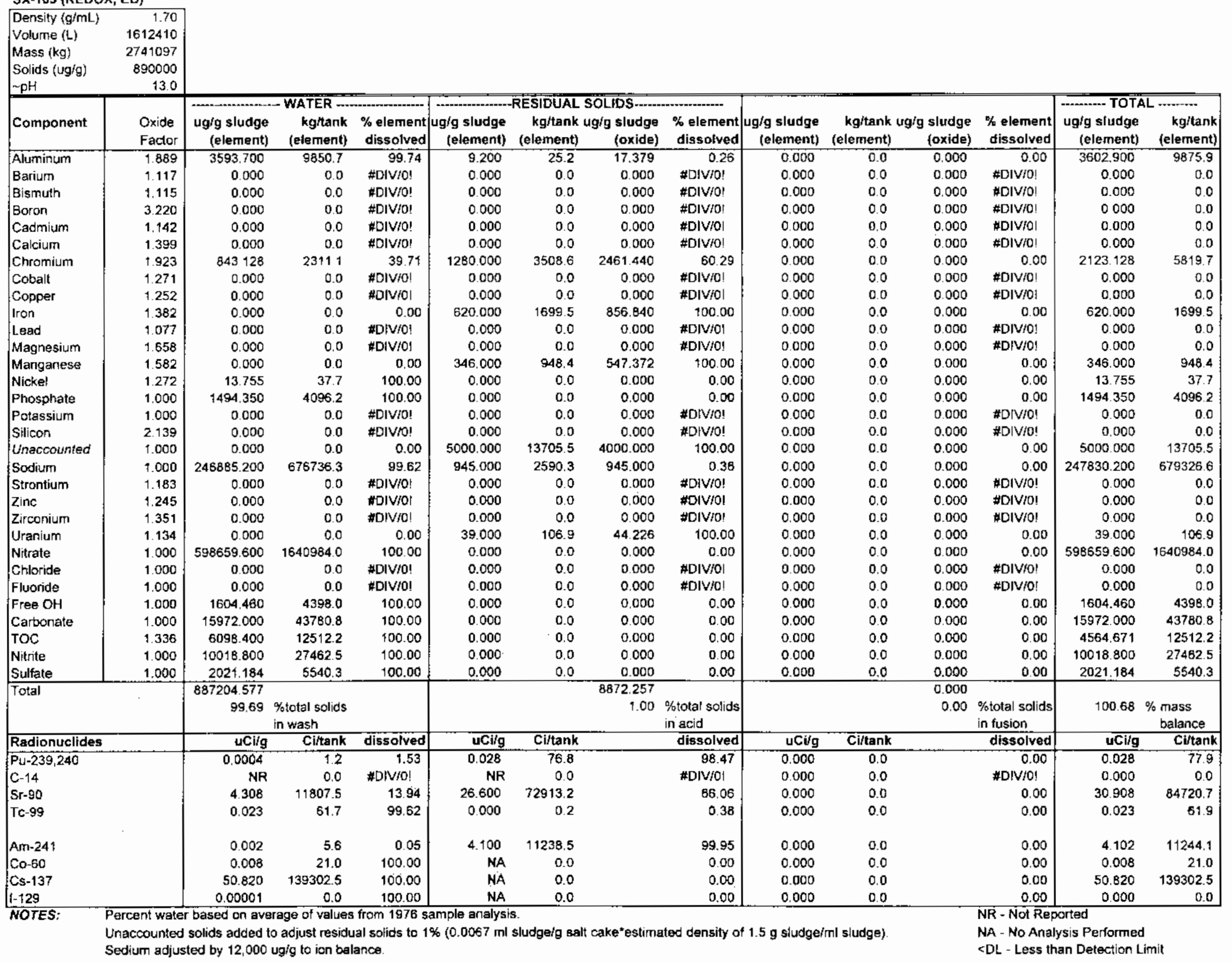


T-105 (BiPO4 1C, CW)

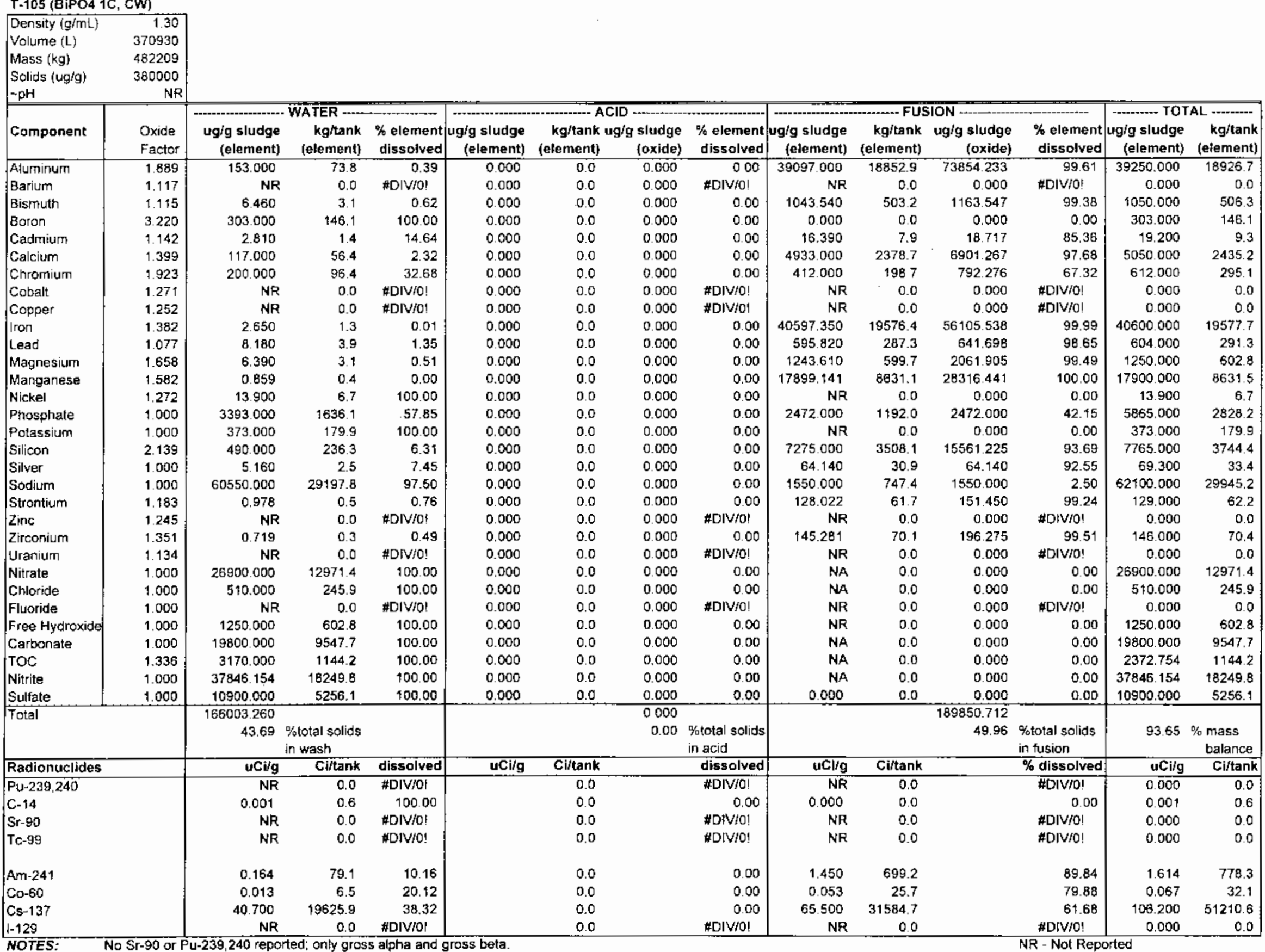

lons in TWINS database reported as ug/mL; assumed ug/g because analysis was performed on a solid. NA - No Analysis Performed 
T.108 (BiPO4 1C/TBP)

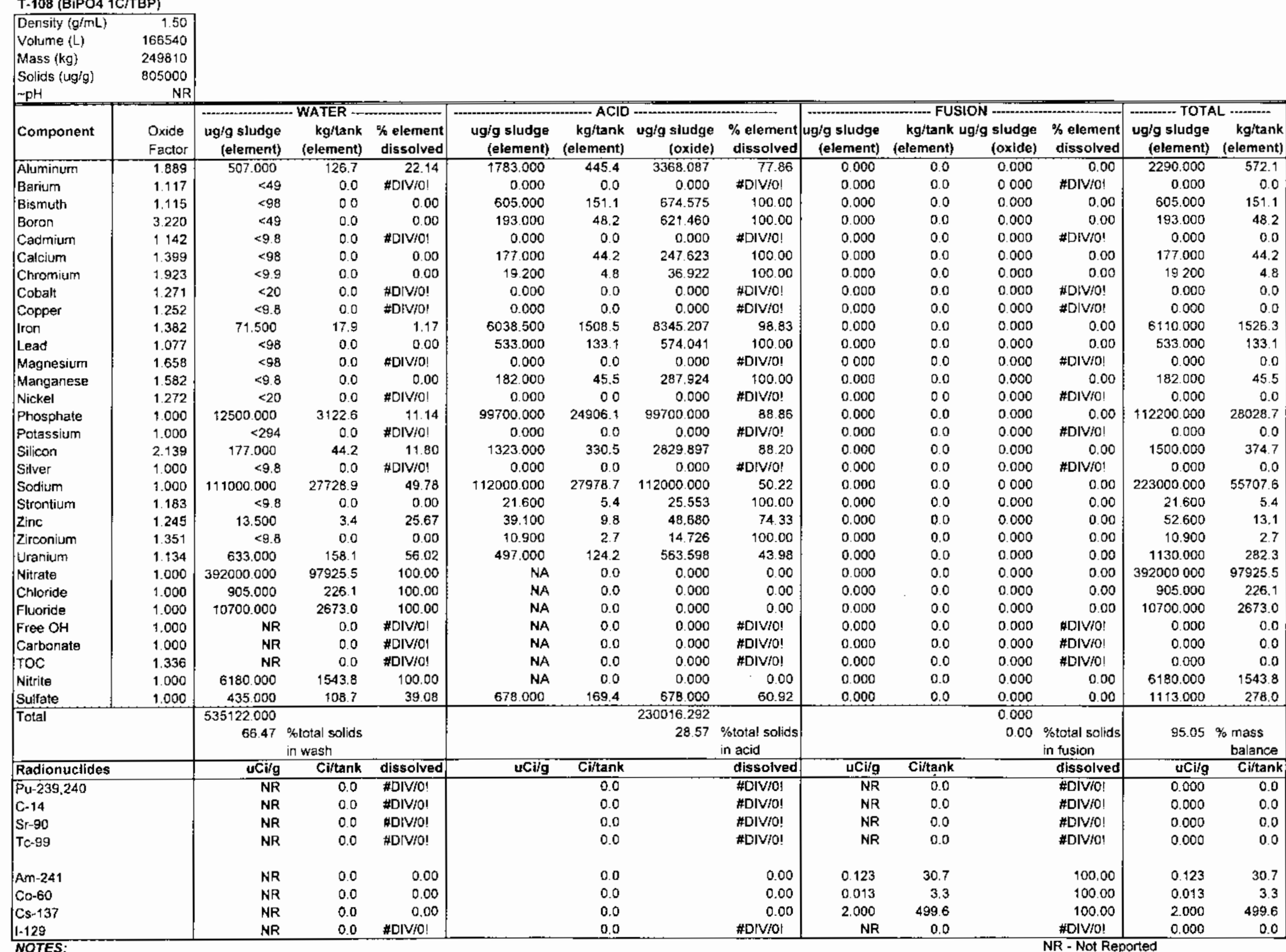

Component concentrations differ by core (vertical inhomogeneity); concentrations refled average of two cores.

NA - No Analysis Performed 
TY-102 (EB, BiPO4 1C)

\begin{tabular}{|c|c|c|c|c|c|c|c|c|c|c|c|c|c|c|}
\hline \begin{tabular}{|l} 
Density (g/mL) \\
Volume (L) \\
Mass (kg) \\
Solids (ug/g) \\
$\sim \mathrm{pH}$ \\
\end{tabular} & $\begin{array}{r}1.70 \\
242240 \\
411808 \\
710000 \\
\mathrm{NR} \\
\end{array}$ & & & & . & & & & & & & & & \\
\hline Component & $\begin{array}{l}\text { Oxide } \\
\text { Factor }\end{array}$ & $\begin{array}{r}\text { ug/g sludge } \\
\text { (element) }\end{array}$ & $\begin{array}{r}\text { WATER -.- } \\
\text { kgitank } \\
\text { (element) }\end{array}$ & $\begin{array}{c}\text { \% element } \\
\text { dissolved }\end{array}$ & $\begin{array}{r}\text { ug/g sludge } \\
\text { (element) }\end{array}$ & $\begin{array}{r}\text { kg/tank u } \\
\text { (element) }\end{array}$ & $\begin{array}{l}\text { OLIDS } \\
\text { g/g sludge } \\
\text { (oxide) }\end{array}$ & $\begin{array}{c}\% \text { element } \\
\text { dissolved }\end{array}$ & $\begin{array}{r}\text { ug/g sludge } \\
\text { (element) }\end{array}$ & $\underset{\text { (element) }}{\mathbf{k g} / \text { tank ug }}$ & $\begin{array}{l}\text { g sludge } \\
\text { (oxide) }\end{array}$ & $\begin{array}{l}\text { \% element } \\
\text { dissolved }\end{array}$ & $\begin{array}{l}\text { ug/g sludge } \\
\text { (element) }\end{array}$ & $\begin{array}{r}\mathrm{kg} / \operatorname{tank} \\
\text { (element) }\end{array}$ \\
\hline Áluminum & 1.889 & 3.010 & 12 & 0.39 & 775.000 & 319.2 & 1463.975 & 99.61 & 0.000 & 0.0 & 0.000 & 0.00 & 778.010 & 320.4 \\
\hline Barium & 1.117 & 6.070 & 2.5 & 49.35 & 6.230 & 2.6 & 6.959 & 50.65 & 0.000 & 0.0 & 0.000 & 0.00 & 12.300 & 5.1 \\
\hline Bismuth & 1.115 & 9.730 & 4.0 & 17.98 & 44.400 & 18.3 & 49.506 & 82.02 & 0.000 & 0.0 & 0.000 & 0.00 & 54130 & 22.3 \\
\hline Boron & 3.220 & NA & 0.0 & HDivio! & 0.000 & 0.0 & 0.000 & \#DIVIo! & 0.000 & 0.0 & 0.000 & HDIVIo! & 0.000 & 0.0 \\
\hline Cadmium & 1.142 & 2.210 & 0.8 & 46.72 & 2.520 & 1.0 & 2.878 & 53.28 & 0.000 & 0.0 & 0.000 & 0.00 & 4.730 & 1.9 \\
\hline Calcium & 1.399 & NA & 0.0 & HDIVIO! & 0.000 & 0.0 & 0.000 & $\# D \mid V / O !$ & 0.000 & 0.0 & 0.000 & HDIV/o! & 0.000 & 0.0 \\
\hline Chromium & 1.823 & 8.050 & 3.3 & 7.78 & 95.400 & 39.3 & 183.454 & 92.22 & 0.000 & 0.0 & 0.000 & 0.00 & 103.450 & 426 \\
\hline Cobalt & 1.271 & NA & 0.0 & HDIVIO! & 0.000 & 0.0 & 0.000 & HOIV/O! & 0.000 & 0.0 & 0.000 & \#DIVIo' & 0.000 & 0.0 \\
\hline Copper & 1.252 & NA & 0.0 & ADIV/O! & 0.000 & 0.0 & 0.000 & $\# \mathrm{D} \mid \mathrm{Y} / \mathrm{OI}$ & 0.000 & 0.0 & 0.000 & \#DIV/o! & 0.000 & 00 \\
\hline Iron & 1.382 & 1.150 & 0.5 & 0.09 & 1210.000 & 498.3 & 1672.220 & 99.91 & 0.000 & 0.0 & 0.000 & 0.00 & 1211.150 & 498.8 \\
\hline Lead & $\$ .077$ & 7.170 & 3.0 & 10.52 & 61.000 & 25.1 & 65.697 & 89.48 & 0.000 & 0.0 & 0.000 & 0.00 & 68.170 & 28.1 \\
\hline Magnesium & 1.658 & NA & 0.0 & \#DIV/O! & 0.000 & 0.0 & 0.000 & \#DIVIO! & 0.000 & 0.0 & 0.000 & \#DIV/O! & 0.000 & 0.0 \\
\hline Manganese & 1.582 & 1.770 & 0.7 & 5.85 & 28.000 & 11.5 & 44.296 & 94.05 & 0.000 & 0.0 & 0.000 & 0.00 & 29.770 & 12.3 \\
\hline Nickel & 1.272 & 1.420 & 0.6 & 6.92 & 19.100 & 7.9 & 24.295 & 93.08 & 0.000 & 0.0 & 0000 & 0.00 & 20.520 & 8.5 \\
\hline Prospriate & 1.000 & 25200.000 & 10377.6 & 89.81 & 2860.000 & 1177.8 & 2860.000 & 10.19 & 0.000 & 0.0 & 0.000 & 0.00 & 28060.000 & 11555.3 \\
\hline Potassium & 1.000 & NA & 0.0 & \#DIV/0! & 0.000 & 0.0 & 0.000 & \#DIV/o! & 0.000 & 0.0 & 0.000 & \#DIV/o! & 0.000 & 0.0 \\
\hline Silicon & 2.139 & 78.700 & 32.4 & 21.06 & 295.000 & 121.5 & 631.005 & 78.94 & 0.000 & 0.0 & 0.000 & 0.00 & 373.700 & 153.9 \\
\hline Silver & 1.000 & 0.885 & 0.4 & 47.40 & 0.982 & 0.4 & 0.982 & 52.60 & 0.000 & 0.0 & 0.000 & 0.00 & 1.867 & 0.8 \\
\hline Sodium & 1.000 & 1980000.000 & 81538.0 & 96.28 & 7660.000 & 3154.4 & 7660.000 & 3.72 & 0.000 & 0.0 & 0.000 & 0.00 & 205660.000 & 84692.4 \\
\hline Strontium & 1.183 & NA & 0.0 & HDIV/OI & 0.000 & 0.0 & 0.000 & \#DIVIo! & 0.000 & 0.0 & 0.000 & \#DIV/O! & 0.000 & 0.0 \\
\hline Zinc & 1.245 & NA & 0.0 & \#DIVIo! & 0.000 & 0.0 & 0.000 & ADIVIO! & 0.000 & 0.0 & 0.000 & \#DIV/0! & 0.000 & 0.0 \\
\hline Zirconium & 1.351 & 4.070 & 1.7 & 42.53 & 5.500 & 23 & 7.431 & 57.47 & 0.000 & 0.0 & 0.000 & 0.00 & 9.570 & 3.9 \\
\hline Uranium & 1.134 & 8.850 & 3.6 & 4.61 & 183.000 & 75.4 & 207.522 & 95.39 & 0.000 & 0.0 & 0.000 & 0.00 & 191.850 & 79.0 \\
\hline Nitrate & 1.000 & 444000.000 & 182842.8 & 100.00 & NA & 0.0 & 0.000 & 0.00 & 0.000 & 0.0 & 0.000 & 0.00 & 444000.000 & 182842.8 \\
\hline Chloride & 1.000 & NR. & 0.0 & ADIVIOI & NA & 0.0 & 0.000 & \#DIV/OI & 0.000 & 0.0 & 0.000 & \#DIV/0! & 0.000 & 0.0 \\
\hline Fluoride & 1.000 & NR & 0.0 & \#DIV/0! & NA & 0.0 & 0.000 & ADIV/OI & 0.000 & 0.0 & 0.000 & HDIV/O! & 0.000 & 0.0 \\
\hline Free $\mathrm{OH}$ & 1.000 & 5700.000 & 2347.3 & 100.00 & NA & 0.0 & 0.000 & 0.00 & 0.000 & 0.0 & 0.000 & 0.00 & 5700.000 & 2347.3 \\
\hline Carbonate & 1.000 & NR & 0.0 & \#DIV/0! & NA & 0.0 & 0.000 & \#DIV/O! & 0.000 & 0.0 & 0.000 & \#DIVIO! & 0.000 & 0.0 \\
\hline TOC & 1.336 & 227.000 & 70.0 & 100.00 & NA & 0.0 & 0.000 & 0.00 & 0.000 & 0.0 & 0.000 & 0.00 & 169.910 & 70.0 \\
\hline Nitrite & 1.000 & 1000.000 & 411.8 & 100.00 & NA & 0.0 & 0.000 & 0.00 & 0.000 & 0.0 & 0.000 & 0.00 & 1000.000 & 411.8 \\
\hline Sulfate & 1.000 & 14000.000 & 5765.3 & 100.00 & MA & 0.0 & 0.000 & 0.00 & 0.000 & 0.0 & 0.000 & 0.00 & 14000.000 & 5765.3 \\
\hline \multicolumn{2}{|l|}{ Total } & $\begin{array}{r}688260.085 \\
96.94\end{array}$ & \multicolumn{2}{|l|}{$\begin{array}{l}\text { \%total solids } \\
\text { in wash }\end{array}$} & \multicolumn{4}{|c|}{$\begin{array}{r}14880.220 \\
2.10\end{array}$} & \multicolumn{4}{|c|}{0.000} & \multicolumn{2}{|c|}{$\begin{array}{l}99.03 \% \text { mass } \\
\text { balance }\end{array}$} \\
\hline \multicolumn{2}{|l|}{ Radionuclides } & $\mathrm{uCl} / \mathrm{g}$ & Ci/tank & dissolved & $\mathrm{uCi} / \mathrm{g}$ & Ci/tank & & dissolved & $\mathrm{uCi} / \mathrm{g}$ & Ci/tank & & dissolved & $\mathrm{uCi} / \mathrm{g}$ & Cítank \\
\hline \multicolumn{2}{|l|}{$P u-239,240$} & 0.001 & 0.4 & 100.00 & & 0.0 & & 0.00 & 0.000 & 0.0 & & $0.0 \overline{0}$ & 0.001 & 0.4 \\
\hline \multicolumn{2}{|l|}{$C-14$} & 0.000 & 0.0 & HDIVIO! & & 0.0 & & \#DIV/O! & 0.000 & 0.0 & & HDIV/O! & 0.000 & 0.0 \\
\hline \multicolumn{2}{|l|}{ St-90 } & 0.056 & 23.1 & 1.23 & 4.510 & 1857.3 & & 98.77 & 0.000 & 0.0 & & 0.00 & 4.566 & 1880.3 \\
\hline \multicolumn{2}{|l|}{ TC- 99} & 0.000 & 0.0 & \#DIV/o! & & 0.0 & & \#DIV/O! & 0.000 & 0.0 & & \#DIVIo! & 0.000 & 0.0 \\
\hline \multicolumn{2}{|l|}{ Am-241 } & 0.002 & 0.8 & 40.00 & 0.003 & 1.2 & - & 50.00 & 0.000 & 0.0 & & 0.00 & 0.005 & 2.1 \\
\hline \multicolumn{2}{|l|}{$\mathrm{Co}-60$} & 0.001 & 0.4 & 50.00 & 0.001 & 0.4 & & 50.00 & 0.000 & 0.0 & & 0.00 & 0.002 & 0.8 \\
\hline \multirow{2}{*}{\multicolumn{2}{|c|}{ Cs -137}} & 2.620 & 1078.9 & 78.75 & 0.707 & 291.1 & & 21.25 & 0.000 & 0.0 & & 0.00 & 3.327 & 1370.1 \\
\hline & & 0.000 & 0.0 & \#DIVIOI & & 0.0 & & \#DIV/0! & 0.000 & 0.0 & & \#DIV/OI & 0.000 & 0.0 \\
\hline
\end{tabular}

NOTES 
TX-116 (EB, 1C BiPO4)

$\begin{array}{lr}\text { Density }(\mathrm{g} / \mathrm{mL}) & 1.7 \\ \text { Volume } & (\mathrm{L})\end{array}$

$\begin{array}{ll}\text { Volume (L) } & 2388335 \\ \text { Mass }(\mathrm{kg}) & 4060170\end{array}$

Mass $(\mathrm{kg})$
Solids $(\mathrm{ug} / \mathrm{g}) \quad 645000$

$\sim \mathrm{pH} \quad 13.0$

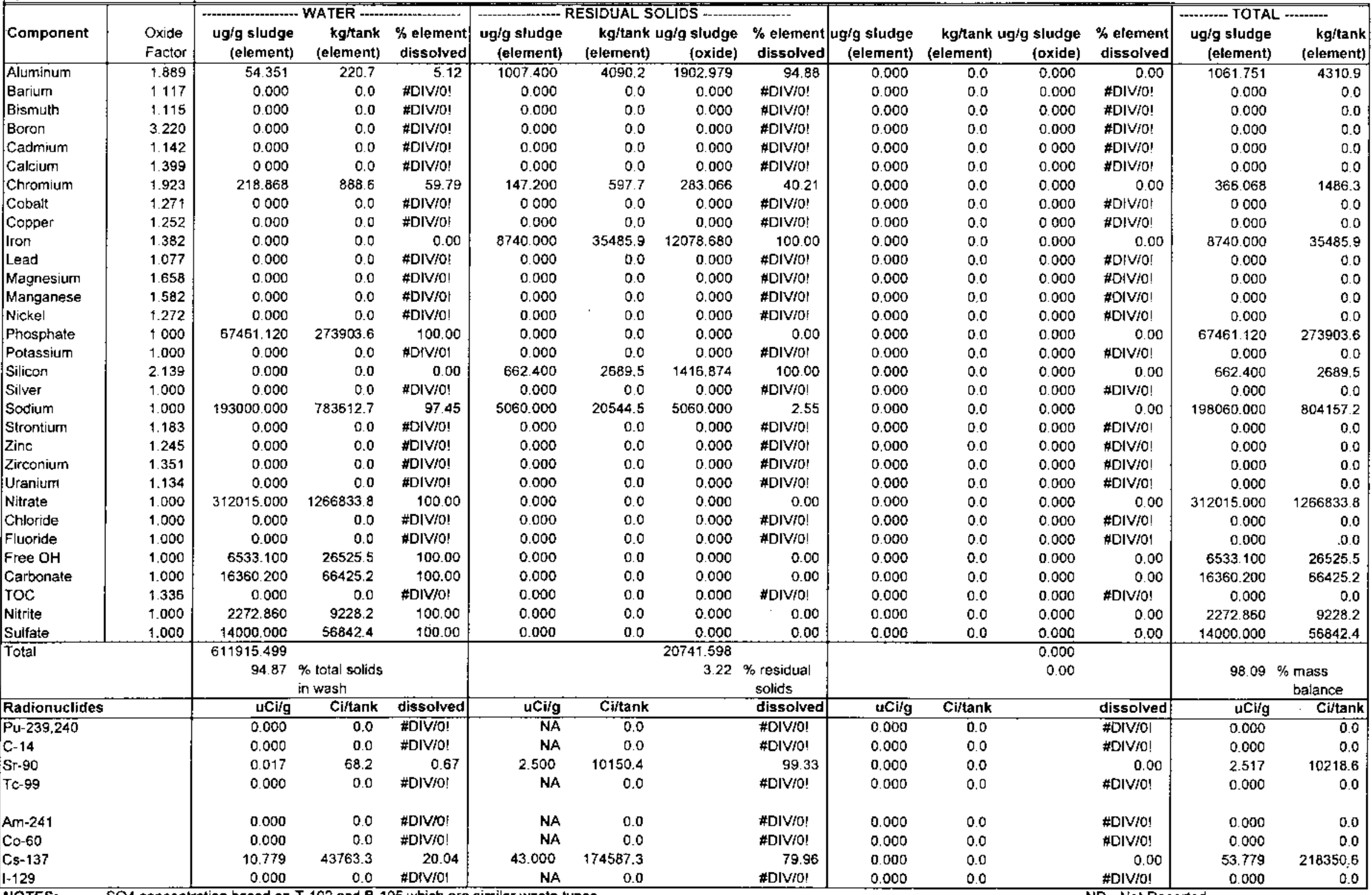
$\mathrm{SO} 4$ concentration based on $\mathrm{T}-102$ and $\mathrm{B}-105$ which are similar waste types

作

NR - Not Reported 0.000

$\mathrm{Na}$ concentration adjusted to ion belance.

NA - No Analysis Performed

Percent solids based on average of TY-102 (71\% solids, PO4=25,200 ug/g) and B-105 (58\%, PO4=104,000 ug/g): TX-116 (65\% solids, PO4=67,500 ug/g) 


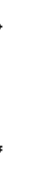




\section{Appendix B}

\section{Experimental Washing and Leaching Data}

Summary Worksheets 
B-104 (BjPO4 2C, EB) Experimental Results

\begin{tabular}{|c|c|c|c|c|c|c|c|c|c|}
\hline & $\begin{array}{l}\text { Density }\left(g^{\prime / m L}\right) \\
\text { Volume }(k L)\end{array}$ & & & & & & & & \\
\hline & summation & & & & Wa & & & Leach & \\
\hline Component & $\begin{array}{l}\text { ugig sample } \\
\text { [Temer 1996] }\end{array}$ & $\begin{array}{r}\% \\
\text { recovery }\end{array}$ & $\begin{array}{l}\text { ug/g sample } \\
\text { (Appendix) }\end{array}$ & $\begin{array}{r}M T \\
\text { (tank) }\end{array}$ & $\begin{array}{l}\text { \% removed } \\
\text { initial solids }\end{array}$ & MT removed & $\begin{array}{r}\% \text { removed } \\
\text { washts solids }\end{array}$ & MT removed & $\begin{array}{r}\text { \% leach } \\
\text { Inltial solids }\end{array}$ \\
\hline $\mathrm{Ag}$ & & & & 0.00 & & 0.000 & 0 & 0.000 & \\
\hline $\mathrm{Al}$ & 1310 & 90 & 1840 & 3.36 & 6 & 0.202 & 60 & 1.901 & 57 \\
\hline As & & & & 000 & & 0.000 & 0 & 0.000 & \\
\hline$\theta$ & 75.3 & 111 & & 0.00 & 64 & 0.000 & 21 & 0000 & 8 \\
\hline $\mathrm{Ba}$ & 7.37 & 106 & & 000 & 0 & 0.000 & 0 & 0.000 & D \\
\hline $\mathrm{Be}$ & & & & 0.00 & & 0.000 & 0 & 0000 & \\
\hline $\mathrm{Bi}$ & 12000 & 98 & 11400 & 20.81 & 0 & 0.000 & 3 & 0708 & 3 \\
\hline $\mathrm{Ca}$ & 217 & 105 & 328 & 0.60 & 0 & 0.000 & 0 & 0.000 & 0 \\
\hline $\mathrm{Cu}$ & & & & 000 & 33 & 0.000 & 0 & 0.000 & 0 \\
\hline $\mathrm{Ce}$ & & & & 000 & & 0.000 & 0 & 0.000 & \\
\hline Co & & & & 0.00 & & 0000 & a & 0.000 & \\
\hline $\mathrm{Cr}$ & 526.29 & & 513 & 0.94 & 3 & 0028 & 71 & 0.644 & 69 \\
\hline $\mathrm{Cu}$ & 526 & & & 0.00 & & 0.000 & 0 & 0.000 & \\
\hline $\mathrm{Fe}$ & 12000 & 104 & 10800 & 19.72 & 0 & 0.000 & 1 & 0.099 & 1 \\
\hline $\mathrm{k}$ & 490 & 119 & & 0.89 & 48 & 0.429 & 90 & 0.420 & 47 \\
\hline La & 0.63 & 81 & & 0.00 & & 0.000 & 0 & 0.000 & 0 \\
\hline $\mathrm{Li}$ & & & & 0,00 & & 0.000 & 0 & 0.000 & \\
\hline $\mathrm{Mg}$ & 136 & 99 & & ‥ 25 & 0 & 0.000 & 8 & 0.019 & 8 \\
\hline Mn & 359 & 107 & & 0.07 & 0 & 0.000 & 0 & 0.000 & 0 \\
\hline Mo & 178 & 105 & & 0.00 & 96 & 0.003 & 65 & 0.000 & 3 \\
\hline $\mathrm{Na}$ & (1.30000 direct $\}$ & & 117000 & 213.58 & 93 & 198.633 & 0 & 0.000 & \\
\hline $\mathrm{N}_{1}$ & 169 & 70 & & 0.03 & 2 & 0.001 & 0 & 0.000 & \\
\hline $\mathrm{Pb}$ & 102 & 98 & & 0.19 & 0 & 0.000 & 17 & 0.031 & 17 \\
\hline $\mathrm{Pd}$ & 571 & 604 & & 0.01 & 4 & 0,000 & 11 & 0.001 & 11 \\
\hline $\mathrm{Rh}$ & & & & 0.00 & & 0.000 & 0 & 0.000 & \\
\hline$R u$ & & & & 0.00 & & 0.000 & 0 & 0.000 & \\
\hline $\mathrm{Sb}$ & & & & 0.00 & & 0.000 & 0 & 0.000 & \\
\hline $\mathrm{Se}$ & & & & 0.00 & & 0.000 & 0 & 0.000 & \\
\hline $\mathrm{Si}$ & 3020 & 677 & 5700 & 10.41 & 1 & 0.104 & 50 & 5.140 & 49 \\
\hline$S_{n}$ & & & & 0.00 & & 0,000 & 0 & 0.000 & \\
\hline $\mathrm{Sr}$ & 150 & 99 & & 0.27 & 0 & 0.000 & 0 & 0.000 & 0 \\
\hline Te & & & & 0,0 & & 0.000 & 0 & 0.000 & \\
\hline Th & & & & 000 & & 0.000 & 0 & 0,000 & \\
\hline$T_{1}$ & & & & 0.00 & & 0000 & 0 & 0.000 & \\
\hline U & 1380 & 94 & 1100 & 2.01 & 1 & 0.020 & 36 & 0.707 & 35 \\
\hline$v$ & & & & 000 & & 0.000 & 0 & 0.000 & \\
\hline$w$ & & & & 0.00 & & 0.000 & 0 & 0.000 & \\
\hline$y$ & & & & 0.00 & & 0000 & 0 & 0,000 & \\
\hline$Z n$ & 30.3 & 50.4 & & 000 & 5 & 0.000 & 57 & 0.000 & 55 \\
\hline $\mathrm{Zr}$ & 24.8 & 90 & & 0.05 & 1 & 0.0005 & 1 & 0.001 & 1 \\
\hline
\end{tabular}

\begin{tabular}{|c|c|c|c|c|c|c|c|c|c|}
\hline \multirow[b]{2}{*}{ Component } & \multirow{2}{*}{$\begin{array}{l}\text { summation } \\
\text { ugig sample } \\
\text { (Temer 1996) }\end{array}$} & \multirow[b]{2}{*}{$\begin{array}{r}\% \\
\text { recovery }\end{array}$} & \multirow[b]{2}{*}{$\begin{array}{r}\text { uglg sample } \\
\text { (Appendix) }\end{array}$} & \multirow[b]{2}{*}{$\begin{array}{r}\mathbf{H T} \\
{[\operatorname{tank}]}\end{array}$} & \multicolumn{2}{|c|}{ Wash } & \multicolumn{3}{|c|}{ Leach } \\
\hline & & & & & $\begin{array}{l}\text { \% removed } \\
\text { initial solids }\end{array}$ & MT removed & $\begin{array}{r}\% \text { remaved } \\
\text { washed solids }\end{array}$ & MT kemoved & $\begin{array}{l}\% \text { removed } \\
\text { initial solids }\end{array}$ \\
\hline $\mathrm{OH}-$ & & & & 0.00 & & 0.000 & 0 & 0.000 & \\
\hline NO3- & & & 290000 & 529.40 & & 0.000 & 0 & 0.000 & \\
\hline $\mathrm{NO}_{2}$. & & & 2450 & 4.47 & & 0.000 & 0 & 0.000 & \\
\hline $\mathrm{PO} 4.3$ & 57600 & 105 & 53400 & 97.48 & 45 & 43.867 & 98 & 52543 & 54 \\
\hline SO4-2 & & & 21400 & 39.07 & & 0.000 & 0 & 0.000 & \\
\hline F- & & & 3460 & 6.32 & & 0.000 & 0 & 0000 & \\
\hline C. & & & 2110 & 3.85 & & - 0.000 & 0 & 0.000 & \\
\hline \multirow[t]{2}{*}{ Br- } & & & & 0.00 & & 0.000 & 0 & 0.000 & \\
\hline & $\begin{array}{r}\text { summation } \\
\text { ucilg sample } \\
\text { (Temer 1996) }\end{array}$ & $\begin{array}{r}\% \\
\text { recovery }\end{array}$ & $\begin{array}{l}\text { uCi/g sample } \\
\text { (Appendix) }\end{array}$ & $\begin{array}{r}\mathrm{Ci} \\
{[\tan k]}\end{array}$ & $\begin{array}{l}\text { \% removed } \\
\text { initial solids }\end{array}$ & Ci removed & $\begin{array}{l}\%_{0} \text { removed } \\
\text { washed solids }\end{array}$ & Ci removed & $\begin{array}{l}\% \text { removod } \\
\text { initial solids }\end{array}$ \\
\hline Total alpha & 0.0859 & 35 & 0.04 & 73.02 & & 0.000 & 0 & 0.000 & \\
\hline Pu-239, 240 & . 00647 & 84 & & 118.11 & & 0.000 & $<\mathrm{DL}$ & AVALUEI & \\
\hline$A m-241+P \cup-238$ & 0.0005 & 37 & & 1.64 & & 0.000 & $<\mathrm{DH}$ & WVALUE! & \\
\hline Am-241 (gamma) & & & & 000 & & 0.040 & 0 & 0.000 & \\
\hline $\mathrm{Cm}-243,24 \mathrm{~A}$ & & & & 0.00 & & 0000 & 0 & 0.000 & \\
\hline Cs-137 & 5.13 & 103 & & 9364.84 & & 0.000 & 99 & 9299.289 & \\
\hline Co-60 & & & & 0.00 & & 0000 & 0 & 0.000 & \\
\hline Eu-154 & & & & 000 & & 0000 & 0 & 0.000 & \\
\hline Eu-155 & & & & 000 & & 0000 & 0 & 0.000 & \\
\hline Sr.90 & 10.3 & & & 1880271 & & 0.000 & 0 & 0.000 & \\
\hline Tc-99 & $<0.02$ & & & 0.00 & & 0.000 & 0 & 0.000 & \\
\hline
\end{tabular}

Notes:

Characterizaticn data worksheet in Appendix

Temer. D. J. and R Vallarreat 1996. Siudge Washing and Aikaline Legching Tests on Actual Hantord Tank Situdge: FY 1996 Results, LA!JR96-2839, Los Alamos National Laboratory. Los Alamos, New Mexico.

B. 1 
B.110 (BiPO4 2C, 5-6) Experimental Results

\begin{tabular}{|c|c|c|c|c|c|c|c|c|c|}
\hline \multicolumn{10}{|c|}{\begin{tabular}{|c|c|}
1.35 Density $(\mathrm{g} / \mathrm{mL})$ \\
927 Volume $(\mathrm{kL})$ \\
\end{tabular}} \\
\hline \multirow[b]{2}{*}{ Component } & \multirow{2}{*}{$\begin{array}{r}\text { range } \\
\text { ugtg sample } \\
\text { (Lumetta 1994) }\end{array}$} & \multirow[b]{2}{*}{$\begin{array}{r}\% \\
\text { recovery } \\
\end{array}$} & \multirow[b]{2}{*}{$\begin{array}{r}\text { ug'g sample } \\
\text { (Cotton 1995) }\end{array}$} & \multirow[b]{2}{*}{$\begin{array}{r}\text { MT } \\
(\operatorname{tank})\end{array}$} & \multicolumn{2}{|c|}{ Wash } & \multicolumn{3}{|c|}{ Leach } \\
\hline & & & & & $\begin{array}{l}\text { \% temoved } \\
\text { initial solids }\end{array}$ & MT removid & $\begin{array}{r}\% \text { removed } \\
\text { wasthed solids }\end{array}$ & MT removed & $\begin{array}{r}\% \text { leach } \\
\text { initlal solids }\end{array}$ \\
\hline $\mathrm{Ag}$ & & & 86.7 & 0.11 & & 0.000 & 0 & 0.000 & \\
\hline Al & $700-2200$ & & 1230 & 1.54 & 0 & 0.000 & 0 & 0.000 & 0 \\
\hline$A_{5}$ & & & & 0.00 & & 0.000 & 0 & 0.000 & \\
\hline B & & & 34.5 & 0,04 & & 0.000 & 0 & 0.000 & \\
\hline $\mathrm{Ba}$ & & & 24.6 & 0.03 & & 0.000 & 0 & 0.000 & \\
\hline Be & & & & 0.00 & & 0,000 & 0 & 0.000 & \\
\hline $\mathrm{Bi}$ & $21000 \cdot 25000$ & & 20100 & 25.16 & 0 & 0.000 & 0 & 0.000 & 0 \\
\hline $\mathrm{Ca}$ & $870-1800$ & & 967 & 1.21 & 10 & 0.121 & 10 & 0.109 & 9 \\
\hline cod & & & 30 & 0.04 & & 0.000 & 0 & 0.000 & \\
\hline Ce & & & & 0.00 & & 0.000 & 0 & 0.000 & \\
\hline $\mathrm{Co}$ & & & & 0.50 & & 0.000 & 0 & 0.000 & \\
\hline $\mathrm{Cr}$ & $890-970$ & & 972 & 1.22 & 10 & 0.122 & 53 & 0.584 & 48 \\
\hline $\mathrm{Cu}$ & & & 59.3 & 0.07 & 0 & 0.000 & 0 & 0.000 & 0 \\
\hline $\mathrm{Fe}$ & $20000-23000$ & & 19300 & 24.16 & 0 & 0000 & 0 & 0.000 & \\
\hline$K$ & & & 467 & 0.58 & & 0.000 & 0 & 0.000 & \\
\hline La & & & & 000 & & 0.000 & to & 0.000 & \\
\hline it & & & & $O D O$ & & 0.000 & 0 & 0.000 & \\
\hline $\mathrm{Mg}$ & $150-180$ & & 191 & 024 & 9 & 0022 & 0 & 0.000 & 0 \\
\hline Mn & $72 \cdot 130$ & & 90.3 & 0.11 & 0 & 0.000 & 0 & 0.000 & 0 \\
\hline$M 0$ & & & & 000 & & 0.000 & 0 & 0.000 & \\
\hline $\mathrm{Na}$ & $99000-140000$ & & 100000 & 125.19 & 93 & 116.426 & 0 & 0.000 & \\
\hline $\mathrm{Ni}$ & & & 885 & 0.11 & & 0.000 & 0 & 0.000 & \\
\hline $\mathrm{Pb}$ & $960 \cdot 1300$ & & 657 & 0.82 & 0 & 0.000 & 0 & 0,000 & 0 \\
\hline Pd & & & & 0.00 & & 0.000 & 0 & 0000 & \\
\hline Rh & & & & 0.00 & & 0.000 & 0 & 0.000 & \\
\hline Ru & & & & 0.00 & & 0.000 & 0 & 0.000 & \\
\hline So & & & & 0.00 & & 0.000 & 0 & 0.000 & \\
\hline Se & & & & 0.00 & & 0.000 & 0 & 0.000 & \\
\hline Si & $7000-14000$ & & 9850 & 12.33 & 28 & 3.453 & 53 & 4,686 & 38 \\
\hline Sn & & & & 0.00 & & 0.000 & 0 & 0.000 & \\
\hline $\mathrm{Sr}$ & $220 \cdot 270$ & & 217 & 0.27 & 0 & 0.000 & 0 & 0.000 & 0 \\
\hline Té & & & & 0.00 & & 0.000 & 0 & 0.000 & \\
\hline Th & & & & 0.00 & & 0.000 & 0 & 0.000 & \\
\hline$T_{1}$ & & & & 0.00 & & 0.000 & 口 & 0.000 & \\
\hline u & $610 \cdot 1900$ & & 404 & 0.51 & 14 & 0.071 & 34 & 0.147 & 29 \\
\hline V & & & & 0.00 & & 0.000 & 0 & 0.000 & \\
\hline$w$ & & & & 0.00 & & 0.000 & 0 & 0.000 & \\
\hline$y$ & & & & 0.00 & & 0.000 & 0 & 0.000 & \\
\hline $\mathrm{Zn}$ & $64 \cdot 180$ & & 503 & 0.63 & 0 & 0.000 & 0 & 0.000 & 0 \\
\hline$Z r$ & & & 3.5 & 0.00 & & 0.000 & 0 & 0.000 & \\
\hline
\end{tabular}

\begin{tabular}{|c|c|c|c|c|c|c|c|c|c|}
\hline \multirow[b]{2}{*}{ Companent } & \multirow{2}{*}{$\begin{array}{r}\text { range } \\
\text { uglg sample } \\
\text { (Lumetta 1994) }\end{array}$} & \multirow[b]{2}{*}{$\begin{array}{r}\% \\
\text { recovery }\end{array}$} & \multirow[b]{2}{*}{$\begin{array}{l}\text { uglg satmple } \\
\text { (Colton 1995) }\end{array}$} & \multirow[b]{2}{*}{$\begin{array}{r}M T \\
\text { ttank }\end{array}$} & \multicolumn{2}{|c|}{ Wash } & \multicolumn{3}{|c|}{ Leach } \\
\hline & & & & & $\begin{array}{l}\text { \% removed } \\
\text { initial solids }\end{array}$ & MT removed & $\begin{array}{l}\% \text { removed } \\
\text { washed sollds }\end{array}$ & MT removed & $\begin{array}{l}\% \text { removed } \\
\text { initial solids }\end{array}$ \\
\hline $\mathrm{OH}-$ & & & & 0.00 & & 0.000 & 穴 & 0.000 & \\
\hline $\mathrm{NO}_{3}$ & & & 181000 & 226.59 & & 0000 & 0 & 0.000 & \\
\hline $\mathrm{NO} 2$ & & & 10400 & 13.02 & & 0.000 & 0 & 0.000 & \\
\hline PD4-3 & $54000-60000$ & & 52300 & 65.47 & 84 & 54.998 & 88 & 9.166 & 14 \\
\hline SO $4-2$ & & & 11300 & 14.15 & & 0.000 & 0 & 0.000 & \\
\hline F- & & & 1780 & 2.23 & & 0.000 & D & 0.000 & \\
\hline C]. & & & 1150 & 1.44 & & 0.000 & 0 & 0.000 & \\
\hline \multirow[t]{2}{*}{ Вг- } & & & & 0.00 & & 0.000 & 0 & 0.000 & \\
\hline & $\begin{array}{r}\text { range } \\
\text { uCitg sample } \\
\text { [Lumetta 1994] }\end{array}$ & $\begin{array}{r}\% \\
\text { recovery }\end{array}$ & $\begin{array}{l}\text { uCiig sample } \\
\text { [Colton t995) }\end{array}$ & $\begin{array}{r}\mathrm{ci} \\
\text { tank\} }\end{array}$ & $\begin{array}{l}\% \text { removed } \\
\text { initial sallds }\end{array}$ & Ci removed & $\begin{array}{l}\% \text { removed } \\
\text { washed solids }\end{array}$ & Ci removed & $\begin{array}{l}\text { \% removed } \\
\text { initial solids }\end{array}$ \\
\hline Total alpha & & & & 000 & & 0.000 & 0 & 0000 & \\
\hline Pu-239, 24C & & & 0.116 & 145.22 & & 0.000 & 0 & 0.000 & \\
\hline$A_{\pi T}=241+P u-238$ & & & & 0.00 & & 0.000 & 0 & 0.000 & \\
\hline Arri-241 (gamma) & & & 0.082 & 102.65 & & 0,000 & 0 & 0.000 & \\
\hline $\mathrm{Cm} \cdot 243,244$ & & & & 0.00 & & 0.000 & 0 & 0.000 & \\
\hline $\operatorname{cs}-137$ & $146 \cdot 160$ & & 15.1 & 18903.52 & 87 & 16446.063 & 62 & 1512.282 & 8 \\
\hline Co.60 & & & & 0.00 & & 0.000 & 0 & 0.000 & \\
\hline Eu-154 & & & & 0.00 & & 0.000 & 0 & 0.000 & \\
\hline Eu-155 & & & & 0.00 & & 0.000 & 0 & 0.000 & \\
\hline Sr-90 & $169-250$ & & 169 & 211569.20 & 0 & 0.000 & 0 & 211.569 & 0.1 \\
\hline Te-99 & 0.026 & & 0.02 & 25.04 & 94 & 23.536 & 100 & 1.502 & 6 \\
\hline
\end{tabular}

Notes:

Less than wash, leach values enterett above as zero

Colton N O 1995. Siludge Pretreatment Chemistry Evaluation: Enhanced Studge Washing Separation Factors, PNL-10512

Pacific Northwest Natıonal Laboratory. Rıchland washington

Lumetta, G. J. and $8 \mathrm{M}$ Rapko. 1994. Washing and Alkaline Leaching of Hanford Studges: A Stafus Report

PNL-10078, Pacific Northweșt National Laboratory Richtand, Washington.

B. 2 
8-111 (BiPO4 2C, 5-6) Experimental Results

\begin{tabular}{|c|c|c|c|c|c|c|c|c|c|}
\hline 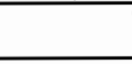 & $\begin{array}{l}\text { Density }(\mathrm{g} / \mathrm{mL}) \\
\text { Volume }(\mathrm{kL})\end{array}$ & & & & & & & & \\
\hline & summation & & & & $\mathrm{Wa}$ & & & Leach & \\
\hline Component & $\begin{array}{l}\text { ugio sample } \\
\text { (Rapko 1995) }\end{array}$ & $\begin{array}{r}\% \\
\text { recovery }\end{array}$ & $\begin{array}{r}\text { ugig sample } \\
\text { (Colton 1995) }\end{array}$ & $\begin{array}{r}M T \\
\{\operatorname{tank}\}\end{array}$ & $\begin{array}{l}\% \text { removed } \\
\text { initial solids }\end{array}$ & MT removed & $\begin{array}{l}\% \text { removed } \\
\text { washed solids }\end{array}$ & MT removed & $\begin{array}{l}\% \text { leach } \\
\text { initial solids }\end{array}$ \\
\hline$\overline{A g}$ & 40.3 & & 97.4 & 0.11 & 0 & 0.000 & 0.0 & 0.000 & 0 \\
\hline $\mathrm{AF}$ & 1480 & 137 & 1360 & 1.52 & $B$ & 0,000 & 20 & 0.030 & 2 \\
\hline$A_{5}$ & & & & 0.00 & & 0.000 & 0.0 & 0.000 & \\
\hline$B$ & 105 & & 68.4 & 0.08 & 11 & 0.008 & 4.5 & 0.003 & 4 \\
\hline $\mathrm{Ba}$ & 23.4 & 185 & 42.3 & 005 & 0 & 0.000 & 0.0 & 0,000 & 0 \\
\hline $\mathrm{Be}$ & & & & 0.00 & & 0.000 & 0.0 & 0.000 & \\
\hline $\mathrm{Bi}$ & 14600 & 85 & 20200 & 22.55 & 0 & 0.000 & 0.0 & 0.000 & 0 \\
\hline $\mathrm{Ca}$ & 592 & 149 & 683 & 076 & 9 & 0.069 & 2.2 & 0.015 & 2 \\
\hline $\mathrm{Cd}$ & 2.42 & & 200 & 002 & 0 & 0.000 & 0.0 & 0000 & 0 \\
\hline $\mathrm{Ce}$ & 14.3 & & & 002 & 0 & 0,000 & 0.0 & 0.000 & 0 \\
\hline $\mathrm{Co}$ & 3.33 & & 16 & 002 & 0 & 0000 & 0.0 & 0.000 & 0 \\
\hline $\mathrm{Cr}$ & 950 & & 1150 & 1.28 & 27 & 0.347 & 19.2 & 0.180 & 14 \\
\hline $\mathrm{Cu}$ & 191 & & 221 & 0.25 & 1 & 0.002 & 12.1 & 0.030 & 12 \\
\hline $\mathrm{Fe}$ & 14500 & 88 & 16400 & 18.31 & 0 & 0.000 & 0.0 & 0.000 & 0 \\
\hline$K$ & & & 613 & 0.68 & & 0.000 & 0.0 & 0.000 & \\
\hline La & 429 & & & 0.00 & 0 & 0.000 & 0.0 & 0.000 & 0 \\
\hline 23 & & & & 0.00 & & 0.000 & 0.0 & 0.000 & \\
\hline $\mathrm{Mg}$ & & & 738 & 0.82 & 0 & 0,000 & 0.0 & 0.000 & 0 \\
\hline $\mathrm{Mn}$ & 194 & 55 & 111 & 0.12 & $D$ & 0.000 & 0.0 & 0.000 & 0 \\
\hline Mo & 382 & & & 0.00 & 87 & 0.000 & 30.8 & 0.000 & 4 \\
\hline $\mathrm{Na}$ & & & 87400 & 97.59 & 92 & 89.782 & 0.0 & 0.000 & \\
\hline $\mathrm{Ni}$ & & & 18.6 & 0.02 & & 0.000 & 0.0 & 0.000 & \\
\hline $\mathrm{Pb}$ & 1200 & 83 & & 1.34 & 0 & 0.000 & 5.0 & 0.067 & 5 \\
\hline $\mathrm{Pd}$ & & & & 0.00 & & 0.000 & 0.0 & 0.000 & \\
\hline $\mathrm{Rh}$ & & & & 0,00 & & 0.000 & 0.0 & 0.000 & \\
\hline $\mathrm{Ru}$ & & & & 0.00 & & 0,000 & 0.0 & 0.000 & \\
\hline Se & 24.2 & & & 0.03 & 0 & 0.000 & 0.0 & 0.000 & 0 \\
\hline$S_{1}$ & 9900 & 106 & 9640 & 10.76 & 3 & 0.323 & 46.4 & 4.844 & 45 \\
\hline Sn & & & & 0.00 & & 0.000 & 0.0 & 0.000 & \\
\hline $\mathrm{Sr}$ & 170 & 81 & 221 & 0.25 & 0 & 0.000 & 0.0 & 0.000 & 0 \\
\hline$T_{e}$ & & & & 0.00 & & 0.000 & 0.0 & 0.000 & \\
\hline Th & & & & 0.00 & & 0.000 & 00 & 0.000 & \\
\hline $\mathrm{Ti}$ & 242 & 75 & & 0.03 & 0 & 0.000 & 0.0 & 0.000 & 0 \\
\hline$U$ & 211 & 98 & 225 & 0.25 & 57 & 0.143 & 9.3 & 0.010 & 4 \\
\hline v & 0.173 & & & 0,00 & D & 0.000 & 100.0 & 0.000 & 100 \\
\hline$W$ & & & & 0.00 & t? & 0.000 & 0.0 & 0.000 & 0 \\
\hline y & & & & 0.00 & 0 & 0.000 & 0.0 & 0.000 & \\
\hline $\mathrm{Zn}$ & 100 & & 173 & 0.19 & 0 & 0.000 & 38.0 & 0.073 & 38 \\
\hline $\mathrm{Zr}$ & 8.06 & & 20.5 & 0,02 & 0 & 0.000 & 0.0 & 0.000 & 0 \\
\hline
\end{tabular}

\begin{tabular}{|c|c|c|c|c|c|c|c|c|c|}
\hline \multirow[b]{2}{*}{ Component } & \multirow{2}{*}{$\begin{array}{r}\text { summation } \\
\text { uglg sample } \\
\text { (Rapko 1995) }\end{array}$} & \multirow[b]{2}{*}{$\begin{array}{r}\% \\
\text { recovery }\end{array}$} & \multirow[b]{2}{*}{$\begin{array}{l}\text { ugig sample } \\
\text { \{Colton 1995\} }\end{array}$} & \multirow[b]{2}{*}{$\begin{array}{r}M T \\
\{\tan k\}\end{array}$} & \multicolumn{2}{|c|}{ Wash } & \multicolumn{3}{|c|}{ Leach } \\
\hline & & & & & $\begin{array}{l}\text { \% removed } \\
\text { mitíal sollds }\end{array}$ & MT removed & $\begin{array}{r}\% \text { removed } \\
\text { washed solids }\end{array}$ & MT remaved & $\begin{array}{r}\% \text { leach } \\
\text { initial solids }\end{array}$ \\
\hline $\mathrm{OH}-$ & & & & 0.05 & & 0000 & 0.0 & 0.000 & \\
\hline $\mathrm{NO} 3-$ & 71600 & & 82000 & 91.56 & 93 & 85150 & 0.0 & 0,000 & \\
\hline $\mathrm{NO2}-$ & 46000 & & 45000 & 50.25 & 91 & 45724 & 0.0 & 0.000 & \\
\hline PO4-3 & 45600 & 103 & 45600 & 50.92 & 43 & 21.894 & 84.2 & 24.440 & 48 \\
\hline $\mathrm{SO} 4.2$ & 11100 & 77 & 11600 & 12.95 & 92 & 11.916 & 0.0 & 0.000 & \\
\hline $\mathrm{F}-$ & 950 & & 1560 & 1.74 & 100 & 1.742 & $\# D|Y| O !$ & \#DIVIO! & \\
\hline $\mathrm{Cl}$ & 1130 & & 1030 & 1.15 & 94 & 1.081 & 00 & 0.000 & \\
\hline \multirow[t]{2}{*}{$\mathrm{Br}-$} & & & & 0.00 & & 0,000 & 0.0 & 0.000 & \\
\hline & $\begin{array}{r}\text { summation } \\
\text { ucigg sample } \\
\text { (Rapko 1995) }\end{array}$ & $\begin{array}{r}\% \\
\text { recovery }\end{array}$ & $\begin{array}{r}\text { uCi/g sample } \\
\text { Colton } 1995\end{array}$ & $\begin{array}{r}c i \\
\text { (tank }\end{array}$ & $\begin{array}{l}\% \text { removed } \\
\text { initlal solids }\end{array}$ & Ci removed & $\begin{array}{r}\% \text { removed } \\
\text { washed solids }\end{array}$ & Cl removed & $\begin{array}{r}\% \text { leach } \\
\text { initial solids }\end{array}$ \\
\hline Total alpha & 0.234 & 159 & & 261.28 & 0 & 0.000 & 0.0 & 0,000 & 0 \\
\hline$P u-239,240$ & 0.11 & 965 & 0.097 & 108.31 & & 0.000 & 0.0 & 0.000 & \\
\hline$A m-241+P u-238$ & 0.063 & 138 & & 70.34 & & 0.000 & 0.0 & 0.000 & 0 \\
\hline Am-241 (gamma) & 0.081 & 25 & 0.085 & 94.91 & 0 & 0.000 & 0.0 & 0.000 & 0 \\
\hline $6 \mathrm{~mm}-243,244$ & & & & 0.00 & & 0.000 & 0.0 & 0.000 & \\
\hline Cs-137 & 140 & 103 & 158 & 176418.85 & 52 & 91737.802 & 91.7 & 77624.294 & 44 \\
\hline Co.60 & & & & 000 & & 0.000 & 0.0 & 0.000 & \\
\hline Eu-154 & & & & 0.00 & & 0.000 & 0.0 & 0.000 & \\
\hline Eu-155 & & & & 0.00 & & 0.000 & 0.0 & 0000 & \\
\hline $5 r-90$ & 240 & 105 & 496 & 553821.20 & 0 & 0.000 & 0.0 & 0.000 & 0 \\
\hline |-129 & & & & & & & 0.0 & 0.000 & \\
\hline Ic-9g & 0115 & 143 & 0.114 & 127.29 & 93 & 118.379 & 857 & 7.637 & 6 \\
\hline
\end{tabular}

Notes:

Na measurement mpractical due to melhod of sample prep (Rapko 1995). therefore, Na wash factor based on Colton 1995.

Colton. N. G. 1995. Studge Pretreatment Chemisoy Evatuation: Enhanced Studge Washing Separafion Factors, PNL-10512.

Pacific Northwest National Laboratory, Richland Washington.

Rapko, B. M. G. J. Lumetra. M. J Wagner 1995. Washing and Caustic Leaching of Hantord Tank Studges Resutts of FY 1995 Studies, PNL-10712.

Pacific Northwest Natıonal Laboratory, Richland Washington. 


\begin{tabular}{|c|c|c|c|c|c|c|c|c|c|}
\hline \multicolumn{10}{|c|}{$\begin{array}{c}1.25 \text { Density }(\mathrm{g} / \mathrm{mL}) \\
105 \text { Voturme }(\mathrm{kL})\end{array}$} \\
\hline \multirow[b]{2}{*}{ Component } & \multirow{2}{*}{$\begin{array}{r}\text { range } \\
\text { uglg sample } \\
\text { (Lumetta 1994\} }\end{array}$} & \multirow[b]{2}{*}{$\begin{array}{r}\% \\
\text { secovery }\end{array}$} & \multirow[b]{2}{*}{$\begin{array}{r}\text { ugig sample } \\
\text { (Colton 1995) }\end{array}$} & \multirow[b]{2}{*}{$\begin{array}{r}\text { MT } \\
\text { (tank) }\end{array}$} & \multicolumn{2}{|c|}{ Wash } & \multicolumn{3}{|c|}{ Leach } \\
\hline & & & & & $\begin{array}{l}\% \text { removed } \\
\text { initial solids }\end{array}$ & MT removed & $\begin{array}{l}\% \text { removed } \\
\text { washed solids }\end{array}$ & MT removed & $\begin{array}{r}\% \text { leach } \\
\text { initiad solids } \\
\end{array}$ \\
\hline $\mathrm{Ag}$ & & & & 0.00 & & 0.000 & 0 & 0.000 & \\
\hline$A .1$ & $2600-3920$ & & 4850 & 064 & 0 & 0.000 & 28 & 0180 & 28 \\
\hline As & & & & 0.00 & & 0.000 & 0 & 0000 & \\
\hline $\mathrm{B}$ & & & 65 & 0.01 & & 0.000 & 0 & 0.000 & \\
\hline $\mathrm{Ba}$ & $104 \cdot 200$ & & 396 & 0.05 & 0 & 0.000 & 0 & 0.000 & 0 \\
\hline Be & & & & 0.00 & & 0.000 & 0 & 0.000 & \\
\hline $\mathrm{Bt}$ & 72000 - 96000 & & 103400 & 13.70 & 0 & 0.000 & 0 & 0.000 & 0 \\
\hline $\mathrm{Ca}$ & $9600-12000$ & & 13800 & 1.83 & 0 & 0.000 & 1 & 0.018 & 1 \\
\hline $\mathrm{Cd}$ & & & 39.5 & 0.01 & & 0.000 & 0 & 0.000 & \\
\hline $\mathrm{Ce}$ & & & & 0.00 & & 0.000 & 0 & 0.000 & \\
\hline $\mathrm{Co}$ & & & 68.2 & 0.01 & & 0.000 & 0 & 0.000 & \\
\hline $\mathrm{Cr}$ & $2520-3160$ & & 3350 & 0.44 & 34 & 0.151 & 35 & 0.102 & 23 \\
\hline $\mathrm{Cu}$ & & & 119 & 0.02 & & 0.000 & 0 & 0,000 & \\
\hline $\mathrm{Fe}$ & $12000 \cdot 13600$ & & 15100 & 2.00 & 0 & 0.000 & 1 & 0.020 & 1 \\
\hline$k$ & & & 5990 & 0.79 & & 0.000 & 0 & 0000 & \\
\hline La & $12000-16400$ & & 15400 & 2.04 & 0 & 0.000 & 0 & 0.000 & 0 \\
\hline Li & & & & 0.00 & & 0.000 & $D$ & 0.000 & \\
\hline $\mathrm{Mg}$ & $1320 \cdot 1560$ & & 3410 & 0.45 & 0 & 0.000 & 0 & 0.000 & 0 \\
\hline $\mathrm{Mn}$ & $18000-22400$ & & 23100 & 3.06 & ( & 0.000 & 0 & 0.000 & 0 \\
\hline Mo & & & & 0.00 & & 0.000 & 0 & 0.000 & \\
\hline $\mathrm{Na}$ & $32000-40000$ & & 41500 & 5.50 & 74 & 4.068 & 0 & 0.000 & \\
\hline$N:$ & & & & 000 & & 0.000 & tr & 0.000 & \\
\hline $\mathrm{Pb}$ & $520-1200$ & & 1510 & 0.20 & 3 & 0.006 & 14 & 0.028 & 14 \\
\hline $\mathrm{Pd}$ & & & & 0.00 & & 0000 & 0 & 0.000 & \\
\hline $\mathrm{Rh}$ & & & & 0.00 & & 0.000 & 0 & 0.000 & \\
\hline Ru & & & & 0.00 & & 0.000 & 0 & 0.000 & \\
\hline $\mathrm{Sb}$ & & & & 000 & & 0.000 & 0 & 0.000 & \\
\hline Se & & & & 0.00 & & 0.000 & 0 & 0000 & \\
\hline $\mathrm{Si}$ & $12000 \cdot 20000$ & & 24300 & 3.22 & 8 & 0.258 & 47 & 9.384 & 4.3 \\
\hline Sn & & & & 0.00 & & 0.000 & 0 & 0.000 & \\
\hline$s_{r}$ & $600-840$ & & 930 & 0.12 & 0 & 0.000 & D & 0.000 & 0 \\
\hline Te & & & & 0.00 & & 0.000 & 0 & 0.000 & \\
\hline Th & & & & 0.00 & & 0.000 & D & 0.000 & \\
\hline $\mathrm{TI}$ & & & & 0.00 & 0 & 0.000 & 0 & 0.000 & 0 \\
\hline u & & & 638 & 0.08 & & 0.000 & 0 & 0.000 & \\
\hline$v$ & & & & 0.00 & & 0.000 & 0 & 0.000 & \\
\hline$w$ & & & & 0.00 & & 0.000 & 0 & 0.000 & \\
\hline$Y$ & & & & 0.00 & & 0.000 & 0 & 0.000 & \\
\hline$Z n$ & & & 278 & 0.04 & & 0.000 & 0 & 0.000 & \\
\hline $\mathrm{Zr}$ & $120 \cdot 204$ & & 62.3 & 0.01 & & 0.000 & a & 0.000 & \\
\hline
\end{tabular}

\begin{tabular}{|c|c|c|c|c|c|c|c|c|c|}
\hline \multirow[b]{2}{*}{ Component } & \multirow{2}{*}{$\begin{array}{r}\text { range } \\
\text { ug/g sample } \\
\text { (Lumetta 1994) }\end{array}$} & \multirow[b]{2}{*}{$\begin{array}{r}\% \\
\text { recovery }\end{array}$} & \multirow[b]{2}{*}{$\begin{array}{r}\text { uglg sample } \\
\text { (Colton 1995) }\end{array}$} & \multirow[b]{2}{*}{$\begin{array}{r}\text { Mr } \\
\text { (tank) }\end{array}$} & \multicolumn{2}{|c|}{ Wash } & \multicolumn{3}{|c|}{ Leach } \\
\hline & & & & & $\begin{array}{l}\% \text { removed } \\
\text { initial solids }\end{array}$ & MT removed: & $\begin{array}{l}\% \text { removed } \\
\text { wasthed solids }\end{array}$ & MT removed & $\begin{array}{l}\% \text { removed } \\
\text { init|al salids }\end{array}$ \\
\hline $\mathrm{OH}-$ & & & & 0.00 & & 0.000 & 0 & 0.000 & \\
\hline NO3- & & & 50500 & 6.69 & & 0.000 & 0 & 0.000 & \\
\hline $\mathrm{NO}_{2}-$ & & & 881 & 0.12 & & 0.000 & 0 & 0.000 & \\
\hline $\mathrm{PO} 4.3$ & $32000-40000$ & & 18200 & 2.41 & 10 & 0.241 & 18 & 0.386 & 16 \\
\hline $\mathrm{SO} 4-2$ & & & 347 & 0.05 & & 0.000 & 0 & 0,000 & \\
\hline F- & & & 6000 & 0.79 & & 0.000 & 0 & 0.000 & \\
\hline Ct- & & & 1680 & 0.22 & & $0.000 f$ & D & 0.000 & \\
\hline $\mathrm{Bi}-$ & & & & 0.00 & & 0,000 & 0 & 0.000 & \\
\hline & $\begin{array}{r}\text { range } \\
\text { uCikg sample } \\
\text { [Lumetta 1994] }\end{array}$ & $\begin{array}{r}\% \\
\text { recovery }\end{array}$ & $\begin{array}{l}\text { ucilg sample } \\
\text { (Colton 1995) }\end{array}$ & $\begin{array}{r}c i \\
(\tan k)\end{array}$ & $\begin{array}{l}\% \text { removed } \\
\text { initial solids }\end{array}$ & Ci removed & $\begin{array}{l}\% \text { removed } \\
\text { washed solids }\end{array}$ & Ci removed & $\begin{array}{l}\% \text { removed } \\
\text { imitlal solids }\end{array}$ \\
\hline Total alpha & & & & 0.00 & & 0.000 & 0 & 0.000 & \\
\hline Pu-239, 240 & & & 0.794 & 105.19 & & 0.000 & 0 & 0.000 & \\
\hline$A m-241+P u-238$ & & & & 0.00 & & 0.000 & 0 & 0.000 & \\
\hline Am-241 (gamma) & & & 0.036 & 4.77 & & 0.000 & 0 & 0000 & \\
\hline $\mathrm{Cm}-243.244$ & & & & 0.00 & & 0.000 & 0 & 0.000 & \\
\hline$C s-137$ & 016.123 & & 0.897 & 118.83 & 17 & 20.201 & 10 & 9506 & 8 \\
\hline Co-f9 & & & 0.03 & 3.97 & & 0.000 & 0 & 0.000 & \\
\hline Eu-154 & & & & 0.00 & & 0.000 & 0 & 0.000 & \\
\hline Eu-155 & & & & 0.00 & & 0.000 & 0 & 0.000 & \\
\hline Sr-90 & 32.68 & & 2.27 & 300.72 & & 0.000 & 0 & 0.000 & \\
\hline$T_{c-99}$ & $<D L$ - 0 Oro35 & & 0.002 & 0.26 & 74 & 0.196 & 0 & 0.000 & 0 \\
\hline
\end{tabular}

Notes:

Less than wash, leach values entered above as zero.

kia value not reported in Lumetta 1995. therefore. Na wash factor basod on Colton 1995.

Colton, N G 1995. Studge Fretreatment Chemisty Evaluation: Enhanced Stuotge Washing Separation Factors. PNL-10512, Paciflc Northwest National Laboratory Richiand Washington

Lumetta, G. j and B. M. Rapko. 1994. Washing and Atkatine Leaching of Hanford Situdges. A Status Report, PNL-10078.

Pacrfic Northwest National Laboratory. Richland Washington. 


\begin{tabular}{|c|c|c|c|c|c|c|c|c|c|}
\hline \multirow[b]{3}{*}{ Component } & $\begin{array}{l}1.1 \text { Density (g/mL) } \\
102 \text { Volume }(\mathrm{kL})\end{array}$ & \multirow[b]{3}{*}{$\begin{array}{r}\% \\
\text { recovery }\end{array}$} & \multirow[b]{3}{*}{$\begin{array}{l}\text { ugfg sample } \\
\text { [Appendlx) }\end{array}$} & \multirow[b]{3}{*}{$\begin{array}{r}M T \\
(\operatorname{tank})\end{array}$} & \multirow{2}{*}{\multicolumn{2}{|c|}{ Wash }} & \\
\hline & summation & & & & & & & Leach & \\
\hline & $\begin{array}{r}\text { ugig sample } \\
\text { [Jemer 1995] }\end{array}$ & & & & $\begin{array}{l}\% \text { removed } \\
\text { initial solids }\end{array}$ & MT removed & $\begin{array}{l}\% \text { removed } \\
\text { washed salids }\end{array}$ & MT removed & $\begin{array}{r}\% \text { leach } \\
\text { initial solids }\end{array}$ \\
\hline $\mathrm{Ag}$ & & & & 0.00 & & 0,000 & 0 & 0.000 & \\
\hline $\mathrm{Al}$ & 576 & 92 & 876 & 0.10 & 0 & 0.000 & 16 & 0.016 & 16 \\
\hline As & & & & 0.00 & & 0.000 & 0 & Z. 000 & \\
\hline$B$ & & & 136 & 0.02 & 90 & 0.014 & 93 & 0.001 & 9 \\
\hline $\mathrm{Ba}$ & 1060 & 101 & 1780 & 0.20 & 0 & 0.000 & 0 & 0.000 & 0 \\
\hline Be & & & & 0.00 & & 0,000 & 0 & 0.000 & \\
\hline $\mathrm{Bt}$ & 63100 & 109 & 38900 & 4.37 & 0 & 00,00 & 0 & 0.002 & 0 \\
\hline $\mathrm{Ca}$ & 1650 & & 10600 & 1.19 & 0 & 0003 & ㅁ & 0.000 & t? \\
\hline $\mathrm{Cd}$ & & & 15 & 0.00 & 0 & 0000 & 0 & 0.000 & 0 \\
\hline $\mathrm{Ce}$ & 48 & & & 0.01 & 0 & 0.000 & 0 & 0.000 & \\
\hline Co & & & 12.1 & 0.00 & 0 & 0.000 & 0 & 0000 & 0 \\
\hline $\mathrm{Cr}$ & 2450 & 91 & 3320 & 0.37 & 21 & 0.079 & 10 & 0.030 & 8 \\
\hline $\mathrm{Cu}$ & & & 40.9 & 0.00 & 0 & 0.000 & 0 & 0.000 & 0 \\
\hline $\mathrm{Fe}$ & 4940 & 96 & 6380 & 0.72 & 0 & 0.000 & 0 & 0.000 & 0 \\
\hline$K$ & 6360 & 109 & 6670 & 0.75 & 84 & 0.628 & 89 & 0.109 & 15 \\
\hline $\mathrm{La}$ & & & 14300 & 1.61 & 0 & 0000 & 0 & 0.000 & 0 \\
\hline $\mathrm{Li}$ & & & & 000 & & 0.000 & 0 & 0,000 & \\
\hline $\mathrm{Mg}$ & 276 & 107 & 539 & 0.06 & 0 & 0.000 & 0 & 0.000 & 0 \\
\hline $\mathrm{Mr}$ & 12200 & 104 & 13300 & 1.50 & 0 & 0.000 & 0 & 0.000 & 0 \\
\hline Mo & 0437 & & & 0.00 & 0 & 0.000 & 14 & 0.000 & 14 \\
\hline $\mathrm{Na}$ & 49400 & 200 & 37400 & 4.20 & 45 & 1892 & 85 & 1.976 & 47 \\
\hline $\mathrm{Ni}$ & & & 205 & 0.02 & 0 & 0000 & 0 & 0.000 & 0 \\
\hline $\mathrm{Pb}$ & 432 & 99 & 615 & 0.07 & 0 & 0.000 & 2 & 0.001 & 2 \\
\hline $\mathrm{Pd}$ & & & & 0.00 & & 0.000 & 0 & 0.000 & \\
\hline $\mathrm{Rh}$ & & & & 0.00 & & 0.000 & 0 & 0.000 & \\
\hline $\mathrm{Ru}$ & & & & 0.00 & & 0.000 & 0 & 0.000 & \\
\hline $5 b$ & & & & 0.00 & & 0000 & 0 & 0000 & \\
\hline Se & & & & 000 & & 0.000 & 0 & 0.000 & \\
\hline $\mathrm{Si}$ & 5690 & 97 & 2900 & 0.34 & 26 & 0.087 & 42 & 0.104 & 31 \\
\hline Sn & 0.876 & & & 0.00 & & 0.000 & 0 & 0.000 & \\
\hline $\mathrm{Sr}$ & 478 & 103 & 595 & 007 & 0 & 0.000 & 0 & 0.000 & 0 \\
\hline Te & & & & 0.00 & & 0.000 & 0 & 0.000 & \\
\hline Th & & & & 0.00 & & 0.000 & 0 & 0.000 & \\
\hline $\mathrm{Ti}$ & & & & 0.00 & & 0.000 & 0 & 0.000 & \\
\hline$U$ & 242 & 102 & 323 & 0.04 & 0 & 0.000 & 1 & 0.000 & 1 \\
\hline$V$ & & & & 0.00 & & 0.000 & 0 & 0.000 & \\
\hline$W$ & 3.65 & & & 0.00 & 73 & 0.000 & 69 & 0.000 & 19 \\
\hline$Y$ & & & & 0.00 & & D.000 & 0 & 0.000 & \\
\hline$Z n$ & 358 & 117 & 449 & 0.05 & 0 & 0,000 & 4 & 0.002 & 4 \\
\hline $\mathrm{Zr}$ & & & 7.66 & 0.00 & 0 & 0.000 & 0 & 0.000 & 0 \\
\hline
\end{tabular}

\begin{tabular}{|c|c|c|c|c|c|c|c|c|c|}
\hline \multirow[b]{2}{*}{ Component } & \multirow{2}{*}{$\begin{array}{l}\text { summation } \\
\text { uglg sample } \\
\text { [Temer 1995] }\end{array}$} & \multirow[b]{2}{*}{$\begin{array}{r}\% \\
\text { recovery }\end{array}$} & \multirow[b]{2}{*}{$\begin{array}{l}\text { ug/g sample } \\
\text { (Appendix\} }\end{array}$} & \multirow[b]{2}{*}{$\begin{array}{r}\text { MT } \\
\text { tank }\end{array}$} & \multicolumn{2}{|c|}{ Wash } & \multicolumn{3}{|c|}{ Leack } \\
\hline & & & & & $\begin{array}{l}\% \text { removed } \\
\text { initial solids }\end{array}$ & MT removed & $\begin{array}{r}\% \text { removed } \\
\text { washed solids }\end{array}$ & MT removed & $\begin{array}{l}\text { \% romoved } \\
\text { initial solids }\end{array}$ \\
\hline $\mathrm{OH}-$ & & & & 0.00 & & 0000 & 0 & 0.000 & \\
\hline NO3- & & & 61500 & 6.91 & & 0.000 & 0 & 0.000 & \\
\hline $\mathrm{NO}_{2}$ & & & 1090 & 0.12 & & 0.000 & 0 & 0.000 & \\
\hline POA-3 & 7030 & 98 & 8430 & 0.95 & 28 & 0.269 & 22 & 0.146 & 15 \\
\hline$\$ O A \cdot 2$ & 3170 & 90 & 2050 & 0.23 & 29 & 0067 & 26 & 0.042 & 18 \\
\hline F. & 7150 & 203 & 6240 & 070 & 14 & 0.097 & 96 & 0.580 & 83 \\
\hline $\mathrm{Cl}-$ & & & 864 & 010 & & 0000 & 0 & 0.000 & \\
\hline \multirow[t]{2}{*}{$\mathrm{Br}}$. & & & & 0.00 & & 0.000 & 0 & 0.000 & \\
\hline & $\begin{array}{r}\text { submmation } \\
\text { uCifg sample } \\
\text { [Temer 1995] }\end{array}$ & $\begin{array}{r}\% \\
\text { recovery }\end{array}$ & $\begin{array}{r}\text { uclig sample } \\
\text { [Appendix) }\end{array}$ & $\begin{array}{r}c i \\
[\tan k)]\end{array}$ & $\begin{array}{l}\% \text { removed } \\
\text { initial solids }\end{array}$ & Ci removed & $\begin{array}{r}\% \text { removed } \\
\text { washed salids }\end{array}$ & Ci removed & $\begin{array}{l}\% \text { removed } \\
\text { initial solids }\end{array}$ \\
\hline Total alpha & 0638 & 82 & & 0.00 & 0 & 0.000 & 0 & 0.000 & 0 \\
\hline Pu-239, 240 & 0.749 & 70 & 0.153 & 17.20 & 0 & 0.000 & 0 & 0.000 & 0 \\
\hline$A m-241+P_{4}-238$ & & & & 0.00 & 0 & 0.000 & 0 & 0000 & 0 \\
\hline Am-241 \{gamma & 0.0432 & 68 & 0.068 & 7.64 & & 0.000 & 0 & 0.000 & \\
\hline $\mathrm{cm}-243.244$ & & & & 0.00 & & 0.000 & 0 & 0.000 & \\
\hline $\mathrm{Cs}-137$ & 0.0288 & 109 & 0.1 & 11.24 & 52 & 5.868 & 50 & 2.700 & 24 \\
\hline Co-60 & & & 0.01 & 1.12 & & 0.000 & 0 & 0.000 & \\
\hline Eu-154 & & & & 0.00 & & 0.000 & 0 & 0.000 & \\
\hline Eu-155 & & & & 0.00 & & 0.000 & 0 & 0.000 & \\
\hline Sr-90 & 3.38 & 134 & 4.13 & 464.27 & 0 & 0.000 & 0 & 0.000 & 0 \\
\hline$T_{c-99}$ & & & 0.005 & 0.56 & 0 & 0.000 & 0 & 0.000 & 0 \\
\hline
\end{tabular}

Notes:

Characterization data worksheet for B-202 is provided in Appendix.

Jemer, D. J. and R. Villarreal. 1995. Shdige Washing and Alkatine Leaching tests on Actuat Hantord fank Siludge: A Stafus Report, LALUR 95-2070. Los Alamos National Laboratory. Los Alamos, New Mexico 
BX-105 TRB, CW Experimental Results

\begin{tabular}{|c|c|c|c|c|c|c|c|c|}
\hline \multicolumn{9}{|c|}{$\begin{array}{c}169 \text { Density }\left\{g^{f m L}\right) \\
174 \text { Volume }\{k L\}\end{array}$} \\
\hline \multirow[b]{2}{*}{ Component } & \multirow{2}{*}{$\begin{array}{r}\text { summation } \\
\text { ugig sample } \\
\text { (Temer 1995) }\end{array}$} & \multirow[b]{2}{*}{$\begin{array}{r}\% \\
\text { recovery }\end{array}$} & \multirow[b]{2}{*}{$\begin{array}{r}\text { ugig sample } \\
\text { \{Colton 1995\} }\end{array}$} & \multirow[b]{2}{*}{$\begin{array}{r}\text { MT } \\
\text { (tank) }\end{array}$} & \multicolumn{2}{|c|}{ Wash } & \multicolumn{2}{|r|}{ Leach } \\
\hline & & & & & $\begin{array}{l}\% \text { removed } \\
\text { initial solids }\end{array}$ & MT removed & $\begin{array}{r}\% \text { removed } \\
\text { washed solids }\end{array}$ & MT removed initial soljds \\
\hline $\mathrm{Ag}$ & 0.615 & & 25.8 & 0.01 & & 0.000 & 0 & 0.000 \\
\hline Al & 147000 & 112 & 33900 & 997 & & 0,000 & 0 & 0.000 \\
\hline As & & & & 0.00 & & 0.000 & 0 & 0.000 \\
\hline 6 & & & 5.3 & 0.00 & & 0.000 & 0 & 0.000 \\
\hline Bà & & & 3200 & 0.84 & & 0.000 & 0 & 0.000 \\
\hline $\mathrm{Be}$ & & & & 000 & Data from this ta & Ink have not bee & in included in this & valuation because of \\
\hline$B i$ & 4.34 & 82 & 776 & 023 & discrepancies in & projected inven & tories derived fror & core samples obtained \\
\hline $\mathrm{Ca}$ & 477 & 118 & 6210 & 183 & in 1986 and auge & er sulface samp & les obtained in 19 & 4. Auger samples may \\
\hline $\mathrm{Cd}$ & 0.834 & 140 & 44.3 & 0.01 & be representative & of of cladding wa & Iste, the last type & f waste added to this \\
\hline $\mathrm{Ce}$ & & & & 0.00 & tank. Washing a & and leaching tes & Is were performed & only on the auger \\
\hline Co & 0.394 & 72 & 0.57 & 0.00 & surface sample. & & & \\
\hline $\mathrm{Cr}$ & 154 & 79 & 8650 & 2.55 & & 0.000 & 0 & 0.000 \\
\hline $\mathrm{Cu}$ & $3 t .2$ & 74 & 27.3 & 001 & & 0.000 & 0 & 0,000 \\
\hline $\mathrm{Fe}$ & 365 & 91 & 6250 & 1.84 & & 0.000 & 0 & 0.000 \\
\hline$K$ & & & 1440 & 0.42 & & 0.000 & 0 & 0.000 \\
\hline La & & & & 0.00 & & 0.000 & 0 & 0.000 \\
\hline $\mathrm{Li}$ & & & & 0.00 & & 0.000 & 0 & 0.000 \\
\hline $\mathrm{Mg}$ & 9.93 & 99 & 3250 & 0.96 & & 0.000 & 0 & 0000 \\
\hline $\mathrm{Mn}$ & 5.03 & 44 & 1490 & 0.44 & & 0.000 & 0 & 0000 \\
\hline Mo & 14.2 & & & 0.00 & & 0.000 & 0 & 0.000 \\
\hline $\mathrm{Na}$ & 88600 & 430 & 110000 & 32.37 & & 0.000 & 0 & 0.000 \\
\hline $\mathrm{NI}$ & & & 147 & 0.04 & & 0.000 & 0 & 0.000 \\
\hline $\mathrm{Pb}$ & 48.2 & 92 & 563 & 0.17 & & 0.000 & 0 & 0.000 \\
\hline PS & & & & 0.00 & & 0.000 & 0 & 0.000 \\
\hline$R h$ & & & & 0.00 & & 0.000 & 0 & 0,000 \\
\hline$R u$ & & & & 0.00 & & 0.000 & 0 & 0.000 \\
\hline St & & & & 0.00 & & 0.000 & 0 & 0.000 \\
\hline Se & & & & 0.00 & & 0.000 & 0 & 0.000 \\
\hline $\mathrm{Si}$ & 259 & & 41500 & 12.21 & & 0,000 & 0 & 0.000 \\
\hline $\mathrm{Sn}_{n}$ & & & & 0.00 & & 0.000 & 0 & 0.000 \\
\hline Sr & & & 56.2 & 0.02 & & 0.000 & 0 & 0.000 \\
\hline Te & & & & 0.00 & & 0.000 & 0 & 0.000 \\
\hline Th & & & & 0.00 & & 0.000 & 0 & 0.000 \\
\hline $\mathrm{Ti}$ & & & & 0.00 & & 0.000 & 0 & 0.000 \\
\hline$\cup$ & 172 & & 4410 & 1.30 & & 0.000 & 0 & 0.000 \\
\hline$v$ & & & & 0.00 & & 0.000 & 0 & 0.000 \\
\hline W & & & & 0.00 & & 0.000 & 0 & 0.000 \\
\hline$Y$ & & & & 0.00 & & 0.000 & 0 & 0.000 \\
\hline Zn & 16.1 & & 143 & 0.04 & & 0.000 & 0 & 0.000 \\
\hline $\mathrm{Zr}$ & 1.46 & & 335 & 0.10 & & 0.000 & 0 & 0,000 \\
\hline
\end{tabular}

\begin{tabular}{|c|c|c|c|c|c|c|c|c|c|}
\hline \multirow[b]{2}{*}{ Component } & \multirow{2}{*}{$\begin{array}{r}\text { summation } \\
\text { ugig sample } \\
\text { (Temer 1995) }\end{array}$} & \multirow[b]{2}{*}{$\begin{array}{r}\% \\
\text { recovery }\end{array}$} & \multirow[b]{2}{*}{$\begin{array}{l}\text { ug/g sample } \\
\text { (Colton } 1955 \text { ) }\end{array}$} & \multirow[b]{2}{*}{$\begin{array}{r}\text { MT } \\
\text { [tank] }\end{array}$} & \multicolumn{2}{|c|}{ Wash } & \multicolumn{3}{|c|}{ Leack } \\
\hline & & & & & $\begin{array}{l}\text { \% remaved } \\
\text { initial solids }\end{array}$ & MT removed & $\begin{array}{l}\% \text { removed } \\
\text { washed solids }\end{array}$ & MT removed & $\begin{array}{l}\text { \% removed } \\
\text { initlal solids }\end{array}$ \\
\hline $\mathrm{OH}-$ & & & 3600 & 1.06 & & 0.000 & 0 & 0.000 & \\
\hline $\mathrm{NOS}-$ & & & 33250 & 9.78 & & 0.000 & 0 & 0.000 & \\
\hline $\mathrm{NO} 2$. & & & & 0.00 & & 0.000 & 0 & 0.000 & \\
\hline POA-3 & 1860 & & 59700 & 17.57 & & 0.000 & 0 & 0.000 & \\
\hline $504 \cdot 2$ & & & & 0.00 & & 0.000 & 0 & 0.000 & \\
\hline $\mathrm{F}$ - & & & & 000 & & 0.000 & 0 & 0.000 & \\
\hline C1- & & & & 0.00 & & 0.000 & 0 & 0.000 & \\
\hline \multirow[t]{2}{*}{$\mathrm{Br}-$} & & & & 0.00 & & 0.000 & 0 & 0,000 & \\
\hline & $\begin{array}{l}\text { summation } \\
\text { ucijg sample } \\
\text { (Temer 1995) }\end{array}$ & $\begin{array}{r}\% \\
\text { recovery }\end{array}$ & $\begin{array}{l}\text { uCijg sample } \\
\text { (Colton 1995) }\end{array}$ & $\begin{array}{r}C i \\
{[\tan k]}\end{array}$ & $\begin{array}{l}\text { \% removed } \\
\text { initial solids }\end{array}$ & Ci removed & $\begin{array}{l}\% \text { removed } \\
\text { washed solids }\end{array}$ & Ci removed & $\begin{array}{l}\% \text { removed } \\
\text { initlal sollds }\end{array}$ \\
\hline Total alpha & & & & 000 & & 0.000 & 0 & 0.000 & \\
\hline Pu-239, 240 & 0.499 & & 0.28 & 82.39 & & 0.000 & 0 & 0.000 & \\
\hline$A_{m}-241+P_{u-2} 36$ & & & & 000 & & 0.000 & 0 & 0.000 & \\
\hline Am-241 tgamma\} & 0.0086 & & 0.994 & 292.48 & & 0000 & 0 & 0.000 & \\
\hline $\mathrm{Cm}-243.244$ & & & & 0.00 & & 0.000 & 0 & 0.000 & \\
\hline Cs -137 & 25.1 & & 54.9 & 15918.70 & & 0.000 & 0 & 0.000 & \\
\hline Co-60 & & & 0.157 & 46.20 & & 0.000 & 0 & 0.000 & \\
\hline Eu-154 & & & & 0.00 & & 0.000 & 0 & 0.000 & \\
\hline Eu. 155 & & & & 0.00 & & 0.000 & 0 & 0.000 & \\
\hline Sir-90 & 1.3 & & 137 & 40311.69 & & 0000 & 0 & 0.000 & \\
\hline Te. 99 & & & & 0.00 & & 0.000 & 0 & 0.000 & \\
\hline
\end{tabular}

\section{Notes:}

Colton. N. G. 1995. Siudge Fretreatment Chemisty Evatuation: Enhanced Studge Wasting Separation Factors, PNL-10512.

Pacific Northwest National Laboratory, Richland Washington

Temer. D. I anj R. Villarreal. 1995. Siudge Washing and Alkaltine Leaching Tests on Actual Hantord Jank Situdge: A Status Report, LAUR 95-2070, Los Ałamos National Laborratory. Los Alamos. New Mexico 


\begin{tabular}{|c|c|c|c|c|c|c|c|c|c|}
\hline \multicolumn{10}{|c|}{$\begin{array}{r}1.2 \text { Density (gimL) } \\
1302 \text { Volume }(\mathrm{kL}) \\
\end{array}$} \\
\hline \multirow[b]{2}{*}{ Component } & \multirow{2}{*}{$\begin{array}{r}\text { summation } \\
\text { ugig sample } \\
\text { (Rapko 1995) }\end{array}$} & \multirow[b]{2}{*}{ recovery } & \multirow[b]{2}{*}{$\begin{array}{r}\text { ugig sample } \\
\text { (Colton 1995) }\end{array}$} & \multirow[b]{2}{*}{$\begin{array}{r}M T \\
(\tan k+)\end{array}$} & \multicolumn{2}{|c|}{ Wash } & \multicolumn{3}{|c|}{ Leach } \\
\hline & & & & & $\begin{array}{l}\% \text { removed } \\
\text { initial solids }\end{array}$ & MT removed & $\begin{array}{r}\% \text { removed } \\
\text { Washed solids }\end{array}$ & MT removed & $\begin{array}{r}\% \text { leach } \\
\text { initial soltds } \\
\end{array}$ \\
\hline$\overline{A g}$ & & & & 0.07 & & 0.000 & 0 & 0.000 & \\
\hline AI & 12300 & 81 & 14300 & 2234 & 1 & 0.223 & 68 & 14.970 & 67 \\
\hline A5 & 171 & & & 0.03 & 0 & 0.000 & 100 & 0,027 & 100 \\
\hline$B$ & & & 47.1 & 007 & 5 & 0.004 & 1 & 0.001 & 1 \\
\hline Bà & 567 & & 9.14 & 0.01 & 0 & 0.000 & 0 & 0.000 & 0 \\
\hline $\mathrm{Be}$ & & & & 0.00 & & 0.000 & 0 & 0.000 & \\
\hline $8 i$ & 33100 & 159. & 24100 & 37.65 & 0 & 0.000 & 0 & 0.000 & 0 \\
\hline $\mathrm{Ca}$ & 194 & 22 & 1040 & 1.62 & 14 & 0.227 & 16 & 0.227 & 14 \\
\hline $\mathrm{Cd}$ & 2.84 & & 4.2 & 0.01 & 0 & 0,000 & 0 & 0.000 & 0 \\
\hline $\mathrm{Ce}$ & 84.9 & & & 0.13 & 0 & 0.000 & 0 & 0.000 & 0 \\
\hline Co & 2.84 & 13 & 6.94 & 0.01 & 0 & 0.000 & 0 & 0.000 & 0 \\
\hline $\mathrm{Cr}$ & 585 & 59 & 968 & 1.51 & 21 & 0.318 & 10 & 0.121 & 8 \\
\hline Cu & 9.87 & 3 & 51.7 & 0.08 & 0 & 0.000 & 4 & 0.003 & 4 \\
\hline $\mathrm{Fe}$ & 6840 & 56 & 11160 & 17.34 & 0 & 0.000 & 0 & 0.000 & 0 \\
\hline$k$ & & & 263 & 0.41 & & 0.000 & 0 & 0.000 & \\
\hline La & & & & 0.0 . & & 0.000 & 0 & 0.000 & \\
\hline LI & & & & 0.00 & & 0.000 & 0 & 0.000 & \\
\hline $\mathrm{Mg}$ & 84.9 & 49 & 159 & 0.25 & 0 & 0000 & 0 & 0.000 & 0 \\
\hline $\mathrm{Mn}$ & 38 & 20 & 64.6 & $\begin{array}{lll}0 & 10\end{array}$ & to & 0.010 & 1 & 0.001 & 1 \\
\hline Mo & 15 & & & 002 & 69 & 0.016 & 0 & 0.000 & 0 \\
\hline Na & & & 101000 & 157.81 & 67 & 105.731 & 0 & 0.000 & \\
\hline $\mathrm{Ni}$ & & & 12.3 & 0.02 & & 0.000 & 0 & 0.000 & \\
\hline $\mathrm{Pb}$ & 56.7 & & 74.5 & 0.12 & 0 & 0.000 & 0 & 0.000 & 0 \\
\hline $\mathrm{Pd}$ & & & & 0.00 & & 0,000 & 0 & 0000 & \\
\hline Rh & & & & 0.00 & & 0.000 & 0 & 0,000 & \\
\hline Rú & & & & 0.00 & & 0.000 & 0 & 0.000 & \\
\hline So & & & & 0.00 & & 0.000 & 0 & 0.000 & \\
\hline Se & 18.8 & & & 0.03 & 0 & 0.000 & 0 & 0.000 & 0 \\
\hline \$i & 4760 & 55 & 6780 & 10 59. & 0 & 0.000 & 3 & 0.318 & 3 \\
\hline Sn & & & & 0.00 & & 0.000 & 0 & 0.000 & \\
\hline Sr & 88.8 & 55 & 169 & 0.26 & 0 & 0.000 & 0 & 0.000 & 0 \\
\hline $\mathrm{Te}$ & & & & 000 & & 0.000 & 0 & 0.000 & \\
\hline Th & & & & 0.00 & & 0.000 & 0 & 0.000 & \\
\hline$T_{1}$ & 6.62 & 38 & & 0.01 & 0 & 0.000 & 0 & 0.000 & 0 \\
\hline$U$ & 1990 & 32 & 2270 & 3.55 & +6 & 0.567 & 5 & 0.142 & 4 \\
\hline$v$ & & & & 0.00 & & 0.000 & 0 & 0.000 & \\
\hline$W$ & & & & 0.00 & & 0.000 & 0 & 0.000 & \\
\hline$Y$ & & & & 0.00 & & 0.000 & 0 & 0.000 & \\
\hline Zn & 65.2 & 30 & 83.4 & 0.13 & 0 & 0.000 & 57 & 0.074 & 57 \\
\hline $\mathrm{Zr}$ & 818 & & 136 & 0.21 & 0 & 0.000 & 1 & 0.002 & 1 \\
\hline & stummation & & & & Wa: & & & Leach & \\
\hline Camponent & $\begin{array}{r}\text { Hylg sample } \\
\text { (Rapka 1995) }\end{array}$ & $\begin{array}{r}\% \\
\text { recovery }\end{array}$ & $\begin{array}{r}\text { uglg sample } \\
\text { (Colton 1995) }\end{array}$ & $\underset{(\tan k)]}{\mathbf{M T}}$ & $\begin{array}{l}\text { \% remaved } \\
\text { initial solids }\end{array}$ & MT rimaved & $\begin{array}{r}\% \text { removed } \\
\text { washed solids }\end{array}$ & MT removed & $\begin{array}{l}\% \text { removid } \\
\text { initial solids }\end{array}$ \\
\hline OH. & & & & 0.00 & & 0.000 & 0 & 0.000 & \\
\hline NO3- & 90100 & & 137000 & 214.06 & 95 & 203.353 & 100 & 10.703 & 5 \\
\hline $\mathrm{NO} 2-$ & 17600 & & 8130 & 12.70 & 88 & 11.178 & 100 & 1.524 & 12 \\
\hline $\mathrm{PO} 4.3$ & 65600 & 92 & 70400 & 110.00 & 20 & 21.999 & 91 & 80.297 & 73 \\
\hline so4-2 & 9790 & 114. & 13700 & 21.41 & 90 & 19.265: & 50 & 1.070 & 5 \\
\hline F- & 14600 & & 9080 & 14.19 & 23 & 3.263 & 99 & 10.782 & 76 \\
\hline $\mathrm{Cl}$ & 905 & & 1150 & 1.80 & 94 & 1.689 & 100 & 0.108 & 6 \\
\hline \multirow[t]{2}{*}{ Br- } & & & & 0.00 & & 0.000 & 0 & 0.000 & \\
\hline & $\begin{array}{r}\text { summation } \\
\text { uciig sample } \\
\text { (Rapko f995) }\end{array}$ & recovery & $\begin{array}{l}\text { uCilg sample } \\
\text { (Colton } 1995 \text { ) }\end{array}$ & $\begin{array}{r}c i \\
\tan k\}\end{array}$ & $\begin{array}{l}\% \text { romoved } \\
\text { initial solids }\end{array}$ & Ci ramoved & $\begin{array}{r}\% \text { ramoved } \\
\text { washed solids }\end{array}$ & Cl removed & \% removed \\
\hline Total alpha & 0133 & 89 & & 207.89 & 0 & 0.000 & 0 & 0.000 & 0 \\
\hline Pu-239, 240 & 0.119 & 89 & 0.057 & 89.06 & & 0.000 & 0 & 0.000 & \\
\hline A.m-241+Pu-238 & 0.0137 & 98 & & 21.41 & & 0.000 & 0 & 0.000 & \\
\hline Am-241 igammai & 0.0118 & & 0.018 & 28.12 & 0 & 0.000 & 0 & 0.000 & 0 \\
\hline $\mathrm{Cm}-243,244$ & & & & 0.00 & & 0.000 & 0 & 0.000 & \\
\hline $\mathrm{Cs}-137$ & 14.2 & 86 & 17.4 & $27 \nmid 86.60$ & 17 & 4621.721 & 93 & 20933.678 & 77 \\
\hline $60-60$ & & & & 0.00 & & 0.000 & 0 & 0.000 & \\
\hline Eu-154 & & & & 0.00 & & 0.000 & 0 & 0.000 & \\
\hline Eu-155 & & & & 0.00 & & 0.000 & 0 & 0.000 & \\
\hline $5 r-90$ & 7.19 & 72 & 9.87 & 1542136 & 0 & 0.000 & 0 & 0.000 & 0 \\
\hline Tc-99 & 0.0314 & 140 & 0.037 & 57.81 & 92 & 53.186 & 88 & 4.047 & 7 \\
\hline
\end{tabular}

Notes

Na measurement impractical due to method of sampie prep (Rapko 1995); therefore. Na wash factor based on Colton 1995.

Colton. N G 1995. Situdge Pretrealment Chemistry Evatuation Enhanced Siudge Washing Separation Factors, PNL-10512,

Pacific Northwest National Laboratory, Richland Washington

Rapko, B M. G J Lumette. M. J Wagner 1995 Washing and Caustsc Leachirig of Hantord Tank Sfudges Results of FY1995 Studies, PNL-10712,

Pacific Northwest National Laboratory, Rachland Washington 
$B X-109(T B P / C W)$ Experimental Results

\begin{tabular}{|c|c|c|c|c|c|c|c|c|c|}
\hline \multicolumn{2}{|c|}{$\begin{array}{r}1.5 \text { Density }(\mathrm{g} / \mathrm{mL}) \\
731 \text { Volume }(\mathrm{ki})\end{array}$} & \multirow[b]{3}{*}{$\%$} & \multirow[b]{3}{*}{$\begin{array}{l}\text { ugig sample } \\
\text { (Appendix) }\end{array}$} & \multirow[b]{3}{*}{$\begin{array}{r}M T \\
\text { \{tank\} }\end{array}$} & \multirow{2}{*}{\multicolumn{2}{|c|}{ Wash }} & \\
\hline \multirow[b]{2}{*}{ component } & \multirow{2}{*}{$\begin{array}{r}\text { summation } \\
\text { ug/s sample } \\
\text { (Temer } 1996 \text { ) }\end{array}$} & & & & & & & Leach & \\
\hline & & & & & $\begin{array}{l}\text { \% remaved } \\
\text { initial solids }\end{array}$ & MT removed & $\begin{array}{r}\% \text { removed } \\
\text { washed solids }\end{array}$ & MT removed & $\begin{array}{l}\% \text { leach } \\
\text { initial solids }\end{array}$ \\
\hline $\mathrm{Ag}$ & & & & $\frac{1.00}{0.00}$ & & 0.000 & 0 & 0.000 & \\
\hline$\left.A\right|^{3}$ & 555 & 81 & 1910 & 2.09 & 61 & 1.277 & 93 & 0.760 & 36 \\
\hline As & 5.7 & $2 B$ & & 001 & 10 & 0.001 & 25 & 0.001 & 23 \\
\hline$B$ & 6.45 & 74 & & 001 & 1 머을 & 0.007 & \#DIVIO! & \#D|VIO! & \\
\hline Ba & 36.1 & 87 & & 0.04 & 0 & 0000 & 0 & 0.000 & 0 \\
\hline $\mathrm{Be}$ & & & & 0.0 & & 0000 & 0 & 0.000 & \\
\hline $\mathrm{Bi}$ & 396 & 78 & & 0.43 & 0 & 0.000 & 0 & 0.001 & 0 \\
\hline $\mathrm{Ca}$ & 2170 & 99 & 4030 & 442 & 0 & 0.000 & 0 & 0.000 & 0 \\
\hline $\mathrm{Cd}$ & & & & 0.00 & & 0.000 & 0 & 0.000 & \\
\hline $\mathrm{Ce}$ & & & & 0.00 & & 0.000 & 0 & 0.000 & \\
\hline$c_{0}$ & & & & 0.00 & & 0.000 & 0 & 0.000 & \\
\hline $\mathrm{Cr}$ & 133 & 78 & 137 & 0.15 & 36 & 0.054 & 70 & 0.067 & 45 \\
\hline $\mathrm{Cu}$ & 16.1 & 78 & & 0.02 & 0 & 0.000 & 7 & 0.001 & 7 \\
\hline $\mathrm{Fe}$ & 17500 & 85 & 21500 & 23.56 & 0 & 0.000 & 0 & 0.047 & 0 \\
\hline k & 307 & 121 & & 0.00 & 66 & 0.000 & 81 & 0.000 & 28 \\
\hline La & 8.9 & 98 & & 0.01 & 0 & 0.000 & 0 & 0.000 & 0 \\
\hline Lı & & & & 0.00 & & 0.000 & 0 & 0.000 & \\
\hline $\mathrm{Mg}$ & 411 & 96 & 748 & 0.82 & 0 & 0.000 & 0 & 0.000 & 0 \\
\hline Mn & 96 & 39 & & 0.11 & 0 & 0.000 & 0 & 0.000 & 0 \\
\hline Mo & & & & 0.00 & & 0.000 & 0 & 0.000 & \\
\hline $\mathrm{Na}$ & (1000000 direct) & & 114000 & 12492 & 96 & $1 \uparrow 9.920$ & 0 & 0.000 & \\
\hline $\mathrm{Nu}$ & 51.5 & 94 & & 0.06 & 1 & 0.001 & 0 & 0.000 & \\
\hline Po & 4135 & 94 & 668 & 0.73 & 0 & 0.000 & 3 & 0.023 & 3 \\
\hline $\mathrm{Pd}$ & & & & 0.00 & & 0000 & 0 & 0.000 & \\
\hline $\mathrm{Rh}$ & & & & 0.00 & & 0.000 & a & 0.000 & \\
\hline Ru & & & & 0.00 & & 0.000 & 0 & 0.000 & \\
\hline $\mathrm{sb}$ & & & & 000 & & 0.000 & 0 & 0.000 & \\
\hline se & & & & 000 & & 0.000 & 0 & 0.000 & \\
\hline $\mathrm{Si}$ & 535 & 123 & 1720 & 1.88 & 12 & 0.226 & 24 & 0.396 & 21 \\
\hline Sn & & & & 0.00 & & 0.000 & 0 & $0.00 \mathrm{~T}$ & \\
\hline $\mathrm{Sr}$ & 540 & 96 & 590 & 0.65 & 0 & 0.000 & 0 & 0.001 & 0 \\
\hline Te & & & & 0.00 & & 0.000 & 0 & 0.000 & \\
\hline$T h$ & & & & 0.00 & & 0.000 & 0 & 0.000 & \\
\hline$T_{1}$ & & & & 0.00 & & 0.000 & 0 & 0.000 & \\
\hline U & 16200 & 81 & t5200 & 17.75 & 2 & 0.355 & 0 & 0.000 & \\
\hline V & & & & 0.00 & & 0.000 & 0 & 0.000 & \\
\hline w & & & & 0.00 & & 0.000 & 0 & 0.000 & \\
\hline$Y$ & & & & 0.00 & & 0.000 & 0 & 0.000 & \\
\hline $\mathrm{Zn}$ & 95.5 & 90 & 624 & 0.68 & 0 & 0.000 & 44 & 0.302 & 44 \\
\hline $\mathrm{Zr}$ & 606 & 90 & & 0.66 & 0 & 0.000 & 1 & 0.006 & 1 \\
\hline
\end{tabular}

\begin{tabular}{|c|c|c|c|c|c|c|c|c|c|}
\hline \multirow[b]{2}{*}{ Component } & \multirow{2}{*}{$\begin{array}{r}\text { summation } \\
\text { ugfg sample } \\
\text { (Temer 1996) }\end{array}$} & \multirow[b]{2}{*}{$\begin{array}{r}\% \\
\text { recovery }\end{array}$} & \multirow[b]{2}{*}{$\begin{array}{l}\text { ug/g sample } \\
\text { [appendl|x] }\end{array}$} & \multirow[b]{2}{*}{$\begin{array}{r}\text { MT } \\
\operatorname{tank} \mid\end{array}$} & \multicolumn{2}{|c|}{ Wash } & \multicolumn{3}{|c|}{ Leach } \\
\hline & & & & & $\begin{array}{l}\text { \% removed } \\
\text { initial solids }\end{array}$ & MT remaved: & $\begin{array}{r}\text { \%o removed } \\
\text { washed solids }\end{array}$ & MT remoyed & $\begin{array}{l}\text { \% removed } \\
\text { initial solids }\end{array}$ \\
\hline $\mathrm{OH}-$ & & & & 0.00 & & 0.060 & 0 & 0.000 & \\
\hline NO3- & & & 213000 & 233.40 & & 0.000 & 0 & 0.000 & \\
\hline NO2. & & & 19100 & 20.93 & & 0.000 & 0 & 0.000 & \\
\hline $\mathrm{PO} 4-3$ & & & 66000 & 72.32 & 76 & 54.963 & 83 & 14.464 & 20 \\
\hline $\mathrm{SO} 4-2$ & & & 18800 & 20.60 & & 0.000 & 0 & 0.000 & \\
\hline $\mathrm{F}$ - & & & $<500$ & \#VALUE! & & \#VALUE! & 0 & maLUE! & \\
\hline a. & & & 1200 & 1.31 & & 0.000 & 0 & 0.000 & \\
\hline Br- & & & & 0.00 & & 0.000 & 0 & 0.000 & \\
\hline & $\begin{array}{r}\text { summation } \\
\text { ucifg sample } \\
\text { (Temer 1996] }\end{array}$ & $\begin{array}{r}\% \\
\text { recovery }\end{array}$ & $\begin{array}{l}\text { ucilg sample } \\
\text { (Apprinndix) }\end{array}$ & $\begin{array}{r}\mathbf{C i} \\
{[\tan \mathbf{k}\}}\end{array}$ & $\begin{array}{l}\% \text { removed } \\
\text { initial sollds }\end{array}$ & Cl removed: & $\begin{array}{l}\text { \% removed } \\
\text { washed sollds }\end{array}$ & Ci removed & $\begin{array}{l}\% \text { removed } \\
\text { initial solids }\end{array}$ \\
\hline Total alpha & 0.05 & 66 & $<0.05$ & 54.79 & & 0,000 & $\overline{0}$ & 0.000 & 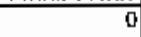 \\
\hline$P u=239,240$ & 0.033 & 108 & & 0.00 & & 0.000 & 0 & 0.000 & \\
\hline$A m-241+P u-238$ & 0.0013 & 75 & & 0.00 & & 0.000 & 0 & 0.000 & \\
\hline Am-241 (gamma) & & & 41.26 & "VALUE! & & HVALUE! & 0 & AVALUE! & \\
\hline $\mathrm{Cm}-243,244$ & & & & 0.00 & & 0.000 & 0 & 0.000 & \\
\hline Cs-137 & 12.8 & 101 & 13.9 & 15231.03 & & 0.000 & 100 & 15.185 .336 & 100 \\
\hline$C 0-60$ & & & $<0.13$ & NALUE! & & \$NALUE! & 0 & NALUE! & \\
\hline Eu-154 & & & & 0.00 & & 0.000 & 0 & 0.000 & \\
\hline Eu-155 & & & & 0.00 & & 0.000 & 0 & 0.000 & \\
\hline Sr.90 & 154 & 92.4 & 178 & 195044.84 & & 0.000 & 0 & 0.000 & 0 \\
\hline TC-99 & $<0.03$ & & & 0.00 & & 0.000 & 0 & 0.000 & \\
\hline
\end{tabular}

Notes:

Characterization trała worksheet in Appendix.

Temer. D J. and R. Vallarreal 1996 Sifuge Washing and Afkaline Leaching Tests on Acluat Manford Tank Sludge: Fy 1996 Results. LALUR96-2839. Los Alamos National Laboratory. Los Alamo5, New Mexico 
BY-104 [TBP\{0, EB]

\begin{tabular}{|c|c|c|c|c|c|c|c|c|c|}
\hline \multicolumn{10}{|c|}{\begin{tabular}{|c|}
1.6 Density $(g / m L)$ \\
1537 volume $(k L)$ \\
\end{tabular}} \\
\hline \multirow[b]{2}{*}{ Component } & \multirow{2}{*}{$\begin{array}{r}\text { summation } \\
\text { ug/g sample } \\
\text { Lumetta 1996 }\end{array}$} & \multirow[b]{2}{*}{$\begin{array}{r}\% \\
\text { recovery }\end{array}$} & \multirow[b]{2}{*}{$\begin{array}{l}\text { ug'g sample } \\
\text { (Appendix) }\end{array}$} & \multirow[b]{2}{*}{$\begin{array}{r}M T \\
{[\operatorname{tank}]}\end{array}$} & \multicolumn{2}{|c|}{ Wash } & \multicolumn{3}{|c|}{ Leach } \\
\hline & & & & & $\begin{array}{l}\text { \% removed } \\
\text { initial solids }\end{array}$ & MT removed & $\begin{array}{r}\% \text { removed } \\
\text { washed solids }\end{array}$ & MT removed & $\begin{array}{r}\text { \% leach } \\
\text { initial solids }\end{array}$ \\
\hline $\mathrm{Ag}$ & & & & 000 & & 0.000 & 0 & 0.000 & \\
\hline At & 14500 & 93 & 47900 & 11777 & 66 & 77730 & 97 & 38.865 & 33 \\
\hline As & & & 31 & 0.08 & & 0000 & 0 & 0.000 & \\
\hline $\mathrm{B}$ & & & & 0.00 & & 0.000 & 0 & 0.000 & \\
\hline $\mathrm{Ba}$ & & & & 0.00 & & 0.000 & 0 & 0.000 & \\
\hline Be & & & & 000 & & 0.000 & 0 & 0000 & \\
\hline$B_{1}$ & $\angle \mathrm{BOW}$ & & 79.3 & 0.19 & & 0.000 & 0 & 0.000 & \\
\hline $\mathrm{Ca}$ & 350 & 10 & 1110 & 2.73 & & 0.000 & 0 & 0.000 & \\
\hline Cd & 27.4 & & 69.5 & 0.17 & & $0.00 \mathrm{ar}$ & 0 & 0.000 & \\
\hline $\mathrm{Ce}$ & & & & 0.00 & & 0.000 & 0 & 0.000 & \\
\hline Co & 16.6 & & 36.5 & 0.09 & & 0.000 & 0 & 0.000 & \\
\hline $\mathrm{Cr}$ & 5670 & 145 & 5800 & 14.26 & 70 & 9.982 & 3 & 0.143 & 1 \\
\hline $\mathrm{Cu}$ & 5.36 & & & 0.00 & & 0.000 & 0 & 0,000 & \\
\hline $\mathrm{Fe}$ & 1700 & & 4790 & 11.78 & & 0.000 & 0 & 0.000 & \\
\hline K. & 3060 & 32 & 2650 & 6,52 & 95 & 6.190 & 0 & 0.000 & \\
\hline La & & & & 0.00 & & 0.000 & 0 & 0.000 & \\
\hline Li & & & & 0.00 & & 0.000 & 0 & 0.000 & \\
\hline $\mathrm{Mg}$ & & & & 0.00 & & 0.000 & 0 & 0.000 & \\
\hline Mn & 89.3 & 34 & & 0.22 & 0 & 0.000 & 0 & 0.000 & \\
\hline Mo & & & & 0.00 & & 0.000 & 0 & 0.000 & \\
\hline $\mathrm{Na}$ & 250000 & 107 & 225000 & 553.22 & 100 & 553.216 & HDIVOF & HONIO! & \\
\hline $\mathrm{Ni}_{\mathrm{I}}$ & & & 920 & 2.26 & & 0.000 & 0 & 0.000 & \\
\hline $\mathrm{Pb}$ & 179 & & & 0.00 & 14 & 0.000 & 0 & 0.000 & \\
\hline $\mathrm{Pd}$ & & & & 0.00 & & 0.000 & 0 & 0.000 & \\
\hline Rh & & & & 0.00 & & 0.000 & a & 0.000 & \\
\hline Ru & & & & 0.00 & & 0.000 & 0 & 0.000 & \\
\hline$S b$ & & & & 0.00 & & 0.000 & 0 & 0,000 & \\
\hline Se & & & & 0.00 & & 0.000 & 0 & 0.000 & \\
\hline $\mathrm{Si}$ & 610 & 37 & 1494 & 367 & 46 & $\uparrow .690$ & 37 & 0.735 & 20 \\
\hline Sn & & & & 0.00 & & 0.000 & 0 & 0.000 & \\
\hline Sr & 380 & 113 & 1160 & 285 & & 0.000 & 0 & 0.000 & \\
\hline $\mathrm{Te}$ & & & & 0.00 & & 0.000 & 0 & 0.000 & \\
\hline$T h$ & & & & 0.00 & & 0.000 & 0 & 0.000 & \\
\hline $\mathrm{TI}_{1}$ & & & & $0 . \mathrm{WH}$ & & 0.000 & 0 & 0.000 & \\
\hline U & & & 2680 & 8.59 & & 0.000 & 0 & 0.000 & \\
\hline V & & & & 0.00 & & 0.000 & a & 0.000 & \\
\hline$w$ & & & & 0.00 & & 0.000 & 0 & 0.000 & \\
\hline$Y$ & & & & 0.00 & & 0,000 & 0 & 0.000 & \\
\hline $2 n$ & 20.5 & & 250 & 0.61 & 0 & 0.000 & 31 & 0.191 & 31 \\
\hline$Z_{r}$ & 14 & & 53 & 0.13 & 0 & 0.000 & 0 & 0.000 & 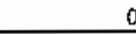 \\
\hline
\end{tabular}

\begin{tabular}{|c|c|c|c|c|c|c|c|c|c|}
\hline \multirow[b]{2}{*}{ Component } & \multirow{2}{*}{$\begin{array}{r}\text { summation } \\
\text { ug/g sample } \\
\text { (Lumetta t996) }\end{array}$} & \multirow[b]{2}{*}{$\begin{array}{r}\% \\
\text { recovery }\end{array}$} & \multirow[b]{2}{*}{$\begin{array}{l}\text { ug/g sample } \\
\text { (Appendix) }\end{array}$} & \multirow[b]{2}{*}{$\begin{array}{r}\text { MT } \\
\text { [tank] }\end{array}$} & \multicolumn{2}{|c|}{ Wash } & \multicolumn{3}{|c|}{ Leach } \\
\hline & & & & & $\begin{array}{l}\% \text { removed } \\
\text { Inltial sollds }\end{array}$ & MT removed & $\begin{array}{l}\% \text { removed } \\
\text { washed solids }\end{array}$ & MT removed & $\begin{array}{l}\text { \% removec } \\
\text { initial solid }\end{array}$ \\
\hline $\cos (2-) ? ?$ & & & 191000 & 469.62 & & 0.000 & 0 & 0.000 & \\
\hline $\mathrm{NO} 3-$ & & & 61600 & 151.95 & & 0000 & 0 & 0.000 & \\
\hline NO2. & & & 13800 & 33.93 & & 0.000 & 0 & 0.000 & \\
\hline PO4-3 & 9860 & 138 & 15700 & 3860 & 94 & 36.286 & 17 & 0.386 & 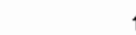 \\
\hline $504-2$ & & & 35500 & 87.29 & & 0000 & 0 & 0,000 & \\
\hline$F-$ & & & 9420 & 2316 & & 0.000 & 0 & 0.000 & \\
\hline $\mathrm{Cl}-$ & & & 1560 & 384 & & 0.000 & 0 & 0.000 & \\
\hline Br- & & & & 0.00 & & 0.000 & 0 & 0.000 & \\
\hline & $\begin{array}{r}\text { summation } \\
\text { uciig sample } \\
\text { Lumetta 1996) }\end{array}$ & $\begin{array}{r}\% \\
\text { recovery }\end{array}$ & ucilg sample & $\begin{array}{r}\mathrm{Ci} \\
\text { itank\} }\end{array}$ & $\begin{array}{l}\% \text { rumoved } \\
\text { initial solids }\end{array}$ & Ci removed & $\begin{array}{l}\alpha_{6} \text { removed } \\
\text { washed sollds }\end{array}$ & Ci removed & $\begin{array}{l}\% \text { removes } \\
\text { |nit|al solid }\end{array}$ \\
\hline Total alpha & & & & 0.00 & & 0.000 & 0 & 0.000 & \\
\hline Pu-239.240 & & & & 0.00 & & 0.000 & 0 & 0.000 & \\
\hline$A m=241+P u-23 B$ & & & & 000 & & 0.000 & 0 & 0.000 & \\
\hline A Am-241 (gamma) & & & & 0.00 & & 0.000 & 0 & 0.000 & \\
\hline $\mathrm{Cm}-243244$ & & & & 0.00 & & 0.000 & 0 & 0.000 & \\
\hline Cs.137 & & & & 0.00 & & 0.000 & 0 & 0.000 & \\
\hline 00.60 & & & & 0.00 & & 0.000 & 0 & 0.000 & \\
\hline Eu-154 & & & & 0.00 & & 0.000 & 0 & 0.000 & \\
\hline Eu-155 & & & & 0.00 & & 0.000 & 0 & 0.000 & \\
\hline $\mathrm{Sr}-90$ & & & & 0.00 & & 0.000 & 0 & 0.000 & \\
\hline Tc-99 & & & & 0.00 & & 0.000 & 0 & 0.000 & \\
\hline
\end{tabular}

Notes:

Characterizatıon data worksheet in Appendix.

[CO3] consistent with BY-110 (noticeably high) was added to ion balance

Radionuclides not reponed.

Lumetta G J. B. M. Rapko, M. J Wagner. and J. Liu. 1996. Washing and Causfic Leaching of Rantoro Tank Situdges: Results af FY 1996 Studres,

PNNt-11278, Rev. 1, Pacific Northwest Natıonal Laboratory. Richland, Washington 
BY.110 TTEPOO, EB]

\begin{tabular}{|c|c|c|c|c|c|c|c|c|c|}
\hline 1 & $\begin{array}{l}\text { Density (g/mL) } \\
\text { volume (kL) }\end{array}$ & & & & & & & & \\
\hline & summation & & & & Wa & & & Leach & \\
\hline Component & $\begin{array}{r}\text { ug'g sample } \\
\text { [Lumetta 1996) }\end{array}$ & $\begin{array}{r}\% \\
\text { recovery }\end{array}$ & $\begin{array}{l}\text { uglg sample } \\
\text { [Appendix) }\end{array}$ & $\begin{array}{r}M T \\
{[\operatorname{tank})}\end{array}$ & $\begin{array}{l}\text { \% remaved } \\
\text { initial solids }\end{array}$ & MT removed & $\begin{array}{r}\text { \% removed } \\
\text { washed solids }\end{array}$ & MT removed & $\begin{array}{r}\% \text { leach } \\
\text { initial solids }\end{array}$ \\
\hline$\overline{A g}$ & & & 14.4 & 0.03 & & 0.000 & 0 & 0.000 & \\
\hline $\mathrm{Al}$ & 36000 & 130 & 13200 & 29.83 & 94 & 28.038 & 33 & 0.597 & 2 \\
\hline As & & & & 0.00 & & 0.000 & 0 & 0.000 & \\
\hline$B$ & & & 79.9 & 0.18 & 100 & 0.181 & \#DIVIO! & \#DIVIO! & 0 \\
\hline $\mathrm{Ba}$ & 108 & 132 & 58.8 & 0.13 & 0 & 0.000 & 0 & 0.000 & 0 \\
\hline $\mathrm{Be}$ & & & & 0,00 & & 0.000 & 0 & 0.000 & \\
\hline $\mathrm{Bi}$ & 861 & & 939 & 2.10 & 0 & 0.000 & 0 & 0.000 & 0 \\
\hline $\mathrm{Ca}$ & 10100 & 109 & 1110 & 2.51 & 0 & 0.000 & 0 & 0.000 & 0 \\
\hline $\mathrm{Cd}$ & 16.8 & & 22.1 & 0.05 & 0 & 0.000 & 0 & 0.000 & 0 \\
\hline $\mathrm{Ce}$ & & & & 0.00 & & 0.000 & 0 & 0.000 & \\
\hline$c_{0}$ & & & 26.6 & 0.06 & & 0.000 & 0 & 0.000 & \\
\hline $\mathrm{Cr}$ & 4160 & 137 & 2780 & 6.28 & 47 & 2952 & 2 & 0.063 & 1 \\
\hline $\mathrm{cu}$ & & & 12.2 & 0.03 & & 0.000 & 0 & 0,000 & \\
\hline $\mathrm{Fe}$ & 13900 & & 961 & 2.17 & 3 & 0.065 & 0 & 0.000 & 0 \\
\hline k & & & 1820 & 4.11 & 69 & 2.838 & 32 & 0.411 & to \\
\hline La & & & & 000 & & 0.000 & 0 & 0.000 & \\
\hline $\mathrm{Li}$ & & & & 0.00 & & 0.000 & 0 & 0.000 & \\
\hline $\mathrm{Mg}$ & 664 & & 120 & 0.27 & & 0.000 & 0 & 0000 & 0 \\
\hline $\mathrm{Mn}$ & 209 & 46 & 48.8 & 0.11 & & 0.000 & 0 & 0.000 & 0 \\
\hline Mo & & & & 0.00 & & 0.000 & 0 & 0.000 & \\
\hline $\mathrm{Na}$ & 182000 & 75 & 254000 & 573.95 & 98 & 562.471 & 0 & 0.000 & \\
\hline $\mathrm{Ni}$ & & & 301 & 0.68 & 0 & 0.000 & 0 & 0.000 & \\
\hline $\mathrm{Pb}$ & 560 & & 962 & 2.17 & 14 & 0.304 & 15 & 0.283 & 13 \\
\hline$P$ & & & & 0.00 & & 0.000 & 0 & 0.000 & \\
\hline$R h$ & & & & 0.00 & & 0,000 & 0 & 0.000 & \\
\hline $\mathrm{Ru}$ & & & & 0.00 & & 0.000 & 0 & 0.000 & \\
\hline sb & & & & 0.00 & & 0.000 & 0 & 0.000 & \\
\hline Se & & & & 0.00 & & 0.000 & 0 & 0.000 & \\
\hline$S_{1}$ & 1450 & 40 & 685 & 1.55 & 16 & 0.248 & 56 & 0.727 & 47 \\
\hline Sn & & & & 0.00 & & 0.000 & 0 & 0.000 & \\
\hline St & 4050 & 164 & 58.5 & 0.13 & 0 & 0.000 & 0 & 0.000 & \\
\hline $\mathrm{Te}$ & & & & 0.00 & & 0.000 & 0 & 0.000 & \\
\hline Th & & & & 0.00 & & 0.000 & 0 & 0.000 & \\
\hline $\mathrm{Ti}$ & & & & 0.00 & & 0.000 & 0 & 0.000 & \\
\hline 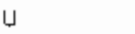 & +5300 & 94 & 4140 & 9.35 & 2 & 0.187 & 0 & 0.000 & 0 \\
\hline v & & & & 0.00 & & 0.000 & 0 & 0.000 & \\
\hline$w$ & & & & 0.00 & & 0000 & 0 & 0.000 & \\
\hline Y & & & & 0.00 & & 0.000 & 0 & 0.000 & \\
\hline $\mathrm{Zn}$ & 67.5 & & 39.9 & 0.09 & 8 & 0.007 & $\mathrm{~s}$ & 0.007 & 8 \\
\hline$z_{r}$ & 27.7 & & 58.5 & 0.13 & 0 & 0000 & 0 & 0.000 & 0 \\
\hline
\end{tabular}

\begin{tabular}{|c|c|c|c|c|c|c|c|c|c|}
\hline \multirow[b]{2}{*}{ Component } & \multirow{2}{*}{$\begin{array}{r}\text { summation } \\
\text { uglg sample } \\
\text { [Lumetta 1996) }\end{array}$} & \multirow[b]{2}{*}{$\begin{array}{r}\% \\
\text { recovery }\end{array}$} & \multirow[b]{2}{*}{$\begin{array}{l}\text { ugly sample } \\
\text { \{Appendix\} }\end{array}$} & \multirow[b]{2}{*}{$\begin{array}{r}M T \\
{[\operatorname{tankk1}]}\end{array}$} & \multicolumn{2}{|c|}{ Wash } & \multicolumn{3}{|c|}{ Leach } \\
\hline & & & & & $\begin{array}{l}\text { \% removed } \\
\text { initial solids }\end{array}$ & MT removed & $\begin{array}{r}\% \text { removed } \\
\text { washed solids }\end{array}$ & MT removed & $\begin{array}{l}\text { \% removed } \\
\text { initial solifts }\end{array}$ \\
\hline $\cos (-2)$ & & & 1910000 & 431.59 & & 0.000 & 0 & 0.000 & \\
\hline NOS- & & & 216000 & 488.09 & & 0.000 & 0 & 0.000 & \\
\hline NO2- & & & 22500 & 50.84 & & 0.000 & 0 & 0.000 & \\
\hline $\mathrm{PO} 4-3$ & 21700 & & 9840 & 22.23 & 19 & 4.225 & 5 & 0.889 & 4 \\
\hline SOA-2 & & & 232000 & 52.42 & & 0.000 & 0 & 0.000 & \\
\hline $\mathrm{F}$ - & & & 6450 & 14.57 & & 0000 & 0 & 0.000 & \\
\hline $\mathrm{Cl}-$ & & & 1640 & 3.71 & & 0.000 & 0 & 0.000 & \\
\hline $\mathrm{Br}$. & & & & 0.00 & & 0.000 & 0 & 0.000 & \\
\hline & $\begin{array}{r}\text { uCilg sample } \\
\text { [Lumetta 1996) }\end{array}$ & $\begin{array}{r}\% \\
\text { recovery }\end{array}$ & $\begin{array}{l}\text { uCUy sample } \\
\text { (Appendix\} }\end{array}$ & $\begin{array}{r}c i \\
\tan k t\end{array}$ & $\begin{array}{l}\text { \% removed } \\
\text { initial sollds }\end{array}$ & Ci removed & $\begin{array}{l}\% \text { removed } \\
\text { washed solids }\end{array}$ & $\mathrm{Cl}$ removed & $\begin{array}{l}\% \text { removed } \\
\text { initial solids }\end{array}$ \\
\hline Total alpha & & & & 0.00 & & 0.000 & 0 & 0.000 & \\
\hline Pu-239, 240 & & & 0071 & 160.43 & & 0.000 & 0 & 0.000 & \\
\hline Am-241+Pu-238 & & & & 0.00 & & 0.000 & 0 & 0.000 & \\
\hline Am-241 (gamma) & & & 0.409 & 924.19 & & 0.000 & 0 & 0.000 & \\
\hline $\mathrm{Cm}-243,244$ & & & & 0.00 & & 0.000 & 0 & 0.000 & \\
\hline C. 137 & & & 579 & 130833.45 & & 0.000 & 0 & 0.000 & \\
\hline Co-60 & & & 0.019 & 42.93 & & 0.000 & 0 & 0.000 & \\
\hline$E u-154$ & & & & 0.00 & & 0.000 & 0 & 0.000 & \\
\hline Eu-155 & & & & 0.00 & & 0.000 & 0 & 0.000 & \\
\hline Sr-90 & & & 58.5 & 13248923 & & 0.000 & 0 & 0.000 & \\
\hline$T_{\varepsilon-9}-9$ & & & & 0.00 & & 0.000 & 0 & 0.000 & \\
\hline
\end{tabular}

Notes:

Nokiceably large concentration of $\mathrm{CO} 3$ (consistent with ion balance).

Analyte concentrations among many segments analyzed by Characterization varied considerably.

Radionuclide data unavaliable.

Lumera, G. J., B. M. Rapko, M J Wagner, and J. Liu. 1996 Washing and Caustic Leaching of Hanford Tank Studges: Results of fy 1996 Studies.

PNNL-11278 Rev. 1. Pactic Northwest National Laboratory, Richland, Washington. 


\begin{tabular}{|c|c|c|c|c|c|c|c|c|c|}
\hline \multicolumn{10}{|c|}{\begin{tabular}{|c|}
1.34 Density $(\mathrm{g} / \mathrm{mL})$ \\
235 Volume $(\mathrm{kL})$
\end{tabular}} \\
\hline \multirow[b]{2}{*}{ Component } & \multirow{2}{*}{$\begin{array}{r}\text { summation } \\
\text { ugig sansple } \\
\text { (Rapko 1995) }\end{array}$} & \multirow[b]{2}{*}{$\begin{array}{r}\% \\
\text { recovery }\end{array}$} & \multirow[b]{2}{*}{$\begin{array}{l}\text { ugig sample } \\
\text { (Colton 1995) }\end{array}$} & \multirow[b]{2}{*}{$\begin{array}{r}M T \\
\{\operatorname{tank}\}\end{array}$} & \multicolumn{2}{|c|}{ Wash } & \multicolumn{3}{|c|}{ Leach } \\
\hline & & & & & $\begin{array}{r}\% \text { removed } \\
\text { initlal solids }\end{array}$ & MT remaved: & $\begin{array}{l}\% \text { removed } \\
\text { washed solids }\end{array}$ & MT removed & $\begin{array}{r}\% \text { leach } \\
\text { initial solids }\end{array}$ \\
\hline $\mathrm{Rg}$ & 369 & 110 & & 0.12 & 0 & 0.000 & 0 & 0.000 & 0 \\
\hline $\mathrm{Al}$ & 6150 & +13 & 14500 & 4.56 & ㅁ & 0.000 & 48 & 2.189 & 48 \\
\hline As & 27.4 & & & 001 & B & 0.000 & 100 & 0.009 & 100 \\
\hline$B$ & 416 & 107 & 24 & 0.00 & 0 & 0.000 & 0 & 0.000 & 0 \\
\hline Ba & 463 & 60 & 4960 & 1.56 & 1 & 0.016 & 0 & 0.000 & 0 \\
\hline $\mathrm{Be}$ & 0.142 & & & 0.00 & 0 & 0.000 & 100 & 0.000 & 100 \\
\hline BI & 2.14 & & 254 & 0.08 & 0 & 0.000 & 100 & 0.080 & 100 \\
\hline $\mathrm{Ca}$ & 2790 & 40 & 11100 & 3.49 & 1 & 0.035 & 0 & 0.000 & o \\
\hline cot & 235 & 68 & 509 & 0.16 & 1 & 0.002 & 0 & 0.000 & 0 \\
\hline $\mathrm{Ce}$ & 169 & 48 & & 0.05 & 0 & 0.000 & 0 & 0.000 & 0 \\
\hline Co & 26.5 & 68 & 63.8 & 0.02 & 0 & 0.000 & 2 & 0.000 & 2 \\
\hline $\mathrm{Cr}$ & 447 & 72 & 619 & 0.15 & 2 & 0.004 & 10 & 0.019 & 10 \\
\hline $\mathrm{Cu}$ & 1620 & 73 & 855 & 0.27 & 7 & 0.019 & 9 & 0.022 & 8 \\
\hline $\mathrm{Fe}$ & 64600 & 83 & 86700 & 27.26 & 0 & 0.000 & 0 & 0.000 & 0 \\
\hline k & & & 1400 & 0.44 & & 0.000 & 0 & 0.000 & \\
\hline La & 200 & 64. & & 0.06 & 0 & 0.000 & 0 & 0.000 & 0 \\
\hline LI & 4.05 & & & 0.00 & 100 & $0.001:$ & HDND! & FDIVIO! & D \\
\hline $\mathrm{Mg}$ & 270 & 69 & 6190 & 1.95 & 3 & 0.058 & 0 & 0.000 & 0 \\
\hline Mn & 1710 & & 2480 & 0.78 & 0 & 0.000 & 0 & 0.000 & 0 \\
\hline Mo & 4.55 & & & 0.00 & 89 & 0.001 & 100 & 0.000 & 11 \\
\hline $\mathrm{Na}$ & & & 50500 & 15.88 & 15 & 2.382 & 0 & 0.000 & \\
\hline $\mathrm{Ni}$ & & & 915 & 0.29 & & 0.000 & 0 & 0.000 & \\
\hline$P b$ & 1990 & 72 & 3560 & 1.12 & 0 & 0.000 & 0 & 0.000 & 0 \\
\hline $\mathrm{Pa}$ & & & & 0.00 & & 0.000 & 0 & 0.000 & \\
\hline Rh & & & & 0.00 & & 0.000 & 0 & 0.000 & \\
\hline Ru & 615 & 79 & & 0.19 & 0 & 0.000 & 0 & 0.000 & 0 \\
\hline$S b$ & & & & 0.00 & & 0.000 & 0 & 0.000 & \\
\hline $\mathrm{Se}$ & 2.52 & & & 0.00 & D & 0.000 & 100 & 0,001 & 100 \\
\hline$S i$ & 10500 & & 70600 & 22.20 & 0 & 0.000 & 1 & 0.222 & 1 \\
\hline Sn & 279 & & & 0.01 & 0 & 0.000 & 100 & 0.009 & $\tan$ \\
\hline Sr & 615 & 79 & 117 & 0.04 & 0 & 0.000 & 0 & 0.000 & 0 \\
\hline $\mathrm{Te}$ & & & & 0.00 & & 0.000 & 0 & 0.000 & \\
\hline Th & & & & 0.00 & & 0.000 & 0 & [1.000 & \\
\hline $\mathrm{Ti}$ & 129 & 81 & & 0.04 & 0 & 0.000 & 0 & 0.000 & 0 \\
\hline u & 2180 & 184 & 3440 & 1.08 & 73 & 0.790 & 7 & 0.022 & 2 \\
\hline$v$ & 1.46 & & & 0.00 & 0 & 0.000 & 100 & 0.000 & 100 \\
\hline W & 21 & & & 0.01 & 36 & 0.003 . & 98 & 0.004 & 61 \\
\hline$Y$ & 615 & 53 & & 0.02 & 0 & 0.000 & 0 & 0.000 & 0 \\
\hline $2 \pi$ & 184 & 68. & 175 & 0.06 & 2 & 0.001 & 16 & 0.009 & 16 \\
\hline$Z_{r}$ & 6690 & 75 & 12200 & 3.84 & 1 & 0.038 & 0 & 0.000 & a \\
\hline
\end{tabular}

\begin{tabular}{|c|c|c|c|c|c|c|c|c|c|}
\hline \multirow[b]{2}{*}{ Component } & \multirow{2}{*}{$\begin{array}{r}\text { summation } \\
\text { uglg sample } \\
\text { (Rapko 1995) }\end{array}$} & \multirow[b]{2}{*}{$\begin{array}{r}\% \\
\text { recovery }\end{array}$} & \multirow[b]{2}{*}{$\begin{array}{l}\text { ug/g sample } \\
\text { (Colton 1995) }\end{array}$} & \multirow[b]{2}{*}{$\begin{array}{r}\text { MT } \\
{[\tan k)}\end{array}$} & \multicolumn{2}{|c|}{ Wash } & \multicolumn{3}{|c|}{ Leach } \\
\hline & & & & & $\begin{array}{l}\text { \% removed } \\
\text { initial solids }\end{array}$ & MT removed & $\begin{array}{r}\% \text { removed } \\
\text { washed solids }\end{array}$ & MT removed & $\begin{array}{l}\text { \% romoved } \\
\text { initial salids }\end{array}$ \\
\hline $\mathrm{OH} \cdot$ & & & & 0.00 & & 0.000 & 0 & 0.000 & \\
\hline NO3- & & & 2810 & 0.88 & & 0.000 & 0 & 0000 & \\
\hline NO2- & & & & 0.00 & & 0.000 & 0 & 0.000 & \\
\hline $\mathrm{PO} 4-3$ & 8180 & 140 & 12500 & 3.93 & 27 & 1.061 & 55 & 1572 & 40 \\
\hline $\mathrm{SO} 4-2$ & & & & 0.00 & & 0.000 & 0 & 0.000 & \\
\hline F- & & & & 0.00 & & 0.000 & 0 & 0.000 & \\
\hline $\mathrm{Cr}$ & & & & 0.00 & & 0.000 & D & 0.000 & \\
\hline Br- & & & & 000 & & 0.000 & 0 & 0.000 & \\
\hline & $\begin{array}{r}\text { summation } \\
\text { uCl/g samplo } \\
\text { \{Rapko 1995\} }\end{array}$ & $\begin{array}{r}\% \\
\text { recovery }\end{array}$ & \begin{tabular}{|l|} 
uClig sample \\
(Colton 1995)
\end{tabular} & $\begin{array}{r}c i \\
{[\tan k]}\end{array}$ & $\begin{array}{l}\% \text { removed } \\
\text { initial solids }\end{array}$ & Ci removed & $\begin{array}{l}\% \text { removed } \\
\text { washed salids }\end{array}$ & Cl removed & $\begin{array}{l}\text { \% remaved } \\
\text { initial solids }\end{array}$ \\
\hline Total alpha & 778 & 72 & & 2446.48 & 0 & 0.000 & 0 & 0.000 & 0 \\
\hline Pu-239, 240 & 1.3 & 69 & 136 & 427663 & $\uparrow$ & 42766 & 0 & 0.000 & \\
\hline Am-241+Pu-238 & 6.38 & 72 & & 2006.24 & 0 & 0.000 & 0 & 0.000 & \\
\hline Am-241 (gamma) & 5.99 & 70 & 1.35 & 424.52 & 0 & 0000 & 0 & 0.000 & 0 \\
\hline $\mathrm{Cm}-243,244$ & & & & 0.00 & & 0.000 & 0 & 0.000 & \\
\hline Cs-137 & 103 & 112 & 594 & 1867879 & 20 & 3735.759 & 30 & 4482.910 & 24 \\
\hline Co-60 & & & 3.95 & 1242.11 & & 0.000 & 0 & 0.000 & \\
\hline Eu-154 & & & & 0.00 & & 0.000 & o & 0.000 & \\
\hline Eu-15s & & & & 0.00 & & 0.000 & 0 & 0.000 & \\
\hline $5 r-90$ & 3040 & 79 & 2710 & 852160.64 & 0 & 0.000 & 0 & 0000 & c \\
\hline Tc-99 & 0.0166 & 203. & 0.264 & 83.02 & 83 & 68.904 & 35 & 4.981 & $\varepsilon$ \\
\hline
\end{tabular}

Notes:

Na measurement impractical due to method of sample prep (Rapko 1995); therefore. Na wash factor based on Colton 1995.

Bi value from Colton 1995 corrected

Aruon measurements could not eastly be determined due to matrix effects (Rapkn 1995): NO3 concentration relatively low, assume wash factor of $100 \%$

Colton, N G. 1995. Studge Pretreatment Chenisty Evaluation: Enhanced Siudge Washing Separation Factors, PNL-10512.

Pacific Northwest National Laboratory, Rıchland Wastington

Rapko, B. M., G. J. Lumetta, M. J. Wagner 1995. Washing and Caustic Leaching of Hantord Tank Situdges. Results of FY1995 Studies, PNL-10712.

Paçific Northwest National Laboratory, Rıchland Washington. 
C-107 (BjPO4 1C, CW) LANL Experimental Resuits

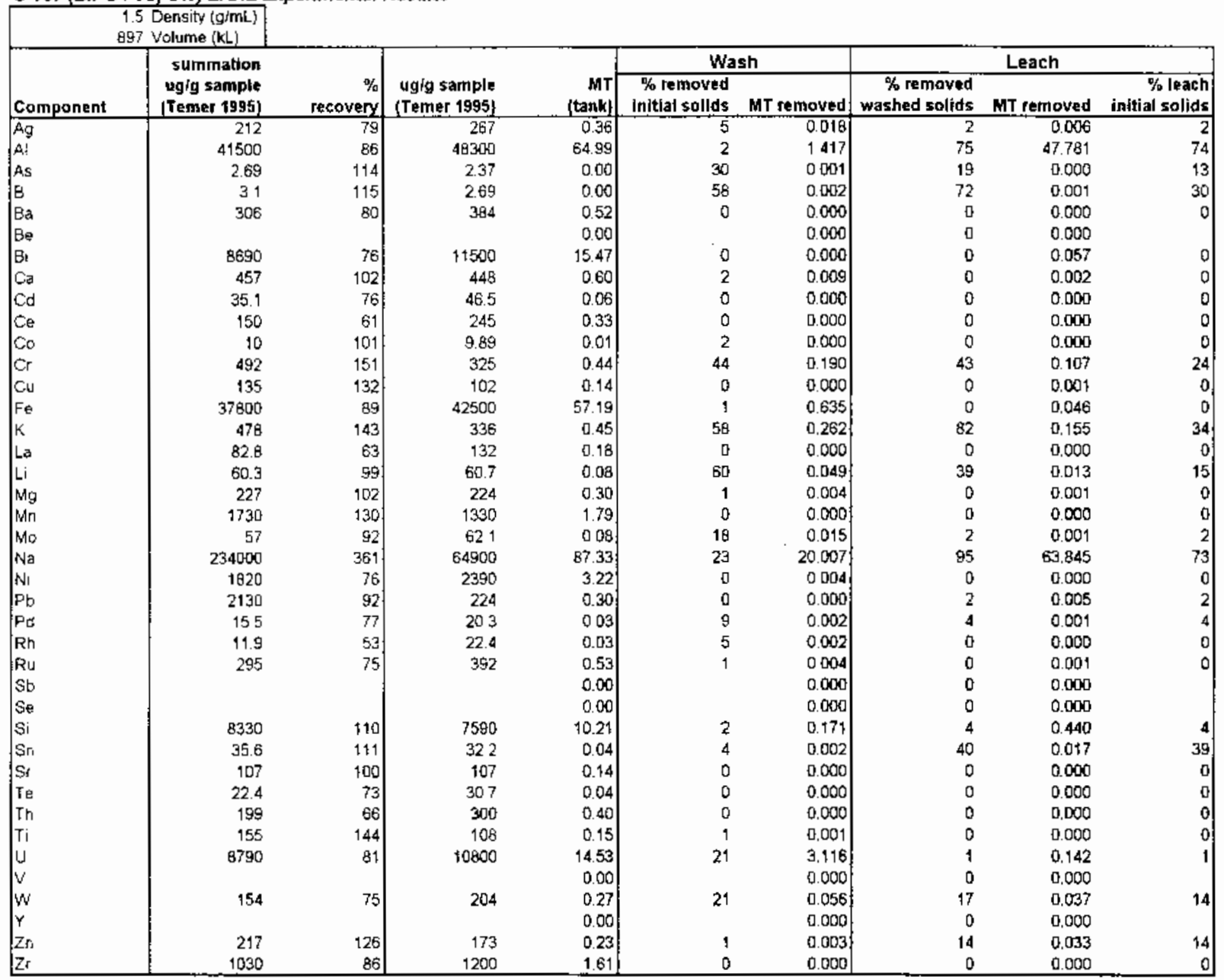

\begin{tabular}{|c|c|c|c|c|c|c|c|c|c|}
\hline \multirow[b]{2}{*}{ Camponent } & \multirow{2}{*}{$\begin{array}{l}\text { summation } \\
\text { uglg sample } \\
\text { (Temer 1995) }\end{array}$} & \multirow[b]{2}{*}{$\begin{array}{r}\% \\
\text { recovery }\end{array}$} & \multirow[b]{2}{*}{$\begin{array}{l}\text { uglg sample } \\
\text { (Temer 1995) }\end{array}$} & \multirow[b]{2}{*}{$\begin{array}{r}M T \\
{[\tan k)}\end{array}$} & \multicolumn{2}{|c|}{ Wash } & \multicolumn{3}{|c|}{ Leach } \\
\hline & & & & & $\begin{array}{l}\% \text { removed } \\
\text { initlal solids }\end{array}$ & MT removed & $\begin{array}{l}\% \text { removed } \\
\text { washed sollds }\end{array}$ & MT removed & $\begin{array}{l}\% \text { removed } \\
\text { initial solids }\end{array}$ \\
\hline $\mathrm{OH}-$ & & & & 0.00 & & 0.000 & 0 & 0.000 & \\
\hline NO3- & & & & 000 & & 0.000 & 0 & 0000 & \\
\hline $\mathrm{NO}_{2}$ & & & & 0.00 & & 0.000 & 0 & 0.000 & \\
\hline POA-3 & 48300 & & 50400 & 67.82 & 48 & 32.321 & 89 & 31.718 & 47 \\
\hline $504-2$ & 6210 & 104 & 5980 & 8.05 & 89 & 7152 & 5 & 0.043 & $i$ \\
\hline F. & 5750 & 128 & 4510 & 6.07 & 75 & 4.570 & 73 & $t .091$ & 18 \\
\hline C1- & & & & 0.00 & & 0.000 & 0 & 0.000 & \\
\hline $\mathrm{Br}$. & & & & 0.00 & & 0.000 & 0 & 0.000 & \\
\hline & $\begin{array}{l}\text { summation } \\
\text { ucilg sample } \\
\text { [Temer 1995] }\end{array}$ & $\begin{array}{r}\% \\
\text { recovery }\end{array}$ & $\begin{array}{l}\text { uCilg sample } \\
\text { [Temer 1995) }\end{array}$ & $\begin{array}{r}c i \\
{[\tan k]}\end{array}$ & $\begin{array}{l}\% \text { removed } \\
\text { initial solids }\end{array}$ & Cl removed & $\begin{array}{l}\% \text { removed } \\
\text { washed solids }\end{array}$ & Ci removed & $\begin{array}{l}\% \text { removed } \\
\text { initlal sollds }\end{array}$ \\
\hline Total atpha & 3.52 & 75 & 4.69 & $6340 . \overline{71}$ & 0 & 0.000 & 0 & 0.000 & $\overline{0}$ \\
\hline Pu-239. 240 & 1.21 & 91 & 1.33 & 1789.60 & B & 0.000 & 0 & 0.000 & 0 \\
\hline Am. $241+$ PUl-238 & & & & 0.00 & & 0.000 & 0 & 0.000 & \\
\hline Am-241 (gamma) & 0.598 & 115 & 0.524 & 705.08 & 0 & 0.000 & 0 & 0000 & 0 \\
\hline $\mathrm{Cm}-243244$ & & & & 0.00 & & 0.000 & 0 & 0.000 & \\
\hline Cs- 137 & 328 & 95 & 34.7 & 46694.19 & 29 & 13769.233 & 62 & 20263.977 & 43 \\
\hline $\mathrm{Co} \cdot 60$ & & & & 0.00 & & 0.000 & 0 & 0,000 & \\
\hline Eu-154 & & & & 0.00 & & 0.000 & 0 & 0.000 & \\
\hline Eu-155 & & & & 0.00 & & 0.000 & 0 & 0.000 & \\
\hline Sr-90 & 2250 & 89 & 2530 & 3404285.78 & b & 0000 & 0 & 0.000 & 0 \\
\hline Tc-gg & & & & 0.00 & & 0.000 & 0 & 0000 & \\
\hline
\end{tabular}

Notes:

Temer, D. J. anc R Vallarreal. 1996 Studge Washing and Atkatthe Leaching Tests on Actuat Hantord Tank Studge: FY 1996 Results, LAUR96-2839. Los Alamos National Laboratory, Los Alamos, New Mexico. 
C-107 (BiPO4 1C, CW) PNNL Expenimental Resujts

\begin{tabular}{|c|c|c|c|c|c|c|c|c|c|}
\hline \multicolumn{2}{|c|}{$\begin{array}{c}1.5 \text { Density }(\mathrm{g} / \mathrm{mL}) \\
897 \text { Volume }\{\mathrm{kL}\} \\
\end{array}$} & \multirow[b]{3}{*}{$\begin{array}{r}\% \\
\text { recovery }\end{array}$} & \multirow[b]{3}{*}{$\begin{array}{l}\text { ug/g sample } \\
\text { (Temer 1995) }\end{array}$} & \multirow[b]{3}{*}{$\begin{array}{r}M T \\
{[\tan k]}\end{array}$} & \multirow{2}{*}{\multicolumn{2}{|c|}{ Wash }} & \multirow{2}{*}{\multicolumn{3}{|c|}{ Leach }} \\
\hline & summation & & & & & & & & \\
\hline Component & $\begin{array}{r}\text { uglo sample } \\
\text { [Lumetta 1996] }\end{array}$ & & & & $\begin{array}{l}\% \text { romoved } \\
\text { initial solids }\end{array}$ & MT removed & $\begin{array}{r}\% \text { removed } \\
\text { washed solids }\end{array}$ & MT removed & $\begin{array}{r}\% \text { leach } \\
\text { initial solids } \\
\end{array}$ \\
\hline $\mathrm{Ag}$ & 278 & 71 & 267 & 0.36 & 0 & 0.000 & 2 & 0.007 & 2 \\
\hline A. & 43600 & 109 & 48300 & 64.99 & 1 & 0.650 & 78 & 50.043 & 77 \\
\hline As & 768 & 90 & 2.37 & 0.00 & 0 & 0.000 & 23 & 0.001 & 23 \\
\hline E & 215 & 11 & 2.69 & 0.00 & 20 & 0.001 & 48 & $\$ .001$ & 38 \\
\hline Ba & 644 & 97. & 384 & 0.52 & 0 & 0.000 & 0 & 0.000 & 0 \\
\hline Be & & & & 0.00 & & 0.000 & 0 & 0.000 & \\
\hline $\mathrm{Bi}$ & 99.8 & 80 & 11500 & 15.47 & 0 & 0.000 & 8 & 1.238 & 8 \\
\hline $\mathrm{Ca}$ & 1020 & 61 & 448 & 0.60 & $\Delta$ & 0.024 & 1 & 0.006 & 1 \\
\hline $\mathrm{Cd}$ & 109 & 91 & 46.5 & 0.06 & 0 & 0.000 & 0 & 0.000 & 0 \\
\hline Ce & 351 & 96 & 245 & 033. & 0 & 0.000 & 11 & 0.036 & 11 \\
\hline $\mathrm{Cc}$ & 38,8 & 92 & 9.89 & 0.01 & 0 & 0.000 & 16 & 0.002 & 16 \\
\hline $\mathrm{Cr}$ & 607 & 106 & 325 & 0.44 & 34 & 0.149 & 23 & 0.066 & 15 \\
\hline $\mathrm{Cu}$ & 207 & 82 & 102 & 014 & 0 & 0.000 & 9 & 0.012 & 9 \\
\hline $\mathrm{Fe}$ & 75900 & 92 & 42500 & 5719 & 0 & 0.000 & 0 & 0.000 & 0 \\
\hline K & & & 336 & 0.45 & & 0.000 & 0 & 0.000 & \\
\hline La & 219 & 97 & 132 & 0.18 & 0 & 0000 & 0 & 0.000 & 0 \\
\hline Li & 169 & & 607 & 0.08 & 84 & 0.069 & 31 & 0.004 & 5. \\
\hline $\mathrm{Mg}$ & 366 & 102 & 224 & 030 & 0 & 0.000 & 0 & 0.000 & 0 \\
\hline$M n$ & 4020 & 93 & 1330 & 179 & 0 & 0000 & 0 & 0.000 & a \\
\hline Mo & 52 & 100 & 62.1 & 0.08 & 23 & 0.019 & 皇 & 0.005 & 6) \\
\hline $\mathrm{Na}$ & & & 64900 & 87.33 & & 0.000 & 0 & 0.000 & \\
\hline$N_{i}$ & & & 2390 & 3.22 & & 0000 & 0 & 0.000 & \\
\hline $\mathrm{Po}$ & 4780 & 98 & 224 & 0.30 & 0 & 0.000 & 18 & 0.054 & 18 \\
\hline $\mathrm{Pd}$ & & & 20.3 & 0.03 & & 0000 & 0 & 0000 & \\
\hline Rh & & & 22.4 & 0.03 & & 0.000 & 0 & 0.000 & \\
\hline Ru & & & 392 & 0.53 & & 0.000 & 0 & 0000 & \\
\hline $\mathrm{Sb}$ & & & & 0.00 & & 0.000 & 0 & 0.000 & \\
\hline Se & & & & 0.00 & & 0.000 & 0 & 0.000 & \\
\hline SI & 11300 & 95. & 7590 & 10.21 . & 1 & 0.102 & 24 & 2.451 & 24 \\
\hline $\mathrm{Sn}$ & & & 32.2 & 0.04 & & 0.000 & 0 & 0.000 & \\
\hline $\mathrm{sr}$ & 104 & 94. & 107 & 0.14 & 0 & 0.000 & 0 & 0.000 & 0 \\
\hline Te & & & 30.7 & 004 & & 0.000 & 0 & 0.000 & \\
\hline$T r$ & & & 300 & 0.40 & & 0.000 & 0 & 0.000 & \\
\hline$T_{1}$ & 258 & 94. & 108 & 0.15 & 0 & 0.000 & 0 & 0.000 & 0 \\
\hline แ & 5470 & 107 & 10800 & 14.53 & 5 & 0727 & 18 & 2.470 & 17 \\
\hline V & & & & 0.00 & & 0.000 & 0 & 0.000 & \\
\hline$W$ & & & 204 & 0.27 & & 0.0000 & 0 & 0.000 & \\
\hline$Y$ & & & & 0 叫 & & 0.000 & 0 & 0.000 & \\
\hline$Z n$ & 114 & 68 & 173 & 0.23 & 0 & 0.000 & 26 & 0.061 & 26 \\
\hline $\mathrm{Zr}$ & 4210 & 161 & 1200 & 1.61 & 0 & 0000 & 0 & 0.000 & 0 \\
\hline
\end{tabular}

\begin{tabular}{|c|c|c|c|c|c|c|c|c|c|}
\hline \multirow[b]{2}{*}{ Component } & \multirow{2}{*}{$\begin{array}{r}\text { summation } \\
\text { ugjg sample } \\
\text { (Lumetta 1996) }\end{array}$} & \multirow[b]{2}{*}{$\begin{array}{r}\% \\
\text { recovery }\end{array}$} & \multirow[b]{2}{*}{$\begin{array}{l}\text { uglg sample } \\
\text { (Temer 1995) }\end{array}$} & \multirow[b]{2}{*}{$\begin{array}{r}M T \\
{[\tan k]}\end{array}$} & \multicolumn{2}{|c|}{ Wash } & \multicolumn{3}{|c|}{ Leach } \\
\hline & & & & & $\begin{array}{l}\text { \% removed } \\
\text { initial soljds }\end{array}$ & MT removed & $\begin{array}{l}\text { \% removed } \\
\text { washed solids }\end{array}$ & MT removed & $\begin{array}{l}\text { \% removed } \\
\text { initial solids }\end{array}$ \\
\hline $\mathrm{OH}-$ & & & & 0.00 & & 0.000 & 0 & 0.000 & \\
\hline NO3. & & & & 0.00 & & 0.000 & 0 & 0.000 & \\
\hline $\mathrm{NO}_{2}-$ & & & & 0.00 & & 0.000 & 0 & 0.000 & \\
\hline$P O 4-3$ & 19700 & 151 & 50400 & 67.82 & 69 & 46.793 & 81 & 16.954 & 25 \\
\hline $504-2$ & & & 5980 & 8.05 & & 0.000 & 0 & 0.000 & \\
\hline F- & & & 4510 & 6.07 & & 0.000 & 0 & 0.000 & \\
\hline $\mathrm{Cl}$ & & & & 0.00 & & 0000 & 0 & 0.000 & \\
\hline $\mathrm{Br}-$ & & & & 000 & & 0.000 & 0 & 0.000 & \\
\hline & $\begin{array}{r}\text { summation } \\
\text { ucifg sample } \\
\text { [Lumetta 1996] }\end{array}$ & $\begin{array}{r}\% \\
\text { recovery }\end{array}$ & $\begin{array}{l}\text { vciig sample } \\
\text { (Temer 1995) }\end{array}$ & $\begin{array}{r}c i \\
{[\tan k]}\end{array}$ & $\begin{array}{l}\% \text { removed } \\
\text { initial solids }\end{array}$ & Ci removed & $\begin{array}{l}\text { \% removed } \\
\text { washed solids }\end{array}$ & Ci removed & $\begin{array}{l}\% \text { removed } \\
\text { initial sollds }\end{array}$ \\
\hline Total alpha & 6.67 & 96 & 4.69 & 6310.71 & 0 & 0.000 & 1 & 63.107 & 1 \\
\hline Pu-239, 240 & $23 t$ & 87 & 1.33 & 1789.60 & 1 & 17.896 & 1 & 17.896 & 1 \\
\hline Am-241+Pu-238 & 419 & 98 & & 0.00 & 0 & 0.000 & 0 & 0.000 & 0 \\
\hline Am-241 (gamma) & & 100 & 0.524 & 705.08 & 0 & 0.000 & 0 & 0.000 & 0 \\
\hline $\mathrm{Cm}-243,244$ & 0.179 & 38 & & 0.00 & 0 & 0.000 & 0 & 0.000 & 0 \\
\hline $\operatorname{cs}-137$ & 57 & 98 & 34.7 & 46691.19 & 13 & 6069.855 & 68 & 27547.803 & 59 \\
\hline Co- 60 & 0.915 & 95 & & 0.00 & 0 & 0,000 & 0 & 0.000 & 0 \\
\hline EU-15A & 5.38 & 114 & & 0.00 & 0 & 0.000 & 0 & 0.000 & 0 \\
\hline Ev-155 & $4.7 B$ & 114 & & 6431.81 & 0 & 0.000 & 0 & 0.000 & 0 \\
\hline $5 r-90$ & 3260 & 127 & 2530 & 3404285.78 & 0 & 0.000 & 0 & 0.000 & 0 \\
\hline$T<\times 99$ & 0029 & 142 & & 0.00 & 97 & 0.000 & 0 & 0.000 & 0 \\
\hline
\end{tabular}

Notes:

Component inventones derlved from Temer 1996 (mass balance 95\% vs PNNL mass balance of 85\%).

Lumetta, G. J., B M Rapko. M. J. Wagner, and J Liu 1996. Washing and Causitc Leaching of Hanford tank Siudges: Results of FV 1996 Sickdes PNNL-11278 Rev 1, Pacific Northwest National Laboratory. Richland, Washington

Femer D. I and R. Vallarreal. 1996. Sludge Washing and Alkaline Leaching Tests on Actual Hanford Tank Studge FY 1996 Resufts, LALRR66-2839, L.os Alamos National Laboratory, Los Alamos. New Mexico

B.13 


\begin{tabular}{|c|c|c|c|c|c|c|c|c|c|}
\hline \multirow[b]{3}{*}{ Component } & \multicolumn{9}{|c|}{$\begin{array}{l}1.3 \text { Density }(\mathrm{g} / \mathrm{mL}\} \\
250 \text { Volume }(\mathrm{kL})\end{array}$} \\
\hline & \multirow{2}{*}{$\begin{array}{r}\text { summation } \\
\text { ugig sample } \\
\text { (Temer 1995) }\end{array}$} & \multirow[b]{2}{*}{$\begin{array}{r}\% \\
\text { recovery }\end{array}$} & \multirow[b]{2}{*}{$\begin{array}{l}\text { ug'g sample } \\
\text { [Appendix] }\end{array}$} & \multirow[b]{2}{*}{$\begin{array}{r}\text { MT } \\
\text { [tank] }\end{array}$} & \multicolumn{2}{|c|}{ Wash } & \multicolumn{3}{|c|}{ Leach } \\
\hline & & & & & $\begin{array}{l}\% \text { removed } \\
\text { initial solids }\end{array}$ & MT removed & $\begin{array}{l}\% \text { removed } \\
\text { washed solids }\end{array}$ & MT removed & $\begin{array}{r}\% \text { leach } \\
\text { initlal solids }\end{array}$ \\
\hline $\mathrm{Ag}$ & & & & 0.00 & & 0.000 & 0 & 0.000 & \\
\hline $\mathrm{Al}$ & 77400 & 119 & 52100 & 16.93 & 3 & 0513 & 93 & 15.327 & 91 \\
\hline AS & 1.99 & 233 & & 0.00 & 45 & 0000 & 71 & 0.000 & 39 \\
\hline $\mathrm{B}$ & & & & 0.00 & & 0.000 & 0 & 0.000 & \\
\hline $\mathrm{Ba}$ & & & & 0.00 & & 0.000 & 0 & 0.000 & \\
\hline $\mathrm{Be}$ & & & & 0.00 & & 0.000 & 0 & 0.000 & \\
\hline$B i$ & & & & 0.00 & & 0.000 & 0 & 0.000 & \\
\hline $\mathrm{Ca}$ & & & 12700 & 4.13 & 0 & 0009 & 0 & 0.000 & 0 \\
\hline $\mathrm{Cd}$ & & & & 0.00 & & 0000 & 0 & 0.000 & \\
\hline Ce & & & & 0.00 & & 0.000 & 0 & 0.000 & \\
\hline Co & & & & 0.00 & & 0.000 & 0 & 0.000 & \\
\hline $\mathrm{Cr}$ & 189 & 78 & NR & 0.06 & 76 & 0.047 & 18 & 0.003 & 4 \\
\hline $\mathrm{Cu}$ & 16.7 & 83 & & 0.00 & 0 & 0.000 & 3 & 0.000 & 3 \\
\hline $\mathrm{Fe}$ & 4900 & 153 & 7160 & 2.33 & 9 & 0.202 & 13 & 0.278 & 12 \\
\hline$k$ & & & & 0.00 & & 0.000 & 0 & 0.000 & \\
\hline La & & & & 0.00 & & 0.000 & 0 & 0.000 & \\
\hline $\mathrm{Li}$ & & & & 0.00 & & 0.000 & 0 & 0.000 & \\
\hline $\mathrm{MHg}$ & 197 & 88 & & 0.06 & 1 & 0.001 & 0 & 0.000 & 0 \\
\hline $\mathrm{Mn}$ & 28.6 & & & 0.01 & 1 & 0.000 & 1 & 0.000 & 1 \\
\hline Mo & & & & 0.00 & & 0.000 & 2 & 0.000 & 2 \\
\hline $\mathrm{Na}$ & 75700 & 95. & 94100 & 30.58 & 62 & 18.961 & 0 & 0.000 & \\
\hline $\mathrm{NI}$ & 9720 & 128 & & 3.16 & 0 & 0.015 & 0 & 0.001 & 0 \\
\hline $\mathrm{Pb}$ & 196 & 121. & & 0,00 & 0 & 0.000 & 3 & 0.000 & 3 \\
\hline Pd & & & & 0.00 & & 0.000 & 0 & 0.000 & \\
\hline Rh & 1.5 & 136 . & & 0.00 & 92 & 0.000 & 34 & 0.000 & 3 \\
\hline Ru & 529 & 113 & & 0.00 & 82 & 0.000 & 37 & Q.000 & 7 \\
\hline $\mathrm{Sb}$ & & & & 0.00 & & 0.000 & 0 & 0.000 & \\
\hline $\mathrm{Se}$ & & & & 0.00 & & 0000 & 0 & 0.000 & \\
\hline$S i$ & 1970 & 110. & & 0.62 & 3 & 0.016 & 12 & 0.072 & +2 \\
\hline Sn & 10.1 & 122 & & 0.00 & 44 & 0.001 & 0 & 0.000 & \\
\hline $\mathrm{Sr}$ & 195 & 134. & & 0.06 & 0 & 0.000 & 0 & 0.000 & 0 \\
\hline $\mathrm{Te}$ & & & & 0.00 & & 0.000 & 0 & 0.000 & \\
\hline Th & & & & 000 & & 0.000 & 0 & 0.000 & \\
\hline $\mathrm{T}_{1}$ & 54.2 & 94. & & 000 & 3 & 0000 & 0 & 0.000 & 0 \\
\hline$\cup$ & 2790 & 121. & 421 & 014 & 10 & 0014 & 0 & 0.000 & \\
\hline v & 13.9 & 501 & & 0.00 & 20 & 0000 & 72 & 0.000 & 58 \\
\hline$w$ & 1.44 & 89 & & 0.00 & 56 & 0.000 & 10 & 0.000 & 4 \\
\hline Y & & & & 0.00 & & 0.000 & 0 & 0.000 & \\
\hline$Z n$ & & & & 0.00 & 0 & 0.000 & 0 & 0.000 & \\
\hline $\mathrm{Zr}$ & & & & 000 & 0 & 0.000 & 0 & 0.000 & \\
\hline
\end{tabular}

\begin{tabular}{|c|c|c|c|c|c|c|c|c|c|}
\hline \multirow[b]{2}{*}{ Component } & \multirow{2}{*}{$\begin{array}{r}\text { summation } \\
\text { ugig sample } \\
\text { (Temer 1995) }\end{array}$} & \multirow[b]{2}{*}{$\begin{array}{r}\% \\
\text { recovery }\end{array}$} & \multirow[b]{2}{*}{$\begin{array}{l}\text { ugig sample } \\
\text { Appendix }\end{array}$} & \multirow[b]{2}{*}{$\underset{\text { tank }}{\text { MT }}$} & \multicolumn{2}{|c|}{ Wash } & \multicolumn{3}{|c|}{ Leach } \\
\hline & & & & & $\begin{array}{l}\% \text { removed } \\
\text { initial solids }\end{array}$ & MT removed & $\begin{array}{l}\% \text { romoved } \\
\text { washed solids }\end{array}$ & MT removed & $\begin{array}{l}\% \text { removed } \\
\text { initial solids }\end{array}$ \\
\hline $\mathrm{OH}-$ & & & & 0.00 & & 0.000 & 0 & 0.000 & \\
\hline $\mathrm{NO3}-$ & & & 43400 & 14.11 & & 0.000 & 0 & 0.000 & \\
\hline $\mathrm{NO} 2$ & & & 24100 & 783 & & 0.000 & 0 & 0.000 & \\
\hline $\mathrm{PO} 4-3$ & 76800 & 100 & 77700 & 25.25 & 75 & 18939 & 0 & 0.013 & 0 \\
\hline $\mathrm{SO} 4-2$ & 5590 & 94 & 6690 & 217 & 94 & 2.044 & 7 & 0.009 & 0 \\
\hline $\mathrm{F}-$ & 2030 & 92 & 3740 & 1.22 & 96 & 1167 & 15 & 0.007 & 1 \\
\hline $\mathrm{Cl}$. & & & 729 & 0.24 & & 0.000 & 0 & 0.000 & \\
\hline \multirow[t]{2}{*}{$\mathrm{Br}-$} & & & & 0.00 & & 0000 & 0 & 0.000 & \\
\hline & $\begin{array}{l}\text { summation } \\
\text { ucijg sample } \\
\text { (Termer 1995) }\end{array}$ & $\begin{array}{r}\% \\
\text { recovery }\end{array}$ & $\begin{array}{r}\text { uCifg sample } \\
\text { Appendix }\end{array}$ & $\begin{array}{r}c i \\
\langle\tan k\rangle\end{array}$ & $\begin{array}{l}\% \text { removad } \\
\text { initial solids }\end{array}$ & Ci removed & $\begin{array}{l}\text { \% removed } \\
\text { washed sollds }\end{array}$ & Ci removed & $\begin{array}{l}\% \text { removed } \\
\text { Inltial rolids }\end{array}$ \\
\hline Total alpha & 0.275 & 1d. & & 89.38 & 0 & 0.000 & 0 & 0.000 & 0 \\
\hline Pu-239, 240 & 0.0903 & 9 & 0.006 & 1.95 & 0 & 0.000 & 0 & 0.000 & 0 \\
\hline Am-241+Pu-238 & & & & 0.00 & & 0.000 & 0 & 0.000 & \\
\hline Am-241 (gamma) & & & 0.029 & 9.43 & 0 & 0.000 & 0 & 0.000 & 0 \\
\hline $\mathrm{Cm}-243,244$ & & & & 0.00 & & 0.000 & 0 & 0.000 & \\
\hline Cs -137 & 250.69 & 95 & 118 & 38350.00 & 4 & 1534.000 & 100 & 36789.155 & 90 \\
\hline $\mathrm{Co}-6 \mathrm{C}$ & & & 0.005 & 1.63 & & 0.000 & 0 & 0.000 & \\
\hline$E u-154$ & & & & 0.00 & & 0.000 & 0 & 0.000 & \\
\hline$E u-\uparrow 55$ & & & & 0.00 & & 0.000 & 0 & 0.000 & \\
\hline Sr-90 & 24.7 & 143 & & 8027.50 & 0 & 0.000 & 0 & 0.000 & 0 \\
\hline Tc-99 & & & & 0.00 & & 0.000 & 0 & 0.000 & \\
\hline
\end{tabular}

Notes:

Characterkzaton data worksheet in Appendix

Temer D J. and R Viltarreal 1995. Sfudge Washing and Alkatine Leaching Tests on Actual Hanforo Tank Studge: A Status Report, LAUR 95-2070, Los Alamos Natıonal Laboratory. Los Alamos. New Mexico. 


\begin{tabular}{|c|c|c|c|c|c|c|c|c|c|}
\hline \multirow[b]{3}{*}{ Component } & \multicolumn{9}{|c|}{$\begin{array}{l}1.2 \text { Density }(\mathrm{g} / \mathrm{mL}) \\
235 \text { Volume }(\mathrm{kL})\end{array}$} \\
\hline & \multirow{2}{*}{$\begin{array}{r}\text { range } \\
\text { uglg sampli } \\
\text { [Lumetta 1994] }\end{array}$} & \multirow[b]{2}{*}{$\begin{array}{r}\% \\
\text { recovery }\end{array}$} & \multirow[b]{2}{*}{$\begin{array}{r}\text { uglg sample } \\
\text { (Colton 1995) }\end{array}$} & \multirow[b]{2}{*}{$\begin{array}{r}M T \\
{[\operatorname{tank}]}\end{array}$} & \multicolumn{2}{|c|}{ Wash } & \multicolumn{3}{|c|}{ Leach } \\
\hline & & & & & $\begin{array}{l}\text { \% removed } \\
\text { initial solids }\end{array}$ & MT removed & $\begin{array}{r}\% \text { removed } \\
\text { washed solids }\end{array}$ & MT removed & $\begin{array}{r}\text { \% leach } \\
\text { initial solids }\end{array}$ \\
\hline $\mathrm{Ag}$ & & & & 0.00 & & 0.000 & 0 & 0.000 & \\
\hline $\mathrm{Al}$ & $54000-140000$ & & 122000 & 34.36 & 5 & 1718 & 80 & 26.10 & 76 \\
\hline As & $12 \cdot 510$ & & & 0.00 & & 0.000 & 0 & 0.000 & \\
\hline$B$ & $14-350$ & & 91 & 0.03 & 0 & 0.000 & 0 & 0.000 & \\
\hline Ba & $120-250$ & & 64.3 & 0.02 & 0 & 0.000 & 0 & 0.000 & 0 \\
\hline Be & & & & 0.00 & 0 & 0.000 & 0 & 0.000 & \\
\hline $\mathrm{Bi}$ & $1900-4600$ & & 12300 & 3.46 & & 0.000 & 0 & 0.000 & 0 \\
\hline $\mathrm{Ca}$ & $8000-13000$ & & 19800 & 5.58 & 1 & 0.056 & 1 & 0.056 & 1 \\
\hline $\mathrm{Cd}$ & & & 10 & 0.00 & & 0.000 & 0 & 0.000 & \\
\hline $\mathrm{Ce}$ & 96.540 & & & 0.00 & 0 & 0.000 & 0 & 0.000 & \\
\hline$C_{0}$ & & & & 0.00 & & 0.000 & 0 & 0000 & \\
\hline $\mathrm{Cr}$ & $340-450$ & & 245 & 0.07 & 73 & 0.050 & 52 & 0.010 & 14 \\
\hline $\mathrm{Cu}$ & & & 71 & 0.02 & & 0.000 & 0 & 0.000 & \\
\hline $\mathrm{Fe}$ & $9100 \cdot 16000$ & & 18900 & 5.32 & 4 & 0.213 & 1 & 0.053 & 1 \\
\hline k & & & 597 & 015. & & 0.000 & 0 & 0.000 & \\
\hline La & $770-1300$ & & & 0.00 & ए & 0.000 & 0 & 0.000 & 0 \\
\hline $\mathrm{Li}$ & & & & 0.00 & & 0.000 & 0 & 0.000 & \\
\hline $\mathrm{Mg}$ & 240.970 & & 491 & 0.14 & 0 & 0.000 & 0 & 0.000 & 0 \\
\hline $\mathrm{Mn}$ & $810 \cdot 1400$ & & 123 & 003 & 0 & 0.000 & 0 & 0.000 & 0 \\
\hline Mo & & & & 000 & & 0.000 & 0 & 0.000 & \\
\hline $\mathrm{Na}$ & 57000 & & 84200 & 2371 & 76 & 18.020 & 0 & 0.000 & \\
\hline $\mathrm{Ni}$ & & & 13300 & 375 & & 0.000 & 0 & 0.000 & \\
\hline $\mathrm{Pb}$ & $3400-5000$ & & 4740 & 1.33 & 0 & 0.000 & 43 & 0.574 & 43 \\
\hline Pd & & & & 0.00 & & 0.000 & 0 & 0.000 & \\
\hline $\mathrm{Rh}$ & & & & 0.00 & & 0.000 & a & 0.000 & \\
\hline Ru & & & & 0.00 & & 0.000 & a & 0.000 & \\
\hline St & & & & 0.00 & & 0.000 & 0 & 0.000 & \\
\hline $\mathrm{Se}$ & $16-460$ & & & 0.00 & 0 & 0.000 & 0 & 0.000 & \\
\hline$s_{1}$ & $5400-49000$ & & 9040 & 2.55 & 3 & 0.076 & 13 & 0.331 & 13 \\
\hline Sn & & & & 0.00 & & 0000 & 0 & 0.000 & \\
\hline Sr & $140 \cdot 290$ & & 186 & 0.05 & 0 & 0.000 & 0 & 0.000 & 0 \\
\hline $\mathrm{Te}$ & & & & 0.00 & & 0.000 & 0 & 0.000 & \\
\hline Th & & & & 0.00 & & 0.000 & 0 & 0.000 & \\
\hline$i_{1}$ & & & & 0.00 & & 0.000 & 0 & 0.000 & \\
\hline u & $7300-190000$ & & 8490 & 2.39 & 0 & 0.000 & 0 & 0.000 & 0 \\
\hline$v$ & & & & 0.00 & & 0.000 & 0 & 0,000 & \\
\hline$w$ & & & & 0.00 & & 0.000 & 0 & 0000 & \\
\hline 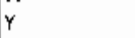 & & & & 0.00 & & 0.000 & 0 & 0.000 & \\
\hline$z_{n}$ & $62-180$ & & 369 & 0.10 & 0 & 0.000 & 0 & 0.000 & 0 \\
\hline $\mathrm{Zr}$ & $120-400$ & & & 0,00 & D & 0.000 & 0 & 0.000 & 0 \\
\hline
\end{tabular}

\begin{tabular}{|c|c|c|c|c|c|c|c|c|c|}
\hline \multirow[b]{2}{*}{ Component } & \multirow{2}{*}{$\begin{array}{r}\text { range } \\
\text { ug/g sample } \\
\text { (Lumetta 1994) }\end{array}$} & \multirow[b]{2}{*}{$\begin{array}{r}\% \\
\text { recovery }\end{array}$} & \multirow[b]{2}{*}{$\begin{array}{l}\text { uglg sample } \\
\text { (Colton 1995) }\end{array}$} & \multirow[b]{2}{*}{$\begin{array}{r}M T \\
(\operatorname{tank})\end{array}$} & \multicolumn{2}{|c|}{ Wash } & \multicolumn{3}{|c|}{ Leach } \\
\hline & & & & & $\begin{array}{l}\% \text { removed } \\
\text { initial solids }\end{array}$ & MT removed & $\begin{array}{r}\% \text { removed } \\
\text { washed sollds }\end{array}$ & MT removed & $\begin{array}{l}\% \text { removid } \\
\text { initial solids }\end{array}$ \\
\hline OF- & & & & 0.00 & & 0.000 & 0 & 0.000 & \\
\hline NO3- & & & 36500 & 10.28 & & 0.000 & 0 & 0.000 & \\
\hline $\mathrm{NO2}-$ & & & 39000 & 10.98 & & 0.000 & 0 & 0.000 & \\
\hline PO4-3 & $22200-288000$ & & 58600 & 16.50 & 30 & 4.951 & 17 & 1.980 & 12 \\
\hline $\mathrm{SO} 4-2$ & & & 6950 & 1.96 & & 0.000 & 0 & 0.000 & \\
\hline F- & & & 400 & 0.11 & & 0.000 & 0 & 0.000 & \\
\hline $\mathrm{Cl}-$ & & & 700 & 0.20 & & 0.000 & 0 & 0.000 & \\
\hline Br. & & & & 0.00 & & 0.000 & 0 & 0.000 & \\
\hline & $\begin{array}{r}\text { range } \\
\text { uCilg sample } \\
\text { fLumetta 1994) }\end{array}$ & $\begin{array}{r}\% \\
\text { recovery }\end{array}$ & $\begin{array}{l}\text { WCify sample } \\
\text { (Colton }\{995 \text { ) }\end{array}$ & $\begin{array}{r}c i \\
\text { (ttank) }\end{array}$ & $\begin{array}{l}\% \text { removed } \\
\text { initial solids }\end{array}$ & Ci remaved & $\begin{array}{r}\% \text { removed } \\
\text { washed solids }\end{array}$ & Ci removed & $\begin{array}{l}\text { \% removod } \\
\text { initial solids }\end{array}$ \\
\hline Total alpha & & & & 0.00 & & 0.000 & $\overline{0}$ & 0.000 & \\
\hline $\mathrm{Pu}-239,240$ & & & 0448 & 126.16 & & 0.000 & 0 & 0.000 & \\
\hline $\mathrm{A} m-241+\mathrm{P}_{\mathrm{u}-238}$ & & & & 0.00 & & 0.000 & 0 & 0.000 & \\
\hline Am-241 (gamma) & & & 0.225 & 6.3. 36 & & 0.000 & 0 & 0.000 & \\
\hline $\mathrm{Cm}-243.244$ & & & & 0.00 & & 0.000 & 0 & 0.000 & \\
\hline C5-137 & $405 \cdot 4170$ & & 715 & 201346.86 & 5 & 10067.343 & 98 & 187252.580 & 93 \\
\hline $\mathrm{Co}-60$ & & & 0.014 & 3.94 & & 0.000 & 0 & 0.000 & \\
\hline Eu-154 & & & & 0.00 & & 0.000 & 0 & 0.000 & \\
\hline$E u-155$ & & & & 0.00 & & 0.000 & a & 0.000 & \\
\hline Sr-90 & $610 \cdot 1640$ & & 1055 & 297092.22 & 0 & 0.000 & 1 & 2970.922 & 1 \\
\hline Tc-99 & $0.04 \cdot 0.044$ & & 0.101 & 28.44 & 95 & 27.020 & 40 & 0.569 & 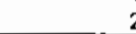 \\
\hline
\end{tabular}

Notes:

Less than wash, leach values entered above as zero

Calton N. G 1995. Sudge Pretreatment Chemisty Evaluation: Enhanced Sludge Woshing Separation Factors. PNL-10512,

Pacific Nonthwest National Laboratory, Richland Washıngton.

Lumera. G J. and B. M. Rapko. 1994 Washing and Atkatine Leaching of Hanford Sludges: A Status Report, PNL-10078.

Pacific Northwest Natıonal Laboratory, Richland Washington.

B. 15 


\begin{tabular}{|c|c|c|c|c|c|c|c|c|c|}
\hline \multicolumn{2}{|c|}{\begin{tabular}{|c|}
1.5 Density $(9 / \mathrm{mL})$ \\
394 Volume $(\mathrm{kL})$
\end{tabular}} & \multicolumn{8}{|l|}{. } \\
\hline \multirow[b]{2}{*}{ Component } & \multirow{2}{*}{$\begin{array}{r}\text { range } \\
\text { ugig sample } \\
\text { (Lumetta 1994) }\end{array}$} & \multirow[b]{2}{*}{ recovery } & \multirow[b]{2}{*}{$\begin{array}{r}\text { ugly sample } \\
\text { \{Colton 1995\} }\end{array}$} & \multirow[b]{2}{*}{$\begin{array}{r}M T \\
\operatorname{tank}\end{array}$} & \multicolumn{2}{|c|}{ Wash } & \multicolumn{3}{|c|}{ Leach } \\
\hline & & & & & $\begin{array}{r}\text { \% removed } \\
\text { initial solids }\end{array}$ & MT removed & $\begin{array}{r}\% \text { removed } \\
\text { washed solids }\end{array}$ & MT removed & $\begin{array}{r}\% \text { leach } \\
\text { initial solids }\end{array}$ \\
\hline $\mathrm{Ag}$ & & & & 0.000 & & 0.000 & 0 & 0.000 & \\
\hline Al & $4500-11000$ & & 18100 & 10.69 & 25 & 2.672 & 80 & 6.412 & 60 \\
\hline As & $17 \cdot 2900$ & & & 000 & 0 & 0.000 & 0 & 0000 & \\
\hline$\theta$ & $840 \cdot 2900$ & & 129 & 008 & 0 & 0.000 & 0 & 0.000 & 0 \\
\hline Ba & $36-290$ & & 869 & 005 & ฤ] & 0.000 & 0 & 0.000 & 0 \\
\hline $\mathrm{Be}$ & & & & 0. 마 마 & & 0.000 & 0 & 0.000 & \\
\hline $\mathrm{Bi}$ & $760-12000$ & & 6400 & 3.78 & 0 & a. 000 & 0 & 0.000 & 0 \\
\hline $\mathrm{Ca}$ & $11000-26000$ & & 24700 & 14.58 & 1 & 0.146 & 0 & 0.000 & 0 \\
\hline Cd & & & 5.5 & 0.00 & & 0.1000 & 0 & 0.000 & \\
\hline $\mathrm{Ce}$ & $665-480$ & & & 000 & & 0.000 & 0 & 0.000 & \\
\hline Co & $45-3400$ & & & 000 & & 0.000 & D & 0.000 & \\
\hline $\mathrm{Cr}$ & $270 \cdot 1400$ & & 251 & 015 & 47 & 0.070 & 75 & 0.059 & 40 \\
\hline $\mathrm{Cu}$ & $59-370$ & & 57.3 & 003 & 0 & 0.000 & 0 & 0.000 & \\
\hline $\mathrm{Fe}$ & $18000-68000$ & & 24400 & 14.41 & 2 & $0.28 \mathrm{~B}$ & 4 & 0.576 & 4 \\
\hline k & & & 625 & 0.37 & & 0.000 & 0 & 0.000 & \\
\hline La & $33-240$ & & & 000 & 0 & 0000 & 0 & 0.000 & 0 \\
\hline $\mathrm{Li}$ & & & & 0.00 & & 0.000 & 0 & 0.000 & \\
\hline $\mathrm{Mg}$ & $170 \cdot 5400$ & & 563 & 0.33 & 0 & 0.000 & 0 & 0.000 & 0 \\
\hline $\mathrm{Mn}$ & $160-770$ & & 236 & 0.14 & 0 & 0.000 & 0 & 0.000 & 0 \\
\hline Mo & & & & 0.00 & & 0.000 & 0 & 0.000 & \\
\hline $\mathrm{Na}$ & 160000 & & 118000 & 69.67 & 72 & 50.165 & 46 & 9.058 & 13 \\
\hline $\mathrm{Ni}$ & & & 14200 & 8.38 & & 0.000 & 0 & 0.000 & \\
\hline $\mathrm{Pb}$ & $770-2600$ & & 2190 & 1.29 & 0 & 0.000 & 0 & 0.000 & 0 \\
\hline Pd & & & & 0.00 & & 0.000 & 0 & 0.000 & \\
\hline Rh & & & & 0.00 & & 0.000 & 0 & 0000 & \\
\hline Ru & & & & 0.00 & & 0.000 & 0 & 0000 & \\
\hline So & & & & 0.00 & & 0.000 & 0 & 0.000 & \\
\hline Se & & & & 0.00 & & 0.000 & 0 & 0000 & \\
\hline $\mathrm{Si}$ & $980 \cdot 28000$ & & 2550 & 1.51 & 4 & 0.060 & 14 & 0196 & 13 \\
\hline Sn & & & & 000 & & 0.000 & 0 & 0.000 & \\
\hline Sr & $370-920$ & & 319 & 0.19 & 0 & 0.000 & D & 0.000 & 0 \\
\hline $\mathrm{Te}$ & & & & 0.00 & & 0.000 & 0 & 0.000 & \\
\hline Th & & & & 0.00 & & 0.000 & 0 & 0.000 & \\
\hline TI & & & & 000 & & 0.000 & 0 & 0.000 & \\
\hline 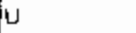 & $99000 \cdot 240000$ & & 59700 & 35.25 & 0 & 0.000 & 0 & 0.000 & 0 \\
\hline v & & & & 0.00 & & 0.000 & 0 & 0.000 & \\
\hline$w$ & & & & 0.00 & & 0.000 & 0 & 0.000 & \\
\hline$Y$ & & & & 0.00 & & 0.000 & 0 & 0.000 & \\
\hline $2 n$ & $170-700$ & & 357 & 0.21 & D & 0.000 & 0 & 0.000 & 0 \\
\hline$Z r$ & $60-1800$ & & 25.7 & 0.02 & 0 & 0.000 & 0 & 0.000 & 0 \\
\hline
\end{tabular}

\begin{tabular}{|c|c|c|c|c|c|c|c|c|c|}
\hline \multirow[b]{2}{*}{ Companent } & \multirow{2}{*}{$\begin{array}{r}\text { range } \\
\text { ug/g sample } \\
\text { (Lumetta t994) }\end{array}$} & \multirow[b]{2}{*}{$\begin{array}{r}\% \\
\text { recovery }\end{array}$} & \multirow[b]{2}{*}{$\begin{array}{r}\text { ugig sample } \\
\text { (Colton 1995) }\end{array}$} & \multirow[b]{2}{*}{$\begin{array}{r}\text { MT } \\
\text { [tank) }\end{array}$} & \multicolumn{2}{|c|}{ Wash } & \multicolumn{3}{|c|}{ Leach } \\
\hline & & & & & $\begin{array}{l}\% \text { remaved } \\
\text { initial soltds }\end{array}$ & MI removed & $\begin{array}{r}\% \text { removed } \\
\text { washed solids }\end{array}$ & MT removed & $\begin{array}{l}\% \text { removed } \\
\text { initial solids }\end{array}$ \\
\hline $\mathrm{OH}-$ & & & & D.00 & & 0.000 & 0 & 0.000 & \\
\hline $\mathrm{NO} 3-$ & & & 75700 & 44.70 & & 0.000 & 0 & 0.000 & \\
\hline NOZ- & & & 57300 & 33.83 & & 0.000 & 0 & 0.000 & \\
\hline PO4-3 & $126000-177000$ & & 88100 & 52.02 & 45 & 23.409 & 71 & 20.288 & 39 \\
\hline$S O 4-2$ & & & 14700 & 8.68 & & 0.000 & 0 & 0.000 & \\
\hline F- & & & 725 & 0.43 & & 0.000 & 0 & 0.000 & \\
\hline Cl- & & & $1+70$ & 0.69 & & 0.000 & 0 & 0.000 & \\
\hline $\mathrm{Br}$. & & & & 0.00 & & 0.000 & 0 & 0.000 & \\
\hline & $\begin{array}{r}\text { range } \\
\text { uCi/g sample } \\
\text { (Lumetta 1994) }\end{array}$ & $\begin{array}{r}\% \\
\text { recovery }\end{array}$ & $\begin{array}{l}\text { uCijg sample } \\
\text { (Colton 1995) }\end{array}$ & $\begin{array}{r}c i \\
\text { (tank) }\end{array}$ & $\begin{array}{l}\% \text { removed } \\
\text { initial solids }\end{array}$ & Ci cembed & $\begin{array}{l}\text { \% removed } \\
\text { washed solids }\end{array}$ & Ci removerd & $\begin{array}{l}\% \text { removed } \\
\text { initial solids }\end{array}$ \\
\hline Total alpha & & & & 000 & & 0.000 & 0 & 0.000 & \\
\hline Fu-239. 240 & & & 0.107 & 63.18 & & 0.000 & 0 & 0.000 & \\
\hline A.m-241+Pu-238 & & & & 000 & & 0.000 & 0 & 0.000 & \\
\hline Am-241 (gamma & & & 0.342 & 20194 & & 0.000 & 0 & 0.000 & \\
\hline Cm-243. 244 & & & & 0.00 & & 0000 & 0 & 0.000 & \\
\hline Cs- 137 & $870-27000$ & & 795 & 46941570 & 4 & 18776.628 & 98 & 441250.758 & 94 \\
\hline Co-60 & & & 0.02 & 11.81 & & 0.000 & to & 0.000 & \\
\hline Eu-154 & & & & 000 & & 0.000 & 0 & 0.000 & \\
\hline Eu-155 & & & & 0.00 & & 0,000 & ㅁ & 0.000 & \\
\hline Sr-90 & $68-250$ & & 2000 & 1180920.00 & 0 & 0000 & 1 & 11809.200 & 1 \\
\hline te-99 & 0.073 & & 0.124 & 7322 & 97 & $7+.021$ & 0 & 0.000 & 0 \\
\hline
\end{tabular}

Notes:

Less than wash. leach values entered above as zero

BI value based on average of values reported in Lumetta 1994; no value reported in characterization report used for Colton 1995.

Colton, N. G. 1995. Studge Pretrealment Chemistry Evaluation: Enhanced Studge Washing Separation Factors, PNL-10512.

Pacific Northwest National Laboralory, Richland Wașington.

Lumetta. G J. and B M Rapko 1994 Washing and Afkatine Leaching of Hanford Siudges: A Status Repart. PNL-10078

Pacific Northwest National Laboratory. Richland Washungton. 


\begin{tabular}{|c|c|c|c|c|c|c|c|c|c|}
\hline \multicolumn{10}{|c|}{$\begin{array}{r}1.5 \text { Density }(\mathrm{g} / \mathrm{mL}) \\
1109 \text { Volume }(\mathrm{kL})\end{array}$} \\
\hline \multirow[b]{2}{*}{ Camponent } & \multirow{2}{*}{$\begin{array}{l}\text { summation } \\
\text { ugig sample } \\
\text { (Terner 1995) }\end{array}$} & \multirow[b]{2}{*}{$\begin{array}{r}\% \\
\text { recovery }\end{array}$} & \multirow[b]{2}{*}{$\begin{array}{r}\text { ugig sample } \\
\text { \{Colton } 1995\} \text {. }\end{array}$} & \multirow[b]{2}{*}{$\begin{array}{r}M T \\
(\tan k)\end{array}$} & \multicolumn{2}{|c|}{ Wash } & \multicolumn{3}{|c|}{ Leach } \\
\hline & & & & & $\begin{array}{c}\% \text { removed } \\
\text { initial solids }\end{array}$ & MT removed & $\begin{array}{r}\% \text { removed } \\
\text { washed solids }\end{array}$ & MT removed & $\begin{array}{r}\% \text { leach } \\
\text { initial solids }\end{array}$ \\
\hline $\mathrm{Ag}$ & & & & 0.00 & & 0.000 & 0 & 0,000 & \\
\hline A.l & 121000 & t11 & 118000 & 196.29 & 4 & 7.263 & 30 & 57.122 & 29 \\
\hline As & & & & 0.00 & & 0.000 & 0 & 0.000 & \\
\hline 8 & & & 392 & 0.07 & 50 & 0.032 & 22 & 0.007 & 11 \\
\hline $\mathrm{Ba}$ & 37.4 & 445 & 33.3 & 0.06 & 0 & 0.000 & 0 & 0.000 & 0 \\
\hline$B e$ & & & & 0.00 & & 0.000 & 0 & 0.000 & \\
\hline BI & 16.8 & 40 & & 0.00 & 0 & 0.000 & 0 & 0.000 & D \\
\hline $\mathrm{Ca}$ & 264 & 130 & 1910 & 318 & 2 & 0.077 & 0 & 0.008 & 0 \\
\hline $\mathrm{Cd}$ & 0.782 & & & 0.001 & 0 & 0.000 & 5 & 0.000 & 5 \\
\hline $\mathrm{Ce}$ & & & & 0.00 & & 0.000 & 0 & 0.000 & \\
\hline Co & & & 673 & 001 & 0 & 0.000 & 0 & 0.000 & 0 \\
\hline $\mathrm{Cr}$ & 2060 & 102 & 4290 & 714 & 87 & 6194 & 73 & 0.684 & 10 \\
\hline $\mathrm{Cu}$ & 50.3 & 107 & 51.8 & 0.09 & 1 & 0.001 & 43 & 0.037 & 42 \\
\hline $\mathrm{Fe}$ & 2530 & 98 & 2490 & 4.14 & 0 & 0.000 & 0 & 0.014 & D \\
\hline$k$ & & & 300 & 0.50 & 1 & 0.004 & 0 & 0.002 & 0 \\
\hline La & 15.9 & & & 0.03 & 0 & 0000 & 0 & 0.000 & 0 \\
\hline $\mathrm{Li}$ & 1.68 & 79 & & 0.0 .0 & & 0.000 & 1 & 0.000 & 1 \\
\hline $\mathrm{Mg}$ & 65.9 & 82 & 53.5 & 0.09 & 0 & 0.000 & 0 & 0.000 & 0 \\
\hline$M n$ & 1110 & 93 & 1090 & 1.81 & 0 & 0000 & 0 & 0.000 & 0 \\
\hline Mo & 15.1 & & & 0.03 & 63 & 0.016 & 24 & 0.002 & 9 \\
\hline $\mathrm{Na}$ & 288000 & 254 & 119000 & 197.96 & 38 & 75481 & 94 & 114.697 & 58 \\
\hline $\mathrm{Ni}$ & & & 56 & 0.09 & 0 & 0000 & 0 & 0.000 & 0 \\
\hline $\mathrm{Pb}$ & 40.1 & 116 & 38.5 & 0.06 & 1 & 0.001 & 24 & 0.015 & 24 \\
\hline Pd & 0.672 & 120 & & 0.00 & 36 & 0.000 & 23 & 0.000 & 15 \\
\hline Rh & 413 & 121 & & 0.01 & 0 & 0.000 & 1 & 0.000 & 1 \\
\hline RL & 13 & 123 & & 0.02 & 0 & 0.000 & 1 & 0.000 & 1 \\
\hline $\mathrm{Sb}$ & & & & 0.00 & & 0.000 & 0 & 0.000 & \\
\hline Se & & & & 0.00 & & 0.000 & 0 & 0.000 & \\
\hline si & $318+$ & 267 & 1460 & 2.43 & 60 & 1.466 & 8 & 0.079 & 3 \\
\hline Sn & 5.92 & 112 & & 0.01 & 40 & 0.004 & 49 & 0.003 & 29 \\
\hline St & 338 & 81. & 418 & 0.70 & 0 & 0.000 & 0 & 0.000 & 0 \\
\hline Te & & & & 0.00 & & 0.000 & 0 & 0.000 & \\
\hline Th & & & & 0,00 & & 0.000 & 0 & 0.000 & \\
\hline $\mathrm{Ti}$ & 135 & 126 & & 0.02 & 1 & 0.000 & 1 & 0.000 & 1 \\
\hline U & 5610 & 131. & 6820 & 11.01 & 0 & 0.000 & 0 & 0.008 & 0 \\
\hline iv & 26.5 & 114 & & 0.04 & 7 & 0.003 & 3 & 0.001 & 3 \\
\hline w & 18.0 & 118 & & 0.03 & 57 & 0.018 & 94 & 0.013 & 41 \\
\hline$Y$ & & & & 0.00 & & 0,000 & 0 & 0.000 & \\
\hline $\mathrm{Zn}$ & 25.4 & 83 & 325 & 0.54 & 26 & 0.138 & 43 & 0.175 & 32 \\
\hline $\mathrm{Zr}$ & & & 645 & 1.07 & 0 & 0.000 & 0 & 0.002 & 0 \\
\hline
\end{tabular}

S.104 (REDOX) LANL Experimental Results (Continued)

\begin{tabular}{|c|c|c|c|c|c|c|c|c|c|}
\hline \multirow[b]{2}{*}{ Component } & \multirow{2}{*}{$\begin{array}{l}\text { summation } \\
\text { ugig sample } \\
\text { [Temer 1995) }\end{array}$} & \multirow[b]{2}{*}{$\begin{array}{r}\% \\
\text { recovery }\end{array}$} & \multirow[b]{2}{*}{$\begin{array}{l}\text { ugig sample } \\
\text { (Colton 1995) }\end{array}$} & \multirow[b]{2}{*}{$\begin{array}{r}M T \\
\text { (tank) }\end{array}$} & \multicolumn{2}{|c|}{ Wash } & \multicolumn{3}{|c|}{ Leach } \\
\hline & & & & & $\begin{array}{l}\% \text { removed } \\
\text { initlal solids }\end{array}$ & MT remayed: & $\begin{array}{r}\% \text { removed } \\
\text { washed solids }\end{array}$ & MT removed & $\begin{array}{l}\% \text { removed } \\
\text { initlal sollds }\end{array}$ \\
\hline $\mathrm{OH}$. & & & & 0.00 & & 0.000 & 0 & 0.000 & \\
\hline $\mathrm{NO}-$ & & & 186000 & 309.41 & & 0.000 & 0 & 0.000 & \\
\hline NO2- & & & 20800 & 34.60 & & 0.000 & 0 & 0.000 & \\
\hline $\mathrm{PO} 4.3$ & 176 & 106 & 289 & 0.48 & 0 & 0.000 & 8 & 0.040 & 8 \\
\hline SO4-2 & 2760 & 87 & 2370 & 3.94 & 47 & 1.846 & 5 & 0.103 & 3 \\
\hline F- & 289 & 68 & 145 & 0.24 & 30 & 0.072 & 9 & 0.015 & 6 \\
\hline Cl- & & & 3170 & 5.27 & & 0.000 & 0 & 0.000 & \\
\hline $\mathrm{Br}-$ & & & & 0.00 & & 0000 & 0 & 0.000 & \\
\hline & $\begin{array}{r}\text { summation } \\
\text { ucifg sample } \\
\text { [Temer 1995] }\end{array}$ & $\begin{array}{r}\% \\
\text { recovery }\end{array}$ & $\begin{array}{l}\text { uCilg sample } \\
\text { (Colton 1995) }\end{array}$ & $\left.\begin{array}{r}c i \\
{[\tan k)}\end{array}\right]$ & $\begin{array}{l}\% \text { removed } \\
\text { initial solids }\end{array}$ & Ci removed & $\begin{array}{l}\% \text { removed } \\
\text { washed solids }\end{array}$ & Ci removed & $\begin{array}{l}\text { \% reinoved } \\
\text { initial solids }\end{array}$ \\
\hline Total alphạ & & & & 0.00 & 0 & 0.000 & 0 & 0.000 & 0 \\
\hline Pu-239, 240 & & & 0.284 & 472.44 & 0 & 0.000 & 0 & 0.000 & 0 \\
\hline$A m-241+P u-238$ & & & & 0,00 & 0 & 0000 & 0 & 0.000 & 0 \\
\hline Am-241 (gamma) & & & 0.118 & 196.29 & & 0.000 & 0 & 0.000 & \\
\hline $\mathrm{Cm}-243.244$ & & & & 000 & & 0.000 & 0 & 0.000 & \\
\hline Cs-137 & & & 63.2 & 105133.67 & 86 & 90551.633 & 71 & 10376.694 & 10 \\
\hline $\mathrm{Co}-60$ & & & 0.033 & 54.90 & & 0000 & 0 & 0.000 & \\
\hline Eu-154 & & & & 0.000 & & 0.000 & 0 & 0.000 & \\
\hline$E_{U}-155$ & & . & & 0.00 & & 0.000 & 0 & 0.000 & \\
\hline Sr-90 & & & 303 & 50404277 & 0 & 0.000 & 0 & 252.021 & 0 \\
\hline Tc-99 & & & 0.025 & 41.59 & 0 & 0.000 & 0 & 0.000 & 0 \\
\hline
\end{tabular}

Golton. N. G. 1995. Sindge Pretreatment Chemistry Evaluation: Enhanced Studge Washing Separalton Factors, PNL-10512,

Pacific Northwest Natıonal Laboratory, Richland Washington.

Temer, D. J. and R. Villarreal. 1995 Siudge Washing and Alkatine Leaching Tests on Actual Hantord Tank Siudge: A Status Reparl, LAUR 95-2070, Los Alamos National Laboratory. Los Alamos, New Mexico 
S-104 (REDOX) PNNL Experimental Results

\begin{tabular}{|c|c|c|c|c|c|c|c|c|c|}
\hline \multicolumn{2}{|c|}{$\begin{array}{r}15 \text { Density }(g / m L) \\
1109 \text { Volume }(\mathrm{kL})\end{array}$} & \multirow[b]{3}{*}{$\begin{array}{r}\% \\
\text { recovery }\end{array}$} & \multirow[b]{3}{*}{$\begin{array}{r}\text { ug/g sample } \\
\text { (Colton 1995) }\end{array}$} & \multirow[b]{3}{*}{$\begin{array}{r}M T \\
{[\operatorname{tank}]}\end{array}$} & \multirow{2}{*}{\multicolumn{2}{|c|}{ Wash }} & \multirow{2}{*}{\multicolumn{3}{|c|}{ Leach }} \\
\hline & summation & & & & & & & & \\
\hline Component & $\begin{array}{l}\text { uglg sample } \\
\text { (Rapko 1995) }\end{array}$ & & & & $\begin{array}{l}\text { \% romoved } \\
\text { initial solids }\end{array}$ & MT remaved & $\begin{array}{l}\% \text { removed } \\
\text { washed solids }\end{array}$ & MT removed & $\begin{array}{r}\% \text { leach } \\
\text { initial solids }\end{array}$ \\
\hline $\mathrm{Ag}$ & 2.15 & & & 0.00 & 0 & 0.000 & 0 & 0.000 & 0 \\
\hline $\mathrm{Al}$ & 115000 & 118 & 118000 & 196.29 & 3 & 5.889 & 36 & 68.703 & 35 \\
\hline As & 153 & 117 & & 0.25 & 0 & 0.000 & 58 & 0148 & 58 \\
\hline$B$ & 216 & 19 & 392 & 0.07 & 0 & 0.000 & 0 & 0.000 & 0 \\
\hline $\mathrm{Ba}$ & 38.7 & 99 & 33.3 & 006 & 0 & 0.000 & 0 & 0,000 & 0 \\
\hline $\mathrm{Be}$ & & & & $0.0 \mathrm{OP}$ & & 0000 & 0 & 0.000 & \\
\hline$B i$ & 17.2 & & & 0.03 & 0 & 0.000 & 0 & 0.000 & 0 \\
\hline $\mathrm{Ca}$ & 610 & 42 & 1910 & 318 & 21 & 0667 & 20 & 0.508 & 16 \\
\hline $\mathrm{Cd}$ & & & & 0.00 & & $0.0 \mathrm{KOO}$ & 0 & 0,000 & \\
\hline $\mathrm{Ce}$ & 19.4 & & & 0.03 & 0 & 0000 & 0 & 0.000 & 0 \\
\hline Co & 4.3 & & 6.73 & 0.01 & 0 & 0.000 & 0 & 0.000 & 0 \\
\hline $\mathrm{Cr}$ & 2670 & 87 & 4290 & 7.14 & 90 & 6.423 & 60 & 0.428 & 6 \\
\hline $\mathrm{Cu}$ & 52.6 & 38 & 51.8 & 0.09 & 0 & 0.000 & 2 & 0.002 & 2 \\
\hline$F_{e}$ & 2610 & 118 & 2490 & 4. 14 & 0 & 0.000 & 0 & 0.000 & 0 \\
\hline$K$ & & & 300 & (3.50) & & 0.000 & 0 & 0.000 & \\
\hline La & 12.9 & & & 0.02 & 0 & 0.000 & 0 & 0.000 & 0 \\
\hline $\mathrm{Li}$ & & & & 0.00 & & 0.000 & 0 & 0.000 & \\
\hline $\mathrm{Mg}$ & 86 & 66 & 535 & 0.09 & 0 & 0.000 & 0 & 0.000 & 0 \\
\hline$M n$ & 1550 & 108 & 1090 & 181 & 0 & 0.000 & 0 & 0.000 & 0 \\
\hline Mo & 0446 & & & 0.00 & 0 & 0.000 & 900 & 0.001 & 100 \\
\hline Na & & & 119000 & 197.96 & 76 & 150448 & 0 & 0,000 & \\
\hline $\mathrm{Ni}$ & & & 56 & 0.09 & & 0.000 & 0 & 0.000 & \\
\hline $\mathrm{Pb}$ & & & 38.5 & 006 & & 0.000 & 0 & 0.000 & \\
\hline $\mathrm{Pd}_{\mathrm{d}}$ & & & & 0.00 & & 0.000 & 0 & 0.000 & \\
\hline$R h$ & & & & 0.00 & & 0.000 & 0 & 0.000 & \\
\hline$R_{u}$ & & & & 0.마 & & 0000 & 0 & 0.000 & \\
\hline $\mathrm{Sb}$ & & & & 0.00 & & 0.000 & 0 & 0.000 & \\
\hline se & 20.4 & & & 003 & 0 & 0.000 & 26 & 0.009 & 26 \\
\hline S. & 1090 & 25 & 1460 & 2.43 & 0 & 0.000 & 0 & 0.000 & 0 \\
\hline Sn & & & & 0.00 & & 0.000 & 0 & 0.000 & \\
\hline$S_{t}$ & 474 & & 418 & 0.70 & 0 & 0.000 & 0 & 0.000 & 0 \\
\hline Te & & & & 0.00 & & प.000 & 0 & 0.000 & \\
\hline Th & & & & 0.00 & & 0.000 & 0 & 0.000 & \\
\hline$T_{1}$ & 14 & & & 0.02 & 0 & 0.000 & 0 & 0.000 & 0 \\
\hline $\mathrm{U}$ & 7630 & 116 & 6620 & $\$ 1.01$ & 0 & 0.000 & 0 & 0.000 & 0 \\
\hline$v$ & & & & 0.00 & & 0.000 & 0 & 0.000 & \\
\hline$W$ & & & & 0.00 & & 0.000 & 0 & 0.000 & \\
\hline$Y$ & 43 & & & 0.01 & 0 & 0.000 & 0 & 0.000 & 0 \\
\hline Zn & 96.5 & 148 & 325 & 0.54 & 54 & 0.292 & 52 & 0.130 & 24 \\
\hline $\mathrm{Zr}$ & 34.4 & & 645 & 1.07 & D & 0.000 & 0 & 0.000 & 0 \\
\hline
\end{tabular}

\begin{tabular}{|c|c|c|c|c|c|c|c|c|c|}
\hline \multirow[b]{2}{*}{ Component } & \multirow{2}{*}{$\begin{array}{r}\text { summation } \\
\text { uglg sample } \\
\text { (Rapko 1995) }\end{array}$} & \multirow[b]{2}{*}{$\begin{array}{r}\% \\
\text { recovery }\end{array}$} & \multirow[b]{2}{*}{$\begin{array}{r}\text { uglg sample } \\
\text { (Colton } 2995 \text { ) }\end{array}$} & \multirow[b]{2}{*}{$\begin{array}{r}M T \\
(\tan k\})\end{array}$} & \multicolumn{2}{|c|}{ Wash } & \multicolumn{3}{|c|}{ Leach } \\
\hline & & & & & $\begin{array}{l}\text { \% removed } \\
\text { initial solids }\end{array}$ & MT removed & $\begin{array}{l}\% \text { removed } \\
\text { washed solids }\end{array}$ & MT removed & $\begin{array}{l}\text { \% remaved } \\
\text { initial solids }\end{array}$ \\
\hline $\mathrm{OH}-$ & & & & 0.00 & & 0000 & 0 & 0.000 & \\
\hline NO3. & 154000 & & 186000 & $309 \mathbf{4 1}$ & 94 & 290.848 & 100 & 18.565 & 6 \\
\hline NO2- & 90600 & & 20800 & 34.50 & 36 & $\{2.456$ & 100 & 22.145 & 64 \\
\hline $\mathrm{PO} 4-3$ & & & 289 & 048 & 45 & 0.216 & 0 & 0.000 & \\
\hline $\mathrm{SO} 4-2$ & 1240 & 9 & 2370 & 3.94 & 100 & 3943 & \#D:1VO! & FDIVNo! & \\
\hline F- & & & 145 & 024 & & 0.000 & 0 & 0.000 & \\
\hline $\mathrm{Cl}$ & 2570 & & 3170 & 5.27 & 91 & 4799 & 89 & 0.422 & 8 \\
\hline Br. & & & & 0.00 & & 0.000 & 0 & 0.000 & \\
\hline & $\begin{array}{r}\text { summation } \\
\text { uCify sample } \\
\text { (Rapko 1995) }\end{array}$ & recovery & $\begin{array}{l}\text { ucilg sample } \\
\text { (Colton 1995) }\end{array}$ & $\begin{array}{r}\mathrm{Ci} \\
(\tan \mathrm{k})\end{array}$ & $\begin{array}{l}\% \text { removed } \\
\text { initial solids }\end{array}$ & Ci removed & $\begin{array}{l}\% \text { removed } \\
\text { washed solids }\end{array}$ & Cl temoved & $\begin{array}{l}\% \text { temoved } \\
\text { initial sollds }\end{array}$ \\
\hline Total alpha & 0584 & 123 & & 971.49 & 0 & 0.000 & 0 & 0.000 & 0 \\
\hline$P_{t u}-239,24 C$ & 0.434 & 122 & 0.284 & 47244 & 0 & 0000 & 0 & 0.000 & 0 \\
\hline$A m-241+P u-238$ & 0151 & 126 & & 000 & 0 & 0.000 & 0 & 0.000 & 0 \\
\hline Am. $2 \Delta 1$ (gamma) & 0.157 & 97 & 0.118 & 19629 & 0 & 0000 & 0 & 0.000 & 0 \\
\hline $\mathrm{Cm}-243.244$ & & & & 0.00 & & 0.000 & 0 & 0,000 & \\
\hline Cs -137 & 57.1 & 96 & 63.2 & 10513367 & 90 & 94620.307 & 80 & 8410.694 & 8 \\
\hline Co-60 & & & 0.033 & 54.9마 & & 0.000 & 0 & 0.000 & \\
\hline Eu-154 & & & & 0.000 & & 0.000 & 0 & 0,000 & \\
\hline Ev- 155 & & & & 0.00 & & 0.000 & 0 & 0.000 & \\
\hline $\mathrm{Sr} .90$ & 351 & 110 & 303 & 50404277 & & 0000 & 0 & 0.000 & 0 \\
\hline Tc-99 & 0.021 & 118 & 0.025 & 4159 & 95 & 39508 & 100 & 2.079 & 5 \\
\hline
\end{tabular}

Na measurement Impractical due to method of sample prep (Rapko 1995); therefore. Na wash factor based on Colton 1995.

PO4 measurement below detection limit for Rapko 1995; therefore PO4 wash factor based on Colton 1995

Colton, N G 1995 Situdge Pretreatment Chemisty Evaluation Enhanced Siudge Wasining Separation Factors, PNL-10512.

Pacific Northwest National Laboratory, Richtand Washington

Rapkc. B M.G J tumetta. M. J. Wagner 1995. Washing and Caustic Lagehtng of Hanhord Tank Sludges Resutts of FY1995 Studies, PNL-10712,

Pacific Northwast National Laboratory. Richland Washangton. 


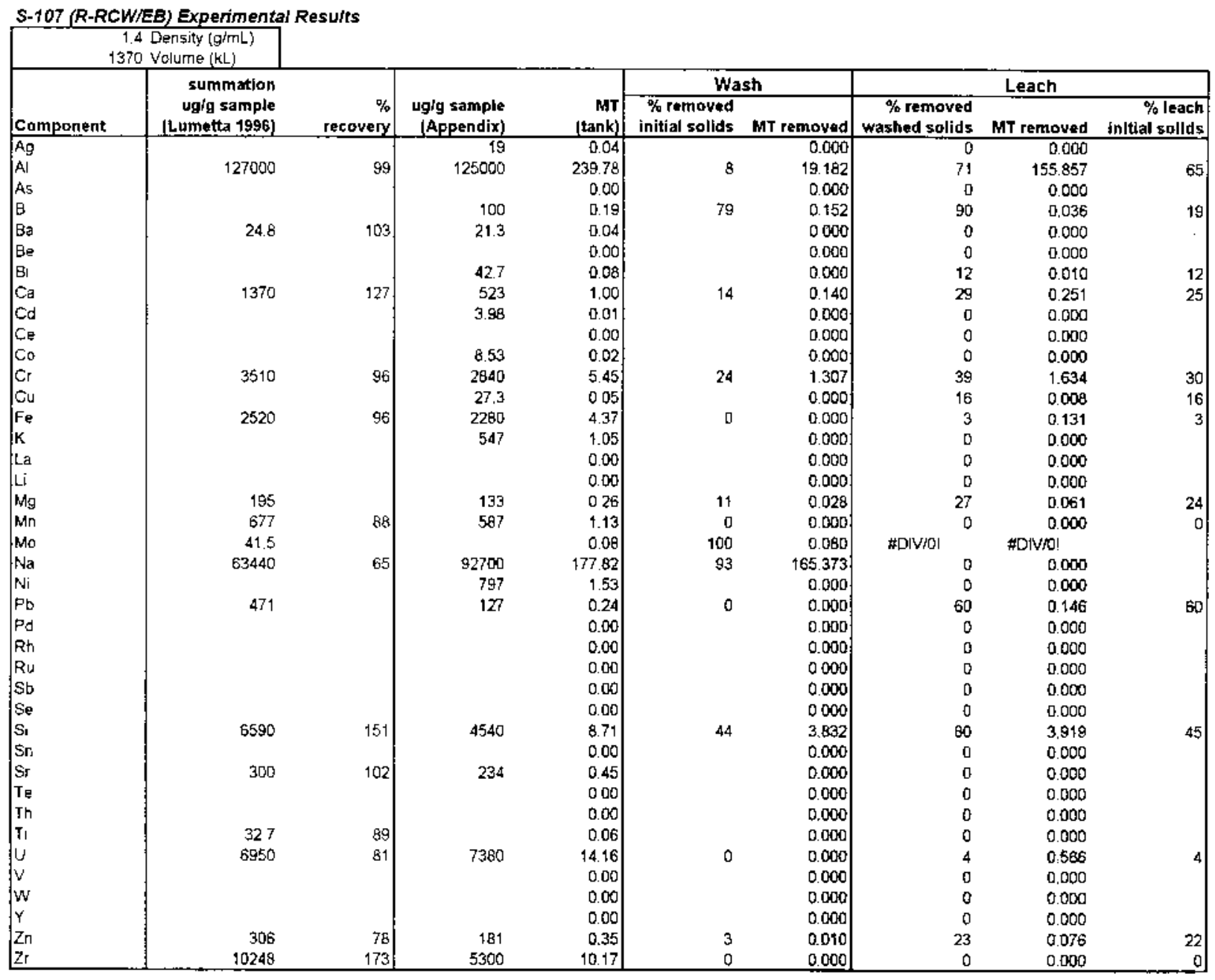

\begin{tabular}{|c|c|c|c|c|c|c|c|c|c|}
\hline \multirow[b]{2}{*}{ Component } & \multirow{2}{*}{$\begin{array}{r}\text { summation } \\
\text { ugig sample } \\
\text { (LLumetta 1996) }\end{array}$} & \multirow[b]{2}{*}{$\begin{array}{r}\% \\
\text { recovery }\end{array}$} & \multirow[b]{2}{*}{$\begin{array}{l}\text { ug/g sample } \\
\text { (Appendix) }\end{array}$} & \multirow[b]{2}{*}{$\begin{array}{r}\text { MT } \\
\text { (tank) }\end{array}$} & \multicolumn{2}{|c|}{ Wash } & \multicolumn{3}{|c|}{ Leach } \\
\hline & & & & & $\begin{array}{l}\text { \% removed } \\
\text { initial solids }\end{array}$ & MT removed & $\begin{array}{r}\% \text { removed } \\
\text { washed solids }\end{array}$ & WT removed & $\begin{array}{r}\% \text { removed } \\
\text { initial solids }\end{array}$ \\
\hline $\mathrm{OH}-$ & & & & 0.00 & & 0.000 & 0 & 0.000 & \\
\hline $\mathrm{NO3}-$ & & & 58000 & 111.26 & & 0.000 & 0 & 0.000 & \\
\hline NO2- & & & 31300 & 60.04 & & 0.000 & 0 & 0.000 & \\
\hline $\mathrm{PO} 4-3$ & 7880 & 293 & 6320 & 12.12 & 100 & 12.123 & \#DIVIO! & \#DIYo! & \\
\hline$\$ 04-2$ & & & 2790 & 5.35 & & 0.000 & 0 & 0.000 & \\
\hline F- & & & 14000 & 26.86 & & 0.000 & 0 & 0.000 & \\
\hline $\mathrm{Cl}$ & & & 1920 & 3.68 & & 0.000 & 0 & 0.000 & \\
\hline Br. & & & & 0.00 & & 0.000 & 0 & 0.000 & \\
\hline & $\begin{array}{r}\text { summalon } \\
\text { uCilg sample } \\
\text { [Lumetta 1996] }\end{array}$ & $\begin{array}{r}\% \\
\text { recovary }\end{array}$ & $\begin{array}{r}\text { uCilg sample } \\
\text { (Appendix) }\end{array}$ & $\begin{array}{r}c i \\
{[\tan k]}\end{array}$ & $\begin{array}{l}\text { \% removed } \\
\text { initial solids }\end{array}$ & Ci removed & $\begin{array}{l}\% \text { romoved } \\
\text { washed soljds }\end{array}$ & Ci removed & $\begin{array}{r}\% \text { removed } \\
\text { initial solids }\end{array}$ \\
\hline Total alpha & 1.01 & 96 & 1.13 & 2167.61 & 0 & 0.000 & 0 & 0.000 & 0 \\
\hline Pu-235, 240 & 0.508 & 93 & & 0.00 & 0 & 0000 & 0 & 0.000 & 0 \\
\hline$A m-241+P_{U}-238$ & 0.487 & 101 & & 000 & 0 & 0.000 & 0 & 0.000 & 0 \\
\hline Am-241 (gamma) & 0379 & 98 & 0.701 & 1344.68 & 0 & 0000 & 0 & 0.000 & 0 \\
\hline $\mathrm{Cm}-243,244$ & & 69 & & 0.00 & & 0.000 & 0 & 0.000 & 0 \\
\hline Cs-137 & 83.6 & 97 & 78.2 & 150006.21 & 77 & 115504783 & 100 & 34501.429 & 23 \\
\hline Co-60 & 0.0854 & 93 & 0093 & 178.40 & 0 & 0.000 & 3 & 5.352 & 3 \\
\hline Eu-154 & 0.112 & 99 & & 000 & 0 & 0.000 & 0 & 0.000 & 0 \\
\hline Eu-155 & 0.0726 & & & $0.0 \mathrm{r}$ & 0 & 0.000 & 0 & 0.000 & 0 \\
\hline$\$ r-90$ & 134 & 95 & 174 & 333773.41 & 0 & 0.000 & D & 0.000 & 0 \\
\hline Tc.99 & 0.0714 & 84 & & 136.96 & $9 \mathrm{~B}$ & 134.223 & 0 & 0.000 & 0 \\
\hline
\end{tabular}

Notes:

Characterization worksheet provided in Appendix

Lumetta G I. B M Rapko. M. J. Wagner, and J. LIu 1996 Washing and Caustic Leachung of Hanford Tank Situges: Resulfs of FY 1996 Studies PNNL-11278, Rev. 1, Pacific Northwest National Laboratory. Rıchland, Washington. 
T.104 (BIPO4 1C) LANL Experimental Resuirs

1.15 Density (g/mL)

1673 volume (kL)

\begin{tabular}{|c|c|c|c|c|c|c|c|c|c|}
\hline \multirow[b]{2}{*}{ Camponent } & \multirow{2}{*}{$\begin{array}{r}\text { summation } \\
\text { uglg sample } \\
\text { (Temer 1995) }\end{array}$} & \multirow[b]{2}{*}{$\begin{array}{r}\% \\
\text { recovery }\end{array}$} & \multirow[b]{2}{*}{$\begin{array}{r}\text { ugig sample } \\
\text { (Colton 1995) }\end{array}$} & \multirow[b]{2}{*}{$\begin{array}{r}M T \\
{[\tan k]}\end{array}$} & \multicolumn{2}{|c|}{ Wash } & \multicolumn{3}{|c|}{ Leach } \\
\hline & & & & & $\begin{array}{r}\% \text { removed } \\
\text { initial solids }\end{array}$ & MT removed & $\begin{array}{r}\% \text { removed } \\
\text { washed solids }\end{array}$ & MT removed & $\begin{array}{l}\% \text { leach } \\
\text { initial solids }\end{array}$ \\
\hline $\mathrm{Ag}$ & & & & 000 & & 0.000 & 5 & 0,000 & 5 \\
\hline $\mathrm{Al}^{2}$ & 15939.2 & 102 & 16200 & 3117 & 1 & 0.293 & 63 & 19.536 & 63 \\
\hline As & & & & 0.00 & & 0.000 & 36 & 0.000 & 36 \\
\hline 8 & & & 139 & 003 & & 0.000 & 0 & 0000 & \\
\hline $\mathrm{Ba}$ & 8.497 & 100 & 8.8 & 0.02 & 1 & 0000. & 0 & 0.000 & 0 \\
\hline $\mathrm{Be}$ & & & & 0.00 & & 0.000 & 0 & 0.000 & \\
\hline$\theta i$ & $\$ 5177.4$ & 127 & 18900 & 36.36 & 0 & 0.000 & 0 & 0.087 & 0 \\
\hline $\mathrm{Ca}$ & 147.086 & 125 & 664 & 1.28 & 4 & 0.045 & 6 & 0.070 & 6 \\
\hline $\mathrm{Cd}$ & & & 5.53 & 0.01 & 0 & 0.000 & 13 & 0.001 & 13 \\
\hline $\mathrm{Ce}$ & 160.564 & & & 0.31 & & 0.000 & t? & 0.000 & \\
\hline Co & 0.83212 & & 10.9 & 0.02 & 7 & 0.001 & 1 & 0.000 & 1. \\
\hline $\mathrm{Cr}$ & 735.43 & 117 & 916 & 1.76 & 17 & 0.300 & 40 & 0.585 & 33 \\
\hline $\mathrm{Cu}$ & 22.5903 & 144 & 46.9 & 0.09 & 0 & 0.000 & 32 & 0.029 & 32 \\
\hline $\mathrm{Fe}$ & & & 9020 & 17.35 & 0 & 0.000 & 0 & 0.000 & 0 \\
\hline K & 958.11 & 52 & 85.7 & 0.16 & & 0.000 & 0 & 0.000 & \\
\hline La & ‥6446 & 103 & & 0.00 & 0 & 0.000 & 0 & 0.000 & 0 \\
\hline $\mathrm{Li}$ & & & & 0.00 & & 0.000 & 0 & 0.000 & \\
\hline$M g$ & & & 159 & ‥31 & 0 & 0.000 & 0 & 0.000 & 0 \\
\hline$M n$ & & & 59.6 & 0.11 & 0 & 0.000 & 0 & 0.000 & 0 \\
\hline Mo & 2.2268 & 109 & & 0.00 & 71 & 0.003 & 70 & 0.001 & 20 \\
\hline $\mathrm{Na}$ & 158220 & 269 & 64500 & 124.08 & 25 & 31.271 & 95 & 88.391 & 71 \\
\hline $\mathrm{Ni}$ & & & 12.7 & 0.02 & 0 & 0.000 & 2 & 0.001 & 2 \\
\hline $\mathrm{Pb}$ & 48.638 & 113 & 61.2 & 0.12 & 0 & 0.000 & 2 & 0.003 & 2 \\
\hline Pd & & & & 0.00 & & 0.000 & 0 & 0.000 & \\
\hline $\mathrm{Rh}$ & & & & 0.00 & & 0.000 & 0 & 0.000 & \\
\hline Ru & 009376 & 80 & & 0.00 & 21 & 0.000 & 18 & 0.000 & 14 \\
\hline Sb & & & & 0.00 & & 0.000 & 0 & 0.000 & \\
\hline Se & & & & 0.00 & & 0.000 & 0 & 0.000 & \\
\hline $\mathrm{Si}$ & 3398.8 & 72 & 6440 & 12.39 & $d 2$ & 5.179 & 38 & 2.774 & 22 \\
\hline $\mathrm{Sn}$ & & & & 0.00 & & 0.000 & 86 & 0.000 & 86 \\
\hline Sr & 82333 & 105 & 96.8 & 0.19 & 0 & 0.000 & 0 & 0.000 & 0 \\
\hline Te & & & & 0.00 & & 0.000 & 마 & 0.000 & \\
\hline Th & 1.08703 & & & 0.00 & 0 & 0.000 & 0 & 0.000 & 0 \\
\hline $\mathrm{Ti}$ & & & & 0.00 & & 0.000 & 0 & 0.000 & \\
\hline U & 835 Q5 & 93 & 867 & 1.67 & 1 & 0010 & 14 & 0.233 & 14 \\
\hline V & & & & 0.00 & & 0,000 & 0 & 0.000 & \\
\hline$w$ & 0223559 & & & 0.00 & $\infty 0$ & 0.000 & 70 & 0.000 & 28 \\
\hline$Y$ & & & & 0.00 & & 0.000 & 0 & 0.000 & \\
\hline $\mathrm{Zn}$ & & & 152 & 0.29 & 0 & 0.000 & 32 & 0.095 & 32 \\
\hline $\mathrm{Zr}$ & 144156 & 94 & 69.1 & 0.13 & 1 & 0.001 & 0 & 0.000 & 0 \\
\hline
\end{tabular}

\begin{tabular}{|c|c|c|c|c|c|c|c|c|c|}
\hline \multirow[b]{2}{*}{ Component } & \multirow{2}{*}{$\begin{array}{r}\text { summation } \\
\text { uglg sample } \\
\text { (Temer 1995) }\end{array}$} & \multirow[b]{2}{*}{$\begin{array}{r}\% \\
\text { recovery }\end{array}$} & \multirow[b]{2}{*}{$\begin{array}{l}\text { ugig sample } \\
\text { (Colton 1995) }\end{array}$} & \multirow[b]{2}{*}{$\begin{array}{r}\text { MT } \\
\text { tank }\}\end{array}$} & \multicolumn{2}{|c|}{ Wash } & \multicolumn{3}{|c|}{ Leach } \\
\hline & & & & & $\begin{array}{l}\% \text { removed } \\
\text { initial solids }\end{array}$ & MT removed & $\begin{array}{l}\% \text { removed } \\
\text { washed solids }\end{array}$ & MT removed & $\begin{array}{l}\text { \% removed } \\
\text { initial solids }\end{array}$ \\
\hline $\mathrm{OH}$ & & & & 0.00 & & 0.000 & $\overline{0}$ & 0.000 & \\
\hline NO3- & & & 58000 & 111.59 & & 0.000 & 0 & 0.000 & \\
\hline NO2- & & & 4070 & 7.83 & & 0.000 & 0 & 0.000 & \\
\hline $\mathrm{PO} 4-3$ & 65200 & 110 & 73900 & 142.18 & 27 & 37748 & 100 & 104.500 & 74 \\
\hline $504-2$ & & & 3890 & 7.48 & 50 & 4.459 & 20 & 0.598 & 8. \\
\hline F- & & & 8580 & 16.54. & 27 & 4.457 & 98 & 11.869 & 72 \\
\hline Cl. & & & 670 & +29 & & 0.000 & 0 & 0.000 & \\
\hline $\mathrm{Br}-$ & & & & 0.00 & & 0.000 & 0 & 0,000 & \\
\hline & $\begin{array}{r}\text { summation } \\
\text { uCilg sample } \\
\text { [Temer 1995] }\end{array}$ & $\begin{array}{r}\% \\
\text { recovery }\end{array}$ & $\begin{array}{l}\text { uCilg sample } \\
\text { (Colton t995) }\end{array}$ & $\begin{array}{r}c i \\
(\tan k)\end{array}$ & $\begin{array}{l}\text { \% romoved } \\
\text { initial solids }\end{array}$ & Ci romaved & $\begin{array}{l}\text { \% removed } \\
\text { washed solids }\end{array}$ & Ci removed & $\begin{array}{l}\% \text { removed } \\
\text { in ttial sollids }\end{array}$ \\
\hline Total alphä & 0.60944 & 82 & & 1172.51 & 0 & 0.000 & 1 & 17.470 & 1 \\
\hline $\mathrm{Pu}-239,240$ & 019924 & 64 & & 383.32 & 0 & 0.000 & 0 & 0.000 & 0 \\
\hline $\mathrm{Am}-241+\mathrm{Pu}_{\mathrm{L}}-238$ & & & & 0.00 & & 0.000 & 0 & 0.000 & 0 \\
\hline Am-24t (gamma) & & & 0.017 & 32.71 & 0 & 0.000 & 0 & 0.000 & \\
\hline $\mathrm{Cm}-243,244$ & & & & 0.001 & & 0.000 & 0 & 0.000 & \\
\hline Cs-137 & $0+3478$ & 94 & & 259.31 & 12 & 31.895 & 18 & 41.489 & 16 \\
\hline Co.60 & & & & 0.00 & & 0.000 & 0 & 0.000 & \\
\hline$E_{k-154}$ & & & & 0.00 & & 0.000 & 0 & 0.000 & \\
\hline Ev-155 & & & & 0.00 & & 0.000 & 0 & 0.000 & \\
\hline $5 r-90$ & 123939 & 70 & 2.61 & 5021.42 & 0 & 0.000 & 0 & 0.000 & 0 \\
\hline$T_{c-99}$ & & & 0,001 & 1.92 & & 0.000 & 0 & 0000 & \\
\hline
\end{tabular}

Colton, N. G. 1995. Sludge Fretreatment Chemistry Evaluation: Enhanced Studge Washing Separation Factors. PNL-10512

Pacific Northwest National Laboratory. Richland Washington.

Temer, D.J and R. Villarreal 1995. Siudge Washing and Alkafine Leaching Jests on Aclual Hanford Tank Sludge. A Slatus Report. LALR 95-2070,

Los Alamos National Laboratory. Los Alamos. New Mexico. 
T-104 (BIPO4 1C) PNNL Experimental Results

\begin{tabular}{|c|c|c|c|c|c|c|c|c|c|}
\hline \multicolumn{10}{|c|}{$\begin{array}{r}1.15 \text { Density }(\mathrm{g} / \mathrm{mL}) \\
1673 \text { Volume }(\mathrm{kL}) \\
\end{array}$} \\
\hline \multirow[b]{2}{*}{ Component } & \multirow{2}{*}{$\begin{array}{r}\text { summation } \\
\text { ugig sample } \\
\text { (Rapko t995) }\end{array}$} & \multirow[b]{2}{*}{$\begin{array}{r}\% \\
\text { recovery }\end{array}$} & \multirow[b]{2}{*}{$\begin{array}{r}\text { ugig sample } \\
\text { \{Colton 1995) }\end{array}$} & \multirow[b]{2}{*}{$\begin{array}{r}M T \\
\{\operatorname{tank}\}\end{array}$} & \multicolumn{2}{|c|}{ Wash } & \multicolumn{3}{|c|}{ Leach } \\
\hline & & & & & $\begin{array}{l}\% \text { romaved } \\
\text { initial sofids }\end{array}$ & MT remaved & $\begin{array}{l}\% \text { removed } \\
\text { washed sollds }\end{array}$ & MT removed & $\begin{array}{l}\% \text { leach } \\
\text { initial solids }\end{array}$ \\
\hline $\mathrm{Ag}$ & & & & 0.00 & & 0.000 & 0 & 0.000 & \\
\hline $\mathrm{Al}$ & 12600 & 91 & 16200 & 31.17 & 1 & 0.312 & 62 & 19.012 & 61 \\
\hline$A_{5}$ & 26.7 & & & 0.00 & 0 & 0.000 & $5 \uparrow$ & 0.000 & 51 \\
\hline 8 & 163 & 25 & 139 & 0.03 & 2 & 0,001 & 0 & 0.000 & 0 \\
\hline $\mathrm{Ba}$ & 10.2 & & 8.8 & 0.02 & 0 & 0.000 & 0 & 0.000 & 0 \\
\hline $\mathrm{Be}$ & & & & 0.00 & & 0.000 & 0 & 0000 & \\
\hline$B i$ & 16100 & 105 & 18900 & 36.36 & 0 & 0.000 & 0 & 0.000 & 0 \\
\hline $\mathrm{Ca}$ & 357 & 94 & 664 & 1.28 & 23 & 0.294 & 5 & 0051 & 4 \\
\hline Cod & & & 553 & 0.01 & & 0.000 & 0 & 0.000 & \\
\hline $\mathrm{Ce}$ & 204 & 174 & & 0.000 & 0 & 0.000 & 0 & 0.000 & 0 \\
\hline $\mathrm{Co}$ & 437 & 50 & 109 & 002 & 17 & 0.004 & 0 & 0.000 & 0 \\
\hline $\mathrm{Cr}$ & 914 & 101 & 916 & 176 & 0 & 0000 & 10 & 0.176 & 10 \\
\hline $\mathrm{Cu}$ & 679 & 327 & 46.9 & 0,09 & & 0,000 & 0 & 0.000 & 0 \\
\hline $\mathrm{Fe}$ & 8610 & 105 & 9020 & 17.35 & 0 & 0.000 & 0 & 0.000 & 0 \\
\hline $\mathrm{K}$ & & & 85.7 & 0.16 & & 0.000 & 0 & D.,000 & \\
\hline La & & & & 0.00 & & 0.000 & 0 & 0.000 & \\
\hline $\mathrm{Li}$ & & & & 0.00 & & 0.000 & 0 & 0.000 & \\
\hline $\mathrm{Mg}$ & 73 & 83 & 159 & 0.31 & 0 & 0.000 & 0 & 0.000 & 0 \\
\hline $\mathrm{Mn}$ & 108 & 61 & 59.6 & 0.11 & 0 & 0.000 & 0 & 0,000 & 0 \\
\hline Mo & & & & 000 & & 0,000 & 0 & 0.000 & \\
\hline $\mathrm{Na}$ & & & 64500 & 124.09 & 72 & 89.347 & 0 & 0.000 & \\
\hline $\mathrm{Ni}$ & & & 12.7 & 0.02 & & 0.000 & 0 & 0.000 & \\
\hline $\mathrm{Pb}$ & 43.6 & 75 & 61.2 & 0.12 & 0 & 0.000 & 0 & 0.000 & \\
\hline $\mathrm{Pd}$ & & & & 000 & & 0,000 & 0 & 0.000 & \\
\hline $\mathrm{Rh}$ & & & & 0.00 & & 0.000 & 0 & 0.000 & \\
\hline Ru & & & & 0.00 & & 0.000 & 0 & 0.000 & \\
\hline Sb & & & & 0.00 & & 0.000 & 0 & 0.000 & \\
\hline Se & 14.6 & & & 0.03 & 0 & 0.000 & 0 & 0.000 & 0 \\
\hline $\mathrm{Si}$ & 5600 & 83 & 6440 & 12.39 & 0 & 0.000 & 1 & 0.124 & 1 \\
\hline Sn & & & & 0.00 & & 0.000 & 0 & 0.000 & \\
\hline $\mathrm{Sr}$ & 976 & & 98.8 & 0.19 & 0 & 0.000 & 0 & 0.000 & 0 \\
\hline Te & & & & 0.00 & & 0.000 & 0 & 0.000 & \\
\hline Th & & & & 0.00 & & 0.000 & 0 & 0.000 & \\
\hline $\mathrm{T}_{\mathrm{i}}$ & 137 & & & 0.03 & 0 & 0.000 & 0 & 0.000 & 0 \\
\hline บ & 1190 & & 867 & 167 & 1 & 0.017 & 8 & 0.133 & 8 \\
\hline$v$ & & & & 0.00 & & 0.000 & 0 & 0.000 & \\
\hline$W$ & & & & 0,00 & & 0.000 & 0 & 0.000 & \\
\hline$Y$ & & & & 0.00 & & 0.000 & 0 & 0.000 & \\
\hline $\mathrm{Zn}$ & 823 & & 152 & 0.29 & 46 & 0.135 & 13 & 0.020 & 7 \\
\hline $\mathrm{Zr}$ & 35.6 & & 691 & 013 & 0 & 0.000 & 1 & 0.001 & 1 \\
\hline
\end{tabular}

\begin{tabular}{|c|c|c|c|c|c|c|c|c|c|}
\hline \multirow[b]{2}{*}{ Component } & \multirow{2}{*}{$\begin{array}{r}\text { summation } \\
\text { ugig sample } \\
\text { \{Rapko 1995\} }\end{array}$} & \multirow[b]{2}{*}{$\begin{array}{r}\% \\
\text { recovery }\end{array}$} & \multirow[b]{2}{*}{$\begin{array}{r}\text { ugfg sample } \\
\text { (Colton 1995) }\end{array}$} & \multirow[b]{2}{*}{$\begin{array}{r}\text { MT } \\
{[\tan k)}\end{array}$} & \multicolumn{2}{|c|}{ Wash } & \multicolumn{3}{|c|}{ Leach } \\
\hline & & & & & $\begin{array}{l}\% \text { removed } \\
\text { initial soljds }\end{array}$ & MT removed & $\begin{array}{l}\% \text { removed } \\
\text { washed solids }\end{array}$ & MT removed & $\begin{array}{l}\text { \% remaved } \\
\text { initial solids }\end{array}$ \\
\hline $\mathrm{OH}-$ & & & & 0.00 & & 0.000 & 0 & 0,000 & \\
\hline NO3- & & & 58000 & 111.59 & & 0.000 & 0 & 0.000 & \\
\hline $\mathrm{NO} 2$ & & & 4070 & 7.83 & & 0.000 & 0 & 0.000 & \\
\hline $\mathrm{PO} 4-3$ & 60400 & 100 & 73900 & 142.18 & 26 & 36.966 & 97 & 102.368 & 72 \\
\hline $504-2$ & 3580 & & 3890 & 7.48 & 89 & 6661 & 0 & 0.000 & \\
\hline F. & 7090 & & 8580 & 16.51 & 57 & 9.409 & 100 & 7.098 & 43 \\
\hline $\mathrm{Cl}$ & 853 & & 670 & 1.29 & & 0000 & 0 & 0.000 & \\
\hline \multirow[t]{2}{*}{ Br- } & & & & 000 & & 0.000 & 0 & 0.000 & \\
\hline & $\begin{array}{r}\text { Summation } \\
\text { uCi/g satnple } \\
\text { (Rapko 1995) }\end{array}$ & $\begin{array}{r}\% \\
\text { recavery }\end{array}$ & $\begin{array}{l}\text { ucíg sample } \\
\text { (Colton 1995) }\end{array}$ & $\begin{array}{r}c i \\
\{\tan k\} \\
\end{array}$ & $\begin{array}{l}\text { \% removed } \\
\text { initial solids }\end{array}$ & Ci removed & $\begin{array}{r}\text { \% removed } \\
\text { washed solids }\end{array}$ & Cl removed & $\begin{array}{l}\% \text { removed } \\
\text { initial solids }\end{array}$ \\
\hline Tatal alpha & 0.187 & 122 & & 359.77 & 0 & 0.000 & 0 & 0.000 & 0 \\
\hline$P u-239.240$ & 0.156 & 142 & & 300.13 & 0 & 0.000 & 0 & 0.000 & 0 \\
\hline $\mathrm{Am}=241+\mathrm{Pu}-238$ & 0.027 & 66 & & 51.95 & 0 & 0.000 & 0 & 0.000 & 0 \\
\hline Amr-241 (gamma) & 0.0261 & 124! & 0.017 & 32.71 & 0 & 0.000 & 0 & 0.000 & 0 \\
\hline $\mathrm{Cm}-243,244$ & & & & 0.00 & & 0.000 & 0 & 0.000 & \\
\hline C5-137 & 0.181 & 26 & & 348.23 & 0 & 0,000 & 69 & 240.278 & 69 \\
\hline Co-60 & & & & 0.00 & & 0.000 & 0 & 0.000 & \\
\hline$E u-154$ & & & & 0.00 & & 0.000 & 0 & 0.000 & \\
\hline Eu-155 & & & & 0.00 & & 0.000 & 0 & 0.000 & \\
\hline Sr-90 & 3.78 & 103 & 2.61 & 5021.42 & 0 & 0.000 & 1 & 50.214 & 1 \\
\hline Jc-99 & 0.0001 & & 0.001 & 1.92 & 0 & 0.000 & 0 & 0.000 & 0 \\
\hline
\end{tabular}

Na measurement impracucal due to method of sample prep (Rapko 1995): therefore. Na wash factor based on Colton 1995.

PO4 leach factor based on Rapko 1996

Colton. N. G. 1995 Situdge Pretrealment Chemisoy Evaltuation: Enhanced Sfudge Washing Separation Factors, PNL-10512.

Pacific Northwest National Laboratory, Rıchland Wașington

Rapko, B. M. G J. Lumetta, M JWagner 1995. Washing and Caustic Leaching of Hantord Tank Situdges. Resusts of FY1995 Studies, PNL-10712, Pacific Northwest Natıonal Laboratory. Rıchland Washington. 


\begin{tabular}{|c|c|c|c|c|c|c|c|c|c|}
\hline \multicolumn{10}{|c|}{$\begin{array}{c}15 \text { Density }(\mathrm{g} / \mathrm{mL}) \\
655 \text { Volume }(\mathrm{kL})\end{array}$} \\
\hline \multirow[b]{2}{*}{ Component } & \multirow{2}{*}{$\begin{array}{l}\text { summation } \\
\text { ug/g sample } \\
\text { (Temer 1995) }\end{array}$} & \multirow[b]{2}{*}{$\begin{aligned} \% \\
\text { recovery }\end{aligned}$} & \multirow[b]{2}{*}{$\begin{array}{r}\text { ug/g sample } \\
\text { (Coltan 1995) }\end{array}$} & \multirow[b]{2}{*}{$\begin{array}{r}\text { MT } \\
{[\operatorname{tank}]}\end{array}$} & \multicolumn{2}{|c|}{ Wash } & \multicolumn{3}{|c|}{ Leach } \\
\hline & & & & & $\begin{array}{l}\text { \% removed } \\
\text { initia! sollds }\end{array}$ & MT removed & $\begin{array}{r}\text { \% removed } \\
\text { washed solids }\end{array}$ & MT removed & $\begin{array}{r}\% \text { leach } \\
\text { initial solids }\end{array}$ \\
\hline $\mathrm{Ag}$ & 0.82 & & & 0.00 & 45 & 0000 & 5 & 0.000 & 3 \\
\hline $\mathrm{Al}$ & 30900 & 109 & 16300 & 16.01 & 4 & 0570 & 77 & 11.910 & 74 \\
\hline As & & & & 0.00 & 28 & 0.000 & 0 & 0.000 & \\
\hline$B$ & & & 13 & 001 & 44 & 0.006 & 17 & 0.001 & 9 \\
\hline Ba & 46 & & & 0.05 & 0 & 0.000 & 0 & 0.000 & 0 \\
\hline Be & & & & 0.00 & & 0000 & 0 & 0.000 & \\
\hline $\mathrm{Bi}$ & 13500 & & 12000 & 11.79 & 0 & 0.004 & 0 & 0.041 & 0 \\
\hline $\mathrm{Ca}$ & 635 & 111 & 761 & 0.75 & 0 & 0.000 & 0 & 0.000 & 0 \\
\hline $\mathrm{Cd}$ & & & & 0.00 & 0 & 0.000 & 0 & 0.000 & D \\
\hline$C_{e}$ & & & & 0.00 & & 0.000 & 0 & 0000 & \\
\hline Co & & & & 0.00 & & 0.000 & 0 & 0.000 & \\
\hline $\mathrm{Cr}$ & 337 & 94 & 360 & 0.35 & 42 & 0.148 & 32 & 0.066 & 19 \\
\hline $\mathrm{Cu}$ & & & & 0.00 & 0 & 0,000 & 4 & 0.000 & t \\
\hline $\mathrm{Fe}$ & 26300 & 116 & 31500 & 30.94 & 0 & 0.025 & 0 & 0.006 & D \\
\hline$k$ & & & 315 & 0.31 & & 0.000 & 0 & 0.000 & \\
\hline La & & & & 0.00 & ㅁ & 0.000 & 0 & 0.000 & 0 \\
\hline $\mathrm{Li}$ & & & & 0.00 & & 0.000 & 0 & 0.000 & \\
\hline $\mathrm{Mg}$ & & & 226 & 0.22 & 0 & 0.000 & 0 & 0.000 & 0 \\
\hline $\mathrm{Mn}$ & & & 221 & 0.22 & 0 & 0.000 & 0 & 0.000 & D: \\
\hline Mo & 118 & & & 0.01 & 89 & 0.010 & 84 & 0.001 & 9] \\
\hline Na & 82000 & 68 & 130000 & . 127.69 & 51 & 65.120 & 78 & 48.559 & 38 \\
\hline N: & & & 290 & 0.28 & 1 & 0.002 & 0 & 0.000 & 0 \\
\hline Po & 565 & $\Leftrightarrow G$ & 796 & 0.78 & 0 & 0.000 & 1 & 0.019 & i. \\
\hline $\mathrm{Pd}$ & 209 & & & 0.00 & 16 & 0.000 & 12 & 0.000 & 10 \\
\hline $\mathrm{Rh}$ & & & & 0.0 .5 & & 0000 & 0 & 0.000 & \\
\hline $\mathrm{Ru}$ & 16.3 & & & 002 & 44 & 0.007 & 9 & 0.001 & 5 \\
\hline $\mathrm{sb}$ & & & & 000 & & 0.000 & 0 & 0.000 & \\
\hline se & & & & 0.00 & & 0.000 & 0 & 0000 & \\
\hline $\mathrm{si}$ & 5000 & 62 & 6060 & 5.95 & 28 & 1.649 & 6 & 0.276 & $5 t$ \\
\hline sin & & & & 0.00 & & 0.000 & 0 & 0.000 & \\
\hline $\mathrm{S}_{\mathrm{r}}$ & 835 & 110 & 962 & 0.94 & 0 & 0.000 & 0 & 0.006 & 0 \\
\hline Te & & & & 0.00 & & 0.000 & 0 & 0.006 & \\
\hline$T h$ & & & & 0.00 & & 0.000 & 0 & 0.000 & \\
\hline $\mathrm{Tt}$ & & & & 0.00 & & 0.000 & 0 & 0.000 & \\
\hline U & 18800 & 94 & 25400 & 24.95 & 4 & 0.943 & 0 & 0.077 & 0 \\
\hline iv & & & & 0.00 & & 0.000 & 0 & 0.000 & \\
\hline$w$ & 63.5 & & & 0.06 & & 0.000 & 40 & 0.025 & 40 \\
\hline$Y$ & & & & 0.00 & & 0.000 & 0 & 0.000 & \\
\hline$z n$ & & & & 0.00 & & 0.000 & 0 & 0.000 & \\
\hline $\bar{Z} r$ & & & 93.2 & 0.09 & 1 & 0.001 & 0 & 0.000 & 0 \\
\hline
\end{tabular}

\begin{tabular}{|c|c|c|c|c|c|c|c|c|c|}
\hline \multirow[b]{2}{*}{ Component } & \multirow{2}{*}{$\begin{array}{c}\text { summation } \\
\text { ugig sample } \\
\text { (Temer 1995\} }\end{array}$} & \multirow[b]{2}{*}{$\begin{array}{r}\% \\
\text { recovary }\end{array}$} & \multirow[b]{2}{*}{$\begin{array}{l}\text { ug/g sample } \\
\text { (Colton 1995) }\end{array}$} & \multirow[b]{2}{*}{$\begin{array}{r}M T \\
{[\operatorname{tank}]}\end{array}$} & \multicolumn{2}{|c|}{ Wash } & \multicolumn{3}{|c|}{ Leach } \\
\hline & & & & & $\begin{array}{l}\text { \% removed } \\
\text { initial solids }\end{array}$ & MT removed & $\begin{array}{l}\text { \% removed } \\
\text { washed solids }\end{array}$ & MT remaved & $\begin{array}{c}\text { \% ramoved } \\
\text { initial solids }\end{array}$ \\
\hline $\overrightarrow{\mathrm{OH}}$ & & & & 0.00 & & 0.000 & 可 & 0.000 & \\
\hline NO3. & & & 74500 & 73.17 & & 0.000 & 0 & 0.000 & \\
\hline NO2- & & & 11700 & 11.49 & & 0.000 & 0 & 0.000 & \\
\hline $\mathrm{PO} 4-3$ & 84700 & 104 & 96200 & 94.49 & 85 & 80.315 & 87 & 12.397 & 13 \\
\hline$S O 4-2$ & 9750 & 71 & 10600 & 10.41 & 79 & 8.225 & 0 & 0.000 & 0 \\
\hline F- & 5750 & 127 & 11400 & 11.20 & 87 & 9.742 & 0 & 0.000 & 0 \\
\hline C:- & & & 540 & 0.53 & & 0,000 & 0 & 0,000 & \\
\hline Br. & & & & i. 00 & & 0.000 & 0 & 0.000 & \\
\hline & $\begin{array}{r}\text { summation } \\
\text { uCitg sample } \\
\text { (Temer 1995) }\end{array}$ & $\begin{array}{r}\% \\
\text { recovery }\end{array}$ & $\begin{array}{l}\text { uCilg sample } \\
\text { (Colton 1995) }\end{array}$ & $\begin{array}{r}c i \\
(\tan k)\end{array}$ & $\begin{array}{l}\% \text { removed } \\
\text { initial solids }\end{array}$ & Ci remaved & $\begin{array}{l}\% \text { romoved } \\
\text { washed solids }\end{array}$ & Ci removed & $\begin{array}{r}\% \text { removed } \\
\text { initial solids }\end{array}$ \\
\hline Iotal alpha & 2.26 & 67 & & 0.00 & 0 & 0.000 & 0 & 0.000 & 0 \\
\hline$P u-239,240$ & 028 & 27 & 0.152 & 149.30 & 0 & 0.000 & 0 & 0.000 & 0 \\
\hline$A_{\mathrm{Mm}}-241+\mathrm{PU}-238$ & & & & 0.00 & 0 & 0.000 & 0 & 0.000 & 0 \\
\hline Am-241 (gamma) & 001 & 54 & 0.014 & 13.75 & & 0.000 & 0 & 0.000 & \\
\hline $\mathrm{Cm}-243,244$ & & & & 0.00 & & 0.000 & 0 & 0.000 & \\
\hline C5-137 & 64 & 82 & 21.5 & 21117.46 & 25 & 5226.572 & 88 & 13918.519 & 66 \\
\hline Co-60 & & & & 0.00 & & 0.000 & 0 & 0.000 & \\
\hline Eu-154 & & & & 0.00 & & 0.000 & 0 & 0.000 & \\
\hline Eu-155 & & & & 0.00 & & 0.000 & 0 & 0.000 & \\
\hline Sr-90 & 103 & 118 & 109 & 107060.62 & 0 & 53.530 & 0 & 0.000 & 0 \\
\hline$T_{c-9 G}$ & & & 0.051 & 50.09 & 83 & 41.507 & 0 & 0.000 & \\
\hline
\end{tabular}

Colten, N G. 1995. Sludge Fretreatment Chemistry Evaluation. Enhanced Studge Washing Separation Factors. PNL-10512,

Pacific Northwest National Laboratory. Richland Washıngton.

Temer. D J. and R Villarreal 1995. Situdge Washing and Aikaline Leaching Tesis on Actual Hantord Tank Siudge: A Status Report, LAUR 95-2070.

Los Alamos National Laboratory. Los Alamos New Mexico 
T-111 (BjPO42C, BiP04 224) Experimental Resuits

\begin{tabular}{|c|c|c|c|c|c|c|c|c|c|}
\hline & $\begin{array}{l}\text { Density }\left(\mathrm{g}^{\prime} \mathrm{mL}\right) \\
\text { Volume }(\mathrm{kL})\end{array}$ & & & & & & & & \\
\hline & summation & & & & Wa & & & Leach & \\
\hline Component & $\begin{array}{l}\text { ugig sample } \\
\text { (Rapko 1995) }\end{array}$ & $\begin{array}{r}\% \\
\text { recovery }\end{array}$ & $\begin{array}{l}\text { ugig sample } \\
\text { (Colton 1995\} }\end{array}$ & $\begin{array}{r}M T \\
\{\tan k\}\end{array}$ & $\begin{array}{l}\% \text { removed } \\
\text { initial solids }\end{array}$ & MT remnved & $\begin{array}{l}\% \text { removed } \\
\text { washod solids }\end{array}$ & MT removed & $\begin{array}{r}\% \text { leach } \\
\text { initial solids }\end{array}$ \\
\hline $\mathrm{Ag}$ & 57.6 & 120 & & 0.11 & 2 & 0.002 & 0 & 0.000 & 0 \\
\hline $\mathrm{Al}$ & 883 & 75 & 570 & 1.08 & 0 & 0.000 & 13 & 0.140 & 13 \\
\hline As & 269 & & & 005 & 0 & 0.000 & 0 & 0000 & 0 \\
\hline$B$ & 442 & & 321 & 0.06 & 60 & 0.036 & 18 & 0.004 & 7 \\
\hline Ba & 44.4 & 93 & 69 & 0.13 & 0 & 0.000 & 0 & 0.000 & 0 \\
\hline Be & & . & & 000 & & 0.000 & 0 & 0.000 & \\
\hline $\mathrm{E}$ & 14800 & 98 & 25900 & 48.65 & 0 & 0.000 & 0 & 0.000 & 0 \\
\hline $\mathrm{Ca}$ & 2980 & 78 & 2420 & 456 & 0 & 0.000 & 1 & 0.046 & 1 \\
\hline $\mathrm{Cd}$ & 8.06 & 67 & & 0.02 & 0 & 0.000 & 0 & 0.000 & 0 \\
\hline $\mathrm{Ce}$ & 269 & & & 005 & 0 & 0.000 & 0 & 0.000 & 0 \\
\hline Co & 806 & 34 & 11.5 & 002 & 0 & 0000 & 0 & 0.000 & 0 \\
\hline $\mathrm{Cr}$ & 1180 & 109 & 1970 & 372 & 24 & 0.892 & 53 & 1.486 & 40 \\
\hline $\mathrm{Cu}$ & 349 & 4 & 33.6 & 0.06 & 0 & 0,000 & 5 & 0.003 & 5 \\
\hline $\mathrm{Fe}$ & 17500 & 122 & 18500 & 34.89 & 0 & 0.0000 & 0 & 0.000 & 0 \\
\hline$k$ & & & 1130 & 2.13 & & 0.000 & t & 0.000 & \\
\hline La & 2690 & 122 & 4220 & 7.96 & 0 & 0000 & 0 & 0.000 & 0 \\
\hline $\mathrm{L}_{1}$ & & & & 0.00 & & 0.000 & 0 & 0.000 & \\
\hline $\mathrm{Mg}$ & 566 & 118 & 377 & 0.71 & 0 & 0.000 & 0 & 0.000 & 0 \\
\hline$M$ & 12900 & 102 & 6330 & 11.94 & 0 & 0.000 & 0 & 0.000 & 0 \\
\hline Mo & 538 & & & 0.01 & 0 & 0.000 & 0 & 0,000 & 0 \\
\hline $\mathrm{Na}$ & & & 37000 & 69.78 & 89 & 62.108 & 0 & 0.000 & \\
\hline $\mathrm{Ni}$ & & & 131 & 0.25 & & 0.000 & 0 & 0.000 & \\
\hline $\mathrm{Pb}$ & 478 & 100 & 365 & 0.69 & 0 & 0.000 & 2 & 0.014 & 2 \\
\hline Pod & & & & 0.00 & & 0.000 & 0 & 0.000 & \\
\hline Rh & 135 & & & 0.03 & 0 & 0.000 & 0 & 0.000 & 0 \\
\hline Ru & & & & 0.00 & & 0.000 & 0 & 0.000 & \\
\hline Sb & & & & 0.00 & & 0.000 & 0 & 0.000 & \\
\hline $\mathrm{Se}$ & 538 & & & 0.00 & 0 & 0.000 & 0 & 0.000 & 0 \\
\hline SI & 11300 & 100 & 5670 & 10.69 & 20 & 2.139 & 50 & 4.278 & 40 \\
\hline $\mathrm{Sn}$ & 135 & & & 0.25 & 0 & 0.000 & 0 & 0.000 & 0 \\
\hline Si & 175 & 107 & 298 & 0.56 & 0 & 0.000 & 0 & 0.000 & 0 \\
\hline Te & & & & 0.00 & & 0.000 & 0 & 0.000 & \\
\hline Th & & & & 0.00 & & 0.000 & 0 & a.DO0 & \\
\hline $\mathrm{Ti}$ & 148 & 171 & & 0.28 & 0 & 0.000 & 0 & 0.000 & 0 \\
\hline U & 5420 & 100. & 2800 & 5.28 & 0 & 0000 & 2 & 0.106 & 2 \\
\hline$v$ & 0.237 & & & 0.00 & 0 & 0.000 & 100 & 0.000 & 100 \\
\hline$w$ & 135 & & & 0.25 & 0 & 0.000 & 0 & 0.000 & 0 \\
\hline$Y$ & & & & 0.00 & & 0.000 & 0 & 0.000 & \\
\hline $\mathrm{Zn}$ & 151 & 48. & 106 & 0.20 & 0 & 0.000 & 27 & 0.054 & 27 \\
\hline$Z r$ & 16.1 & & 4 & 0.01 & 0 & 0000 & 0 & 0.000 & 0 \\
\hline
\end{tabular}

\begin{tabular}{|c|c|c|c|c|c|c|c|c|c|}
\hline \multirow[b]{2}{*}{ Component } & \multirow{2}{*}{$\begin{array}{r}\text { summation } \\
\text { ugig sample } \\
\text { (Rapko 1995) }\end{array}$} & \multirow[b]{2}{*}{$\begin{array}{r}\% \\
\text { recovery }\end{array}$} & \multirow[b]{2}{*}{$\begin{array}{r}\text { ugig sample } \\
\text { \{Colton } 1995\}\end{array}$} & \multirow[b]{2}{*}{$\begin{array}{r}\text { MT } \\
\text { (tank) }\end{array}$} & \multicolumn{2}{|c|}{ Wash } & \multicolumn{3}{|c|}{ Leach } \\
\hline & & & & & $\begin{array}{l}\% \text { ramoved } \\
\text { initial solids }\end{array}$ & MT removid & $\begin{array}{l}\text { \% removed } \\
\text { washed sollds }\end{array}$ & MT removed & $\begin{array}{l}\% \text { removed } \\
\text { Initial solids }\end{array}$ \\
\hline$\widehat{\mathrm{OH}-}$ & & & & 0.00 & & 0.000 & 0 & 0.000 & \\
\hline NO3. & & & $\$ 1300$ & 77.89 & & 0.000 & 0 & 0.000 & \\
\hline $\mathrm{NO}_{2}-$ & & & 793 & 1.50 & & 0.000 & 0 & 0.000 & \\
\hline PO4-3 & 18900 & 101 & 31300 & 59.03 & 50 & 29.517 & 46 & 13.578 & 23 \\
\hline $504-2$ & 4130 & 86 & 3670 & 6.92 & 64 & 4.430 & 0 & 0,000 & \\
\hline F. & & & 2310 & 4.36. & & 0.000 & 0 & 0.000 & \\
\hline $\mathrm{Cl}-$ & & & 460 & 0.85 & & 0.000 & 0 & 0.000 & . \\
\hline Br- & & & & 0.00 & & 0.000 & 0 & 0.000 & \\
\hline & $\begin{array}{l}\text { summation } \\
\text { uC } 1 / g \text { sample } \\
\text { (Rapko } 2995 \text { ) }\end{array}$ & $\begin{array}{r}\% \\
\text { recovery }\end{array}$ & $\begin{array}{l}\text { uCíg sample } \\
\text { (Colton 1995) }\end{array}$ & $\begin{array}{r}\mathrm{Cl} \\
(\tan \mathrm{k})\end{array}$ & $\begin{array}{l}\text { \% ramovid } \\
\text { initial solids }\end{array}$ & Ci removed & $\begin{array}{r}\text { X removed } \\
\text { wasthed solids }\end{array}$ & Ci removed & $\begin{array}{l}\% \text { removed } \\
\text { |mitlal solids }\end{array}$ \\
\hline Total alpha & 0.42 & 103 & & 792.15 & 0 & 0.000 & 0 & 0.000 & 0 \\
\hline Pu-239, 240 & 0.384 & 107 & 0.139 & 262.16 & 0 & 0.000 & 0 & 0.000 & 0 \\
\hline$A m-249+P u-238$ & 0.0358 & 65 & & 67.52 & 0 & 0.000 & 0 & 0.000 & 0 \\
\hline Am-241 (gamma) & 0.0638 & 106 & 0.043 & 81.10 & 0 & 0.000 & 0 & 0.000 & 0 \\
\hline $\mathrm{Cm}-243,244$ & & & & 0.00 & & 0.000 & 0 & 0.000 & \\
\hline Cs -137 & 0.326 & 198 & 0.166 & 313.09 & 25 & 78.272 & 41 & 97.057 & 31 \\
\hline Co-f́n & & & & 0.00 & & 0.000 & 0 & 0.000 & \\
\hline Eu- $\{54$ & & & & 0.00 & & 0.000 & 0 & 0.000 & \\
\hline Eu- 155 & & & & 0.00 & & 0.000 & 0 & 0.000 & \\
\hline Sr.90 & 10.8 & 109 & 5.42 & 10222.48 & 0 & 0.000 & 0 & 0.000 & 0 \\
\hline tc-99 & 0.0003 & & & 0.57 & 12 & 0.058 & 0 & 0.000 & 0 \\
\hline
\end{tabular}

Na measurement impractical due to method of sample prep (Rapko 1995); therefore. Na wash factor based on Colton 1995.

Anion measurements could not be accurately determined due to matrix effects (Rapko 1985)

Colton, N G 1995 Sludge Pretreatment Chemistry Evaluation: Enhanced Studge Washing Separation Factors, PNL-10512.

Pacific Northwest National Laboratory. Richland Washington.

Rapko. B M. G. J. Lumetta, M J. Wagner 1995 Washing and Caustic Leaching of Hantord Tank Situges: Resutts of FY 1995 Studies, PNL-10712.

Pacific Northwest National Laboratory. Richland Washingten. 
TY-104 [TBP, BiPO4 1C(I)] Experimental Results

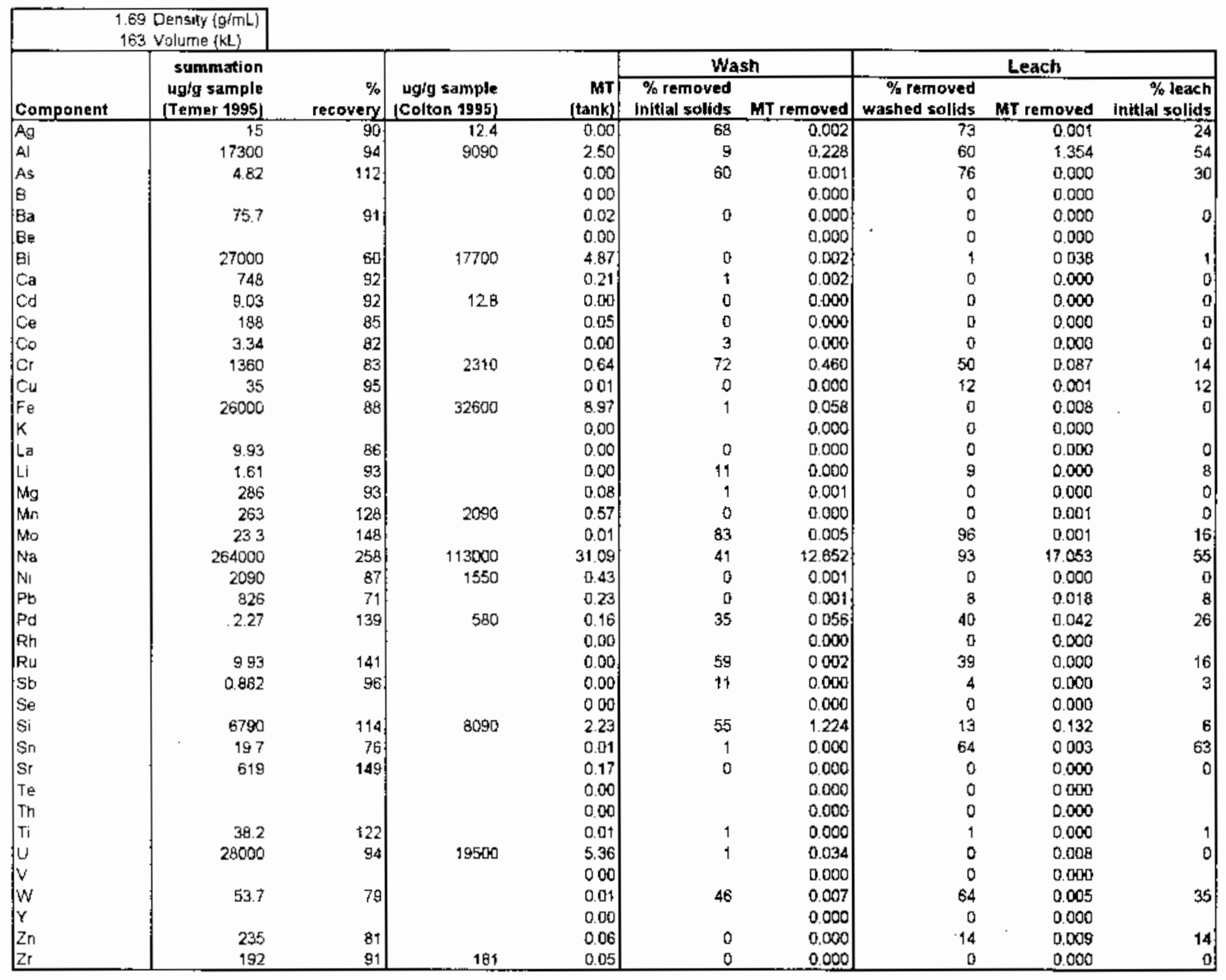

\begin{tabular}{|c|c|c|c|c|c|c|c|c|c|}
\hline \multirow[b]{2}{*}{ Component } & \multirow{2}{*}{$\begin{array}{r}\text { summation } \\
\text { ugig sample } \\
\text { (Temer 1995) }\end{array}$} & \multirow[b]{2}{*}{$\begin{array}{r}\% \\
\text { recovary }\end{array}$} & \multirow[b]{2}{*}{$\begin{array}{r}\text { ugig sample } \\
\text { (Colton 1995) }\end{array}$} & \multirow[b]{2}{*}{$\begin{array}{r}\text { MT } \\
\text { (tank) }\end{array}$} & \multicolumn{2}{|c|}{ Wash } & \multicolumn{3}{|c|}{ Leach } \\
\hline & & & & & $\begin{array}{l}\text { \% femoved } \\
\text { initial solids }\end{array}$ & MT removed & $\begin{array}{l}\% \text { removed } \\
\text { washed solids }\end{array}$ & MT removed & $\begin{array}{l}\% \text { removed } \\
\text { intitial salids }\end{array}$ \\
\hline$\overline{\mathrm{OH}}$ & & & 4290 & 0.35 & & 0.000 & 0 & 0.000 & \\
\hline NO3- & & & 46600 & 12.82 & & 0.000 & 0 & 0.000 & \\
\hline NO2- & & & 12200 & 3.36 & & 0.000 & 0 & 0.000 & \\
\hline $\mathrm{PO} 4.3$ & 83300 & 160 & 78600 & 21.62 & 83 & 18.005 & 19 & 0.688 & 3 \\
\hline $504-2$ & 3450 & 121 & 5230 & 1.44 & 90 & 1.304 & 0 & O.DOG & \\
\hline $\mathrm{F}-$ & 7090 & 193 & 5350 & 1.47 & 97 & 1.433 & 50 & 0.020 & 1 \\
\hline cl- & & & 598 & 0.16 & & 0.000 & 0 & 0,000 & \\
\hline Er- & & & & 0.00 & & 0.000 & 0 & 0.000 & \\
\hline & $\begin{array}{l}\text { summation } \\
\text { uCitg sample } \\
\text { [Temer } 1995 \text { ] }\end{array}$ & $\begin{array}{r}\% \\
\text { recovery }\end{array}$ & $\begin{array}{l}\text { uCifg sample } \\
\text { (Colton 1995) }\end{array}$ & $\begin{array}{r}c i \\
\text { [tank) }\end{array}$ & $\begin{array}{l}\text { \% removed } \\
\text { initial solids }\end{array}$ & Ci removed & $\begin{array}{l}\% \text { removed } \\
\text { washed solids }\end{array}$ & Ci removed & $\begin{array}{l}\% \text { removed } \\
\text { Initial solids }\end{array}$ \\
\hline Total alpha & & & & 0.00 & & 0.000 & 0 & 0.000 & \\
\hline Pu.239 240 & 0.176 & & 0.181 & 49.79 & 0 & 0.000 & 0 & 0,000 & 0 \\
\hline $\mathrm{A}_{\mathrm{N}} \mathrm{-}-241+\mathrm{Pu}-23 \mathrm{~B}$ & & & & 0.00 & & 0.000 & D & 0.000 & \\
\hline Am-241 (gamma) & & & 0.034 & 9.35 & 0 & 0.000 & 0 & 0.000 & 0 \\
\hline $\mathrm{Cm}-243,244$ & & & & 0.00 & & 0.000 & 0 & 0.000 & \\
\hline Cs.137 & 50.3 & & 45.4 & 12489.07 & 41 & 5080.556 & 22 & 1598.602 & 13 \\
\hline $\mathrm{Co}-6 \mathrm{C}$ & & & 0.023 & 6.33 & & 0.000 & 0 & 0.000 & \\
\hline Ev-154 & & & & 0.00 & & 0.000 & 0 & 0.000 & \\
\hline Eu-155 & & & & 0.00 & & 0.000 & 0 & 0.000 & \\
\hline Sr-90 & 144 & & 132 & 36311.85 & 0 & 0.000 & 0 & 0.000 & 0 \\
\hline Tc-5g & & & 0033 & 9.08 & 0 & 0.000 & 0 & 0.000 & 은 \\
\hline
\end{tabular}

Coton, N G 1995 Siludge Pretreatment Chemistry Evaluation: Enhanced Studge Washing Separation Fatclors. PNL-10512.

Pactic Northwest National Laboratory, Richland Washington.

Temer. D. I and R. Vallarreal. 1996. Studge Washing and Alkaline Leaching Tests on Actuat Hantord Tank Sludge: FY 1996 Results,

LAUR96.2839, Los Alamos National Laboratory. Los Alamos, New Maxico 


\begin{tabular}{|c|c|c|c|c|c|c|c|c|c|}
\hline \multirow[b]{3}{*}{ Component } & \multicolumn{9}{|c|}{$\begin{array}{l}1.5 \text { Density }\{\mathrm{g} / \mathrm{mL}) \\
704 \text { Volume }\{\mathrm{kL}\}\end{array}$} \\
\hline & \multirow{2}{*}{$\begin{array}{r}\text { range } \\
\text { uglg sample } \\
\text { [Lumetta 1994] }\end{array}$} & \multirow[b]{2}{*}{$\begin{array}{r}\% \\
\text { recovery }\end{array}$} & \multirow[b]{2}{*}{$\begin{array}{l}\text { ugig sample } \\
\text { (Colton 1995) }\end{array}$} & \multirow[b]{2}{*}{$\begin{array}{r}M 7 \\
{[\tan k]}\end{array}$} & \multicolumn{2}{|c|}{ Wash } & \multicolumn{3}{|c|}{ Leach } \\
\hline & & & & & $\begin{array}{l}\% \text { removed } \\
\text { Initial solids }\end{array}$ & MT removed & $\begin{array}{r}\% \text { removed } \\
\text { washed solids }\end{array}$ & MT removed & $\begin{array}{r}\% \text { leach } \\
\text { instial sollds }\end{array}$ \\
\hline $\mathrm{Ag}$ & & & 67.5 & 0.07 & & 0.000 & 0 & 0.000 & \\
\hline $\mathrm{Al}$ & $111000-155000$ & & 168000 & 177.41 & 1 & 1.774 & 83 & 145.477 & 82 \\
\hline As & & & & 0.00 & & 0.000 & t & 0,000 & \\
\hline $\mathrm{B}$ & $525 \cdot 2660$ & & 285 & 0.30 & 82 & 0.247 & 67 & 0.036 & 12 \\
\hline Ba & $547-1330$ & & 52 & 0.05 & 0 & 0.000 & 0 & 0.000 & 0 \\
\hline Ge & & & & ए.00 & & 0.000 & 0 & 0.000 & \\
\hline 日l & $5920-7400$ & & 13900 & 14.68 & 0 & 0.000 & 0 & 0.000 & a \\
\hline $\mathrm{Ca}$ & $740-5250$ & & 2850 & 3.01 & 0 & 0.000 & 0 & 0.000 & 0 \\
\hline $\mathrm{Cd}$ & & & 16 & 0.02 & & 0.000 & 0 & 0.000 & \\
\hline $\mathrm{Ce}$ & & & & 0.00 & & 0.000 & 0 & 0.000 & \\
\hline Co & $1040-1480$ & & 117 & 0.12 & & 0.000 & 0 & 0.000 & \\
\hline $\mathrm{Cr}$ & $155-962$ & & 612 & 0.65 & 68 & 0.439 & 16 & 0.032 & 5 \\
\hline $\mathrm{Cu}$ & 88 & & 288 & 0.30 & 0 & 0.000 & 0 & 0.000 & \\
\hline $\mathrm{Fe}$ & $5770-14800$ & & 10100 & 10.67 & 0 & 0.000 & 0 & 0.000 & 0 \\
\hline$k$ & & & 1210 & 1.28 & & 0.000 & 0 & 0.000 & \\
\hline La & & & & 0.00 & & 0.000 & 0 & 0.000 & \\
\hline Li & & & & 0.00 & & 0.000 & 0 & 0.000 & \\
\hline $\mathrm{Mg}$ & $15 \cdot 437$ & & 1360 & 1.44 & 0 & 0.000 & 0 & 0.000 & 0 \\
\hline $\mathrm{Mn}$ & $2000-3770$ & & 2360 & 2.49 & & 0.000 & 0 & 0.000 & 0 \\
\hline Mo & & & & 0.00 & & 0.000 & 0 & 0.000 & \\
\hline $\mathrm{Na}$ & $29400-370000$ & & 109000 & 115.11 & 69 & 79.423 & 0 & 0000 & \\
\hline$N_{1}$ & & & & 0.00 & & 0.000 & 0 & 0.000 & \\
\hline $\mathrm{Pb}$ & $670-730$ & & 491 & 0.52 & 0 & 0.000 & 0 & 0.000 & \\
\hline $\mathrm{Pd}$ & & & & 0.00 & & 0.000 & 0 & 0.000 & \\
\hline $\mathrm{Rh}$ & & & & 0.00 & & Q.,000 & 0 & 0.000 & \\
\hline Ru & & & & 0.00 & & 0.000 & 0 & 0.000 & \\
\hline Sb & & & & 0.00 & & 0.000 & 0 & 0.000 & \\
\hline Se & & & & 0.00 & & 0.000 & 0 & 0.000 & \\
\hline \$। & $5250-13300$ & & 10900 & 11.51 & 5 & 0.576 & 4 & 0.460 & 4 \\
\hline Sn & & & & 0.00 & & 0.000 & 0 & 0.000 & \\
\hline Sr & $252-481$ & & 402 & 0.42 & & 0.000 & 0 & 0.000 & 0 \\
\hline $\mathrm{Te}$ & & & & 0.00 & & 0.000 & 0 & 0.000 & \\
\hline Th & & & & 0.00 & & 0.000 & 0 & 0.000 & \\
\hline $\mathrm{Ti}$ & & & & 0.00 & & 0.000 & 0 & 0.000 & \\
\hline$U$ & $319-13300$ & & 8520 & 9.00 & 0 & 0.000 & 0 & 0.000 & 0 \\
\hline v & & & & 0.00 & & 0.000 & 0 & 0.000 & \\
\hline$W$ & & & & 0.00 & & 0.000 & 0 & 0.000 & \\
\hline$Y$ & & & & 0.00 & & 0.000 & 0 & 0.000 & \\
\hline $\mathrm{Zn}$ & $133-888$ & & 183 & 0.19 & 1 & 0.002 & 64 & 0.122 & 63 \\
\hline $\mathrm{Zr}$ & $104-660$ & & 219 & 0.23 & 0 & 0.000 & 0 & 0.000 & 0 \\
\hline
\end{tabular}

\begin{tabular}{|c|c|c|c|c|c|c|c|c|c|}
\hline \multirow[b]{2}{*}{ Component } & \multirow{2}{*}{$\begin{array}{r}\text { range } \\
\text { uglg sample } \\
\text { [Lumetta 1994] }\end{array}$} & \multirow[b]{2}{*}{$\begin{array}{r}\% \\
\text { recovery }\end{array}$} & \multirow[b]{2}{*}{$\begin{array}{l}\text { ug/g sample } \\
\text { (Colton 1995) }\end{array}$} & \multirow[b]{2}{*}{$\begin{array}{r}\text { MT } \\
\text { (tank) }\end{array}$} & \multicolumn{2}{|c|}{ Wash } & \multicolumn{3}{|c|}{ Leach } \\
\hline & & & & & $\begin{array}{l}\% \text { removed } \\
\text { initial solids }\end{array}$ & MT remaved & $\begin{array}{r}\% \text { removed } \\
\text { washed solids }\end{array}$ & MT removed & $\begin{array}{l}\% \text { removed } \\
\text { initial solids }\end{array}$ \\
\hline$\overline{\mathrm{OH}}$ & & & & 0.00 & & 0.000 & 0 & 0.000 & \\
\hline $\mathrm{NO3}-$ & & & 31700 & 33.48 & & 0.000 & 0 & 0.000 & \\
\hline $\mathrm{NO} 2$ & & & 6620 & 6.99 & & 0.000 & .0 & 0.000 & \\
\hline$P O 4.3$ & $15500-42200$ & & 43900 & 46.36 & 90 & 41.723 & 80 & 3709 & 8 \\
\hline $\mathrm{SOA}-2$ & & & 1290 & 1.36 & & 0.000 & 0 & 0.000 & \\
\hline F- & & & 7430 & 7.85 & & 0.000 & 0 & 0.000 & \\
\hline $\mathrm{Cl}$ & & & 975 & 1.03 & & 0.000 & 0 & 0.000 & \\
\hline $\mathrm{Br}-$ & & & & 0.00 & & 0.000 & 0 & 0000 & \\
\hline & $\begin{array}{r}\text { range } \\
\text { ucilg sample } \\
\text { [Lumetta 1994) }\end{array}$ & $\begin{array}{r}\% \\
\text { recovery }\end{array}$ & $\begin{array}{l}\text { ucilg sample } \\
\text { (Colton 1995) }\end{array}$ & $\begin{array}{r}\mathrm{Ci} \\
{[\tan k]}\end{array}$ & $\begin{array}{l}\% \text { romoved } \\
\text { initial solids }\end{array}$ & Ci removed & $\begin{array}{l}\text { \% removed } \\
\text { washed solids }\end{array}$ & Gi removed & $\begin{array}{l}\% \text { removed } \\
\text { inltial solidis }\end{array}$ \\
\hline Jotal alpha & & & & 0.00 & & 0.000 & 0 & 0.000 & \\
\hline Pu-239, 240 & & & 0.172 & 181.63 & & 0000 & 0 & 0.000 & \\
\hline$A m-241+P u-238$ & & & & 000 & & 0.000 & 0 & 0.000 & \\
\hline Am-241 (gamma) & & & 0.064 & 67.58 & & 0000 & 0 & 0.000 & \\
\hline $\mathrm{Cr}-243.244$ & & & & 000 & & 0.000 & 0 & 0.000 & \\
\hline Cs-137 & 139.386 & & 17.7 & 18691.47 & 7 & 1308.403 & 14 & 2429.891 & 13 \\
\hline $\mathrm{Co}-60$ & & & & 0.00 & & 0.000 & 0 & 0.000 & \\
\hline Eu-154 & & & & 000 & & 0.000 & 0 & 0.000 & \\
\hline Eu-155 & & & & 0.0 .0 & & 0.000 & 0 & 0.000 & \\
\hline Sr-90 & $222-411$ & & 261 & 275619.92 & 0 & 0000 & 1 & 2756.199 & 1 \\
\hline TC-99 & & & 0.005 & 5.28 & & 0.000 & 0 & 0.000 & \\
\hline
\end{tabular}

Cr concentration has been adjusted from reported value of 391 ugig in Colton 1995

Less than wash. leach values entered above as zero

Colton. N. G 1995. Sifdge Pretreatment Chemisty Evaluation: Enhanced Siudge Washtng Separation Factors. PNL-10512,

Pacific Northwest Natonal Laboratory. Richtand Washington.

Lumetra. G. J and B M Rapkb. 1994. Washing and Aikatine Leaching of Hantord Sludges: A Stalus Repport. PNL-10078,

Pacific Northwest National Laboratory. Richland Washingron. 


\section{Distribution}

No. of

Copies

OFFSITE

2 DOE/Office of Scientific and

Technical Information

Dennis Wynne

EM-361, Trevion II

U.S. Department of Energy

12800 Middlebrook Road

Germantown, MD 20874

John Grantham

Washington Department of Ecology

P.O. Box 47600

Olympia, WA $98594-7600$

Donald Temer

Los Alamos National Laboratory

P.O. Box 1663

Mail Stop G740

Los Alamos, NM 87545

Steven Agnew

Los Alamos National Laboratory

P.O. Box 1663

Mail Stop J586

Los Alamos, NM 87545

Ed Beahm

Oak Ridge National Laboratory

P.O. Box 2008

Oak Ridge, TN 37831-6221
No. of

Copies

Phil McGinnis

Oak Ridge National Laboratory

P.O. Box 2008

Oak Ridge, TN 37831-6273

Zane Egan

Oak Ridge National Laboratory

P.O. Box 2008

Oak Ridge, TN 37831-6223

Rodney Hunt

Oak Ridge National Laboratory

P.O. Box 2008

Oak Ridge, TN 37831

Major Thompson

Bldg. 773-A

Westinghouse Savannah River

Company

P. O. Box 616

Aiken, SC 29802

George Vandegrift

Argonne National Laboratory

9700 S. Cass Avenue

Argonne, IL 60439

John Swanson

1318 Cottonwood Dr.

Richland, WA 99352 